NATIONAL LABORATORY

\title{
Compatibility Study for Plastic, Elastomeric, and Metallic Fueling Infrastructure Materials Exposed to Aggressive Formulations of Isobutanol- Blended Gasoline
}

\section{August 2013}

Prepared by

Michael D. Kass

Christopher J. Janke

Steven J. Pawel

Jeffrey K. Thomson

Harry Meyer

Timothy J. Theiss

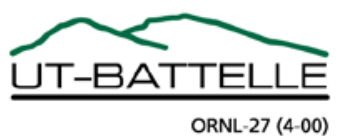




\section{DOCUMENT AVAILABILITY}

Reports produced after January 1, 1996, are generally available free via the U.S. Department of Energy (DOE) Information Bridge.

Web site http://www.osti.gov/bridge

Reports produced before January 1, 1996, may be purchased by members of the public from the following source.

National Technical Information Service

5285 Port Royal Road

Springfield, VA 22161

Telephone 703-605-6000 (1-800-553-6847)

TDD 703-487-4639

Fax 703-605-6900

E-mail info@ntis.gov

Web site http://www.ntis.gov/support/ordernowabout.htm

Reports are available to DOE employees, DOE contractors, Energy Technology Data Exchange (ETDE) representatives, and International Nuclear Information System (INIS) representatives from the following source.

Office of Scientific and Technical Information

P.O. Box 62

Oak Ridge, TN 37831

Telephone 865-576-8401

Fax 865-576-5728

E-mail reports@osti.gov

Web site http://www.osti.gov/contact.html

This report was prepared as an account of work sponsored by an agency of the United States Government. Neither the United States Government nor any agency thereof, nor any of their employees, makes any warranty, express or implied, or assumes any legal liability or responsibility for the accuracy, completeness, or usefulness of any information, apparatus, product, or process disclosed, or represents that its use would not infringe privately owned rights. Reference herein to any specific commercial product, process, or service by trade name, trademark, manufacturer, or otherwise, does not necessarily constitute or imply its endorsement, recommendation, or favoring by the United States Government or any agency thereof. The views and opinions of authors expressed herein do not necessarily state or reflect those of the United States Government or any agency thereof. 


\section{COMPATIBILITY STUDY FOR PLASTIC, ELASTOMERIC, AND METALLIC FUELING INFRASTRUCTURE MATERIALS EXPOSED TO AGGRESSIVE FORMULATIONS OF ISOBUTANOL-BLENDED GASOLINE}

Michael D. Kass, Christopher J. Janke, Steven J. Pawel, Jeffrey K. Thomson, Harry Meyer, and Timothy J. Theiss

Date Published: August 2013

Prepared by OAK RIDGE NATIONAL LABORATORY

Oak Ridge, Tennessee 37831-6283

managed by

UT-BATTELLE, LLC

for the

U.S. DEPARTMENT OF ENERGY

under contract DE-AC05-00OR22725 



\section{CONTENTS}

Page

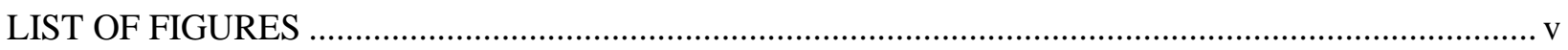

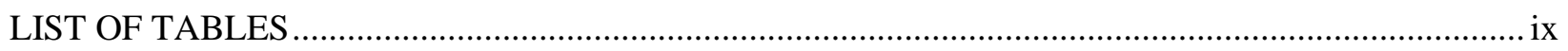

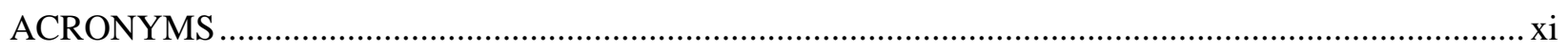

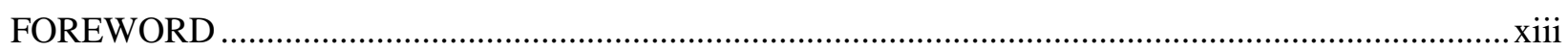

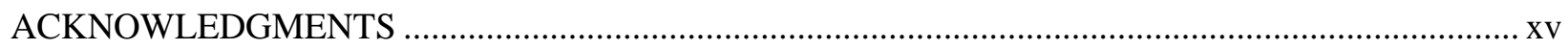

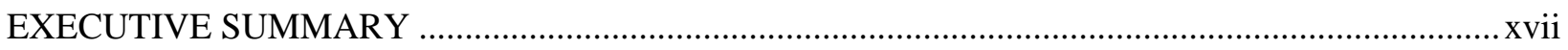

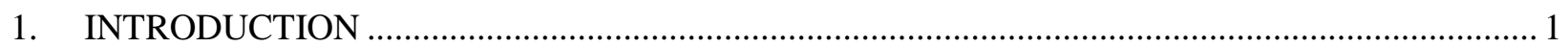

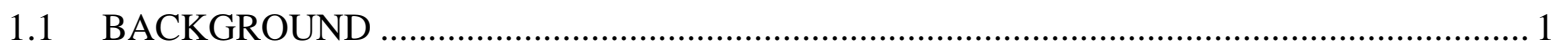

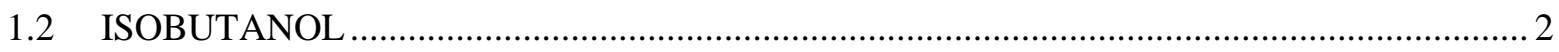

1.3 AGGRESSIVE ISOBUTANOL FORMULATION ......................................................... 2

1.4 PROPERTIES RELATIVE TO POLYMER-FUEL COMPATIBILITY ................................... 3

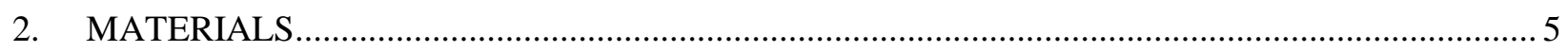

2.1 SELECTION AND DESCRIPTION OF ELASTOMERIC MATERIALS ............................. 5

2.2 SELECTION AND DESCRIPTION OF PLASTIC MATERIALS ........................................ 6

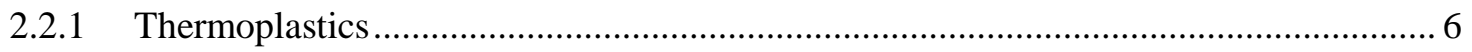

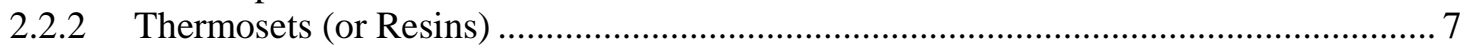

2.3 SELECTION AND DESCRIPTION OF METALLIC MATERIALS .................................. 8

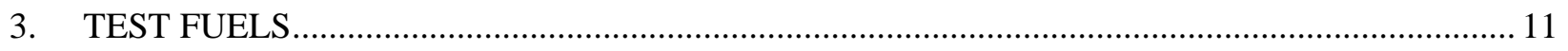

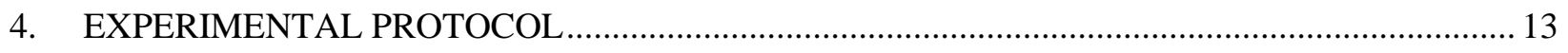

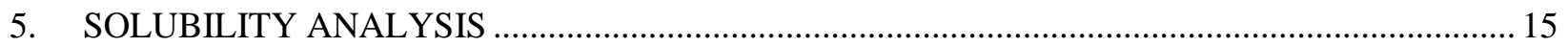

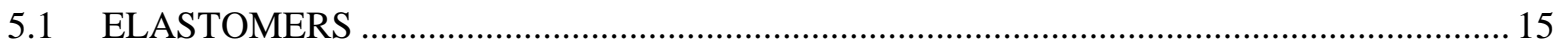

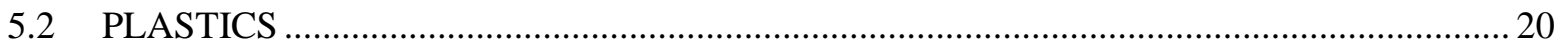

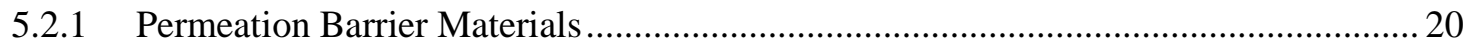

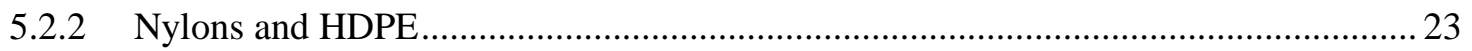

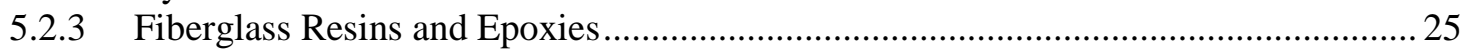

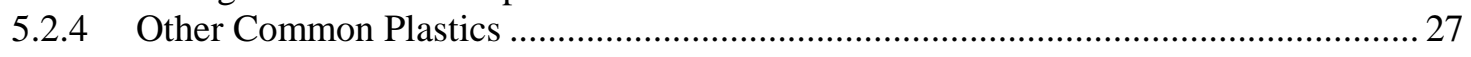

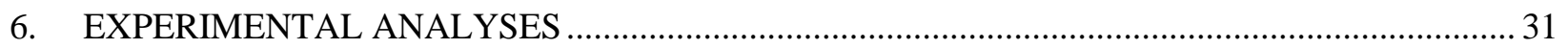

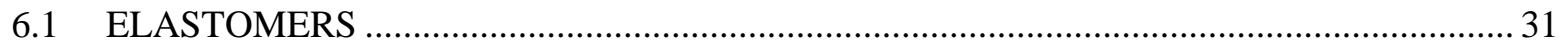

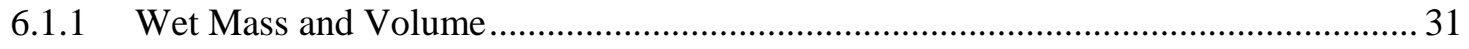

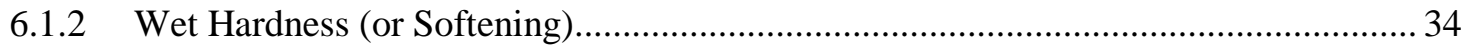

6.1.3 Dried mass and volume for wetted specimens............................................................ 37

6.1.4 Hardness Change Following Drying for Wetted and Vapor-Phase Specimens ............ 40

6.1.5 Dynamic Mechanical Analysis Results for Elastomers ............................................... 42

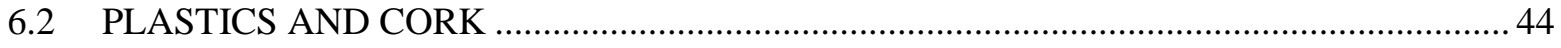

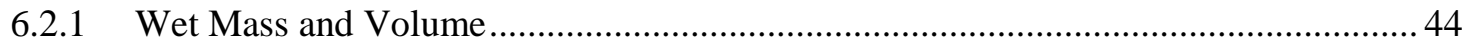

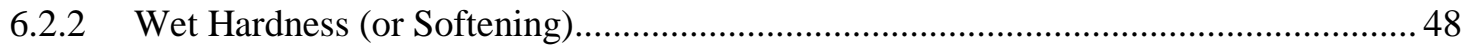

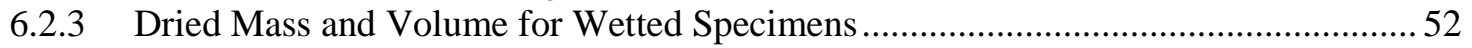

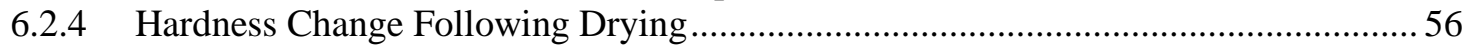

6.2.5 Dynamic Mechanical Analysis Results for Plastics.................................................... 59 


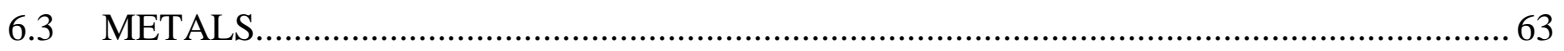

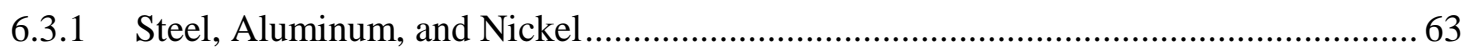

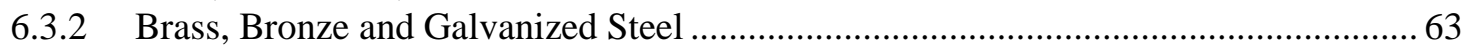

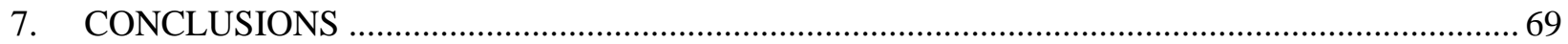

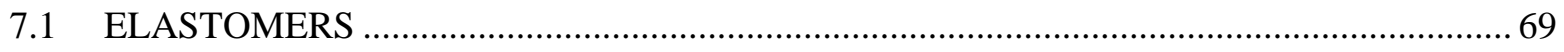

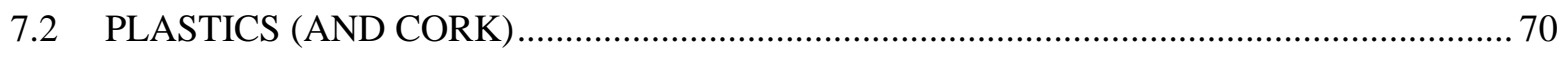

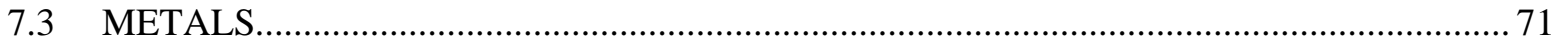

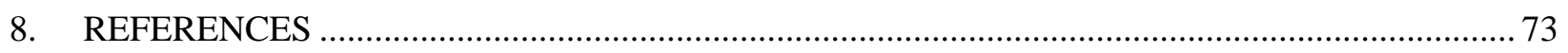

APPENDIX A. DMA RESULTS FOR ELASTOMERS ….............................................................. A-1

APPENDIX B. DMA RESULTS FOR PLASTICS AND CORK MATERIALS .................................... B-1 


\section{LIST OF FIGURES}

Figure $\quad$ Page

1. Molecular diagrams of ethanol and isobutanol......................................................................... 2

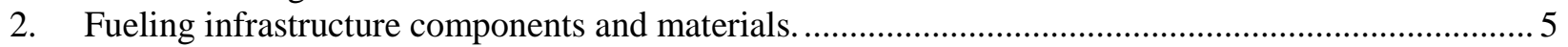

3. Appearance of galvanically coupled specimens showing exposed surfaces...................................... 9

4. Mounted metal specimens and mounting hardware. Each fixture held duplicate metallic specimens isolated from each other and the stainless steel hardware by Teflon components.............. 9

5. Arrangement and components of dynamic environmental chamber. .............................................. 13

6. Plan view of the interior of the environmental chamber showing arrangement of specimens and the liner. Specimens are shown attached to the liner. .............................................................. 14

7. Flowchart outlining test and measurement protocols for each material type.................................... 14

8. Solubility distance curves for fluoroelastomers for each alcohol type and concentration. The

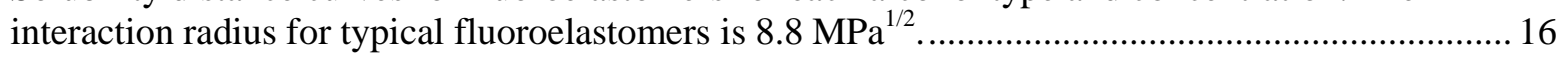

9. Solubility distance curves for NBR for each alcohol type and concentration. The interaction

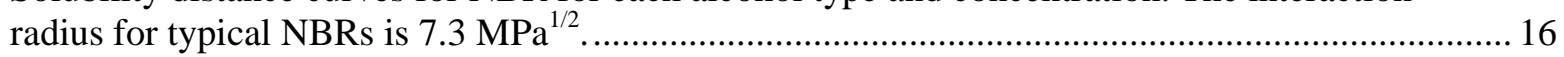

10. Solubility distance curves for SBR for each alcohol type and concentration. The interaction radius for SBR is $7.3 \mathrm{MPa}^{1 / 2}$

11. Solubility distance curves for neoprene for each alcohol type and concentration. The interaction radius for neoprene is $8.9 \mathrm{MPa}^{1 / 2}$.

12. Solubility distance curves for polyurethane for each alcohol type and concentration. The interaction radius for polyurethane is $8.0 \mathrm{MPa}^{1 / 2}$

13. Solubility distance curves for silicone rubber for each alcohol type and concentration. The interaction radius for silicone rubber is $14.3 \mathrm{MPa}^{1 / 2}$....

14. Solubility distance curves for PPS for each alcohol type and concentration. The interaction radius for PPS is $2.8 \mathrm{MPa}^{1 / 2}$.

15. Solubility distance curves for PTFE for each alcohol type and concentration. The interaction radius for PTFE is $3.9 \mathrm{MPa}^{1 / 2}$.

16. Solubility distance curves for PVDF for each alcohol type and concentration. The interaction radius for PVDF is $4.1 \mathrm{MPa}^{1 / 2}$.

17. Solubility distance curves for PET for each alcohol type and concentration. The interaction radius for PET is $5.0 \mathrm{MPa}^{1 / 2}$.

18. Solubility distance curves for Nylon 6 for each alcohol type and concentration. The interaction radius for Nylon 6 is $5.1 \mathrm{MPa}^{1 / 2}$.

19. Solubility distance curves for Nylon 6,6 for each alcohol type and concentration. The interaction radius for Nylon 6,6 is $4.4 \mathrm{MPa}^{1 / 2}$.

20. Solubility distance curves for Nylon 12 for each alcohol type and concentration. The interaction radius for Nylon 12 is $6.3 \mathrm{MPa}^{1 / 2}$.

21. Solubility distance curves for Nylon 11 for each alcohol type and concentration. The interaction radius for nylon 11 is $5.1 \mathrm{MPa}^{1 / 2}$.

22. Solubility distance curves for HDPE for each alcohol type and concentration. The interaction radius for HDPE is $2.0 \mathrm{MPa}^{1 / 2}$.

23. Solubility distance curves for terephthalic and isophthalic polyester resins for each alcohol type and concentration. The interaction radius for these resins is $18.0 \mathrm{MPa}^{1 / 2}$.

24. Solubility distance curves for vinyl ester resins for each alcohol type and concentration. The interaction radius for vinyl ester is $15.1 \mathrm{MPa}^{1 / 2}$

25. Solubility distance curves for epoxy resins for each alcohol type and concentration. Two curing processes (hot and cold) are included for comparison. The interaction radii for cold and hot cures are 8.8 and $9.7 \mathrm{MPa}^{1 / 2}$, respectively. 
26. Solubility distance curves for PET and PETG for each alcohol type and concentration. The interaction radius for PETG is $6.0 \mathrm{MPa}^{1 / 2}$.

27. Solubility distance curves for PBT for each alcohol type and concentration. The interaction radius for $\mathrm{PBT}$ is $4.5 \mathrm{MPa}^{1 / 2}$.

28. Solubility distance curves for POM for each alcohol type and concentration. The interaction radius for POM is $5.3 \mathrm{MPa}^{1 / 2}$.

29. Solubility distance curves for PP for each alcohol type and concentration. The interaction radius for $\mathrm{PP}$ is $6.0 \mathrm{MPa}^{1 / 2}$.

30. Mass and volume increases for the elastomers exposed to the test fuel liquids for 4 weeks.............. 31

31. Mass and volume increases for the fluoroelastomers exposed to the test fuel liquids for 4 weeks.

32. Mass and volume increases for the NBRs exposed to the test fuel liquids for 4 weeks.

33. Mass and volume increases for the polyurethane, neoprene, SBR, and silicone specimens exposed to the test fuel liquids for 4 weeks.

34. Hardness decrease (softening) of elastomers exposed to the liquid test fuels. Specimens were tested while wet.

35. Hardness decrease (softening) of the fluoroelastomer specimens exposed to the liquid test fuels. Specimens were tested while wet.

36. Hardness decrease (softening) of the six NBR specimens exposed to the liquid test fuels................ 36

37. Hardness decrease (softening) of polyurethane, neoprene, SBR, and silicone rubber specimens exposed to the liquid test fuels.

38. Volume change of fluoroelastomers exposed to the liquid test fuels after drying at $60^{\circ} \mathrm{C}$ for 20 hours.

39. Volume change of NBRs exposed to the liquid test fuels after drying at $60^{\circ} \mathrm{C}$ for 20 hours.

40. Volume change of polyurethane, neoprene, SBR, and silicone rubber specimens exposed to the liquid test fuels after drying at $60^{\circ} \mathrm{C}$ for 20 hours.

41. Point change in hardness (after drying) from the baseline condition for fluoroelastomers in the liquid and vapor phases of the test fuel liquids.

42. Point change in hardness (after drying) from the baseline condition for NBRs in the liquid and vapor phases of the test fuel liquids

43. Point change in hardness (after drying) from the baseline condition for polyurethane, neoprene, SBR, and silicone in the liquid and vapor phases of the test fuels.

44. Glass transition temperature results for fluoroelastomers exposed to the test fuel liquid and vapor regions.

45. Glass transition temperature results for NBRs exposed the test fuel liquid and vapor regions.......... 43

46. Glass transition temperature results for polyurethane, neoprene, SBR, and silicone rubbers exposed the test fuel liquid and vapor regions.

47. Mass and volume change for plastic materials typically used as permeation barriers.

Specimens were immersed in the test fuel liquids for 16 weeks at $60^{\circ} \mathrm{C}$.

48. Mass and volume change for nylons and HDPE. Specimens were immersed in the test fuel liquids for 16 weeks at $60^{\circ} \mathrm{C}$.

49. Mass and volume change for two resin materials. Specimens were immersed in the test fuel liquids for 16 weeks at $60^{\circ} \mathrm{C}$.

50. Mass and volume change for other common plastic materials. Specimens were immersed in the test fuel liquids for 16 weeks at $60^{\circ} \mathrm{C}$.

51. Mass and volume change for rubberized cork materials. Specimens were immersed in the test fuel liquids for 16 weeks at $60^{\circ} \mathrm{C}$.

52. Point change in hardness while in the wetted state for plastic materials typically used as permeation barriers. Specimens were immersed in the test fuel liquids for 16 weeks at $60^{\circ} \mathrm{C}$

53. Point change in hardness while in the wetted state for nylons and HDPE. Specimens were immersed in the test fuel liquids for 16 weeks at $60^{\circ} \mathrm{C}$. 
54. Point change in hardness while in the wetted state for two fiberglass resin materials.

Specimens were immersed in the test fuel liquids for 16 weeks at $60^{\circ} \mathrm{C}$.

55. Point change in hardness while in the wetted state for other common plastic materials.

Specimens were immersed in the test fuel liquids for 16 weeks at $60^{\circ} \mathrm{C}$.

56. Point change in hardness while in the wetted state for cork materials. Specimens were immersed in the test fuel liquids for 16 weeks at $60^{\circ} \mathrm{C}$.

57. Dried mass and volume change for permeation barrier plastics. Specimens were immersed in the test fuel liquids for 16 weeks at $60^{\circ} \mathrm{C}$ and then dried at $60^{\circ} \mathrm{C}$ for 65 hours.

58. Dried mass and volume change for nylons and HDPE. Specimens were immersed in the test fuel liquids for 16 weeks at $60^{\circ} \mathrm{C}$ and then dried at $60^{\circ} \mathrm{C}$ for 65 hours.

59. Dried mass and volume change for two fiberglass resin materials. Specimens were immersed in the test fuel liquids for 16 weeks at $60^{\circ} \mathrm{C}$ and then dried at $60^{\circ} \mathrm{C}$ for 65 hours.

60. Dried mass and volume change for other common plastic materials. Specimens were immersed in the test fuel liquids for 16 weeks at $60^{\circ} \mathrm{C}$ and then dried at $60^{\circ} \mathrm{C}$ for 65 hours............................ 55

61. Dried mass and volume change or two rubber-impregnated cork materials. Specimens were immersed in the test fuel liquids for 16 weeks at $60^{\circ} \mathrm{C}$ and then dried at $60^{\circ} \mathrm{C}$ for 65 hours............ 55

62. Point change in hardness (after drying) for plastic materials typically used as permeation barriers.

63. Point change in hardness (after drying) for the nylon materials and HDPE.................................... 56

64. Point change in hardness (after drying) for the resin materials. .................................................. 57

65. Point change in hardness (after drying) for other common plastic materials. ................................. 57

66. Point change in hardness (after drying) for the cork materials. ..................................................59

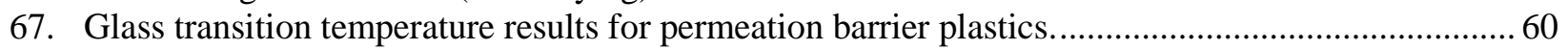

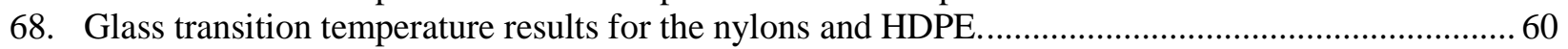

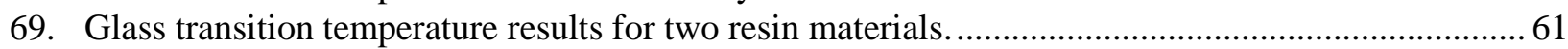

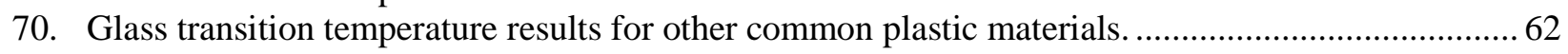

71. Glass transition temperature results for the rubberized cork materials........................................... 62

72. Unexposed and post-exposure appearance of the cartridge brass, phosphor bronze, and galvanized steel specimens following 4 weeks of immersion in CiBu16a and CiBu24a at $60^{\circ} \mathrm{C}$...... 64

73. Post-exposure appearance of other metallic specimens included in the study following 4 weeks of exposure to immersion in $\mathrm{CiBu} 16 \mathrm{a}$ at $60^{\circ} \mathrm{C}$.

74. Post-exposure appearance of other coated and galvanic coupled specimens included in the study following 4 weeks of exposure to immersion in CiBu16a at $60^{\circ} \mathrm{C}$.

75. XPS depth profile for phosphor bronze on an area of the surface protected by a Teflon washer following immersion in CiBu16a for 4 weeks at $60^{\circ} \mathrm{C}$.

76. XPS depth profile for phosphor bronze on an exposed area of the surface following immersion in CiBu16a for 4 weeks at $60^{\circ} \mathrm{C}$.

77. XPS depth profile for cartridge brass on an area of the surface protected by a Teflon washer following immersion in CiBu16a for 4 weeks at $60^{\circ} \mathrm{C}$.

78. XPS depth profile for cartridge brass on an exposed area of the surface following immersion in CiBu16a for 4 weeks at $60^{\circ} \mathrm{C}$. 



\section{LIST OF TABLES}

\section{Table}

Page

1. Formulation used to make $1 \mathrm{~L}$ of aggressive isobutanol ................................................................. 3

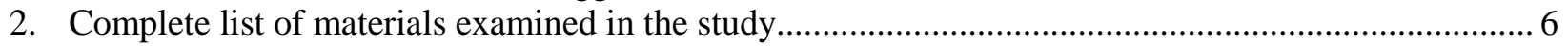

3. Categories and types of plastic materials examined in the study ..................................................... 7

4. List of single and plated metallic materials examined in the study ..................................................... 8

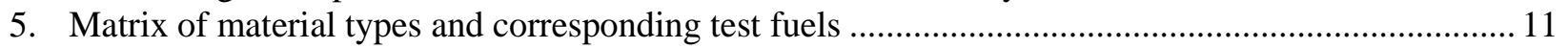

6. Volume swell (or solubility) relationship with interaction radius and solubility distance..................15

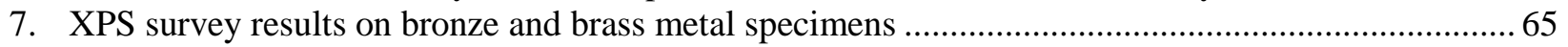





\section{ACRONYMS}

\begin{tabular}{|c|c|}
\hline API & American Petroleum Institute \\
\hline ASTM & American Society for Testing and Materials \\
\hline BGPY & billion gallons per year \\
\hline CEXXa & $\begin{array}{l}\text { Test fuel containing XX amount of aggressive ethanol with the balance composed of } \\
\text { Fuel C }\end{array}$ \\
\hline $\mathrm{CiBuXXa}$ & $\begin{array}{l}\text { Test fuel containing XX amount of aggressive isobutanol with the balance composed of } \\
\text { Fuel C }\end{array}$ \\
\hline CRC & Coordinating Research Council \\
\hline DMA & dynamic mechanical analysis \\
\hline DOE & U.S. Department of Energy \\
\hline$d_{s}$ & solubility distance \\
\hline E25 & gasoline containing $25 \%$ ethanol by volume \\
\hline E85 & gasoline containing $85 \%$ ethanol by volume \\
\hline ECO & epichlorohydrin \\
\hline EERE & Office of Energy Efficiency and Renewable Energy (of DOE) \\
\hline EISA & Energy Independence and Security Act \\
\hline EPA & U.S. Environmental Protection Agency \\
\hline EPA-OUST & EPA Office of Underground Storage Tanks \\
\hline FFV & flex-fuel vehicle \\
\hline FRP & fiber-reinforced plastic \\
\hline Fuel C & gasoline test fuel composed of $50 \mathrm{vol} \%$ toluene and $50 \mathrm{vol} \%$ isooctane \\
\hline HDPE & high density polyethylene \\
\hline HSP & Hansen solubility parameter \\
\hline IR & interaction radius \\
\hline NBR & acrylonitrile (or nitrile) butadiene rubber \\
\hline NREL & National Renewable Energy Laboratory \\
\hline OBP & Office of Biomass Program (of DOE) \\
\hline ORNL & Oak Ridge National Laboratory \\
\hline PBT & polybutylene terephthalate \\
\hline PEI & Petroleum Equipment Institute \\
\hline PET & polyethylene terephthalate \\
\hline PETG & polyethylene terephthalate co-polymer \\
\hline POM & polyoxymethylene \\
\hline PP & polypropylene \\
\hline PPS & polyphenylene sulfide \\
\hline PTFE & polytetrafluoroethylene \\
\hline PTU & polythiourea \\
\hline PVDF & polyvinylidene fluoride \\
\hline RFS & Renewable Fuel Standard \\
\hline RT & room temperature \\
\hline SAE & Society of Automotive Engineers \\
\hline SBR & styrene-butadiene rubber \\
\hline $\mathrm{T}_{\mathrm{g}}$ & glass-to-rubber transition temperature \\
\hline UL & Underwriters Laboratories \\
\hline VTP & Vehicle Technologies Program (of DOE) \\
\hline XPS & x-ray photoelectron spectroscopy \\
\hline
\end{tabular}





\section{FOREWORD}

The results obtained in this study build upon earlier efforts focused on assessment of fueling infrastructure materials with ethanol-blended gasoline. It is not the purpose of this study to define the acceptable limits of material performance or to rate individual materials. Rather, the goal was to measure critical property changes (volume, hardness, mass, etc.) for representative classes of fueling storage and dispenser materials in test fluids representing blends of gasoline and isobutanol. The test results were compared against CE10a and CE17a (for elastomers and metals) and CE25a for plastics to examine the relative compatibility of infrastructure materials with fuel blends containing similar concentration of ethanol or isobutanol. It is anticipated that these results will be used by material designers and users to guide the selection and development of materials compatible for use in ethanol- and isobutanol-blended gasoline dispensers, up to and including $24 \%$ isobutanol. 



\section{ACKNOWLEDGMENTS}

This report and the work described were sponsored by Butamax Advanced Biofuels, LLC, and the authors gratefully acknowledge the support received from James Baustian, Fuel Product Manager for Butamax. Important insights were also provided to the ORNL research team by James Baustian and Les Wolf (also of Butamax, LLC) and Wolf Koch of Technology Resources International, Inc. This work also benefitted from discussions with the corresponding Underwriters Laboratories research team, which included Tom Chapin, Ken Boyce, and Edwin Yang. The manuscript was greatly improved by the review and input of Brian West and Scott Sluder from ORNL. 



\section{EXECUTIVE SUMMARY}

\section{Background}

The Energy Independence and Security Act (EISA) of 2007 is an omnibus energy policy law designed to move the United States toward greater energy security and independence. A key provision of EISA is the Renewable Fuel Standard (RFS), which requires the nation to use 36 billion gallons per year (BGPY) of renewable fuel in vehicles by 2022. ${ }^{*}$ Ethanol is the most widely used renewable fuel, and increasing the allowable ethanol content from $10 \%$ to $15 \%$ is expected to push renewable fuel consumption to as much as 21 BGPY. Therefore, a large portion of the 36 billion gallon requirement can be met by increasing the ethanol content in gasoline to $15 \%$. However, raising the ethanol content to $15 \%$, by itself, will still not fully meet the RSF requirement, and concerns have been raised that this increase in ethanol may not be entirely compatible with current and legacy materials used in standard gasoline fueling systems. In the summer of 2008, the U.S. Department of Energy (DOE) recognized the need to assess the impact of intermediate blends of ethanol on the fueling infrastructure, specifically those systems located at the fueling station. A short time later (March 2009), Growth Energy (a coalition of ethanol producers and supporters) requested a waiver from the U.S. Environmental Protection Agency (EPA) to allow the use of $15 \%$ ethanol in gasoline. ${ }^{\dagger}$

In 1981 the EPA approved a waiver (known as the OCTAMIX waiver) that allowed other alcohol types (such as methanol) to be blended with gasoline as long as the resulting fuel blend was substantially similar to gasoline. ${ }^{\ddagger}$ Another requirement is that the fuel oxygen content cannot exceed $3.7 \mathrm{wt} \%$. Currently, this requirement limits ethanol in gasoline to $10 \mathrm{vol} \%$. The corresponding oxygen equivalent for isobutanol is $16 \mathrm{vol} \%$; therefore, isobutanol offers a pathway towards meeting the RFS requirement by allowing greater biofuel usage than can be achieved by ethanol alone. In fact, the United States has already hit the "blend wall" for ethanol, the point at which virtually all gasoline sold is E10 and no remaining market is left for ethanol, meaning that the country cannot substantially increase ethanol usage unless the EPA allows for higher levels of ethanol or until E85 use is expanded significantly.

Butamax Advanced Biofuels, LLC has developed proprietary technologies to convert corn into isobutanol. ${ }^{\S}$ Much of the infrastructure already existing at ethanol plants can be readily converted to produce isobutanol instead. The focus of this investigation was to compare the compatibility of fueling infrastructure materials (such as elastomers, plastics, and metals) to test fuels representing E10 and E15 and their corresponding oxygen equivalents with isobutanol (iBu16 and iBu24). Butamax sponsored a research project at Oak Ridge National Laboratory (ORNL) to perform empirical studies using aggressive fuel formulations representing 16 and $24 \%$ isobutanol. The results contained within this report include a solubility analysis for each material type and fuel formulation and empirical results for elastomers, plastics, and metals. Data obtained from the preceding ethanol compatibility studies on these materials are included for additional interpretation and summary. ${ }^{*}+$

\footnotetext{
* Energy Independence and Security Act of 2007: A Summary of Major Provisions, CRS Report for Congress, Order Code RL34294, Dec. 21, 2007.

${ }^{\dagger}$ Partial Grant of Clean Air Act Waiver Application Submitted by Growth Energy to Increase Allowable Ethanol Content of Gasoline to 15 Percent; Decision of the Administrator (EPA-HQ-OAR-2009-0211; FRL-9258-6) Federal Register 76(17), 4662 (Jan. 26, 2011). USEPA website: www.epa.gov/otaq/regs/fuel/additive/e15.\#wn.

${ }^{\ddagger}$ https://www.federalregister.gov/articles/2012/06/07/2012-13823/regulation-of-fuel-and-fuel-additivesmodification-to-octamix-waiver-txceed.

$\S$ Butamax Advanced Biofuels website, http://www.butamax.com.

${ }^{* *}$ M. D. Kass, T. J. Theiss, C. J. Janke, S. J. Pawel, and S. A. Lewis, Intermediate Ethanol Blends Infrastructure Materials Compatibility Study: Elastomers, Metals, and Sealants, report ORNL/TM-2010/326, Oak Ridge National Laboratory, March 2011.
} 


\section{Experimental Overview}

The materials chosen for evaluation were similar to those selected in an earlier investigation that focused on the compatibility of ethanol-gasoline test fuels with the fueling infrastructure. Those experiments were supported by the DOE to assess fueling infrastructure material compatibility to E15 and E20. ${ }^{* *-\dagger}$ The material list was extensive and included sealants, elastomers, plastics, and metals. However, for the different classes of polymeric materials, specific grades and formulations were not precisely identified due to proprietary formulations and the sheer variety of grades for each polymer class. As a result, the material selection may not include specific grades or formulations used in legacy and the current typical gasoline fueling infrastructure.

In this study, two test fuels representing gasoline blended with 16 and 24\% isobutanol were prepared and used to assess material compatibility. The results from these two fuel formulations were compared to those from earlier studies investigating ethanol blends of Fuel C, CE10a, CE17a, and CE25a. (E10 and E17 have roughly the same oxygen content as 16 and 24\% isobutanol.) In these earlier studies, plastic materials were not included in the CE10a and CE17a test matrices, but they were included in the CE25a study. The test fuels were based on the Fuel $\mathrm{C}$ composition and aggressive ethanol formulation described in SAE J1681, "Gasoline, Alcohol, and Diesel Fuel Surrogates for Materials Testing." "The " $C$ " represents the base fuel type, Fuel $\mathrm{C}$, which is a 50-50 mixture of toluene and isooctane and is representative of highly aromatic gasoline ( $>40 \%$ aromatics by volume). The other test fuels contain an aggressive ethanol solution added to Fuel C. The numbers that follow CE refer to the volume fraction of ethanol added to Fuel C, and the use of aggressive ethanol (containing water and trace levels of $\mathrm{NaCl}$, acetic and sulfuric acids) is represented by the "a". These contaminants are commonly found in ethanolgasoline fuels in the field and represent a reasonable upper limit for fuel-grade ethanol.

Currently, there is no standard for an aggressive isobutanol formulation, so one was developed on the basis of the impurities cited for ethanol (in SAE J1681) and the molar ratio of these impurities produced relative to the ethanol balance. Isobutyric acid was substituted for acetic acid in formulating aggressive isobutanol, and the molar ratio for isobutyric acid relative to isobutanol was identical to the ratio between acetic acid and ethanol in SAE J1681.

The metals and alloys that were evaluated in this study included single metal/alloy coupons of 1020 carbon steel, 304 stainless steel, 1100 aluminum, nickel 201, cartridge brass, phosphor bronze, and galvanized and terne-plated steels. To demonstrate the effect of joining of dissimilar metals in a galvanic couple (and/or flaws in protective platings), specimens composed of steel, brass, and aluminum were coupled with lead, zinc, chromium, and nickel. Corrosion was primarily assessed by mass loss and discoloration.

The elastomer materials included fluorocarbons, nitrile butadiene rubbers (NBRs), fluorosilicone, silicone, styrene butadiene rubber (SBR), polyurethane, and neoprene. Plastic specimens included representative thermoplastic and fiberglass resin polymers. These materials were grouped according to application. Those used as permeation barriers included polyphenylene sulfide (PPS), polyethylene terephthalate (PET), polytetrafluoroethylene (PTFE), and polyvinylidene fluoride (PVDF). PET and

\footnotetext{
${ }^{*}$ M. D. Kass, T. J. Theiss, C. J. Janke, and S. J. Pawel, Compatibility Study for Plastic, Elastomeric, and Metallic Fueling Infrastructure Materials Exposed to Aggressive Formulations of Ethanol-Blended Gasoline, report ORNL/TM-2012/88, Oak Ridge National Laboratory, May 2012.

${ }^{\dagger}$ S. J. Pawel, M. D. Kass, and C. J. Janke, Preliminary Compatibility Assessment of Metallic Dispenser Materials for Service in Ethanol Fuel Blends, report ORNL/TM-2009/086, Oak Ridge National Laboratory, November 2009.

‡ Society of Automotive Engineers, “Gasoline, Alcohol, and Diesel Fuel Surrogates for Materials Testing,” SAE J1681, issued September 1993, revised January 2000-01.
} 
PTFE are also known commercially as Mylar and Teflon, respectively. Structural plastics commonly used in flexible piping included four types of nylon and high density polyethylene (HDPE). Four fiberglass resins and four epoxies were included along with two rubberized cork materials.

The specimens were placed inside sealed environmental chambers capable of maintaining controlled temperature and dynamic fluid flow. Three specimens for each polymer type (and two for each metal type) were completely submerged in the test fuels during the exposure period. To reflect the conditions associated with vapor recovery, single specimens of each material were placed in the headspace region above the liquid fuel line to expose the samples to fuel vapors only. During the exposure period, the test fuel liquids were continuously stirred to maintain a flow rate of $0.8 \mathrm{~m} / \mathrm{s}$ at the specimen surfaces, and the temperature was held constant at $60^{\circ} \mathrm{C}$. Elastomer and metal specimens were exposed to Fuel C, CE10a, CE17a, CE15a, CiBu16a, and CiBu24a test fuel formulations, while the plastic and cork/NBR specimens were exposed to Fuel C, CE25a, CiBu16a, and CiBu24a. Cork impregnated with epichlorohydrin (ECO) rubber was added later to the material selection and, as a result, was only evaluated in CiBu16a and CiBu24a test fuels. After 4 weeks, the elastomers and metals were removed for property measurement, while the plastics and corks were exposed for 16 weeks to ensure that the test fuel had enough time to completely permeate the plastic specimens. The use of aggressive ethanol and isobutanol, along with elevated test temperature, is intended to minimize the length of exposure necessary to rigorously evaluate materials and also to follow a standard method of testing these fuel system materials.

The changes in mass, volume, and hardness (from the original baseline condition) were determined for each polymer specimen immersed in the test fuels. These measurements were made immediately upon removal of the specimens from the test fuel liquid (wetted state) and, again, after drying for 65 hours at $60^{\circ} \mathrm{C}$ (dried state). The headspace specimens were measured only for hardness. Dynamic mechanical analysis (DMA) was performed on each specimen to determine whether significant structural changes had occurred in the polymers. The temperature associated with the onset of the glass-to-rubber transitions was determined from the DMA measurement and reported for each material.

\section{Elastomer Results}

Some level of swelling (or volume expansion) upon exposure to fuels is expected for elastomeric materials, and this swelling serves to provide a tight seal to prevent leakage. The amount of acceptable volume expansion is highly dependent on the application of the seal, and in fact, volume swelling up to $50 \%$ is allowable in many cases. Excessive swelling, however, may cause the seal to extrude beyond the joined interfaces. Protruded seals (or gaskets) are subject to breakage and abrasion. High swelling corresponds to high fuel permeability, and the potential for polymer component extraction and vapor release (of the fuel through the polymer) exists.

For all elastomer materials evaluated in this study, the gasoline test fuel, Fuel C, was primarily responsible for the observed swelling and softening. The addition of aggressive alcohol to the base fuel, whether as isobutanol or ethanol, produced additional swelling for most materials. The extent of additional swelling produced with the isobutanol test fuels, CiBu16a and CiBu24a, was either similar to, or noticeably less than, that produced with oxygen-equivalent levels of ethanol (i.e., CE10a and CE17a). For SBR and silicone rubbers, $16 \%$ aggressive isobutanol showed no change in swell from the Fuel C baseline. However, 24\% aggressive isobutanol did produce significantly lower swelling than Fuel C.

The observed swelling was accompanied by a hardness decrease (or softening) from the original condition. However, the extent of softening was found to be more sensitive to fuel chemistry than the degree of observed swelling. For the fluoroelastomers, the level of softening corresponded to the increase in volume, and ethanol was found to produce the highest levels of both for these elastomers. The NBR specimens exhibited uniform swelling with all test fuels, but the alcohol additions, especially ethanol, 
produced significant additional softening. The alcohol additions also produced slight increases in softening for neoprene, SBR, and silicone. Polyurethane was unique in that it was the only elastomer that, when wetted, was not appreciably softened by Fuel C. However, when aggressive ethanol is added, the hardness decreases dramatically. Isobutanol also caused polyurethane to soften, but not nearly as much as was observed with ethanol.

The exposed samples were dried at $60^{\circ} \mathrm{C}$ for 20 hours to determine if the test fuels were effective at dissolution and extraction of one or more elastomer components, especially plasticizer additions. Plasticizers are typically phthalate chemicals, which are added to certain rubbers to improve pliability. The increased pliability improves the compression properties of the elastomer to provide a better seal. Removal of the plasticizers will cause the rubber to become brittle and potentially crack under compression. This embrittlement is accompanied by an increase in hardness from the baseline value.

After drying, both silicone and fluorosilicone returned to their original volume and hardness levels, indicating that neither of these elastomer types was structurally affected by the test fuels. The fluorocarbons showed a small volume increase and drop in hardness after drying that was attributed to the retention of the test fuel in their microstructure. The NBR samples all exhibited a high level of shrinkage (10-18\%) and embrittlement. In all cases Fuel C was the fuel component primarily responsible for shrinkage, although ethanol did produce a small additional contribution. Isobutanol, on the other hand, was observed to have no added effect on NBR shrinkage. Interestingly, embrittlement was actually slightly reduced for those test fuels containing ethanol and isobutanol, with isobutanol producing the lowest levels of embrittlement for the NBRs. Neoprene and SBR exhibited considerable shrinkage with exposure, but virtually all of this effect was caused by Fuel C. Fuel C also appears to be the primary factor causing neoprene embrittlement, whereas SBR was unaffected, except for a slight embrittlement when exposed to the test fuels containing isobutanol. The addition of aggressive ethanol to the base fuel did not produce any additional shrinkage or embrittlement in either neoprene or SBR. Dry-out properties were also determined for specimens exposed to the test fuel vapors, and it was shown that the results closely paralleled those of the wetted specimens.

Analysis of the DMA test results showed that glass transition temperature was unaffected by the test fuels for fluorosilicone, polyurethane, neoprene, SBR, and silicone. The fluorocarbons showed a slight drop $\left(10^{\circ} \mathrm{C}\right)$ in the transition temperature following exposure to the test fuels, whereas the NBRs exhibited around a $20^{\circ} \mathrm{C}$ increase in transition temperature. In these cases, Fuel C was the component primarily responsible for the shift in the transition temperature from baseline.

\section{Plastic Results}

A predictive model was constructed using Hansen solubility theory for each material. ${ }^{*}$ The parameters used in this model were derived from literature sources that may or may not exactly reflect the specific material types and compositions used. In spite of this limitation, the predicted results calculated by the Hansen solubility method generally provided good to excellent agreement to the observed volume swell for many of the plastic materials. (PVDF and Nylon 11 were notable exceptions.) The plastics that exhibited the lowest volume swelling (and smallest change in hardness) in the test fuels were the permeation barrier materials: PPS, PET, PVDF, and PTFE. These four materials exhibited negligible swelling with Fuel C and the isobutanol test fuels. However, PVDF did undergo a modest $5 \%$ volume expansion with exposure to CE25a. The three petroleum-based nylons behaved similarly to PVDF; they also showed negligible swell with Fuel $\mathrm{C}$ and the isobutanol test fuel, but swelled to around 5\% in CE25a. Nylon 11, which is made from vegetable oil, expanded 5\% in Fuel C, 18\% in CE25a, and around 15\% in

\footnotetext{
* C. M. Hansen, Hansen Solubility Parameters: A User’s Handbook, 2nd ed., CRC Press, Boca Raton, Florida, 2007.
} 
the test fuels containing isobutanol. The added ethanol also caused significant softening in Nylon 11 relative to the other test fuels. HDPE underwent a modest and consistent amount of swell following exposure to the test fuels; the added alcohols did not appear to have an effect.

Four fiberglass resins were tested and all were found to be sensitive to alcohol blends. Only two of the four resins (terephthalic polyester and vinyl ester) remained intact following exposure to CE25a, $\mathrm{CiBu16a}$, and CiBu24a. (The two isophthalic polyesters and the epoxy resins fractured during the exposure runs and are not viewed as compatible with gasoline-alcohol fuels.) Terephthalic polyester swelled around 7\% with Fuel C, but swelling increased to around 25\% for the fuels containing either ethanol or isobutanol. The vinyl ester resin performed much better, as it exhibited modest swelling (5\%) when exposed to the test fuels containing isobutanol. When exposed to CE25a, the vinyl ester resin specimens swelled 23\%. These resins were also significantly softened by CE25a, but the other test fuels only modestly impacted the hardness. In the dried state, these resins remained slightly softened, and more significantly the glass-to-rubber transition temperatures dropped $30^{\circ} \mathrm{C}$ or more with the fuel formations containing ethanol or isobutanol. Large declines in glass-to-rubber transition temperature are indicative of structural changes in the resins.

The acetals (POM and POM co-polymer) and PBT exhibited modest ( 5\%) swelling and softening with exposure to the test fuels. The presence of ethanol or isobutanol in the test fuel had little additional effect for the acetals. However, for PBT, Fuel C lowered the transition temperature by $30^{\circ} \mathrm{C}$, and this value declined another 10 degrees for the test fuels containing isobutanol or ethanol. PP swelled to $20 \%$ and higher with exposure to the test fuels. Most of this increase was due to Fuel C, but isobutanol did produce a small additional increase. PP was also significantly softened by the test fuels when wetted but otherwise was not affected by the test fuels. The swelling and softening results observed for PETG were similar to $\mathrm{PP}$, except that the transition temperature was lowered $70^{\circ} \mathrm{C}$ by the test fuels. This dramatic drop in temperature is a strong indicator that the test fuels (primarily Fuel C) significantly altered the molecular structure of the material. Of the plastics investigated in this study, PTU was the material most impacted by the adding of alcohol, especially ethanol. The volume increased modestly with exposure to Fuel C, CiBu16a, and CiBu24a, but CE25a caused PTU to lose 10\% volume and mass.

When dried, most of the plastic materials retained some residual test fluid in their structure, which resulted in a slight increase in the dried mass and volume compared to the original baseline values. This increase was accompanied by a small reduction in hardness. Notable exceptions were the Nylon 12, which was slightly hardened, and PTU, which was significantly hardened (embrittled).

In addition to the plastics, two rubber-impregnated cork gasket materials were also included in the material test matrix. Cork (impregnated with NBR) is commonly used as a gasket material and underwent significant volume expansion and softening when either ethanol or isobutanol was added to the base fuel. Another rubber material commonly used to impregnate cork material is epichlorohydrin rubber (ECO). This material (cork/ECO) was added to the test matrix after the specimens had already been exposed to Fuel C and CE25a, so no data were obtained for these fuels. It was, however, included in the CiBu16a and CiBu24a test fuels and significant swelling was observed. After drying, cork/NBR showed significant embrittlement with Fuel C, but less so when alcohol was added. The cork/ECO specimens only showed slight embrittlement when exposed to CiBu16a and CiBu24a and dried.

Those specimens exposed to the vapor phase of the test fuels exhibited similar behavior to those immersed in the corresponding liquid phase, albeit to a lesser extent. In fact, the fuel chemistries responsible for fracturing thermosetting resins in the liquid phase also fractured these specimens in the vapor-phase region. The implication of this finding is that materials exposed to fuel vapors are subject to the same degradation potential as materials in direct contact with liquid fuel. 


\section{Metal Results}

Very little corrosion was noted for the metallic specimens exposed to any of the test fuels. In all cases, the annualized corrosion rates based on weight loss were considered negligible for cartridge brass and undetectable for the remaining metals in the study. Surface chemistry was analyzed for the discolored specimens of cartridge brass and phosphor bronze, and the surface of each was found to contain copper sulfide. The thickness of each corrosion film was not found to be dependent on the concentration of isobutanol in the fuel blend. The results of this study confirm that 1100 aluminum, 1020 mild steel, 304 stainless steel, cartridge brass, phosphor bronze, 201 nickel, Cr-plated steel, Ni-plated steel, Cr-plated brass, Zn-plated steel, Ni-plated aluminum, and Pb-plated steel would be compatible with isobutanolcontaining fuels for the exposure conditions used in this study. 


\section{INTRODUCTION}

\subsection{BACKGROUND}

In 2011 Oak Ridge National Laboratory (ORNL) began a series of experiments to evaluate the compatibility of fueling infrastructure materials with test fuel formulations representing gasoline blended with 16 and 24\% isobutanol (by volume). This effort closely follows earlier studies performed by ORNL whereby infrastructure materials (plastics, elastomers, and metals) were exposed to standard test fuel formulations representing ethanol-gasoline fuel blends. Butamax Advanced Biofuels, LLC has developed and is marketing a technology to produce isobutanol (instead of ethanol) using existing corn-derived ethanol plants. Like ethanol, isobutanol is produced from renewable feed stocks (primarily corn). Isobutanol also has several attractive qualities relative to ethanol; namely, it has a higher energy density, is relatively insoluble to water, and has less polarity. The implication is that isobutanol offers improved fuel economy, while exhibiting equal to or better compatibility potential to fuel system materials. One pathway to the introduction of isobutanol as a transportation fuel is through an existing waiver from the U.S. Environmental Protection Agency (EPA). The Texas Methanol Corporation received a waiver from the EPA to allow for a gasoline-alcohol fuel blend containing up to $3.7 \mathrm{wt} \%$ oxygen. ${ }^{1}$ This value corresponds to an ethanol volume concentration of $10 \%$ and an isobutanol content of $16 \%$ by volume. More recently, the EPA approved 15\% ethanol to be blended into gasoline as an acceptable fuel. ${ }^{2}$ (The oxygen equivalent of E15 for isobutanol is $24 \%$.) Because isobutanol can be blended at higher volumes than ethanol under the OCTAMIX waiver, isobutanol offers a viable means of increasing renewable fuel utilization toward compliance with the Energy Independence and Security Act.

Prior to this investigation, ORNL had performed compatibility studies on fueling infrastructure materials exposed to standard test fuels representing various blends of ethanol and gasoline. ${ }^{3,4,5}$ A wide range of ethanol concentrations were evaluated, but the test fuels most relevant to this study are Fuel C, CE10a, CE17a, and CE25a. The results for CE10a and CE17a were used to provide oxygen-equivalent comparisons to test fuels in this study containing 16 and 24\% isobutanol, respectively. CE17a was selected as representative of E15 since surveys indicated that the actual concentration of ethanol in E10 dispensers has been noted to vary by as much as $2 \%$. Therefore, $17 \%$ aggressive ethanol was selected as a worse-case composition. Elastomer and metallic materials were evaluated in all three of these fuels, however plastic specimens were only exposed to CE25a. (Plastics were not included as part of the CE10a or CE17a studies because the original study scope only included metals and elastomers.) These fuels were based on the Fuel C composition and aggressive ethanol formulations described in Society of Automotive Engineers (SAE) J1681, "Gasoline, Alcohol, and Diesel Fuel Surrogates for Materials Testing." ${ }^{6}$ Fuel C is a 50-50 mixture of toluene and isooctane and is representative of highly aromatic gasoline ( $>40 \%$ aromatics by volume). The "E" designation following the $C$ refers to ethanol and the numbers that follow CE refer to the volume fraction of ethanol added to Fuel C, while the small letter "a" following the numbers refers to an aggressive ethanol formulation (containing water and trace levels of sodium chloride, acetic and sulfuric acids specified in SAE J1681).

Butamax Advanced Biofuels is in the process of licensing their technology to corn ethanol plants in the United States for full-scale production of isobutanol. However, before isobutanol becomes an accepted fuel, it is necessary to demonstrate that its compatibility to fuel storage and delivery systems is no worse than that for ethanol. It was in this context that ORNL was approached by Butamax to perform a series of similar compatibility experiments in parallel with a solubility study at Underwriters Laboratories to evaluate isobutanol compatibility to fuel infrastructure materials. The materials used in this study were identical to those used in the ethanol studies. However, six of the eight original fluorocarbons and fluorinated HDPE were not included since they were sufficiently similar to other fluorocarbons and HDPE in the ethanol exposure studies. 


\subsection{ISOBUTANOL}

Isobutanol is one of four isomers of butanol, and it has the chemical formula of $\left(\mathrm{CH}_{3}\right)_{2} \mathrm{CHCH}_{2} \mathrm{OH}$ and its molecular structure (compared to ethanol) is depicted in Fig. 1. As a biofuel isobutanol has several inherent advantages over ethanol. It has a higher energy density than ethanol, has lower oxygen mass fraction, and is less polar. This reduced polarity suggests that solubility (and therefore compatibility) to polymers should either be similar or better than ethanol exposures. Another attractive feature is that isobutanol is relatively insoluble to water, whereas ethanol is completely soluble at most ambient conditions.

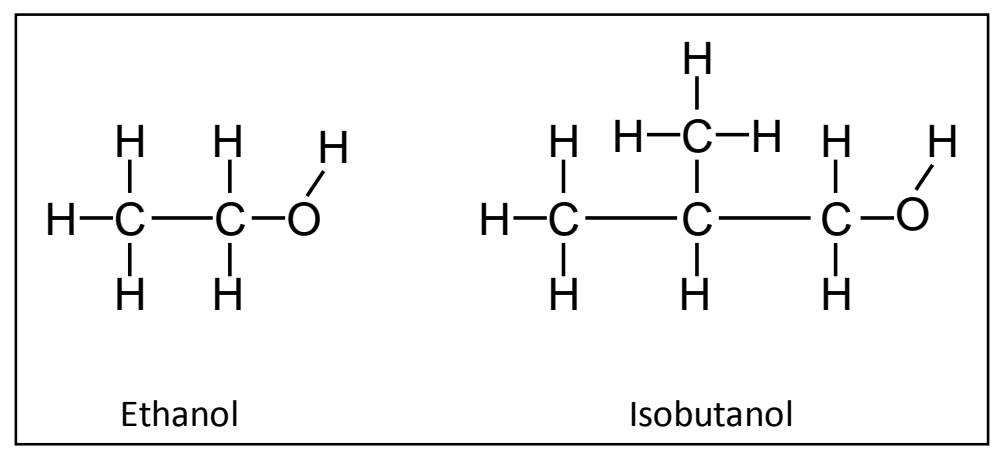

Fig. 1. Molecular diagrams of ethanol and isobutanol.

Vehicle studies with isobutanol blends have been conducted with no noticeable issues on vehicle performance or fuel system compatibility. ${ }^{7}$ More recently, Butamax completed a study examining permeation emissions in gasoline vehicle fuel systems and found that, when added to gasoline, isobutanol will reduce gasoline evaporative emissions. ${ }^{8}$

\subsection{AGGRESSIVE ISOBUTANOL FORMULATION}

At the start of this effort, no standard aggressive test fuel for gasoline-isobutanol blends existed for either the Society of Automotive Engineers (SAE) or the American Society for Testing and Materials (ASTM). Test fuels representing oxygenated gasoline are described in SAE J1681 for material compatibility evaluations and the aggressive ethanol composition was used as the basis for the construction of an analogous aggressive isobutanol formulation. ${ }^{1}$ Aggressive ethanol contains $99 \%$ ethanol, $1 \%$ water, 5 ppm sodium chloride, 25 ppm sulfuric acid and, 75 ppm acetic acid. The components making up a corresponding aggressive isobutanol solution were kept similar to aggressive ethanol, except that isobutanol replaced ethanol and isobutyric acid was substituted for acetic acid.

The formulations for the aggressive methanol and ethanol formulations in SAE J1681 indicate that the molar concentration of the organic acid was kept constant at $0.001 \mathrm{M}$ for both alcohol types. Therefore, in order maintain consistency with the protocol in SAE J1681, a molar ratio of $0.001 \mathrm{M}$ was used to determine the concentration of isobutyric acid in an aggressive isobutanol formulation. By keeping the molar concentration constant, the number of acid protons in a given volume of test fuel is the same for each aggressive alcohol.

The resulting composition used to make 1 liter of the aggressive isobutanol is shown in Table 1 . The concentrations of water, sodium chloride, and sulfuric acid matched that of aggressive ethanol, since the processes and handling of isobutanol and ethanol are expected to be similar. 
Table 1. Formulation used to make $1 \mathrm{~L}$ of aggressive isobutanol

\begin{tabular}{lcc}
\hline \multicolumn{1}{c}{ Component } & Mass (g) & Concentration (\%) \\
\hline Reagent-grade isobutanol & 797.7 & 99 \\
Deionized water & 7.987 & 0.99 \\
Sodium chloride & 0.004 & $5 \mathrm{ppm}$ \\
Sulfuric acid & 0.021 & $25 \mathrm{ppm}$ \\
Isobutyric acid & 0.088 & $109 \mathrm{ppm}$ \\
\hline
\end{tabular}

\subsection{PROPERTIES RELATIVE TO POLYMER-FUEL COMPATIBILITY}

The compatibility of a polymeric material typically refers to the solubility of the polymer to a particular solvent. It can also mean susceptibility to chemical attack, although the polymers and test fuels evaluated in this study are not considered to be chemically reactive with each other. Solubility is typically assessed in polymers by measuring the volume swell of the polymer exposed to the solvent of interest. ${ }^{9,10,11}$ Swell is almost always accompanied by a decrease in hardness (softening) that also affects performance. For elastomer materials hardness is measured using the Shore A method, while for plastics it is the Shore D method.

Elastomers are a class of polymers which are predominantly used in sealing applications, and most o-ring and seal manufacturers provide compatibility tables of their products with various solvents including ethanol, toluene and isooctane. ${ }^{12,13}$ These tables include a ranking corresponding to the level of volume swell associated with exposure to a particular solvent.

For seal applications, shrinkage of the elastomer upon drying is also a critical parameter since a contraction of volume can conceivably enable leakage to occur. Shrinkage is also indicative of the removal of one or more components of the elastomers (by the solvent). This extraction of additives can negatively change the properties of the elastomer, leading to reduced performance and durability. For most seal applications, some level of volume swell is acceptable, since the expansion will serve to maintain the seal. ${ }^{14}$ The actual acceptable level of swell is dependent on the particular application. It is known that excessive swell can lead to extrusion of the elastomer beyond the sealed interface where it becomes susceptible to damage. Also, since high swell is indicative of high solubility, there is a heightened potential for fluid to permeate through the seal and, subsequently be released into the environment.

Plastics, on the other hand, are used primarily in structural applications, such as piping and fluid containment. Volume change, especially in a rigid system, may create internal stresses which may negatively affect performance.

In order to better understand and predict the compatibility for a given polymer type and fuel composition, an analysis based on Hansen solubility theory was performed for each plastic and elastomer material. From this study, the solubility distance was calculated for each polymer material and test fuel combination. Using the calculated solubility distance, the ethanol concentration associated with peak swell and overall extent of swell can be predicted for each polymer.

The other test method used in this study to assess compatibility was dynamic mechanical analysis (DMA), which measures the storage modulus as a function of temperature. ${ }^{15}$ DMA is important for understanding how a polymer will behave at a given temperature. All polymers will undergo microstructural relaxation, whereby they transition from a rigid glass state to a rubbery pliable state. The 
temperature associated with the onset of this transition is called the onset of the glass-to-rubber transition temperature, or transition temperature. This parameter is important since any shift in the glass-to-rubber transition temperature is indicative of structural changes which may not be revealed in the swell and hardness measurements. 


\section{MATERIALS}

A survey was performed as part of the earlier investigation on ethanol-gasoline fuels to compile a list of materials used in underground storage and fuel delivery systems. ${ }^{3-5}$ These materials included polymers (plastics and elastomers) and metal alloys. A diagram identifying the location and identification of components and materials is shown in Fig. 2. This list was developed through discussions with component materials suppliers, literature surveys, and industry experts.

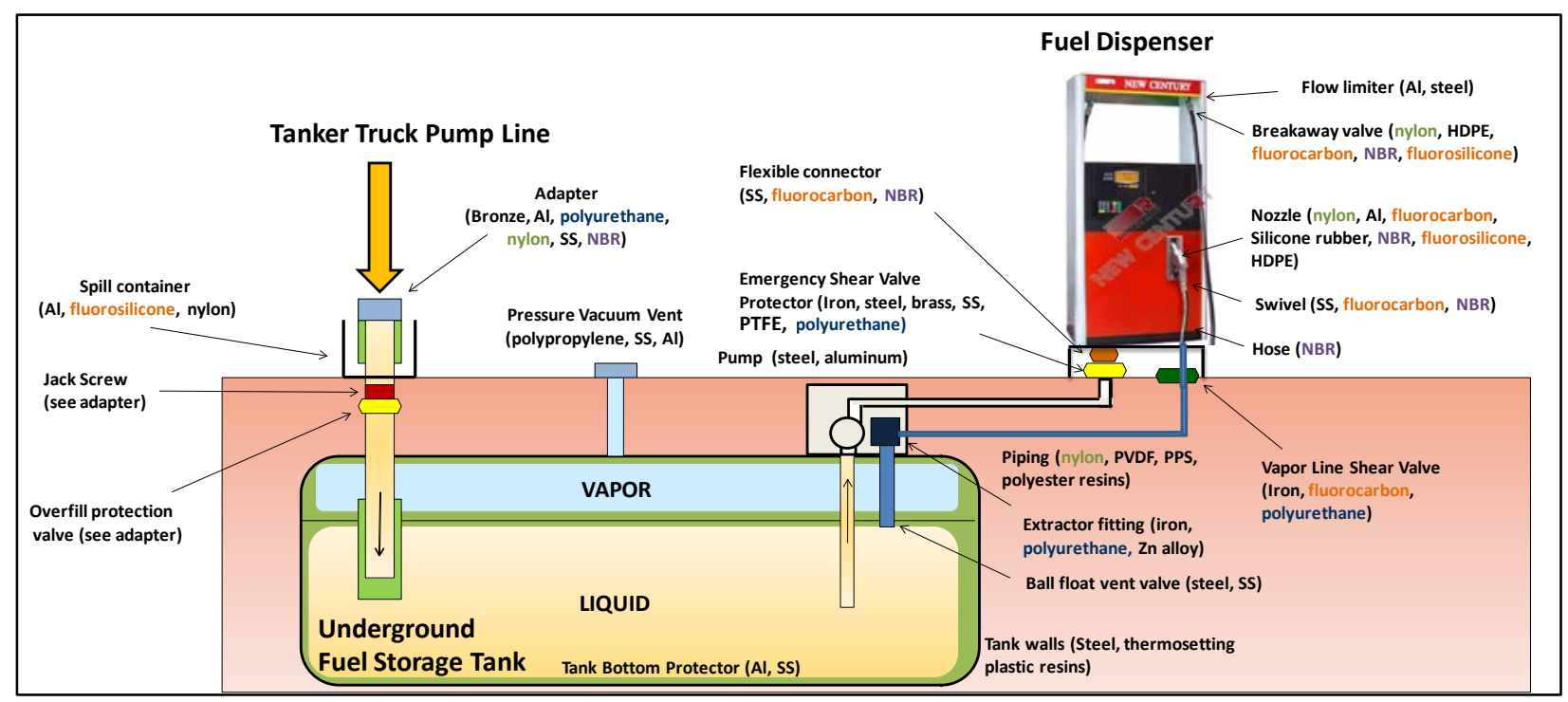

Fig. 2. Fueling infrastructure components and materials.

As shown in Fig. 2, there exists a substantial degree of commonality among materials used in many of the components and subsystems. In general, metals and plastics are used in structural applications (such as tanks or piping) and as components of pumps, valves, swivels, and fittings. Elastomers are used primarily in sealing applications (such as o-rings and gaskets) or as hose material. Rubberized cork gaskets are ubiquitous in legacy dispensers and occasionally present in newer units. They are typically impregnated with either NBR or epichlorohydrin (ECO) to improve durability and plasticity. A complete listing of the material types included in this study (for each of the three primary material classes) is shown in Table 2.

\subsection{SELECTION AND DESCRIPTION OF ELASTOMERIC MATERIALS}

The elastomer results presented and discussed in this report are an expansion of an earlier effort that focused on evaluating elastomer performance with gasoline test fuels containing up to $25 \%$ aggressive ethanol. These earlier results were published in a report ${ }^{1}$ that includes a more detailed discussion of the literature, materials, experimental procedure, and results. The elastomeric materials evaluated in the present study are:

1. two types of fluorocarbon rubbers (Viton A401C and Viton B601),

2. one fluorosilicone rubber,

3. one silicone rubber,

4. six acrylonitrile-butadiene rubbers (NBRs),

5. one styrene-butadiene rubber (SBR),

6. one neoprene rubber, and

7. one polyurethane rubber. 
Table 2. Complete list of materials examined in the study

\begin{tabular}{l||l|l}
\hline \multicolumn{1}{c||}{ Metals \& Alloys } & \multicolumn{1}{c|}{ Elastomers } & \multicolumn{1}{c}{ Plastics } \\
\hline 304 stainless steel & Fluorocarbon (2 grades) & High density polyethylene \\
1020 carbon steel & NBR (6 grades) & Polypropylene \\
1100 aluminum & Silicone rubber & Polyoxymethylene (2 types) \\
Cartridge brass & Fluorosilicone rubber & Nylon (4 grades) \\
Phosphor bronze & Neoprene rubber & Polyvinylidene fluoride \\
Nickel 201 & Styrene butadiene rubber (SBR) & Polytetrafluoroethylene (Teflon) \\
Terne-plated steel & Polyurethane & Polyphenylene sulfide \\
Galvanized steel & Rubberized cork (2 types) & Polyethylene terephthalate (2 types) \\
Cr-plated brass & & Polybutylene terephthalate \\
Cr-plated steel & & Polythiourea \\
Nickel-plated aluminum & & Isophthalic polyester resin (2 types) \\
Nickel-plated steel & & Terephthalic polyester resin \\
& & Novolac vinyl ester resin \\
& & Epoxy resin (2 types and 2 curing methods) \\
\hline
\end{tabular}

The two fluorocarbons were provided by one supplier, while the NBRs were supplied by two manufacturers. The six grades of NBR evaluated in this study are designed for use as fuel lines or hoses. For each elastomer material, three specimens were exposed directly to the test fuel liquids and one was exposed to the test fuel vapors. The length, width, and thickness of each specimen were 3.8, 1.3, and $0.2 \mathrm{~cm}(1.5,0.5$, and 0.08 in.), respectively.

\subsection{SELECTION AND DESCRIPTION OF PLASTIC MATERIALS}

Plastic materials are divided into two classes: thermoplastics and thermosets (or thermosetting resins). Thermoplastic polymers do not undergo a chemical change in composition when heated, though they do soften or melt. When cooled they typically return to their original composition, and consequently, they can be molded repeatedly. Thermosets, on the other hand, can only be cured and shaped once. After forming, they remain in a solid (highly rigid) state and cannot be melted. In the thermosetting process, the chemical reaction forming the cross-linked polymer is not reversible. A complete listing of the plastic materials according to type and application is shown in Table 3. For each material listed in Table 3 a total of four specimens were evaluated. Of these, three were exposed directly to the test fuel liquids and one was exposed to the vapor-phase region. Each specimen measured $2.54 \mathrm{~cm}$ (1 in.) wide, $7.6 \mathrm{~cm}$ (3 in.) long, and $0.32 \mathrm{~cm}(0.125 \mathrm{in}$.) thick.

\subsubsection{Thermoplastics}

Thermoplastics are usually pliable, and as a result, they are used in the construction of flexible piping systems. The more chemically resistant grades are also used as high performance seals. The thermoplastic materials examined in this study are grouped according to their application and type as shown in Table 3. The first group includes those used as permeation barriers and liners in flexible piping systems. This group includes polyphenylene sulfide (PPS), polyethylene terephthalate (PET), polyvinylidene fluoride (PVDF), and polytetrafluoroethylene (PTFE). PTFE (also known as Teflon) is also used in sealing applications as well. PET (or Mylar) is the most commonly used barrier plastic, primarily because it has a lower cost than the other three materials. 
Table 3. Categories and types of plastic materials examined in the study

\begin{tabular}{l||c}
\hline \multicolumn{1}{c||}{ Thermoplastics } & \multicolumn{1}{c}{ Thermosets } \\
\hline Permeation barrier materials & Polyester resins \\
PPS, PET, PTFE, \& PVDF & Isophthalic polyester (2 types) \\
& Terephthalic polyester (1 type) \\
& Vinyl ester resin \\
Nylons and HDPE & \\
Nylon 6, Nylon 6,6, Nylon 12, \& Nylon 11 & \\
HDPE & Epoxy resins (2 curing conditions) \\
Other common plastics & \\
Acetals: POM (homopolymer) \& POM (copolymer) & \\
Polyesters: PBT \& PETG (PET copolymer) & \\
Polypropylene (PP) & \\
Polythiourea (PTU) &
\end{tabular}

The second grouping includes nylon and HDPE, which are also used in flexible piping systems. These materials do not have the permeation resistance (or high cost) of the four barrier materials, but they do exhibit good compatibility and are used to reinforce and support flexible piping, either as braided fiber bundles or as high strength secondary wall materials. Four grades of nylon were included. Nylons 6 and 6,6 are similar to each other and have good mechanical properties. Nylons 12 and 11 are also used in flexible piping. It is worth noting that Nylon 11 is manufactured from vegetable oil, while the other three are derived from petroleum.

The third grouping includes those plastics which, while not identified by the survey results as infrastructure materials, are so widely utilized that the likelihood is high that they are, in fact, used in some infrastructure applications. These materials include acetal (POM), polybutylene terephthalate (PBT), polyethylene terephthalate co-polymer (PETG), polypropylene (PP), and polythiourea (PTU).

\subsubsection{Thermosets (or Resins)}

Unlike thermoplastics, thermosets can only be cured and shaped once. After forming, they remain in a solid (highly rigid) state and cannot be melted. Heating will result in oxidation and thermal breakdown rather than softening. In the thermosetting process, the chemical reaction forming the cross-linked polymer is not reversible. Thermosets are used in rigid applications, especially as resins in fiberreinforced plastics (FRPs), and as adhesives to bond flanges and pipe sections. FRPs are used extensively in fuel storage applications and rigid piping systems.

The thermosets examined in this study included two types of polyester resins (isophthalic and terephthalic polyesters). These resins represent legacy and current resins used in the construction of underground storage tanks and FRP systems. The two types of isophthalic resins differed according to the ratio of isophthalic acid to maleic anhydride. One formulation has a 1:1 ratio of isophthalic acid to maleic anhydride and is representative of resins used in FRP systems (including underground storage tanks) prior to 1990. The other isophthalic polyester resin has a 1:2 ratio of isophthalic acid to maleic anhydride and was introduced during the 1990s for use with FRP systems. The terephthalic acid polyester resin has a 1:1 ratio of terephthalic acid to maleic anhydride and was also introduced in the 1990s for use in FRP systems. A vinyl ester resin material was also included which is representative of a newer highperformance formulation used in the construction of FRP systems.

It is important to note that the thermoset specimens consisted of pure resin only. In actuality, these resins are never used without some level of fiber reinforcement, which serves to constrain expansion and 
increase fracture resistance, strength, and durability. Therefore, the performance of pure resins to the test fuels does not necessarily correspond directly to the actual reinforced samples. However, should the resin become degraded, the composite itself will be less durable. It is important to note that in addition to being used as the matrix material in FRP, these resins may also be used as adhesives to connect piping and flanges.

An epoxy resin that was cured under two different temperatures, room temperature and elevated temperature, was also examined. There are a multitude of epoxy resin types as well as curing conditions; all variations could not be included, so one formulation and two different processing conditions were examined as representative examples.

\subsection{SELECTION AND DESCRIPTION OF METALLIC MATERIALS}

Metals are used ubiquitously in fueling infrastructure, and a list of those alloys selected for evaluation is shown in Table 4. The two most commonly used metallic materials are steel and aluminum alloys. Steel is commonly used in the construction of underground storage tanks and piping systems, and aluminum is used in submersible turbine pumps, valves, and nozzles. Other metallic materials, such as bronze, brass, and nickel are used in connections, valves, swivels, etc.

Table 4. List of single and plated metallic materials examined in the study

\begin{tabular}{l||l}
\hline \multicolumn{1}{c||}{ Bare Metal Specimens } & \multicolumn{1}{c}{ Fully Plated Specimens } \\
\hline 1020 carbon steel & Nickel-plated aluminum \\
1100 aluminum & Nickel-plated steel \\
Cartridge brass & Chromium-plated brass \\
Phosphor bronze & Chromium-plated steel \\
201 nickel & Zinc-plated (“galvanized”) steel \\
304 stainless steel & Lead-plated (“terne”) steel \\
\hline
\end{tabular}

In addition to exposure of coupons with a fully intact plating, coupons of each type were exposed with the plating partially removed (using 120 grit sandpaper) to generate a galvanic couple between the relatively noble (passive) plating material and the relatively active (anodic) exposed substrate material. Figure 3 shows representative specimens of plated material with part of the plating removed. The specimens were $5.1 \mathrm{~cm}$ (2 in.) long, $1.9 \mathrm{~cm}$ (0.75 in.) wide, and $0.32 \mathrm{~cm}(0.125 \mathrm{in}$.) thick, with the exception of the bronze and terne specimens, which were $0.16 \mathrm{~cm}$ (0.0625 in.) thick. 


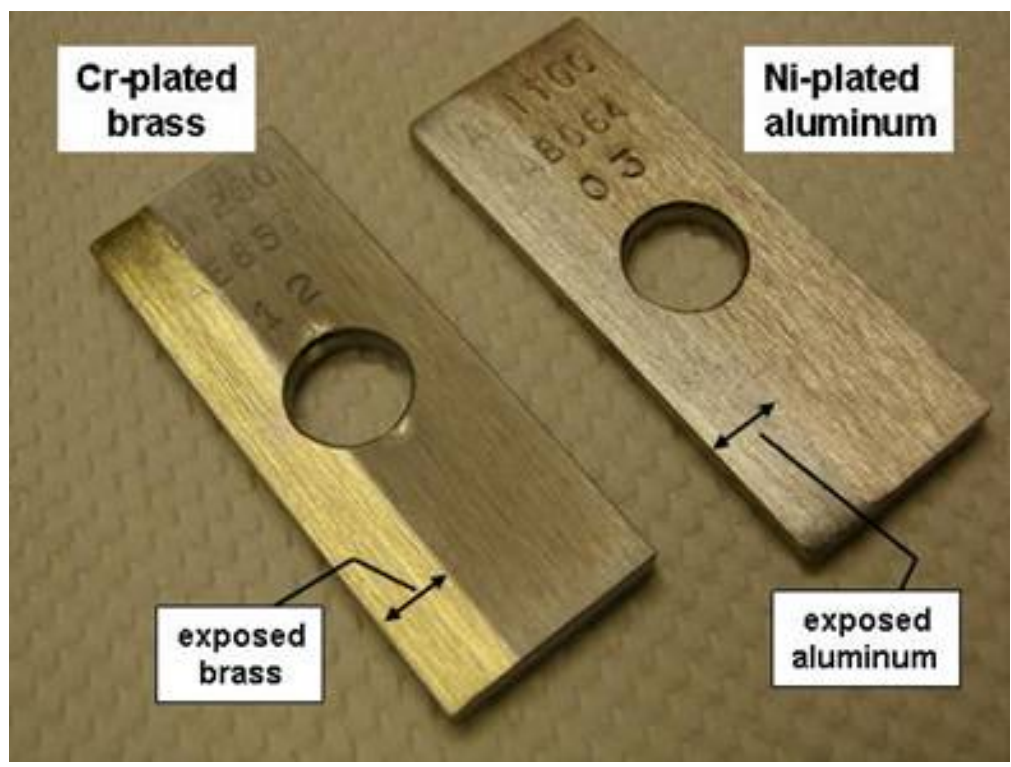

Fig. 3. Appearance of galvanically coupled specimens showing exposed surfaces.

For each metal type, two specimens were mounted on brackets as shown in Fig. 4. This arrangement was similar to the polymer specimens, except that for the metal specimens, Teflon shoulder washers were used to prevent contact with the metal mounting hardware and slotted crevice washers were placed on one side of each specimen to provide a site for the analysis of crevice corrosion, should it occur. In addition to these two specimens mounted on a single bracket, an additional specimen was placed in the headspace for comparison.

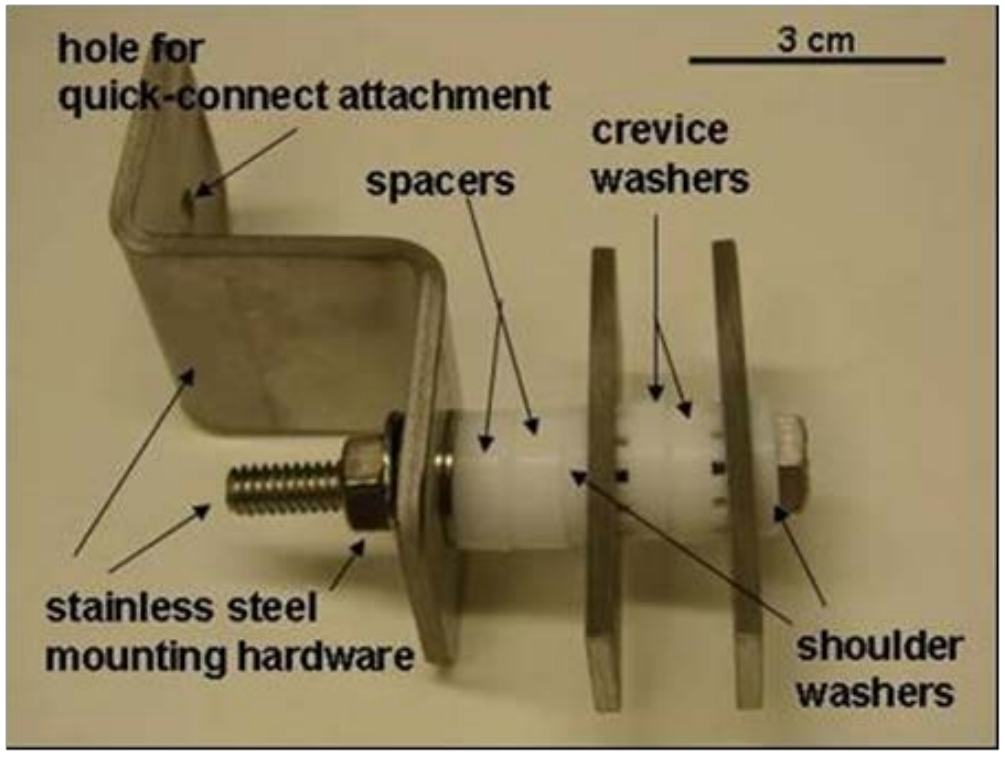

Fig. 4. Mounted metal specimens and mounting hardware. Each fixture held duplicate metallic specimens isolated from each other and the stainless steel hardware by Teflon components. 



\section{TEST FUELS}

In this study test fuels representing gasoline blended with $16 \%$ and $24 \%$ isobutanol were prepared. These fuels, denoted as CiBu16a and CiBu24a, were based on Fuel C using the aggressive isobutanol formulation described in the introduction. From the earlier ethanol blend compatibility study, the results from the Fuel C, CE10a, CE17a, and CE25a exposures were included for comparison and analysis. Although CE17a contains 17\% ethanol, it is used to represent E15 since field surveys on E10 have shown that ethanol content in gasoline fuels can vary by several percentage points. ${ }^{16}$

The aggressive formulation is conservative by design but is considered to be representative of field conditions since organic acids such as formic and acetic acid are present in certain fuels, including ethanol. These acids are formed in the production process of ethanol or created via oxidation during handling, transfer, or storage. Sulfuric acid is believed to originate from impurities associated with alcohol fermentation, but it may also be formed by the reaction of fuel-borne sulfur with alcohol and can be particularly corrosive to metals and polymers. Commercial-grade gasoline may contain varying amounts of sulfur, which is usually present as disulfides. Disulfides are converted to sulfonic acids in the presence of atmospheric oxygen and water. Since water is generally present either as a liquid or as vapor, sulfuric acid will form in ethanol-blended gasoline and possibly in isobutanol blends as well.

These test fuels are designed to simulate severe, real-world conditions. They are also intended to minimize the exposure time necessary to rigorously evaluate materials while providing a standard method of testing fuel system materials. Fuel $\mathrm{C}$ was selected as the control since it represents premium gasoline and is a widely used standard test fluid for studying material compatibility to gasoline.

Because plastics were added to the material listing at a later point in the ethanol compatibility study, they were not included in all of the ethanol-gasoline fuel blends. The material and fuel test matrix in Table 5 shows that elastomer and metals were evaluated in all of the test fuel formulations, but the plastics were only exposed to Fuel C, CE25a, CiBu16a, and CiBu24a.

Table 5. Matrix of material types and corresponding test fuels

\begin{tabular}{lcccccc}
\hline \multirow{2}{*}{ Material Type } & \multicolumn{7}{c}{ Test Fuel Formulation } \\
\cline { 2 - 7 } & Fuel C & CE10a & CE17a & CE25a & CiBu16a & CiBu24a \\
\hline Elastomers & $\mathrm{X}$ & $\mathrm{X}$ & $\mathrm{X}$ & $\mathrm{X}$ & $\mathrm{X}$ & $\mathrm{X}$ \\
Metals & $\mathrm{X}$ & $\mathrm{X}$ & $\mathrm{X}$ & $\mathrm{X}$ & $\mathrm{X}$ & $\mathrm{X}$ \\
Plastics & $\mathrm{X}$ & & & $\mathrm{X}$ & $\mathrm{X}$ & $\mathrm{X}$ \\
\hline
\end{tabular}

The test fuels were prepared by splash-blending the components one at a time. The first step was to prepare the aggressive water solution, which was poured into an empty 30-gal drum. Reagent-grade isobutanol was added to the aggressive water solution followed by the appropriate volume of Fuel C. The final fuel formulation was poured into the dynamic chamber, which had been preloaded with the material specimens. Visual observation indicated that the resulting fuel mixture was single phase. In order to maintain a constant humidity in the vapor space, each chamber was purged with dry air before being sealed. 



\section{EXPERIMENTAL PROTOCOL}

Two identical evaluation chambers were constructed to control the fluid flow rate and temperature under sealed conditions. A schematic diagram showing the chamber assembly is shown in Fig. 5. Each chamber is capable of exposing a large number of polymer and metal specimens to the test fuel. The specimens were attached to mounting brackets, which were affixed to the inside surface of a cylindrical liner placed within each vessel. The photograph in Fig. 6 shows a set of mounted specimens arranged inside one of the test chambers. To achieve dynamic flow, each chamber was equipped with a paddle to impart a rotating fluid flow at a rate of $0.8 \mathrm{~m} / \mathrm{s}$ past the specimens. These chambers were sealed to prevent fuel leakage and employed a heating jacket to maintain a constant temperature of $60^{\circ} \mathrm{C}$ during the exposure period. Each container was filled to a predetermined level with each test fuel. The majority of the specimens were completely submerged in the test fuel liquid, while a second set of specimens were positioned above the liquid fuel line in the headspace for exposure to the vapor-phase environment.

A flow chart highlighting the treatments and measurements for each material type is shown in Fig. 7. The elastomeric and metallic specimens were exposed for a period of 4 weeks, while the plastic materials (and rubberized cork samples) were kept in the chambers for 16 weeks. Because plastics have a denser molecular structure than elastomers, they require a longer exposure period to achieve saturation. (They also required a longer drying time.) The polymer specimens submerged in the liquid phase were removed and measured for volume, mass, and hardness while in the wetted (or saturated) state. Once the wetted properties were measured, the elastomer and cork specimens were dried at $60^{\circ} \mathrm{C}$ for 20 hours, whereas the plastics were dried at $60^{\circ} \mathrm{C}$ for a total of 65 hours. After drying, each specimen was once again measured for volume, mass, and hardness. The changes in these properties from the baseline (untreated) condition were used to assess compatibility. In contrast, the specimens exposed to the vapor phase were measured only for hardness in the unexposed original condition and after drying at $60^{\circ} \mathrm{C}$ for 65 hours. The metal specimens were measured for mass loss, and in some cases the surface chemistries were also analyzed. DMA testing was also performed to evaluate whether any structural changes had taken place in the polymers following exposure to the test fuels.

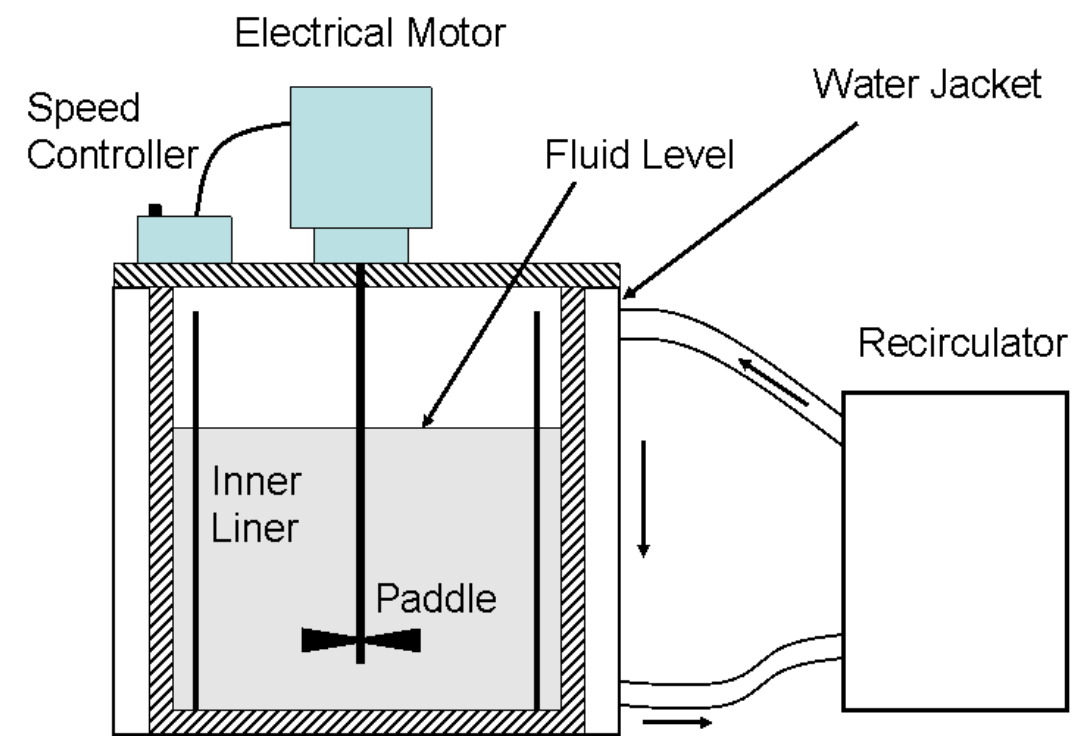

Fig. 5. Arrangement and components of dynamic environmental chamber. 


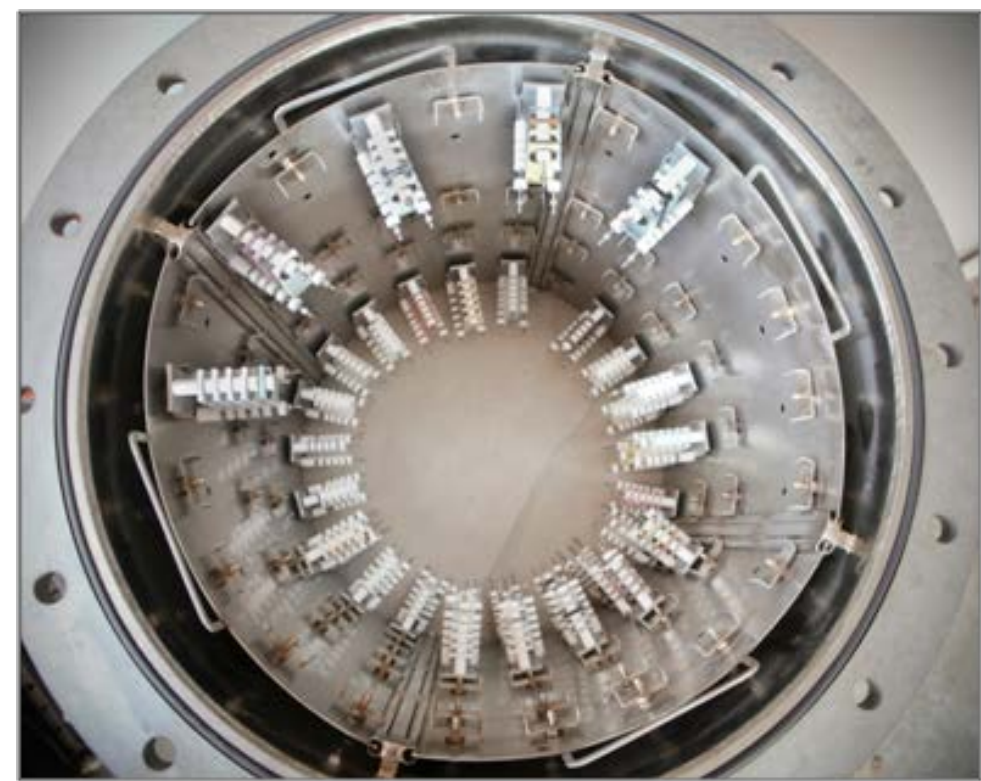

Fig. 6. Plan view of the interior of the environmental chamber showing arrangement of specimens and the liner. Specimens are shown attached to the liner.

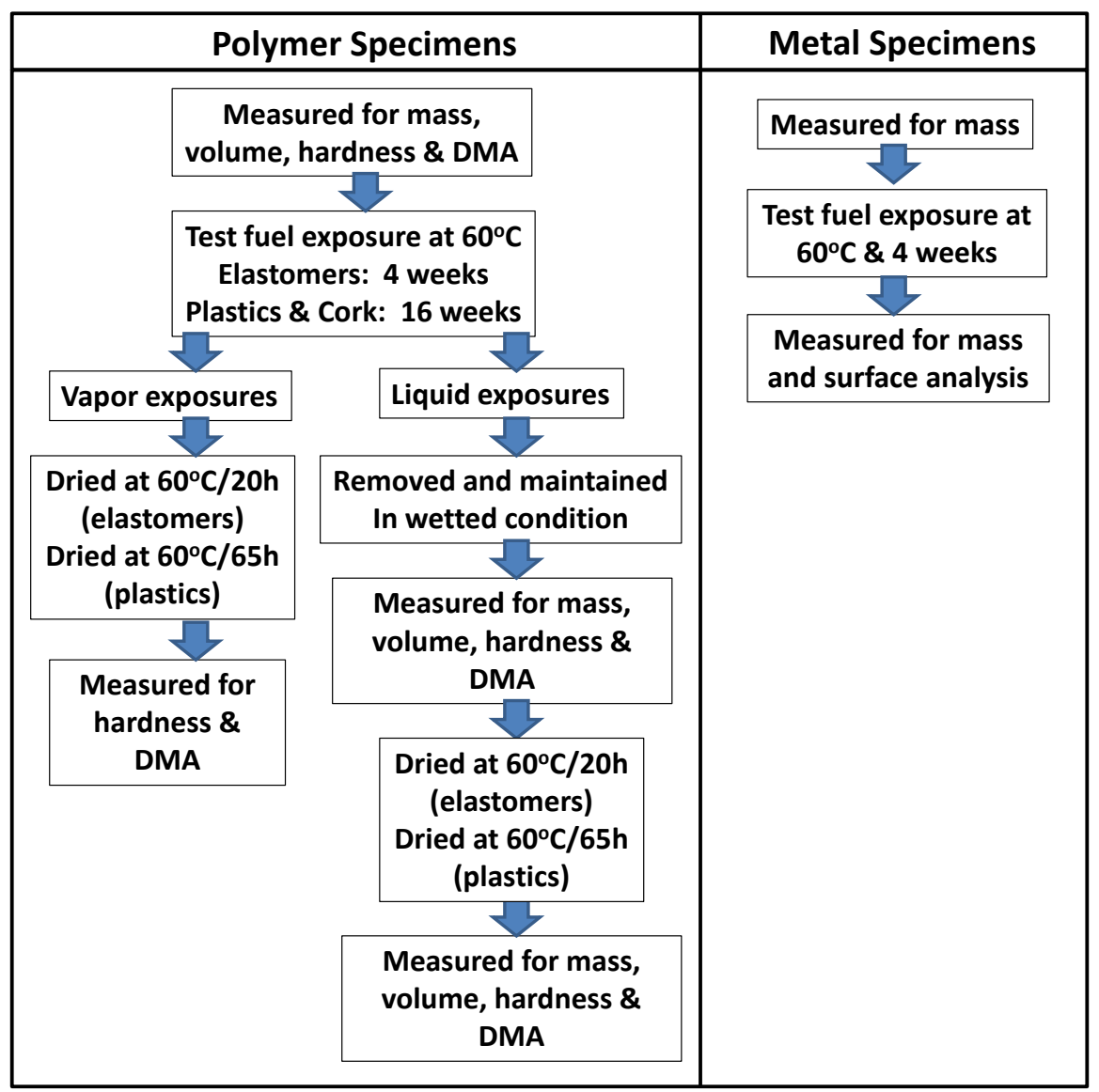

Fig. 7. Flowchart outlining test and measurement protocols for each material type. 


\section{SOLUBILITY ANALYSIS}

A solubility analysis was performed for the polymeric materials (elastomers and plastics) with representative isobutanol-gasoline fuel blends in order to predict compatibility, or more precisely the relative extent of volume swell, for each plastic type as a function of fuel chemistry. (High solubility is an indicator of poor compatibility.) The analytical approach was based on the Flory-Huggins model using the Hansen solubility parameter (HSP) methodology. ${ }^{9}$ Previously this type of analysis was performed to better understand and predict swelling behavior in polymeric materials exposed to blends of gasoline and ethanol and was useful in predicting the location of maximum swell and relative extent of volume expansion. Relative volume swell was assessed by determining the solubility distance $\left(d_{S}\right)$ for each material type as a function of alcohol content and comparing these values to the interaction radius $\left(I_{R}\right)$ of the polymer. The interaction radius represents the zone of high solubility for a given polymer and is independent of solvent type. In other words, if the solubility distance falls within (or is less than) the interaction radius, then high solubility can be expected. The relationship between $I_{R}$ and $d_{S}$ is a useful means for predicting the extent of solubility of a particular material-fuel combination as depicted in Table 6.

\begin{tabular}{cc}
$\begin{array}{c}\text { Table 6. Volume swell (or solubility) relationship } \\
\text { with interaction radius and solubility distance }\end{array}$ \\
\hline Relationship & $\begin{array}{c}\text { Predicted Extent of } \\
\text { Solubility/Volume Swell }\end{array}$ \\
\hline $\mathrm{d}_{\mathrm{S}}<<\mathrm{I}_{\mathrm{R}}$ & High \\
$\mathrm{d}_{\mathrm{S}}<\mathrm{I}_{\mathrm{R}}$ & Moderate to High \\
$\mathrm{d}_{\mathrm{S}}=\mathrm{I}_{\mathrm{R}}$ & Low to Moderate \\
$\mathrm{d}_{\mathrm{S}}>\mathrm{I}_{\mathrm{R}}$ & Low \\
$\mathrm{d}_{\mathrm{S}}>>\mathrm{I}_{\mathrm{R}}$ & Insoluble \\
\hline
\end{tabular}

The solubility distance $\left(\mathrm{d}_{\mathrm{s}}\right)$ can be viewed as a measure of the thermodynamic similarity between the polymer and a particular solvent (i.e., test fuel). Because $d_{S}$ is a measure of chemical similarity between a solid and liquid, the relative extent of swelling in the solid will be inversely related to the value of $d_{s}$. For example, if the solubility distance is two times larger in Fuel C than in Fuel A, it is expected that the polymer volume will swell twice as high in Fuel A than in Fuel C. A key limitation of this exercise is that the HSPs (and interaction radii) were obtained from literature-derived sources and, therefore, may not precisely match the values for the tested materials.

\subsection{ELASTOMERS}

The solubility distance for each material was calculated as a function of isobutanol concentration in Fuel C, and the resulting curves for the six elastomer types are shown in Figs. 8-13. The corresponding results for gasoline-ethanol blends are included in the curves for comparison. The interaction radius for each material is represented by the dashed horizontal line in each graph. In general, when starting from Fuel C ( $0 \%$ alcohol) the solubility distance decreased with alcohol concentration and reached a minimum value somewhere between 20 and 60\% alcohol concentration. However, for the fluoroelastomer and polyurethane materials, $d_{\mathrm{S}}$ values were observed to decline almost continuously with isobutanol content. 


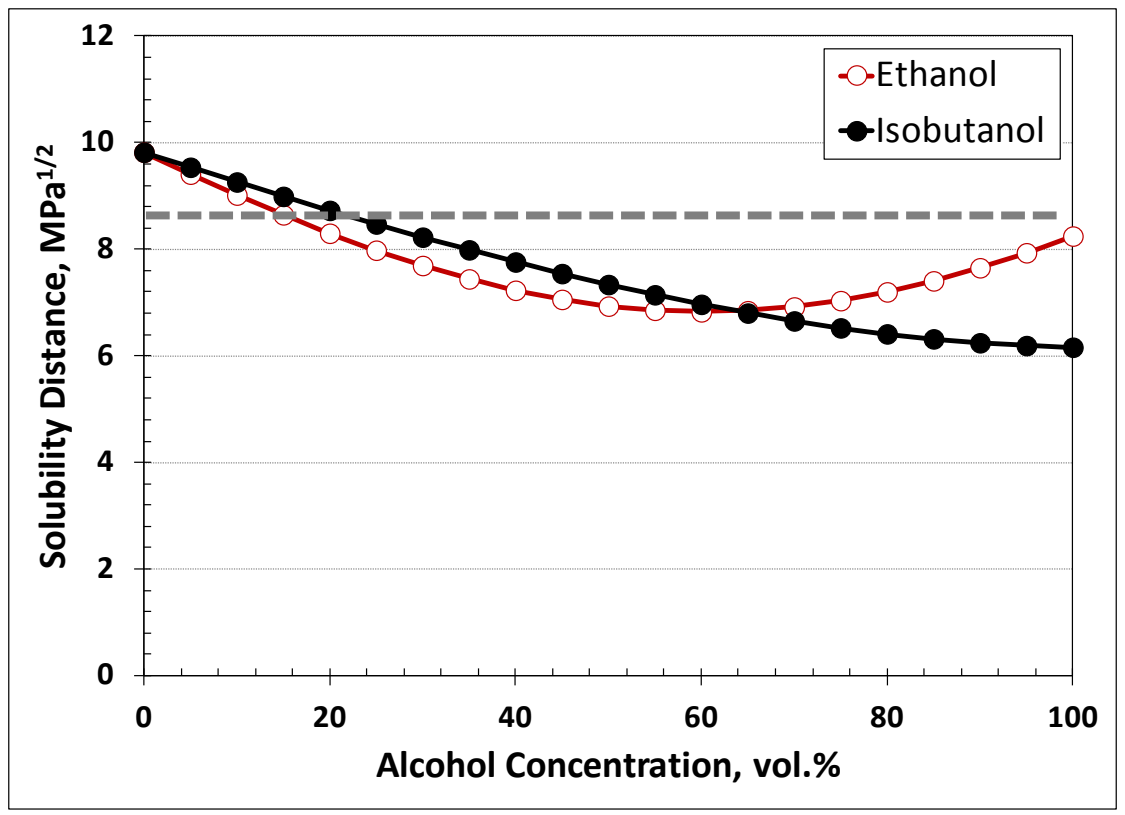

Fig. 8. Solubility distance curves for fluoroelastomers for each alcohol type and concentration. The interaction radius for typical fluoroelastomers is 8.8 $\mathrm{MPa}^{1 / 2}$.

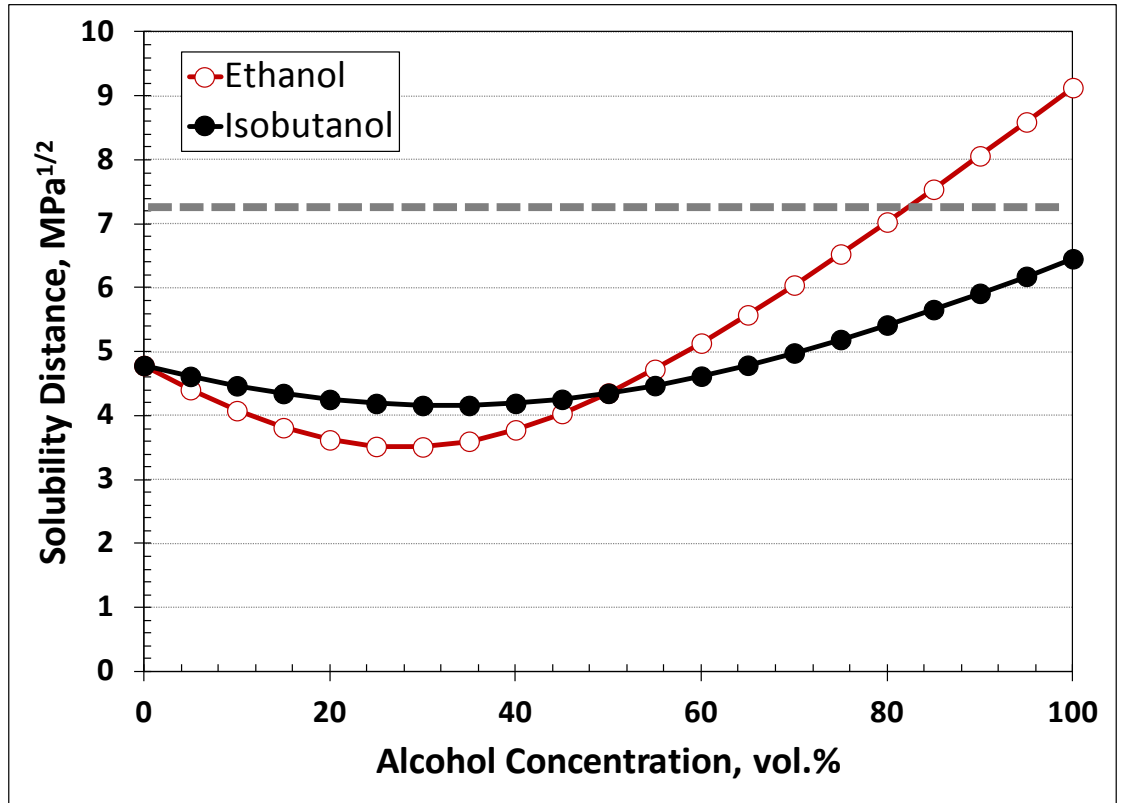

Fig. 9. Solubility distance curves for NBR for each alcohol type and concentration. The interaction radius for typical NBRs is $7.3 \mathrm{MPa}^{1 / 2}$. 


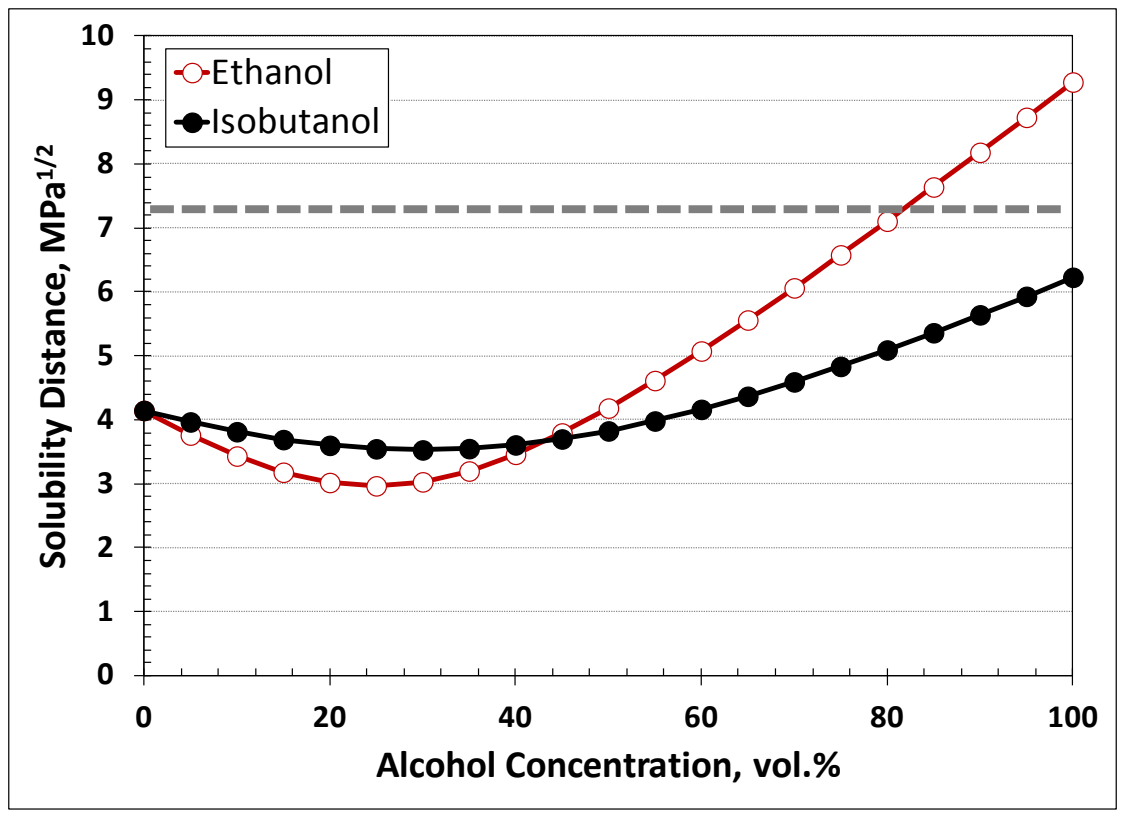

Fig. 10. Solubility distance curves for SBR for each alcohol type and concentration. The interaction radius for SBR is $7.3 \mathrm{MPa}^{1 / 2}$.

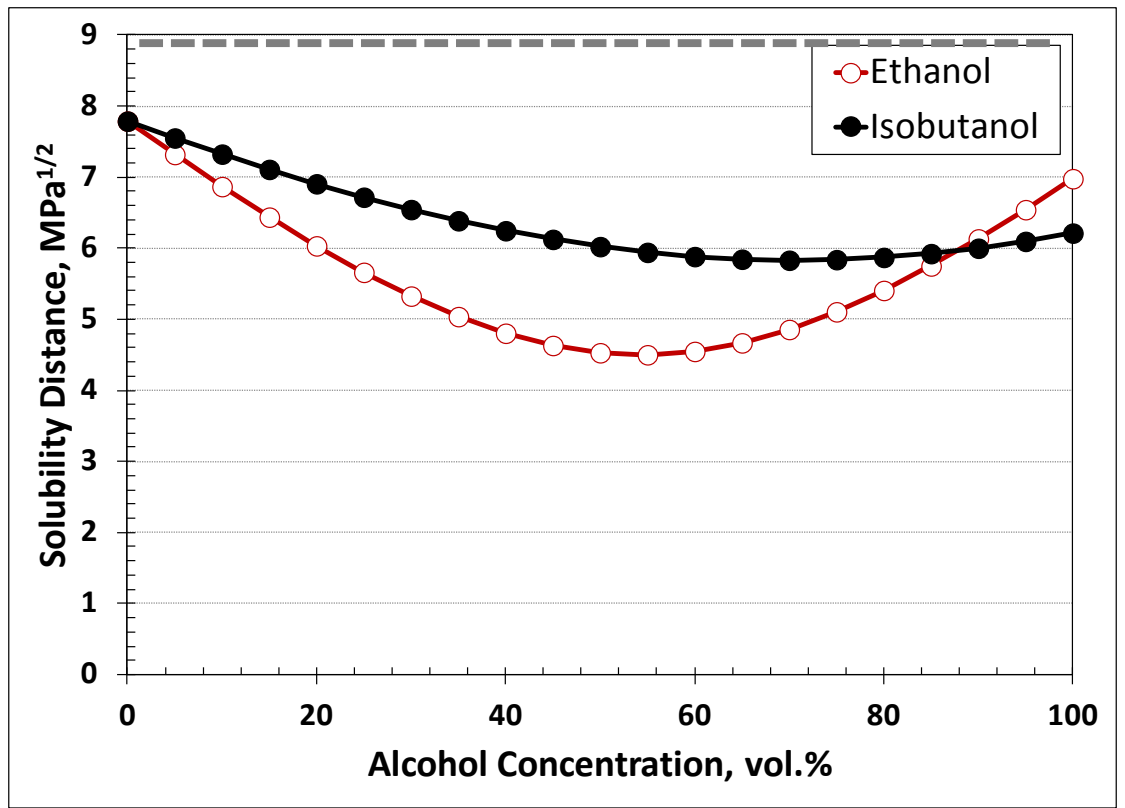

Fig. 11. Solubility distance curves for neoprene for each alcohol type and concentration. The interaction radius for neoprene is $8.9 \mathrm{MPa}^{1 / 2}$. 


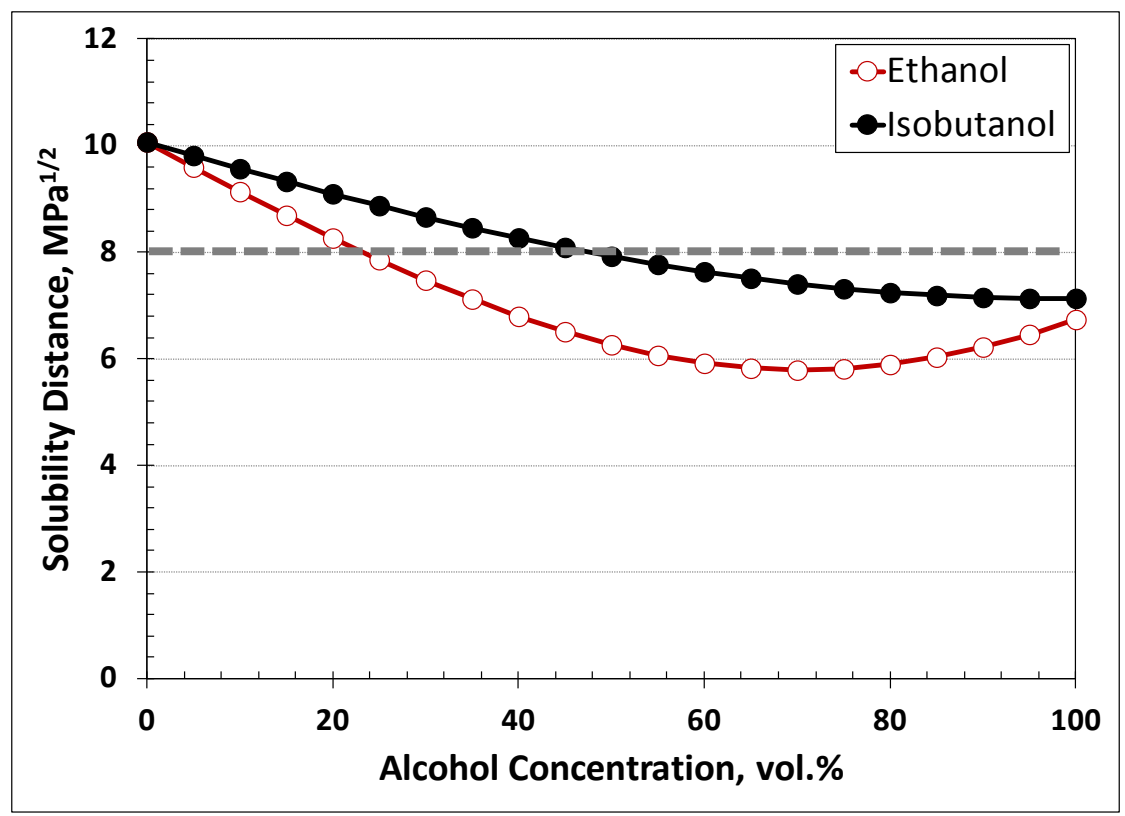

Fig. 12. Solubility distance curves for polyurethane for each alcohol type and concentration. The interaction radius for polyurethane is $8.0 \mathrm{MPa}^{1 / 2}$.

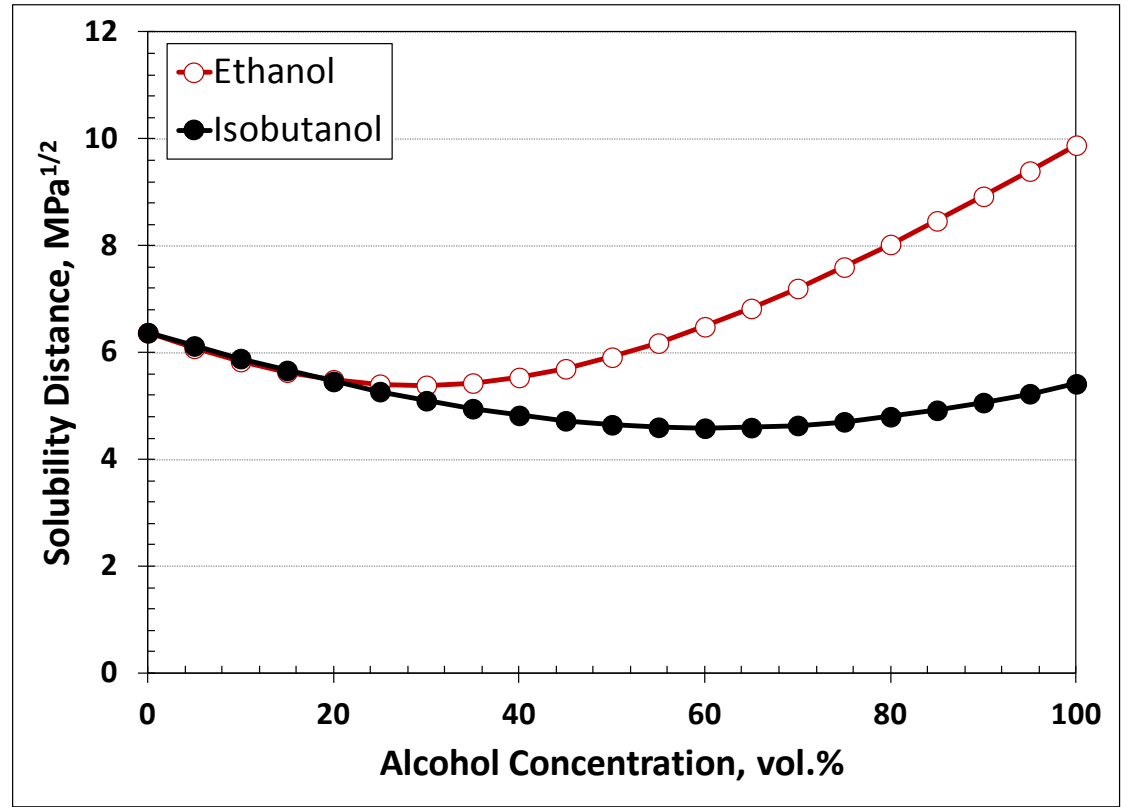

Fig. 13. Solubility distance curves for silicone rubber for each alcohol type and concentration. The interaction radius for silicone rubber is 14.3 $\mathrm{MPa}^{1 / 2}$. 
Since isobutanol will most likely be blended in gasoline at levels no higher than $24 \%$, the fuel blend compositions of most interest are those in which the isobutanol content is less than $50 \%$. For alcohol concentrations less than $20 \%$, the solubility distances for all elastomer materials generally were lower for (and therefore more soluble with) ethanol additions than isobutanol. (Silicone was the notable exception.) However, for very high alcohol concentrations, many of the elastomer materials yielded smaller $\mathrm{d}_{\mathrm{S}}$ values for isobutanol and exhibited increased solubility with higher isobutanol concentrations. (The one exception was polyurethane, which indicated reduced solubility in isobutanol over the entire range of composition.) The general implication is that isobutanol should have better compatibility with elastomers than ethanol at low concentrations, but at high concentrations many elastomers may be more compatible with ethanol. When the solubility curves are examined from an oxygen-equivalent standpoint, the solubility differences observed for isobutanol and ethanol become less pronounced. For many elastomers, these differences become negligible. For example, for fluoroelastomers and polyurethanes, the solubility distances for E10 and iBu16 are essentially the same.

When exposed to gasoline containing low alcohol levels, fluoroelastomers produced slightly higher $\mathrm{d}_{\mathrm{S}}$ values with isobutanol (as shown in Fig. 8) for equivalent alcohol concentrations. However, when viewed according to equivalent oxygen contents, the differences in $\mathrm{d}_{\mathrm{s}}$ (and predicted solubility) between the two fuels are negligible. Also, since the interaction radius for fluoroelastomer is higher than the $d_{S}$ values at low alcohol concentrations, a low level of solubility is predicted for alcohol concentrations less than $30 \%$.

The results for NBR (Fig. 9) and SBR (Fig. 10) were similar to each other; each exhibited only slightly better compatibility with isobutanol at concentrations less than $50 \%$, with peak swelling (lowest $\mathrm{d}_{\mathrm{S}}$ value) occurring around $25 \%$ for each. For both of these elastomer types, the predicted swelling is lower for the pure alcohols when compared to Fuel C. The interaction radii for both NBR and SBR are much higher than the solubility distances for low alcohol concentrations for both materials, so moderate to high solubility (or swelling) is expected over this range.

Over most of the compositional range, the $d_{S}$ values for neoprene (Fig. 11) were lower for ethanol, indicating that a small increase in swelling would be expected with ethanol (compared to isobutanol). The interaction radius for neoprene is $8.9 \mathrm{MPa}^{1 / 2}$, which is slightly higher that the $d_{\mathrm{S}}$ values at low alcohol concentrations. The implication is that low to moderate swelling is predicted for this material at alcohol concentrations less than $25 \%$.

The solubility curves for polyurethane are shown in Fig. 12. The curves are somewhat similar to those for neoprene, except that the $d_{S}$ values were lower for ethanol over the full compositional range. However, in contrast to the other elastomer materials, the interaction radius for polyurethane $\left(8.0 \mathrm{MPa}^{1 / 2}\right)$ is significantly lower than the $d_{S}$ values derived for alcohol concentrations less than $25 \%$. The implication is that polyurethane will have low solubility to the fuels tested in this study (CE10a, CE17a, CiBu16a, and CiBu24a).

Silicone (Fig. 13) showed no difference in solubility for either alcohol type when the concentrations were less than 20\%. However, higher alcohol levels resulted in $\mathrm{d}_{\mathrm{s}}$ being higher for ethanol (than isobutanol), and as alcohol concentration is increased, this difference in $\mathrm{d}_{\mathrm{s}}$ becomes more pronounced. Previous efforts have shown that silicone does not swell significantly with high ethanol concentrations, but the implication here is that isobutanol (at high levels) will cause silicone to swell noticeably and perhaps even exceed the swelling levels observed at lower concentrations. When viewed on an oxygen equivalent basis, the $d_{S}$ values for ethanol are actually slightly higher than for isobutanol at low alcohol concentrations, but because the values are so similar, no difference in swelling behavior is expected for either alcohol type. It is important to note that the interaction radius for silicone is substantially higher than the resulting $\mathrm{d}_{\mathrm{S}}$ values, especially for low alcohol content; therefore, high solubility (and hence swelling) is expected for exposure to the test fuels (CE10a, CE17a, CiBu16a, and CiBu24a). 


\subsection{PLASTICS}

The plastic materials are grouped and discussed in the following four categories:

- permeation barriers,

- nylons and HDPE,

- fiberglass resins and epoxies, and

- other common plastics.

As stated previously, the permeation barrier materials, nylons and HDPE, and other common plastics are thermoplastic materials. The polyester resins and epoxies are thermosets. The permeation barrier materials included in this study along with the nylons, HDPE, and the fiberglass resins are commonly used in legacy and new fuel storage and dispensing systems. Permeation barrier materials typically have the best compatibility properties and are used in applications requiring direct contact with the fuels and/or their vapors to prevent and minimize fuel leakage. Nylons and HDPE are used primarily as structural reinforcement for flexible plastic piping and in components, such as valves, pumps, etc., and are not necessarily designed for long-term direct exposure to the fuels. The fiberglass resins are used as matrix materials in rigid fiberglass piping and underground storage tanks, and they also come into direct contact with fuel liquids and vapors. Epoxies are included with the fiberglass resins since they are all thermosetting plastic materials and, therefore, have similar properties. The remaining plastic materials, while not directly identified with a specific fuel storage component or system, are widespread and commonly used in many applications.

\subsubsection{Permeation Barrier Materials}

These materials are used as permeation barriers in piping and storage systems and, therefore, are expected to have excellent compatibility to gasoline-type fuels. The four barrier materials evaluated in this study included PPS, PTFE, PVDF, and PET, and their respective solubility to gasoline containing ethanol and isobutanol are shown in Figs. 14-17. A common feature of these four materials is that the interaction radii are significantly lower than their corresponding solubility distances in gasoline containing either ethanol or isobutanol; this is especially true for low alcohol concentrations. Comparison of the polymer interaction radii with the solubility distance curves suggests that PPS, PVDF, and PET would be insoluble to the test fuel formulations. PTFE, on the other hand, would be expected to exhibit moderate solubility for low alcohol concentrations, since its interaction radius $\left(3.9 \mathrm{MPa}^{1 / 2}\right)$ is higher than $\mathrm{d}_{\mathrm{S}}$ for isobutanol concentrations less than $55 \%$ and ethanol concentrations below $85 \%$.

Of the four barrier materials, PET (or Mylar) is most commonly used due to its lower relative cost. The most expensive of these materials is PPS, which is less utilized as a piping barrier. As shown in Figs. 1417 , these plastics all exhibited higher $d_{S}$ values for isobutanol compared to ethanol for low alcohol levels. In each case the solubility distance decreased with increasing alcohol content, and for PPS and PET, $\mathrm{d}_{\mathrm{s}}$ reaches a minimum value at concentrations near $60 \%$. For PTFE, the location of minimum $\mathrm{d}_{\mathrm{S}}$ values occurred around 25\% ethanol and 30\% isobutanol. In contrast, the solubility distance for PVDF decreased linearly with alcohol concentration, an indication that PVDF may be susceptible to pronounced swelling at high alcohol concentrations. PTFE behaved differently from the other barrier materials in that $d_{\mathrm{s}}$ values were lowest at low alcohol concentrations and highest for the pure alcohols, an indication that PTFE is much less soluble to alcohols than Fuel C. 


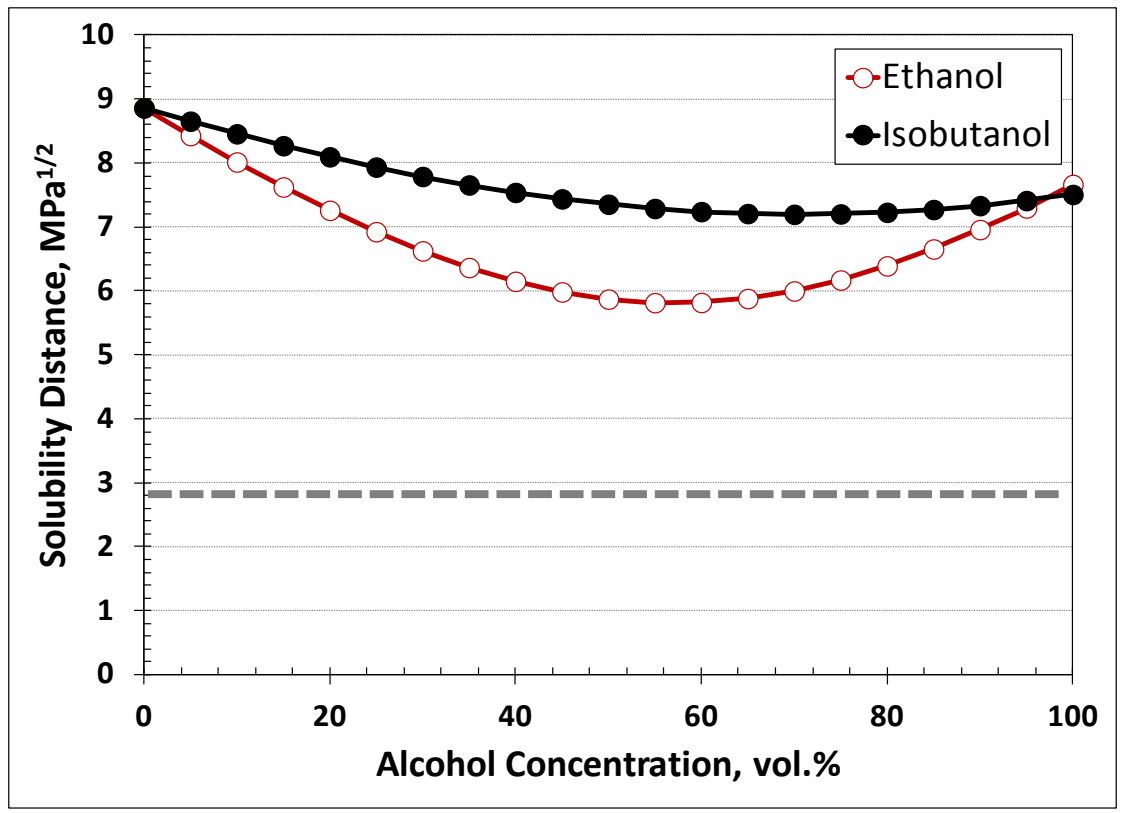

Fig. 14. Solubility distance curves for PPS for each alcohol type and concentration. The interaction radius for PPS is $2.8 \mathrm{MPa}^{1 / 2}$.

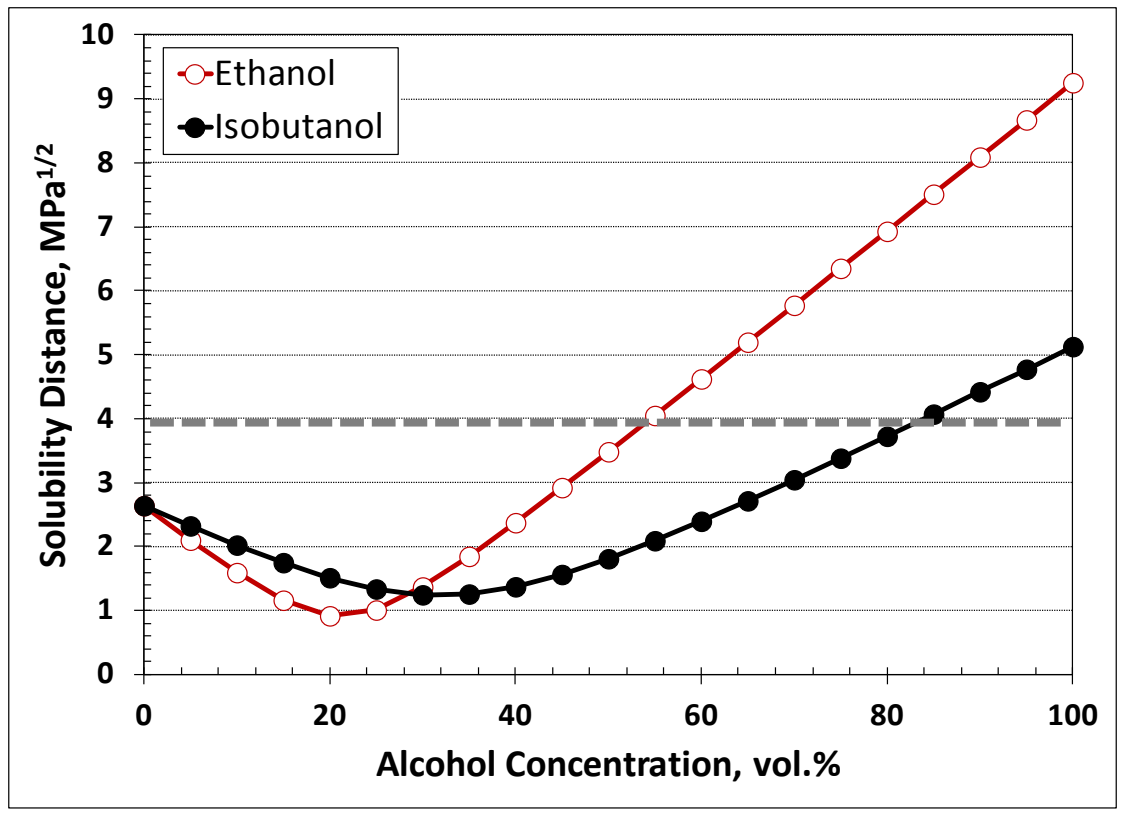

Fig. 15. Solubility distance curves for PTFE for each alcohol type and concentration. The interaction radius for PTFE is $3.9 \mathrm{MPa}^{1 / 2}$. 


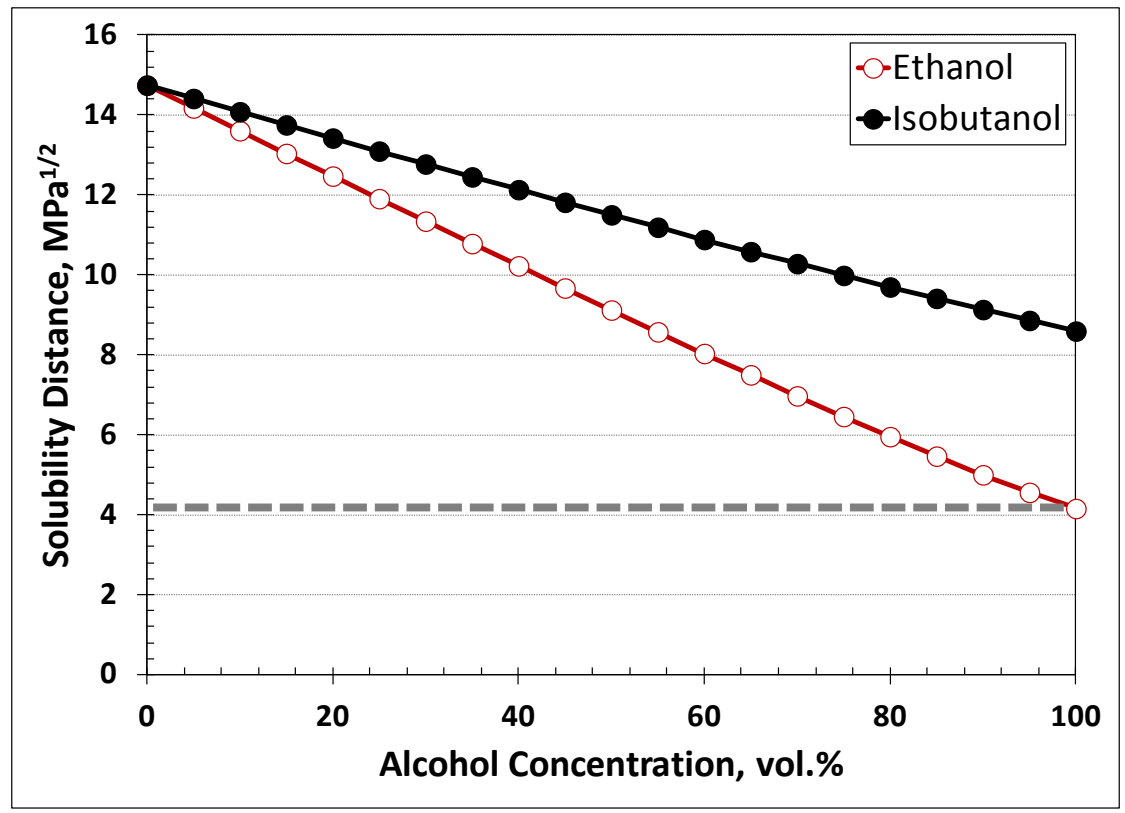

Fig. 16. Solubility distance curves for PVDF for each alcohol type and concentration. The interaction radius for PVDF is $4.1 \mathrm{MPa}^{1 / 2}$.

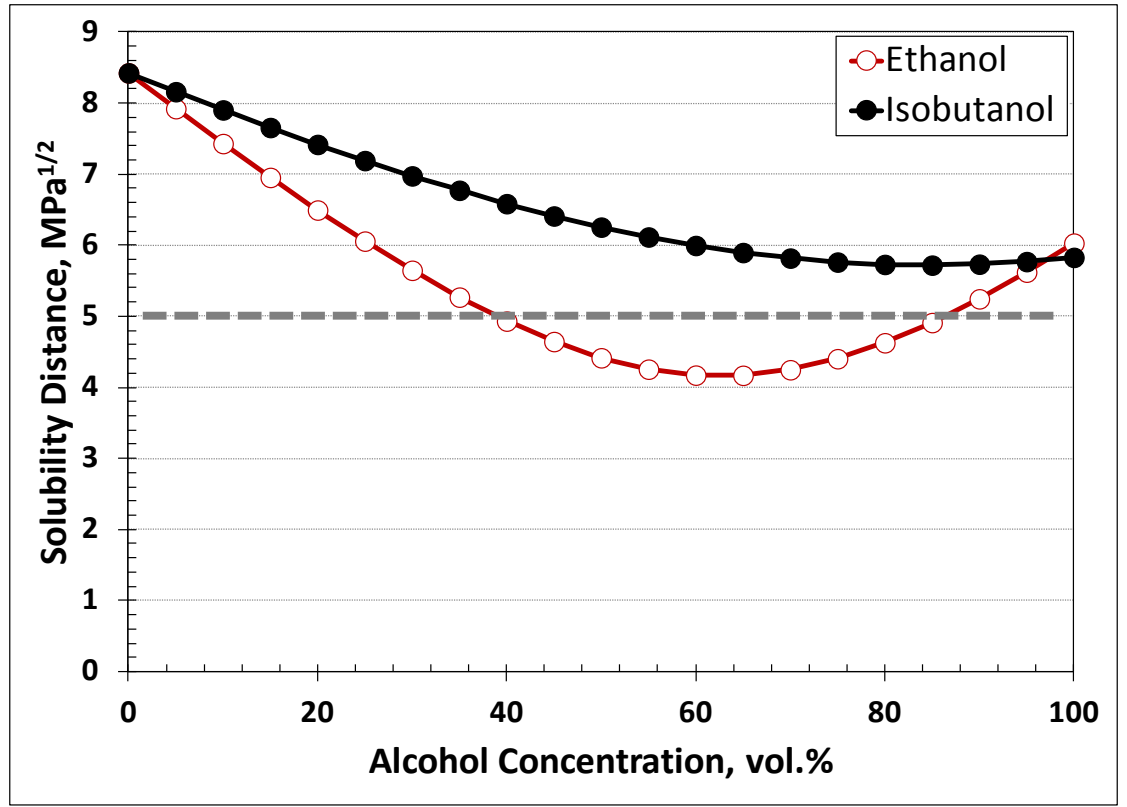

Fig. 17. Solubility distance curves for PET for each alcohol type and concentration. The interaction radius for PET is $5.0 \mathrm{MPa}^{1 / 2}$. 


\subsubsection{Nylons and HDPE}

The four nylons evaluated in this study were Nylon 6, Nylon 6,6, Nylon 12, and Nylon 11. HDPE was also included in this grouping since it (along with the nylons) is also used as a wall material in plastic piping systems. The curves for the nylons are shown in Figs. 18-21 and those for HDPE are shown in Fig. 22. The interaction radius for each material is listed in each figure caption, and for these materials $I_{R}$ is significantly lower than $\mathrm{d}_{\mathrm{s}}$, indicating insolubility with the test fuel formulations.

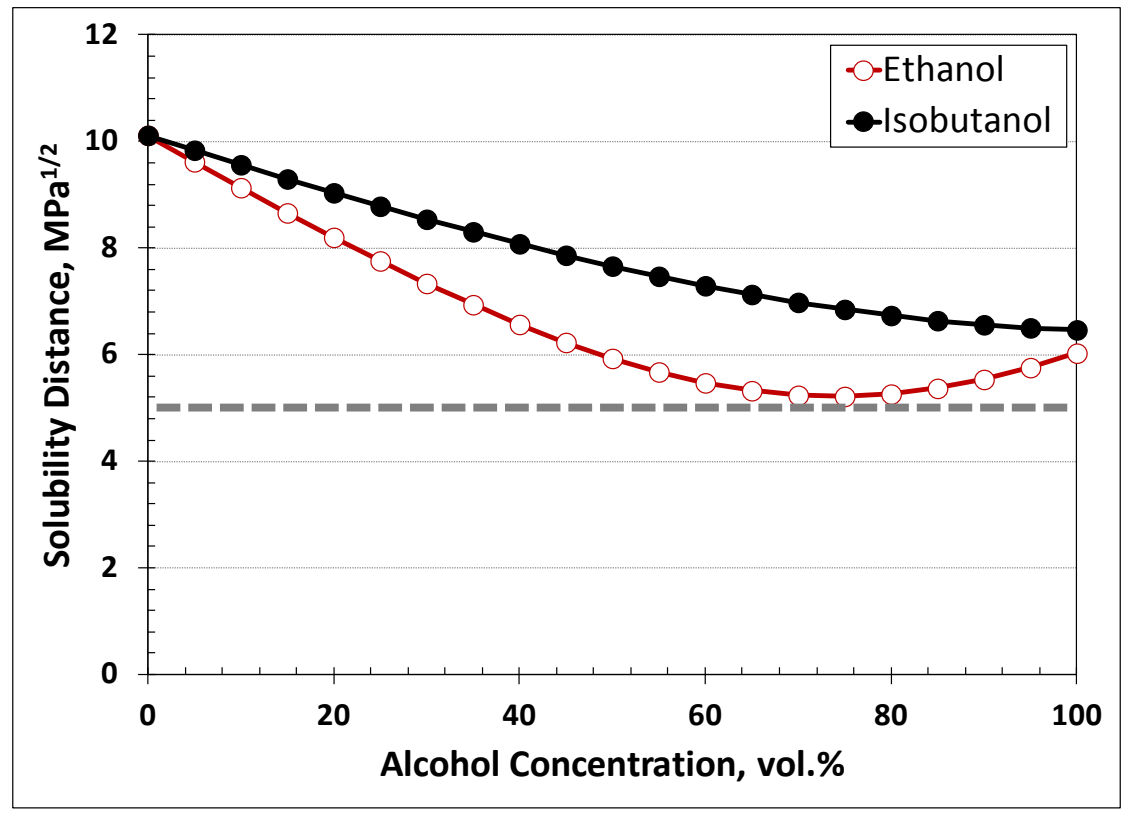

Fig. 18. Solubility distance curves for Nylon 6 for each alcohol type and concentration. The interaction radius for Nylon 6 is $5.1 \mathrm{MPa}^{1 / 2}$.

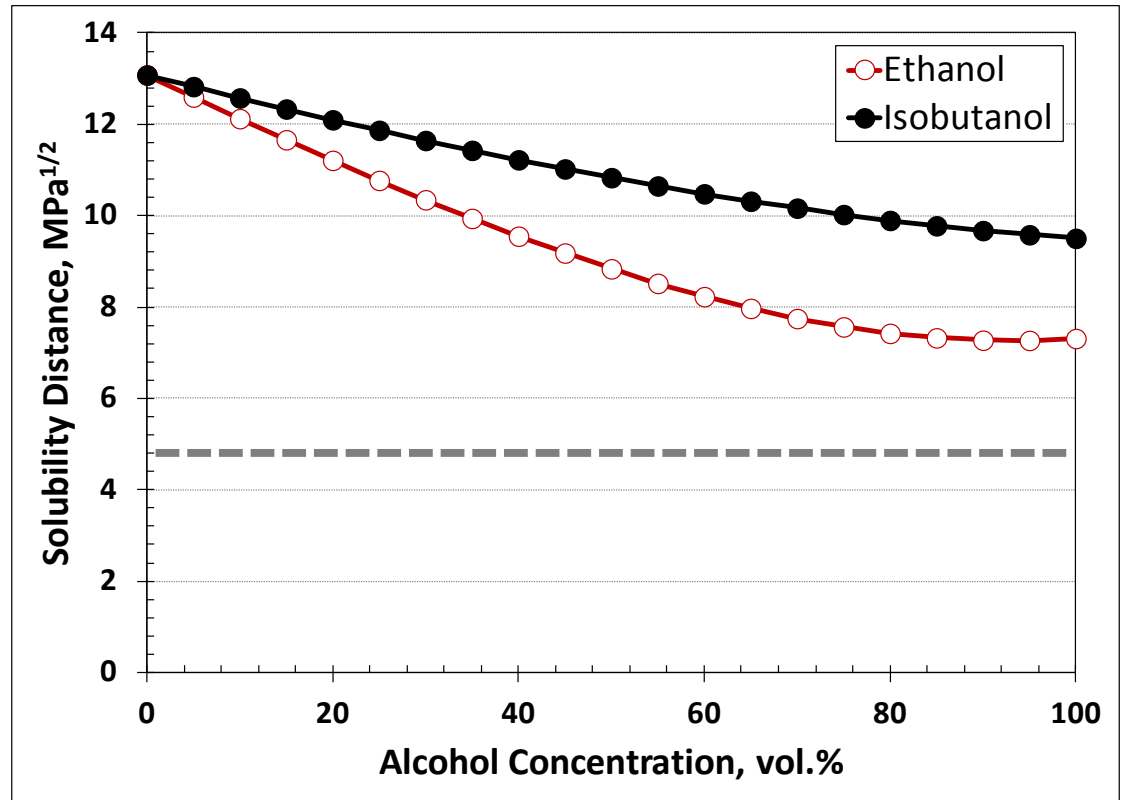

Fig. 19. Solubility distance curves for Nylon 6,6 for each alcohol type and concentration. The interaction radius for Nylon 6,6 is $4.4 \mathrm{MPa}^{1 / 2}$. 


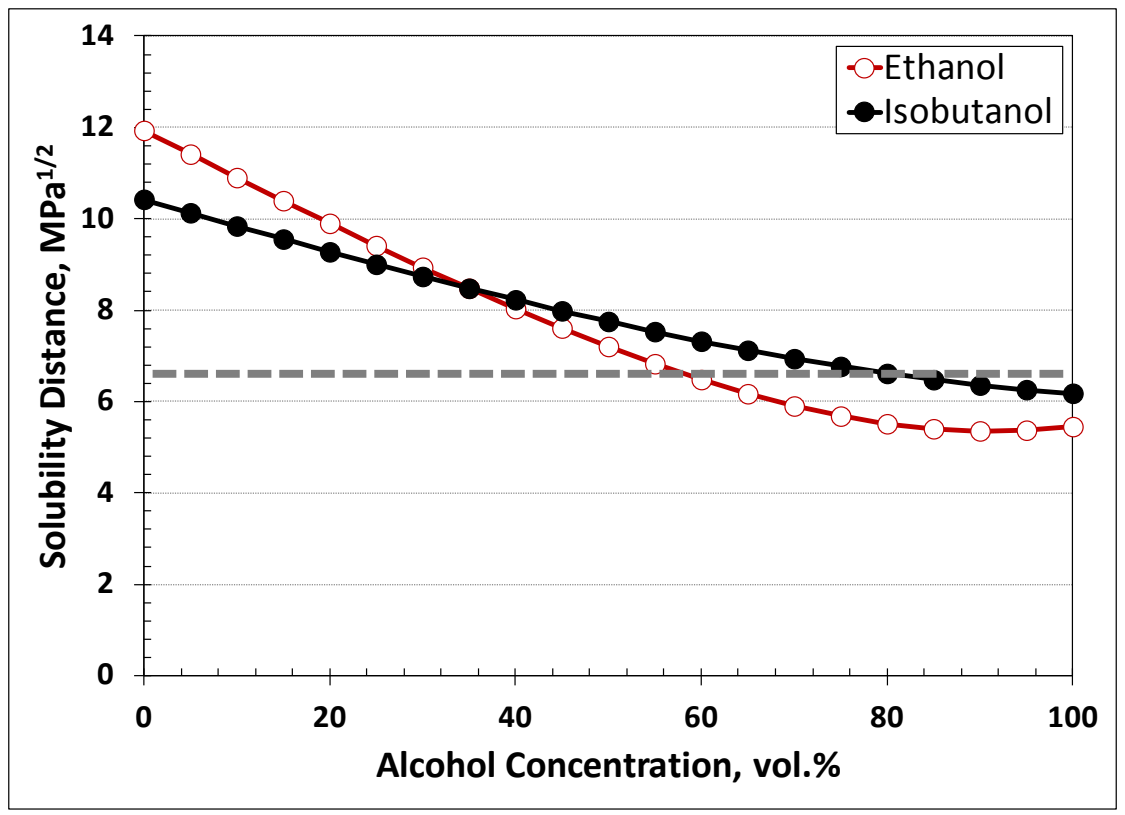

Fig. 20. Solubility distance curves for Nylon 12 for each alcohol type and concentration. The interaction radius for Nylon 12 is $6.3 \mathrm{MPa}^{1 / 2}$.

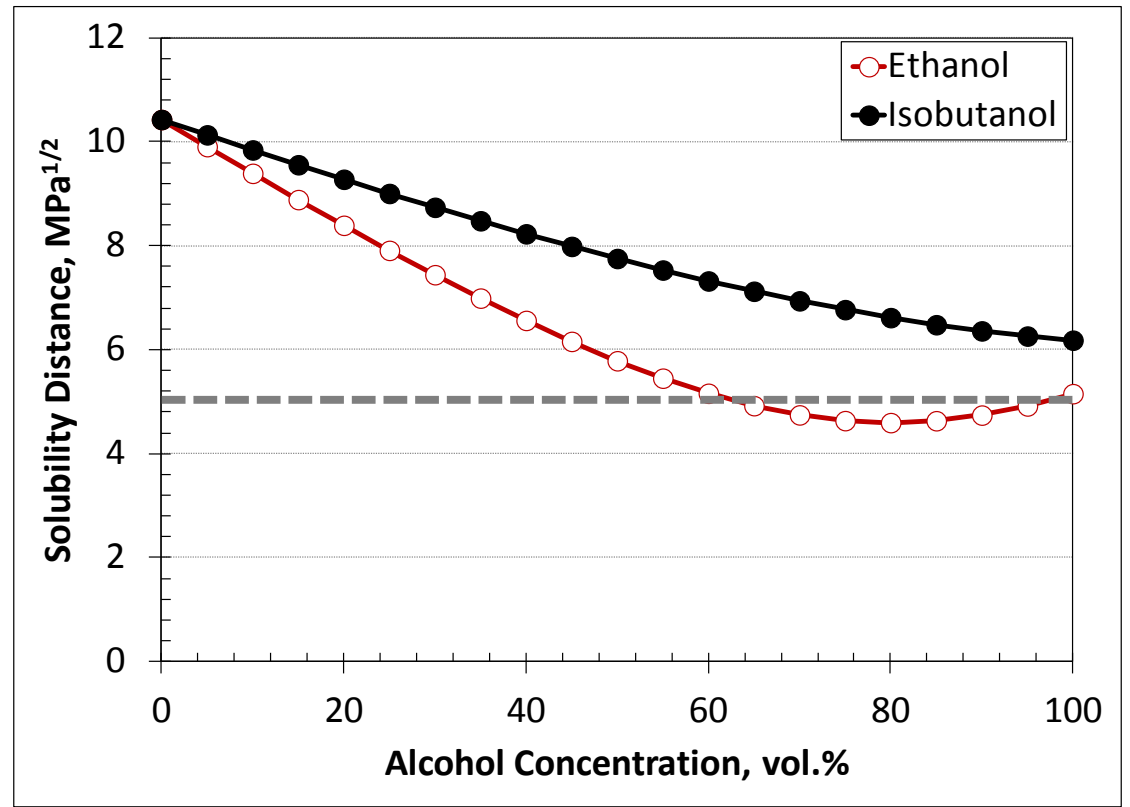

Fig. 21. Solubility distance curves for Nylon 11 for each alcohol type and concentration. The interaction radius for nylon 11 is $5.1 \mathrm{MPa}^{1 / 2}$. 


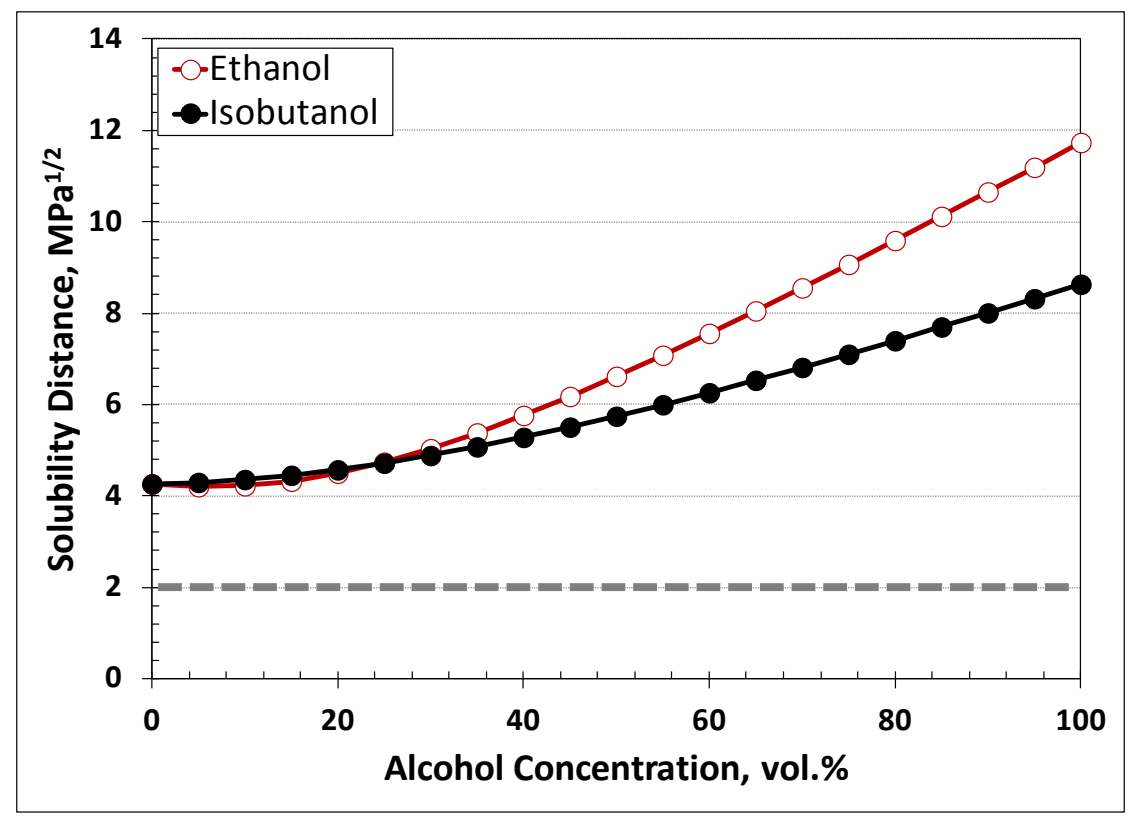

Fig. 22. Solubility distance curves for HDPE for each alcohol type and concentration. The interaction radius for $\mathrm{HDPE}$ is $2.0 \mathrm{MPa}^{1 / 2}$.

Further analysis shows that the solubility behavior for the nylons was observed to differ according to alcohol type. The solubility distance decreased with increasing isobutanol concentration over the entire range of composition. Ethanol also tended to reduce the solubility distance with increased concentration; however, a clear minimum value was achieved for concentrations between 70 and $95 \%$. The resulting curves for Nylon 6, Nylon 6,6, and Nylon 11 were similar, and over the full range of composition, $\mathrm{d}_{\mathrm{S}}$ values were lower for ethanol than isobutanol, indicating better compatibility with isobutanol. Nylon 12 was different in that the $\mathrm{d}_{\mathrm{s}}$ values for concentrations less than $35 \%$ were lower for isobutanol than ethanol, an indication that Nylon 12 may actually be less compatible to isobutanol than ethanol at low concentrations. However, it is important to note that the differences in $\mathrm{d}_{\mathrm{S}}$ for the two alcohol types are relatively small and likely not significant.

As can be seen in Fig. 22, HDPE differed from the other plastics in that the solubility distance was observed to increase with increasing alcohol concentration. This result contrasts with the other plastics whereby $\mathrm{d}_{\mathrm{S}}$ decreased with increasing alcohol content. Another contrasting characteristic is that for concentrations less than $35 \%$, there is no significant difference in $\mathrm{d}_{\mathrm{s}}$ for either ethanol or isobutanol. The implication is that HDPE will exhibit the highest level of swelling with Fuel C exposure and the least with neat alcohol exposure.

\subsubsection{Fiberglass Resins and Epoxies}

Four resin materials were evaluated in this study. Two of them were isophthalic resins used in legacy fiberglass tanks, and the other two included a current terephthalic polyester resin and a newer vinyl ester resin. The literature survey showed identical HSPs for both terephthalic and isophthalic esters, and the resulting solubility curve is shown in Fig. $23 .{ }^{17}$ The solubility curve for the vinyl ester is shown in Fig. 24 and is similar to the other resin types. For all resins, the solubility distance was observed to decrease with increasing alcohol content over the entire compositional range. Therefore, solubility for these resins can be expected to increase with increasing alcohol content. The solubility curves for both isophthalic and terephthalic polyester resins (Fig. 23) suggest that these polyesters will exhibit low to moderate solubility to the test fuel formulations. The vinyl ester resin can be expected to behave similarly, but for low alcohol 
concentrations (representative of the test fuel formulations) as shown in Fig. 24. These curves suggest negligible to low swelling since the interaction radius is lower than $\mathrm{d}_{\mathrm{s}}$. For all resin materials, the actual difference in $\mathrm{d}_{\mathrm{S}}$ between the alcohols is small for low concentrations, indicating that isobutanol may offer only marginally improved performance compared to ethanol.

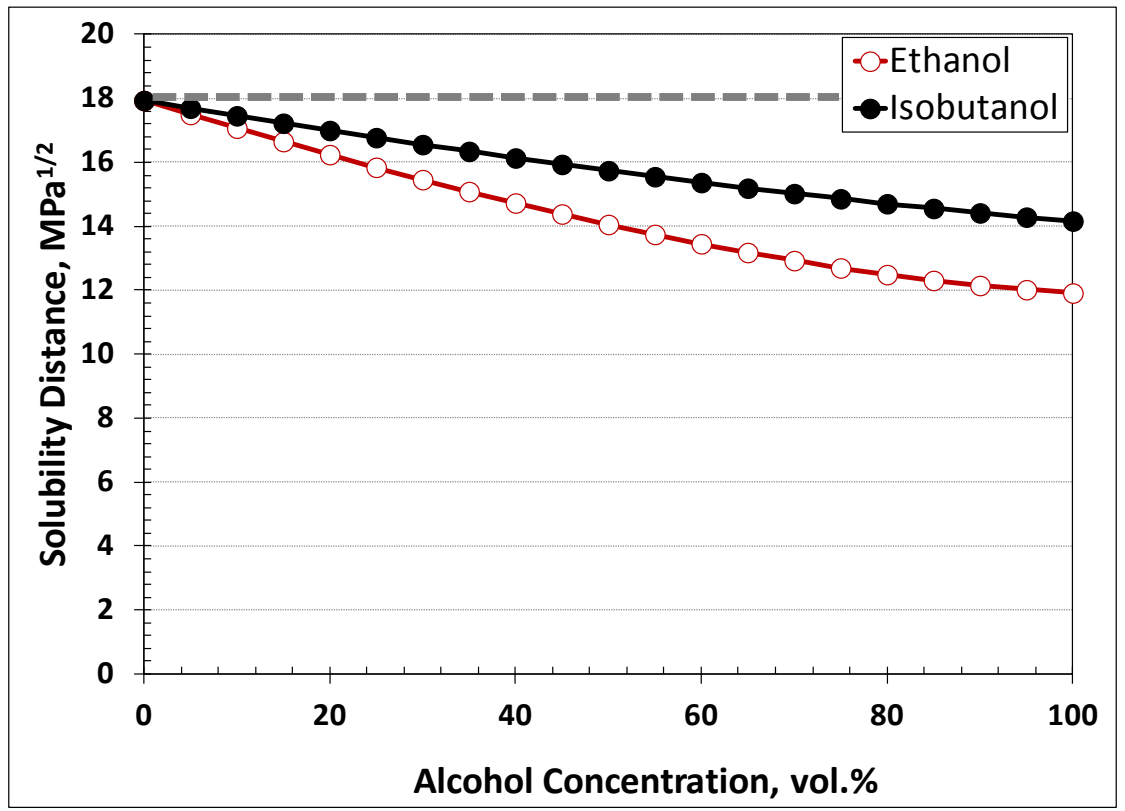

Fig. 23. Solubility distance curves for terephthalic and isophthalic polyester resins for each alcohol type and concentration. The interaction radius for these resins is $18.0 \mathrm{MPa}^{1 / 2}$.

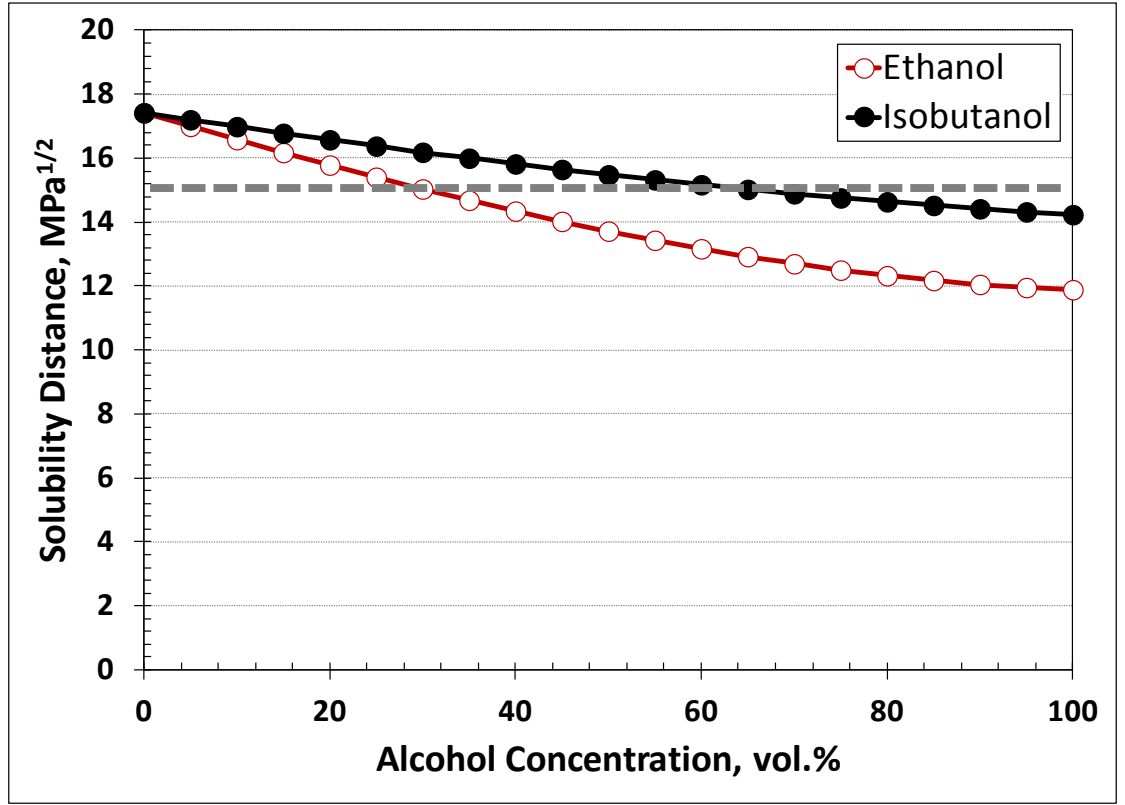

Fig. 24. Solubility distance curves for vinyl ester resins for each alcohol type and concentration. The interaction radius for vinyl ester is $15.1 \mathrm{MPa}^{1 / 2}$. 
The solubility curves for epoxy resins are shown in Fig. 25 for two curing conditions. Cold cure took place at room (or ambient) temperatures, while hot curing occurred at $100^{\circ} \mathrm{C}$. The solubility distance decreases sharply with increasing alcohol concentration over the entire range for each alcohol and each curing condition. For hot-cured conditions the solubility distances are significantly higher than for a cold cure, indicating that epoxies that are hot cured will exhibit improved compatibility with alcohol fuels. For gasoline blended with isobutanol, the solubility distances are higher than for ethanol, and this gap between the two alcohol types increases with fuel alcohol content. Interestingly, the interaction radii for both curing conditions is significantly less than the solubility distances for fuels containing low levels of alcohol, indicating that low to moderate solubility can be expected for the test fuel formulations.

However, alcohol concentrations exceeding $50 \%$ can be expected to be highly soluble with epoxies.

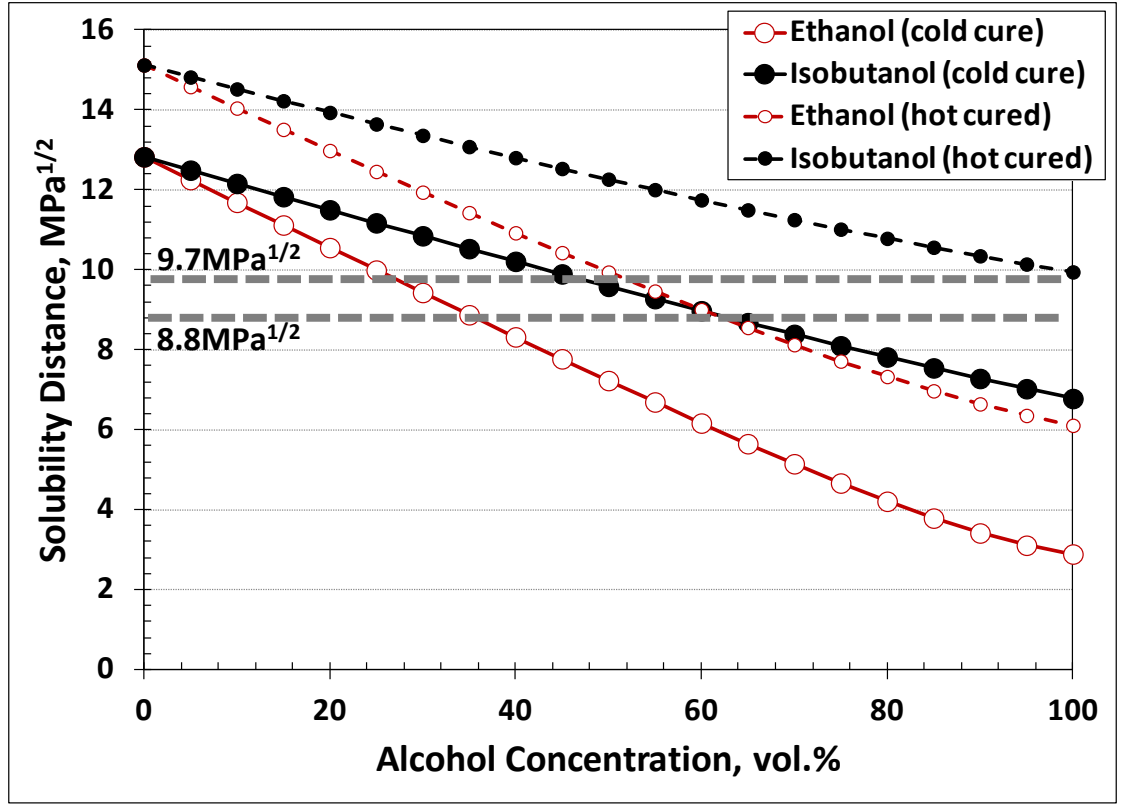

Fig. 25. Solubility distance curves for epoxy resins for each alcohol type and concentration. Two curing processes (hot and cold) are included for comparison. The interaction radii for cold and hot cures are 8.8 and 9.7 $\mathrm{MPa}^{1 / 2}$, respectively.

\subsubsection{Other Common Plastics}

Other common plastics include well-known mid-range and commodity types not yet identified in fueling infrastructure, yet their widespread use indicates that they may be used in some applications. Typically these materials are of low to moderate cost. They include PBT, PETG, POM, PP, and PTU. However, HSPs could not be found for PTU, so it was not included in this analysis.

PBT and PETG polyesters were included in this grouping. PETG is actually a copolymer of PET, and the addition of a second component greatly changes the behavior as shown in Fig. 26. The curves indicate higher solubility of PETG in gasoline containing ethanol rather than isobutanol. The results also suggest that PETG will be more soluble (than PET) for isobutanol concentrations less than $50 \%$, but higher concentrations indicate more solubility in PET, relative to PETG. 

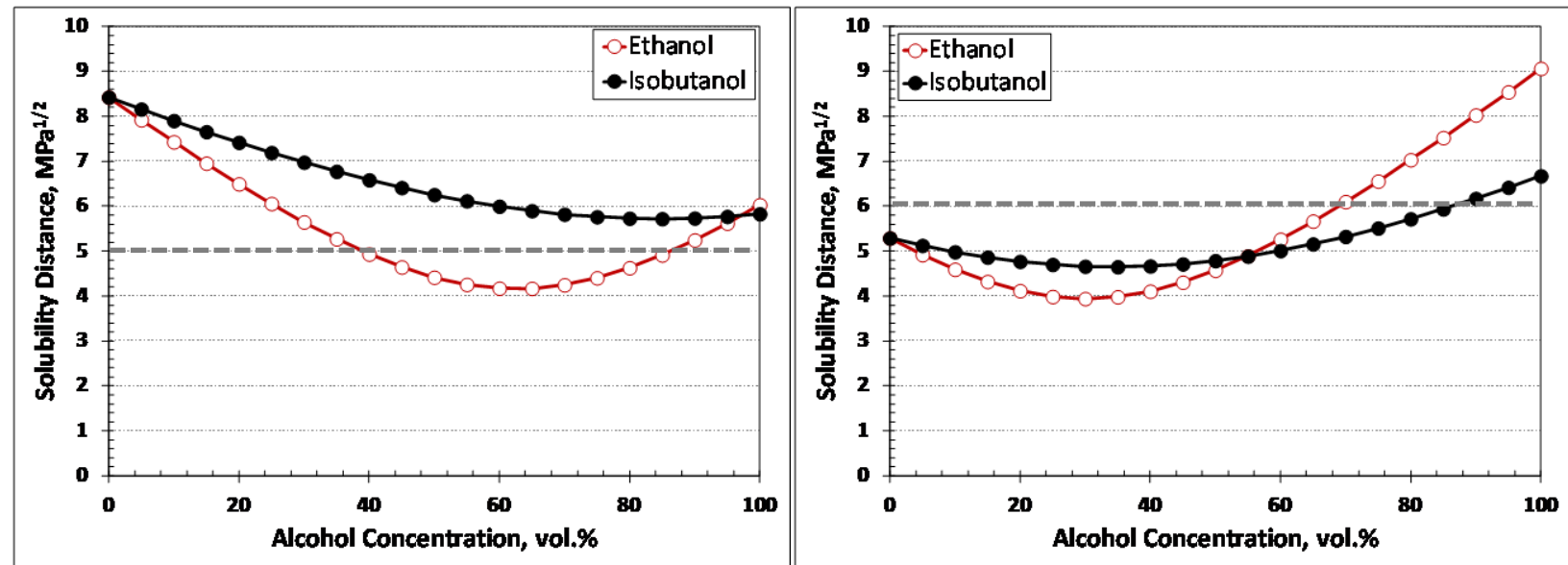

Fig. 26. Solubility distance curves for PET and PETG for each alcohol type and concentration. The interaction radius for PETG is 6.0 $\mathrm{MPa}^{1 / 2}$.

The solubility curves for PBT (shown in Fig. 27) closely match those obtained for PET (Fig. 17). As a result, the swelling behavior for PBT in gasoline containing ethanol or isobutanol should approximate that for PET. As with PET, PBT exhibits lower $d_{s}$ values than ethanol for the full range of composition, and therefore PBT would be expected to exhibit less volume swell with isobutanol compared to ethanol. On an oxygen equivalent basis, however, the solubility difference between the two alcohols is slight, and negligible differences may be expected.

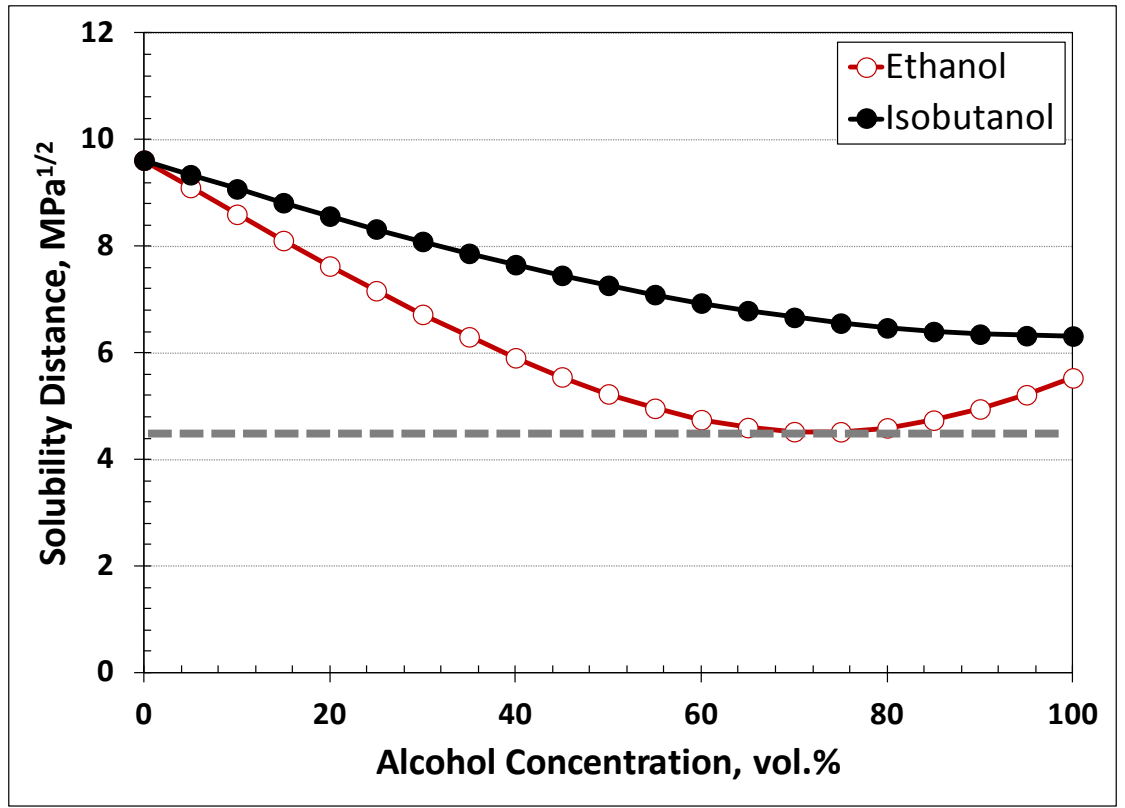

Fig. 27. Solubility distance curves for PBT for each alcohol type and concentration. The interaction radius for $\mathrm{PBT}$ is $4.5 \mathrm{MPa}^{1 / 2}$.

The solubility curves for POM (acetal) and PP are shown in Figs. 28 and 29, respectively. The results indicate that the solubility of POM sharply increases with alcohol content over the entire range. POM can be expected to exhibit higher solubility with ethanol, and the relative increase in this solubility (compared 
to isobutanol) is larger as the alcohol concentration approaches $100 \%$. However, for low alcohol concentrations, the solubility difference between isobutanol and ethanol is expected to be low.

The predicted solubility behavior for PP (Fig. 29) is similar to that of HDPE in that the solubility distance increases with alcohol content over the entire range of composition. These were the only two plastic types in which $\mathrm{d}_{\mathrm{S}}$ increased with increased alcohol concentration. Additionally, both PP and HDPE exhibited lower $d_{S}$ values with isobutanol, an indication these materials would experience higher swelling in isobutanol.

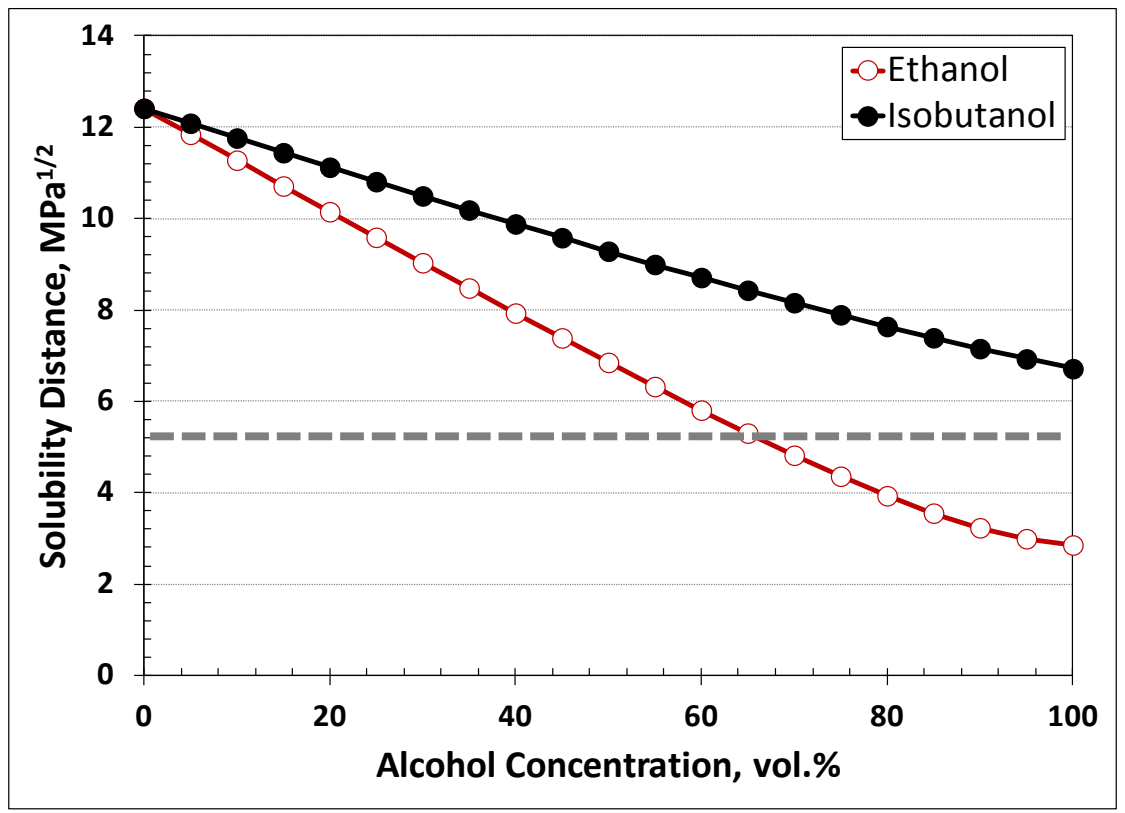

Fig. 28. Solubility distance curves for POM for each alcohol type and concentration. The interaction radius for POM is $5.3 \mathrm{MPa}^{1 / 2}$.

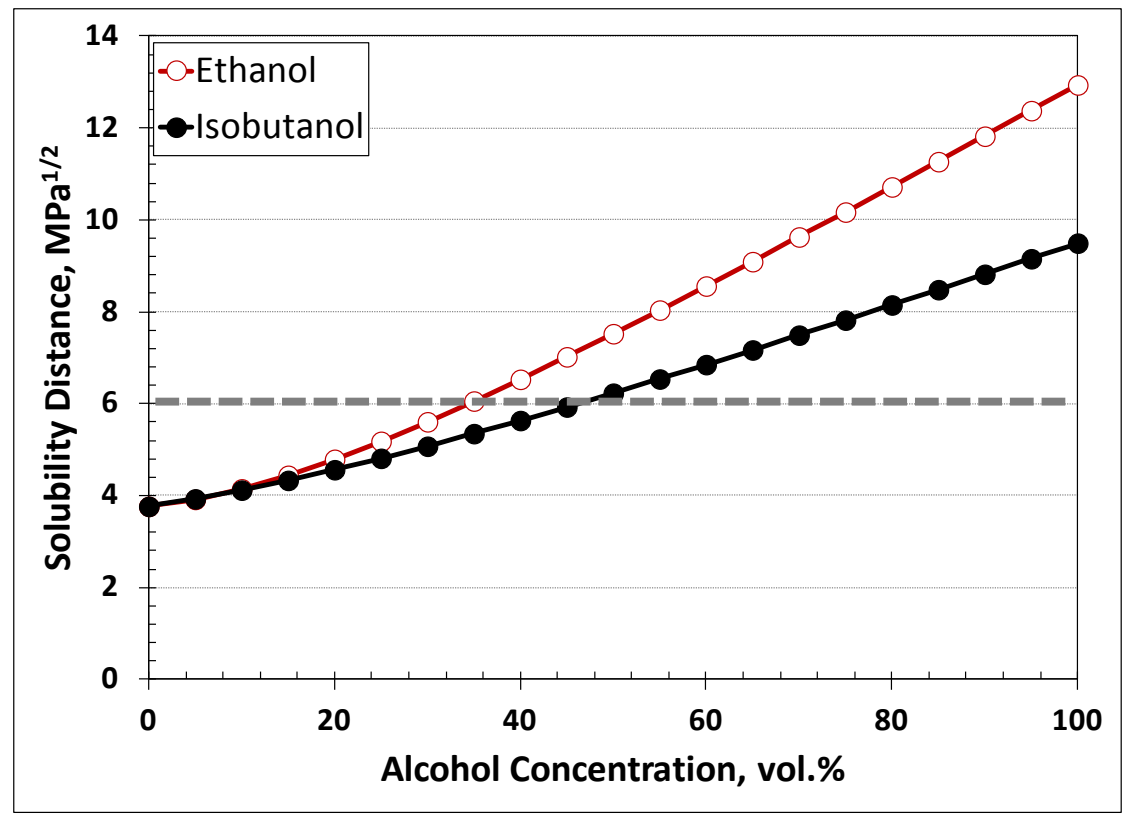

Fig. 29. Solubility distance curves for $P P$ for each alcohol type and concentration. The interaction radius for $P P$ is $6.0 \mathrm{MPa}^{1 / 2}$. 



\section{EXPERIMENTAL ANALYSES}

The experimental results for the polymers are presented as bar charts, while the mass loss results and surface chemistries for the metal materials are presented in tables and depth profile plots, respectively. For the polymers, the mass and volume results represent the average of three values (one for each specimen tested). The hardness measurements were made at five locations on each specimen (one in the center and four near the corners). Since three specimens were exposed to the liquids and two to the vapors, the total number of hardness measurements for liquid and vapor exposures was 15 and 10, respectively. In addition to presenting the mean values for each property measured, the standard error of the mean was included as error bars for each result. DMA was performed on only one specimen for each material type, so no error range is given.

\subsection{ELASTOMERS}

The elastomer specimens (exposed to the test fuel liquid and vapor phases) were removed from the environmental chambers after 4 weeks of exposure. The wetted specimens were quickly transferred to a secondary container partially filled with test fuel to prevent drying, while the vapor exposures were placed inside a plastic bag. Upon removal from the chamber, the specimens exposed to the headspace vapors appeared to be dry, with no hint of condensation. The elastomer specimens that were immersed in the liquid test fuels were measured for mass, volume, and hardness, and these results were compared to the original pre-exposure values to obtain the change in these properties associated with exposure to the test fuel liquids. These specimens were also measured by DMA in order to determine the temperature associated with the onset of the glass-to-rubber transition. In contrast to the physical property measurements, the DMA apparatus required dry specimens, so measurements in the wetted state could not be determined.

\subsubsection{Wet Mass and Volume}

When exposed to the test fuels, elastomers tend to undergo a certain amount swelling or volume expansion that is proportional to the level of mutual solubility that exists between the elastomer and the solvent. The solvent is typically a liquid, but it can also be a vapor. The increases in both mass and volume for each elastomer type and test fuel composition are shown side by side in Fig. 30.

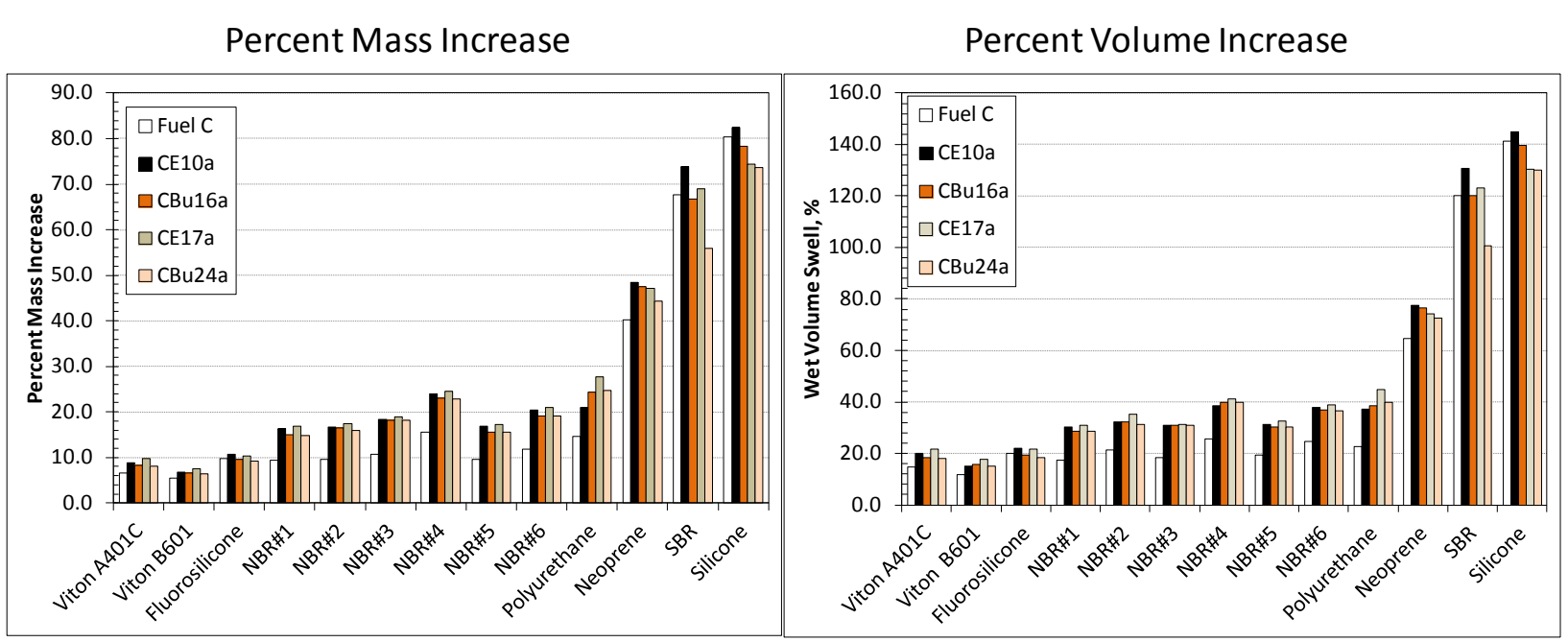

Fig. 30. Mass and volume increases for the elastomers exposed to the test fuel liquids for 4 weeks. 
The volume swell is highly proportional to the mass increase, and as shown in the figure, it is roughly twice as great as the increase in mass. The fluoroelastomers swelled the least, followed by the NBRs, polyurethane, and neoprene in extent of swell. The highest level of swelling occurred for the SBR and silicone rubber (which swelled the most).

\subsubsection{Fluoroelastomers}

The two fluorocarbons evaluated in this study were Viton A401C and Viton B601. Viton A401C is a common fluorocarbon that contains $66 \%$ fluorine. Viton B601 has a similar composition but contains a slightly higher (68\%) fluorine content. The increases in both mass and volume for these materials following exposure to the test fuels liquids are shown in Fig. 31 along with the results of the fluorosilicone rubber specimen. As predicted by the solubility curves, the overall extent of swelling after contact with the test fuels was relatively low.

Percent Mass Increase

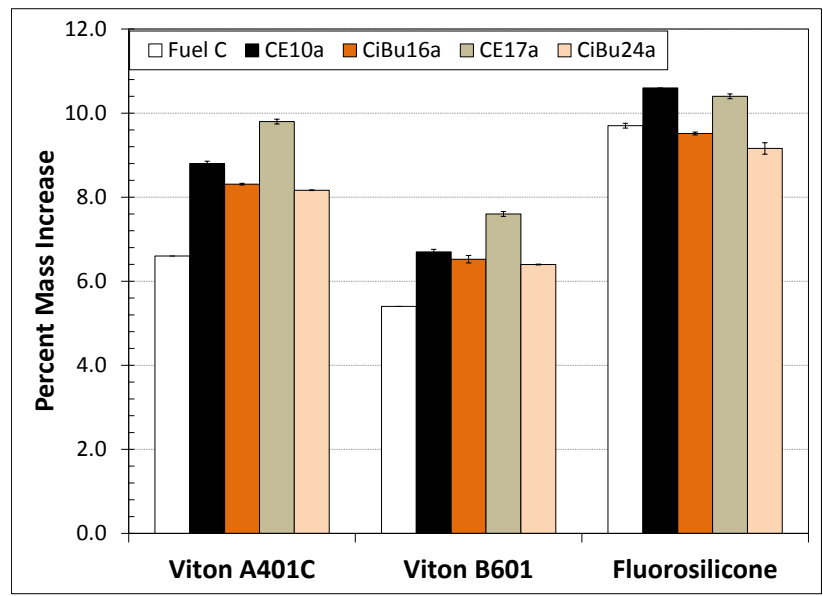

Percent Volume Increase

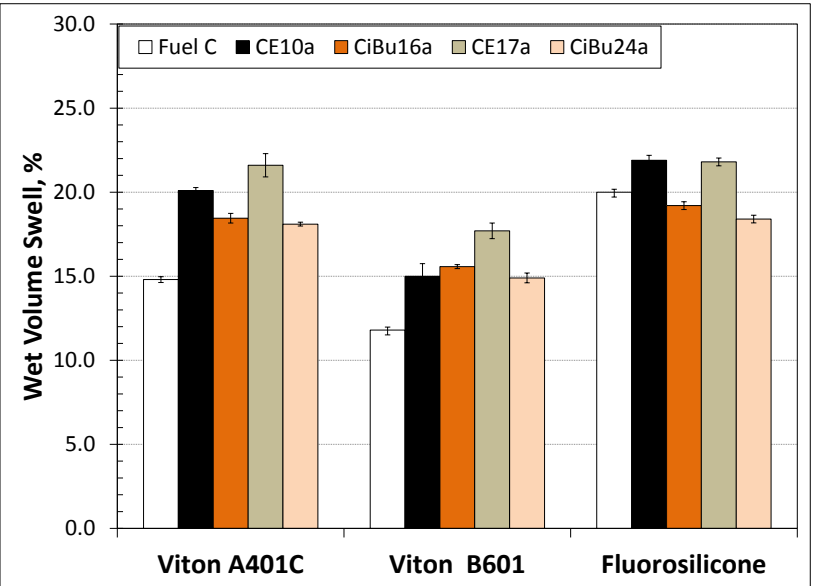

Fig. 31. Mass and volume increases for the fluoroelastomers exposed to the test fuel liquids for 4 weeks.

In general the observed volume expansion is roughly twice the mass increase for the fluoroelastomer specimens. In all cases the relative increase in mass was accompanied by a corresponding proportional increase in volume. For the fluoroelastomers, the level of swell was slightly lower for fuels containing isobutanol than those having ethanol. This result follows that predicted by the solubility analysis in Fig. 8 . All of the fluoroelastomer specimens swelled with exposure to Fuel C. For the two fluorocarbons, the volume swelled between 12 and 15\%, while the fluorosilicone specimen expanded by $20 \%$. It is important to note that fluorosilicone is a mixture of both fluorocarbon and silicone rubber and the silicone fraction would be expected to produce in a higher volume swell than a straight fluorocarbon. The addition of $10 \%$ aggressive ethanol was observed to further increase the volume by another $5 \%$ for the fluorocarbons, but only caused a small $2 \%$ increase with the fluorosilicone. Increasing the ethanol content to $17 \%$ resulted in a slightly higher increase in volume for the fluorocarbons, but had no additional increase was observed for the fluorosilicone.

The addition of 16 and 24\% aggressive isobutanol produced additional volume change over the Fuel C baseline for the two fluorocarbons but at a level equal to or less than that achieved from the ethanol exposures. For the fluorosilicone specimen, isobutanol was observed to produce a slightly lower volume swell than Fuel C. Isobutanol concentration had little, if any, effect on the volume. 


\subsubsection{NBRs}

The volume and mass change results for the six nitrile rubbers are shown in Fig. 32. Although there is some variability according to rubber type, the relative volume swelling for test fuels containing ethanol and isobutanol was in agreement with the solubility analysis performed in Fig. 9. In general the specimens swelled between 17 and 25\% with exposure to Fuel C (depending on the type). Exposure to $10 \%$ aggressive ethanol increased the volume swell considerably, although raising the ethanol content to $15 \%$ produced only a small additional expansion. The volume expansion associated with oxygen equivalent levels of isobutanol was either the same or slightly less than that achieved with equivalent ethanol. Interestingly, the volume swell for all NBRs was observed to increase with increased ethanol content, whereas the volume swell was essentially unchanged between 16 and 24\% isobutanol. This observation was also noted for the fluoroelastomers.

Percent Mass Increase

Percent Volume Increase

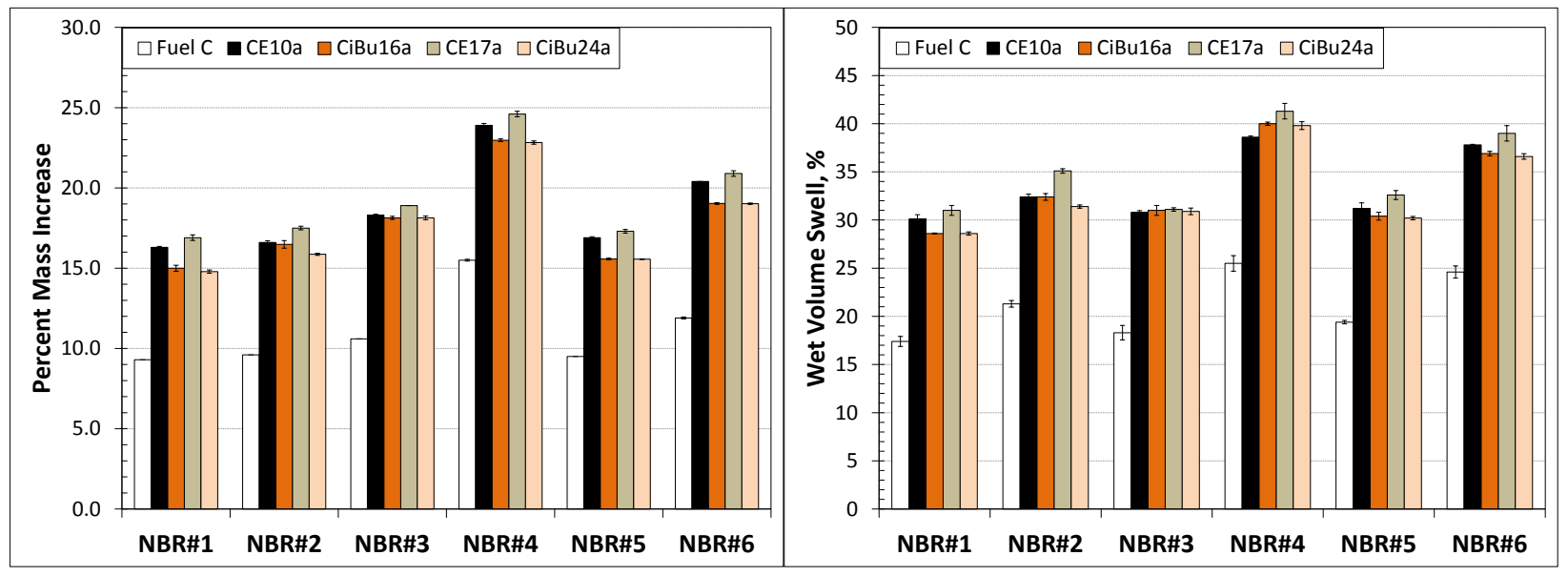

Fig. 32. Mass and volume increases for the NBRs exposed to the test fuel liquids for 4 weeks.

\subsubsection{Polyurethane, neoprene, SBR, and silicone rubbers}

A comparison of the ethanol and isobutanol swelling results for the remaining elastomers (polyurethane, neoprene, SBR, and silicone) is shown in Fig. 33. Each of these materials exhibited significantly higher swelling in the test fuels than either the fluoroelastomers or the NBR samples. This result was predicted by the solubility curves for neoprene, SBR, and silicone, but not for polyurethane.

Neoprene swelled approximately 65\% with exposure to Fuel C liquid, and adding $10 \%$ and $17 \%$ aggressive ethanol increased the volume by 79 and 75\%, respectively. The level of swell accompanying the isobutanol fuels was slightly lower than the results for equivalent levels of ethanol. These high levels of swelling roughly correspond to the moderate to high solubility predicted by solubility curves and interaction radius described in Fig. 11.

The volume of the SBR specimen expanded approximately $120 \%$ with Fuel C exposure. The addition of $10 \%$ aggressive ethanol increased the swelling to $130 \%$, but $17 \%$ ethanol lowered the value to around $125 \%$. The test fuels containing isobutanol produced lower swelling than the ethanol blends. In fact, CiBu16a produced the same level of swell as Fuel C, and CiBu24a produced 100\% swell, which was 20\% lower than the volume obtained with Fuel C. SBR was observed to be sensitive to the concentration of isobutanol; CiBu24a produced lower swelling than CiBu16a. It is important to note that high swelling was predicted by the solubility curves for SBR. 


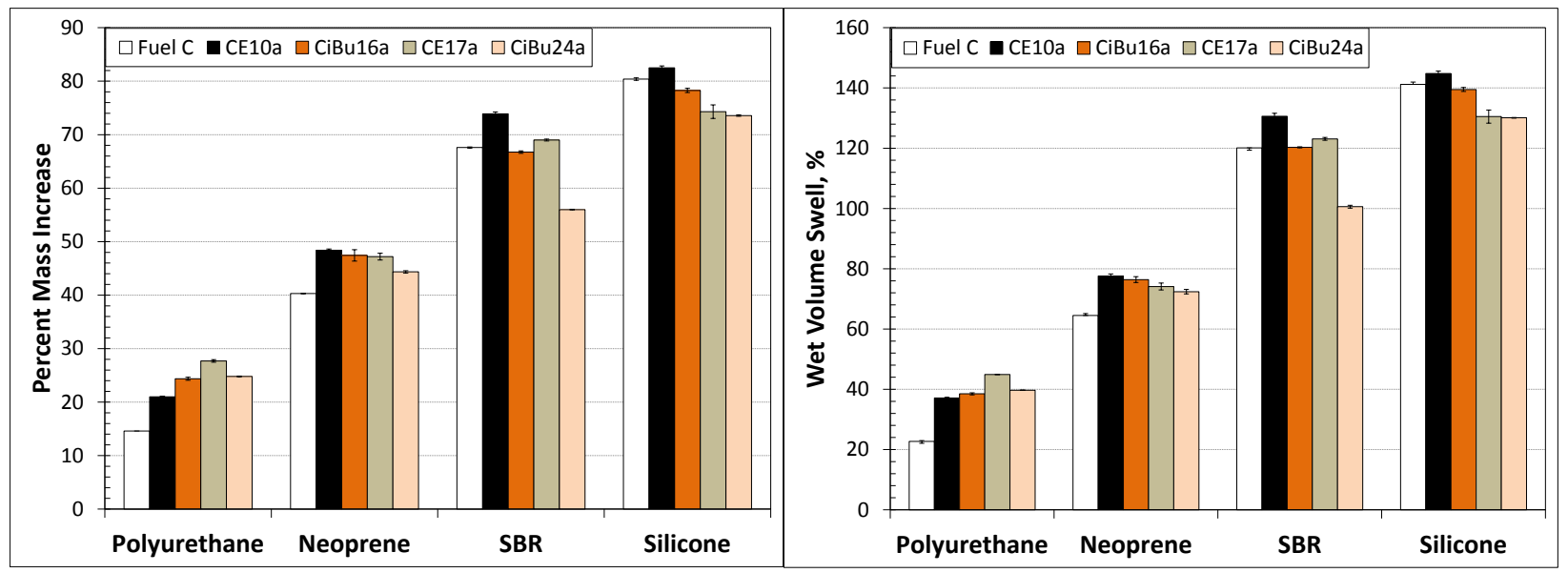

Fig. 33. Mass and volume increases for the polyurethane, neoprene, SBR, and silicone specimens exposed to the test fuel liquids for 4 weeks.

Silicone rubber exhibited the highest level of swelling of the elastomers studied, which is not surprising since the difference between the interaction radius and the $d_{S}$ values was highest for this material (as shown in Fig. 13). Fuel C caused the volume to expand by 140\%. The addition of $10 \%$ aggressive ethanol slightly raised this value, but $17 \%$ aggressive ethanol decreased the swell to $130 \%$ (10 percentage points less than Fuel C). The addition of 16\% aggressive isobutanol produced the same level of swelling as Fuel C, while $24 \%$ aggressive isobutanol dropped the total swell to $130 \%$, which is the same extent of swelling as observed with CE17a.

Based on the solubility curves for polyurethane (Fig. 12), very low levels of swelling in the test fuels would be expected. In actuality, polyurethane was noted to swell over $20 \%$ from the baseline condition with exposure to Fuel C; with the addition of 10 and $17 \%$ aggressive ethanol, this value was raised to 37 and $45 \%$, respectively. These volume expansions are considered moderate and do not correlate with the solubility curves. When isobutanol was added to Fuel C (either as CiBu16a and CiBu24a), the volume of the polyurethane specimens was increased $40 \%$. Previous studies have indicated that polyurethane will chemically react and degrade with ethanol. Since the solubility curves do not account for chemical reactivity, the chemical degradation associated with polyurethane likely contributed to the higher than predicted level of swelling.

\subsubsection{Wet Hardness (or Softening)}

Upon removal from the test fuels, the elastomer specimens were measured for hardness using the Shore A method. The specimens were maintained in the wetted state just before placement on the apparatus for hardness measurement. The hardness measurement (in the wetted condition) is expected to be lower than the original baseline value since the adsorbed test fuel liquids would not offer any resistance to penetration. (Reduction in hardness can also be expressed as an increase in softening.) In general swelling is almost always accompanied by an increase in softening, and this hardness decrease is roughly proportional to the volume expansion. This effect can be seen in Fig. 34, whereby all of the elastomer samples show a reduced hardness (from the baseline condition) with exposure to the test fuels. Except for polyurethane, most the softening can be attributed to the Fuel C, though a marginal increase in softening did occur with the addition of ethanol or isobutanol. 


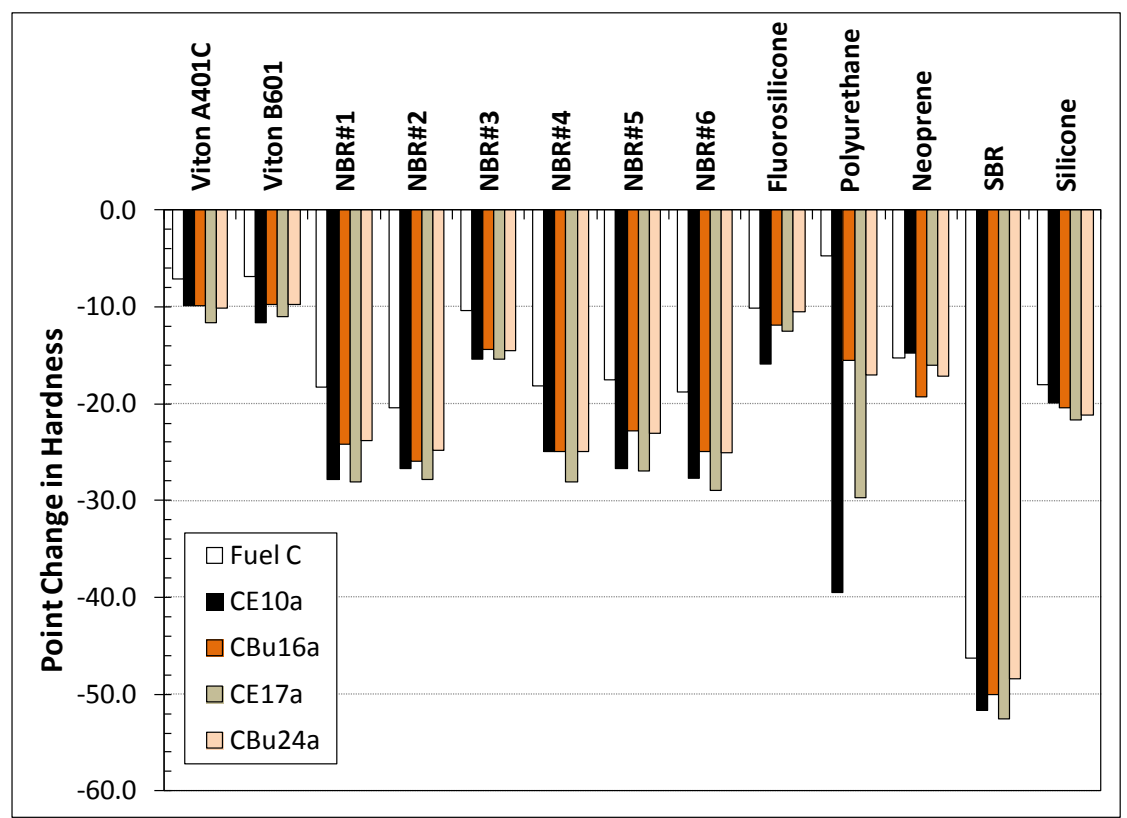

Fig. 34. Hardness decrease (softening) of elastomers exposed to the liquid test fuels. Specimens were tested while wet.

\subsubsection{Fluoroelastomers}

The wet hardness results for the three fluoroelastomers are shown in Fig. 35. The two fluorocarbons exhibited similar declines in hardness (from the baseline condition).

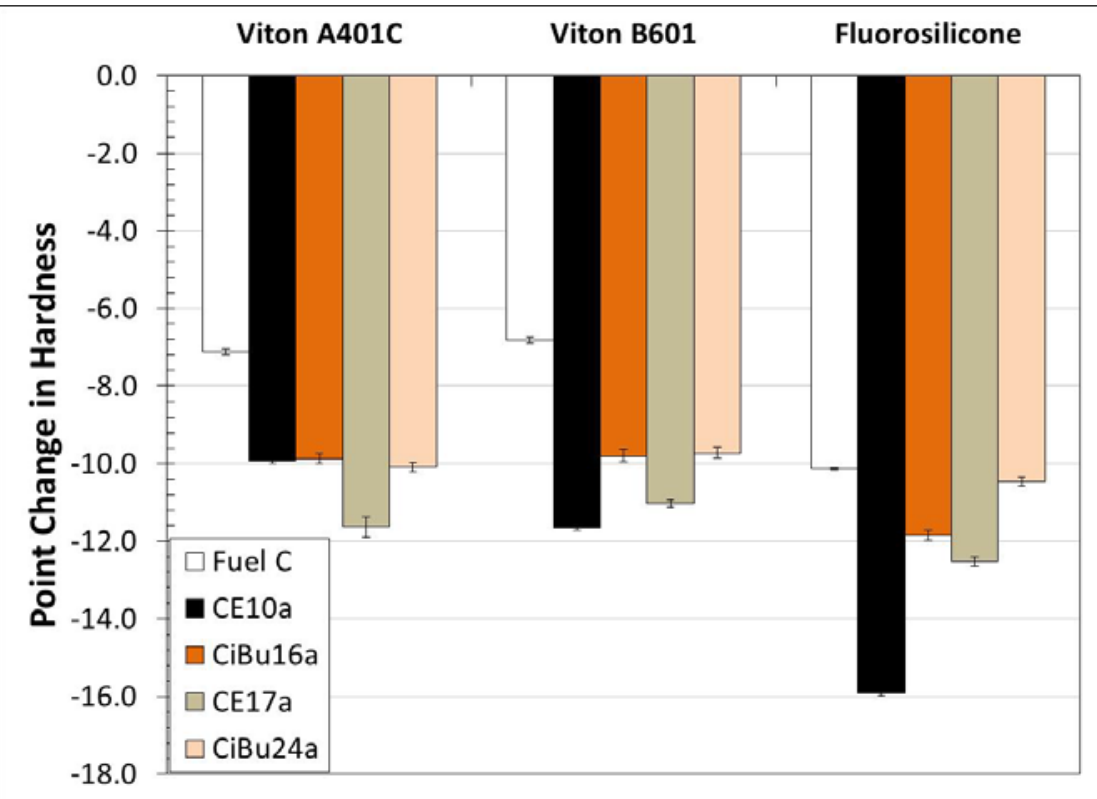

Fig. 35. Hardness decrease (softening) of the fluoroelastomer specimens exposed to the liquid test fuels. Specimens were tested while wet.

The added ethanol and isobutanol resulted in an additional 3 to 5 point decrease in hardness (which is quite low relative to the absolute baseline hardness value). In general the aggressive isobutanol additions did not produce as much softening as did the fuel formulations containing aggressive ethanol. The 
fluorosilicone specimen exhibited more decline in hardness than the fluorocarbons, but these values (especially when compared to Fuel C) are not considered noteworthy.

\subsubsection{NBRs}

After the fluoroelastomers, the NBR specimens (as a group) showed the lowest drop in wet hardness from the baseline condition. The behaviors for the different NBR specimens were similar to each other as shown in Fig. 36, although the marine grade (NBR\#3) exhibited significantly less softening than the other types. The NBRs (excluding NBR\#3) declined 15 to 20 points with exposure to Fuel C in the wetted state, while NBR\#3 declined by 10 points. This drop in hardness is significant relative to the initial hardness. For each NBR type, the addition of alcohol (ethanol or isobutanol) further decreased the hardness by 5 to 10 points, and in most cases the aggressive ethanol was a more effective softening agent than aggressive isobutanol.

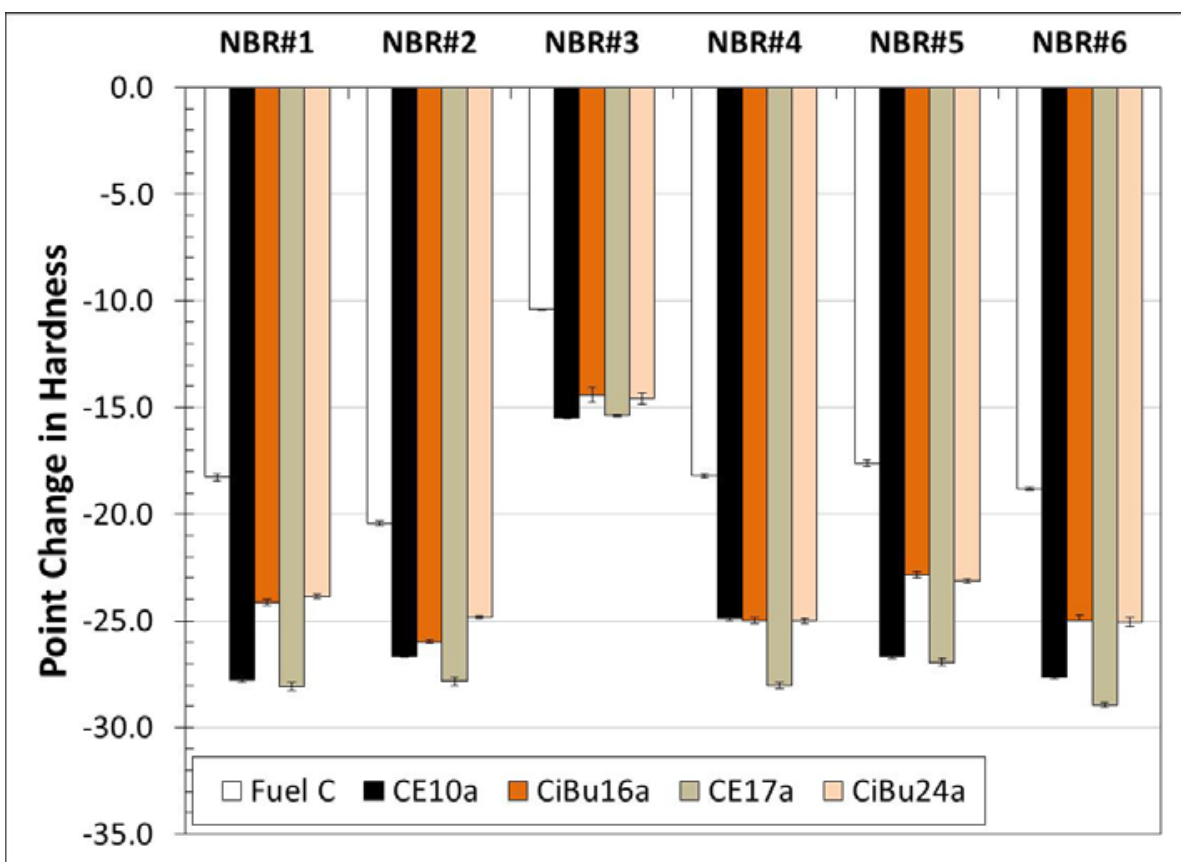

Fig. 36. Hardness decrease (softening) of the six NBR specimens exposed to the liquid test fuels.

\subsubsection{Polyurethane, neoprene, SBR, and silicone rubbers}

The hardness results for polyurethane, neoprene, SBR, and silicone rubbers (while wet) are shown in Fig. 37. The chemical structures for each of these materials are quite different, and this difference is reflected in the extent of softening experienced for each test fuel composition. Exposure to Fuel C produced the least extent of softening in each of the elastomer materials. 


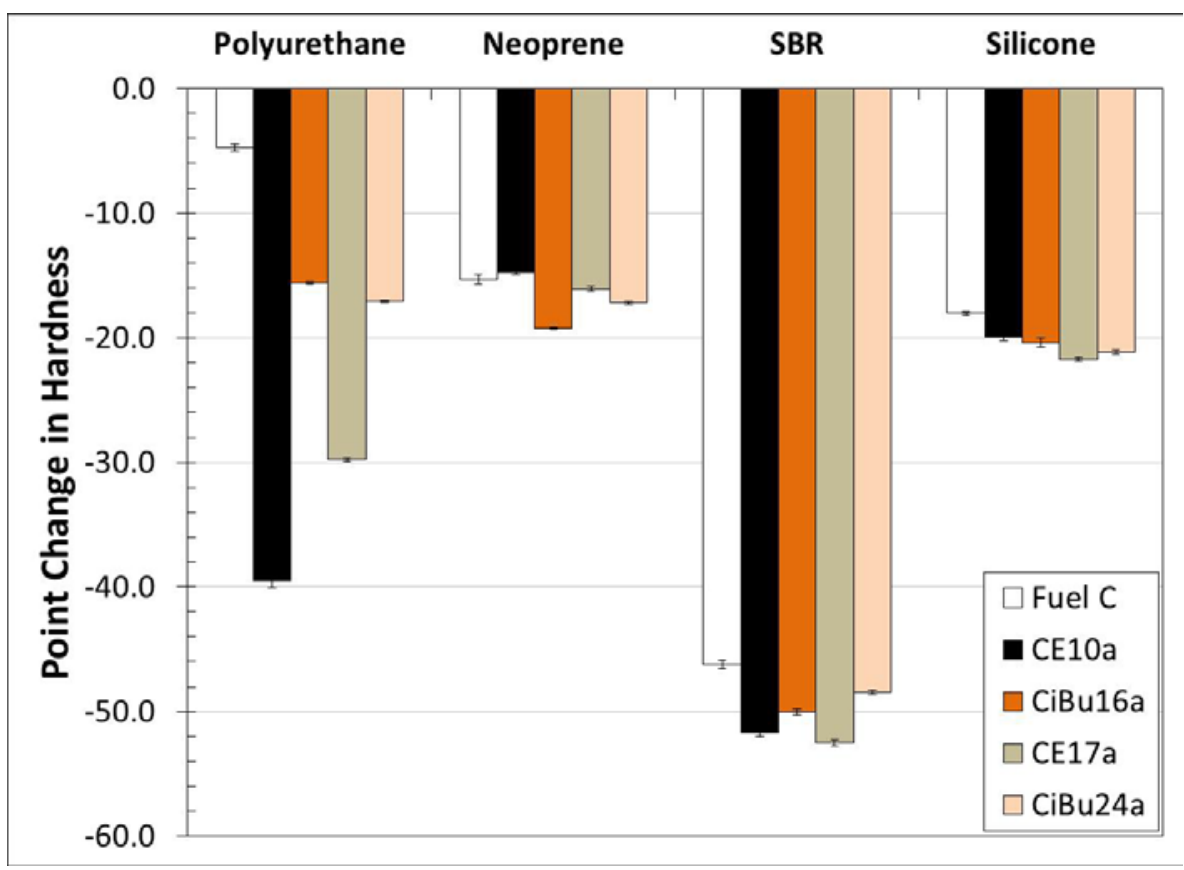

Fig. 37. Hardness decrease (softening) of polyurethane, neoprene, SBR, and silicone rubber specimens exposed to the liquid test fuels.

Polyurethane was unique among all of the elastomers evaluated in this study in that its hardness was most affected by the alcohols. However, the additions of 10 and 15\% aggressive ethanol caused the hardness to drop 40 and 30 points, respectively. Aggressive isobutanol dropped the hardness between 15 and 17 points, which is a significantly lower decrease than the results for the ethanol blends. The implication is that polyurethane may be more compatible to gasoline containing isobutanol than ethanol.

The hardness values for neoprene dropped around 15 points with exposure to the test fuels. Most of this decrease is attributed to Fuel $\mathrm{C}$ as the addition of either alcohol did not produce any significant additional change. The SBR specimen experienced the highest drop in hardness with exposure to the test fuels. As with the other elastomer types, excluding polyurethane, Fuel $\mathrm{C}$ appears to be the component most responsible for the decline in hardness. The two alcohol additives did produce a small additional decrease in hardness, with aggressive ethanol causing slightly more softening than aggressive isobutanol. The original hardness for silicone rubber was reduced 20 points with exposure to Fuel C. The alcohols provided a small (almost insignificant) additional contribution.

\subsubsection{Dried mass and volume for wetted specimens}

The wetted specimens were dried for 20 hours at $60^{\circ} \mathrm{C}$ to understand the material response to conditions in which the fuel is no longer in contact, such as an emptied storage tank and piping. The volume (and mass) change following drying is an important property since it can indicate the extent of dissolution and possible extraction of important elastomer components, such as plasticizers, ultraviolet protectors, ozone protectors, and antioxidants. The volume change was compared to the starting original condition, and these results are shown in Figs. 38-40 for the different elastomers. Interestingly, the mass and volume were higher than the baseline condition for the two fluorocarbons, while the other elastomers were either unaffected or experienced a loss of mass/volume. Silicone rubber did experience a small volume contraction after being dried. 

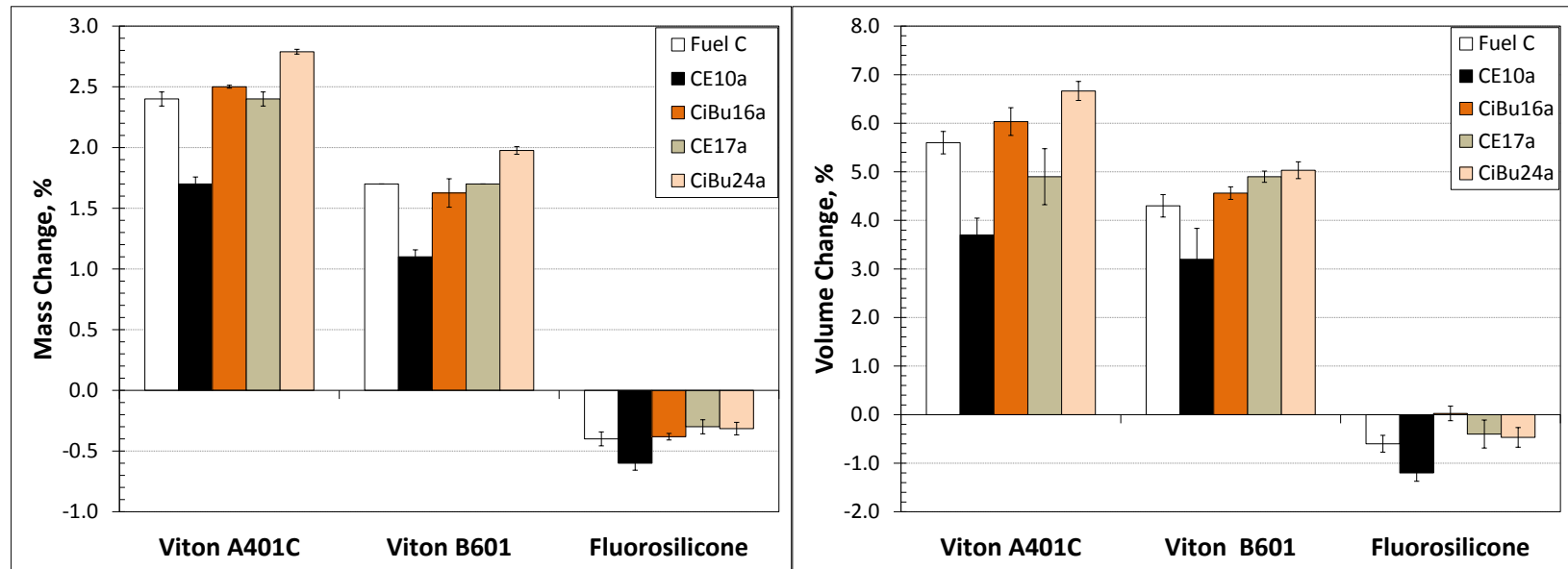

Fig. 38. Volume change of fluoroelastomers exposed to the liquid test fuels after drying at $60^{\circ} \mathrm{C}$ for 20 hours.

Percent Mass Change

Percent Volume Change
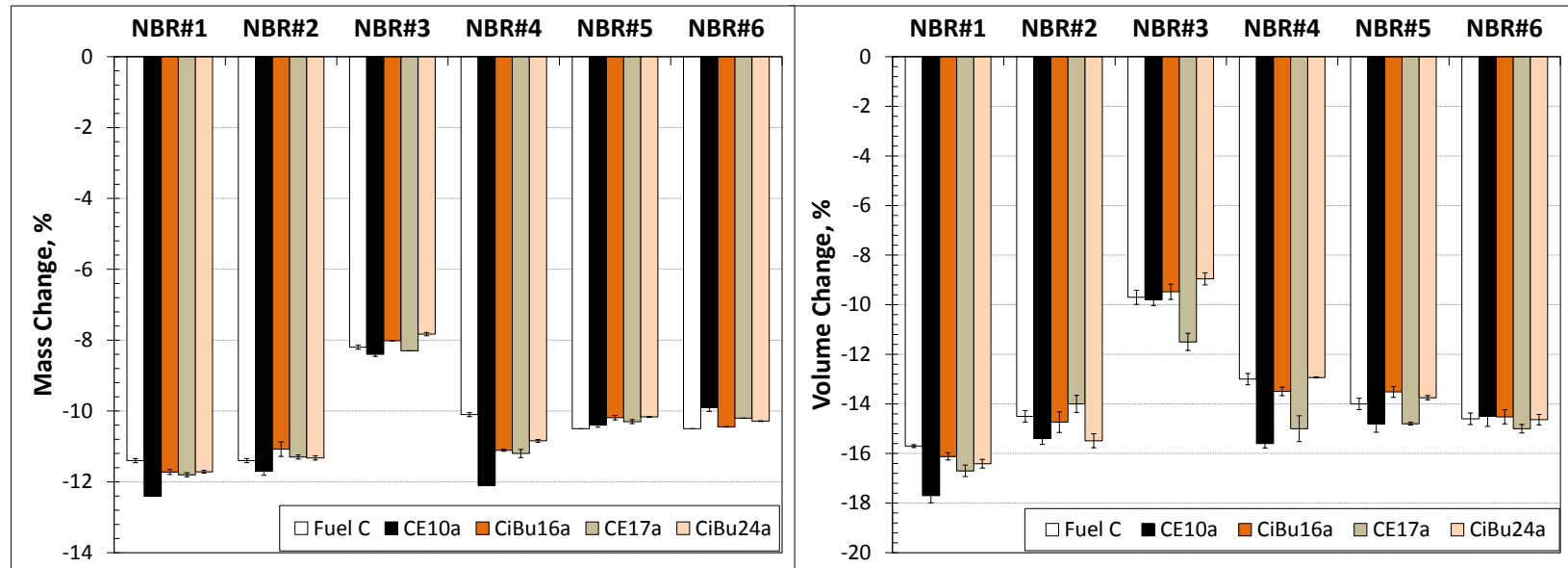

Fig. 39. Volume change of NBRs exposed to the liquid test fuels after drying at $60^{\circ} \mathrm{C}$ for 20 hours.

Percent Mass Change

Percent Volume Change

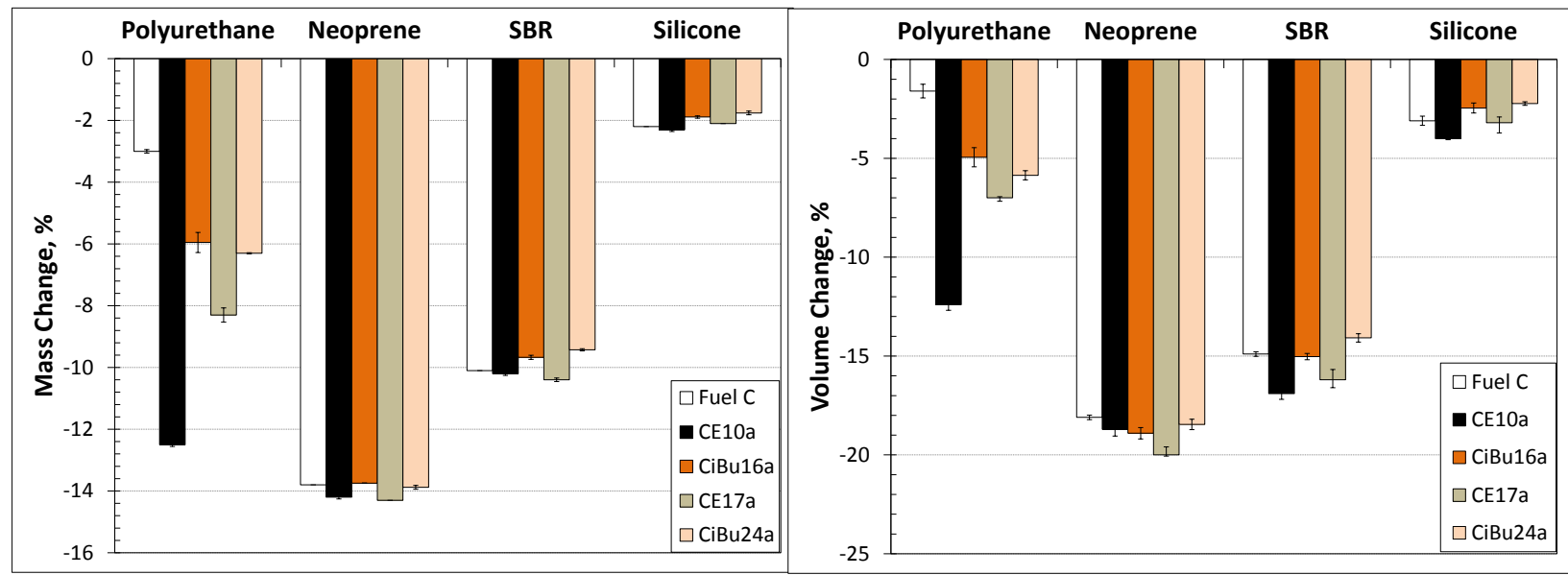

Fig. 40. Volume change of polyurethane, neoprene, SBR, and silicone rubber specimens exposed to the liquid test fuels after drying at $60^{\circ} \mathrm{C}$ for 20 hours. 


\subsubsection{Fluoroelastomers}

The volume and mass change for the two fluorocarbons and fluorosilicone specimen following dry-out is shown in Fig. 38. For the two fluorocarbons, the volume and mass swell were in similar proportion to the wetted specimens (see Fig. 31), which indicates that the mass/volume increase is due to retained test fuel liquids. The two fluorocarbons exhibited a small volume increase with each test fuel following dry-out. This effect was also noted by other researchers. Fuel C was a primary contributor to this retained volume and, interestingly, the volume swell was lower for CE10a and CE17a, respectively. The resulting retained volume expansion varied slightly with ethanol concentration for the two fluorocarbons. Viton A401C exhibited a slightly lower dried volume for the ethanol fuel blends. For Viton B601, the dry-out volume produced with CE10a was slightly less than that achieved for Fuel C, while that with CE17a was slightly higher. The addition of isobutanol slightly increased the dry-out volume, and this effect is attributed to the larger molecular size of isobutanol, whose lower vapor pressure (relative to ethanol) requires more energy to be extracted from the host rubber.

\subsubsection{NBRs}

All of the NBRs underwent significant volume contraction (between 10 and 18\%) following dry-out as shown in Fig. 39. All of the specimens, except for the marine grade (NBR\#3) shrank between 13 and $18 \%$. The results suggest that Fuel $\mathrm{C}$ is the component most responsible for this contraction, although ethanol had a minor additional contribution for NBR\#1 and NBR\#4. The specimens exposed to the test fuels containing isobutanol exhibited a similar volume contraction as those exposed to Fuel C. This result suggests that isobutanol has an insignificant effect on the overall shrinkage for these materials. The removal of a significant amount of NBR mass and volume indicates that dissolution and extraction of one or more components had occurred. The volume reduction for the marine-grade NBR was around $10 \%$, which was measurably lower than that of the other NBRs. The other NBR grades were found to exhibit similar performance in volume reduction for each test fuel.

\subsubsection{Polyurethane, neoprene, SBR, and silicone rubbers}

Consistent with the wet hardness results, polyurethane was the elastomer most affected by alcohol. As shown in Fig. 40, polyurethane underwent a 1-2\% decrease in volume following exposure to Fuel C, but exposure to CE10a and CE17a resulted in dry-out shrinkages of 12 and 7\%, respectively. In contrast, the added isobutanol caused polyurethane to shrink around 5\%. The reduced volume is an indication that isobutanol is more compatible with polyurethane than ethanol. The fact that the mass and volume losses were roughly equivalent is an indication that the polyurethane itself, not an additive, was dissolved and extracted.

Neoprene exhibited the highest level of shrinkage (following dry-out) of the elastomers tested. It was observed to shrink around 18 to 20\% following dry-out after being exposed to the test fuel liquids. In this case, Fuel $\mathrm{C}$ appears to be the test fuel component most responsible for this effect, with ethanol producing a small additional contribution. The test fuels containing isobutanol produced similar results as Fuel C.

SBR underwent considerable shrinkage (15\%) when dried after Fuel C immersion. Aggressive ethanol produced an extra $2 \%$ volume contract, while isobutanol did not provide any added effect. The results suggest that neoprene is slightly more compatible with gasoline containing isobutanol than with ethanol.

Silicone rubber (like the fluorosilicone specimen) underwent minimal shrinkage following dry-out. However, the test fuels containing ethanol did produce a small but significant contribution to the shrinkage, while the test fuels containing isobutanol provided the best results. 


\subsubsection{Hardness Change Following Drying for Wetted and Vapor-Phase Specimens}

The change in hardness following drying is another key property used to assess whether structural or compositional changes have taken place in the elastomer from the exposure to the test fuels. If the elastomer was not compounded with additional components, then a volume change would be expected to be accompanied by a change in hardness. However, extraction of a plasticizer (such as phthalates) would be expected to cause shrinkage (following dry-out) accompanied by an increase the hardness. Shrinkage accompanied by a loss in hardness is indicative of chemical degradation of the polymer.

The results in Figs. 41-43 reveal that several elastomers, such as the NBRs and neoprene, experienced significant embrittlement after drying, yet the fluoroelastomers exhibited a small drop in hardness. The sensitivity to fuel chemistry was also noted for polyurethane, SBR, and some NBR specimens.

Liquid Exposures

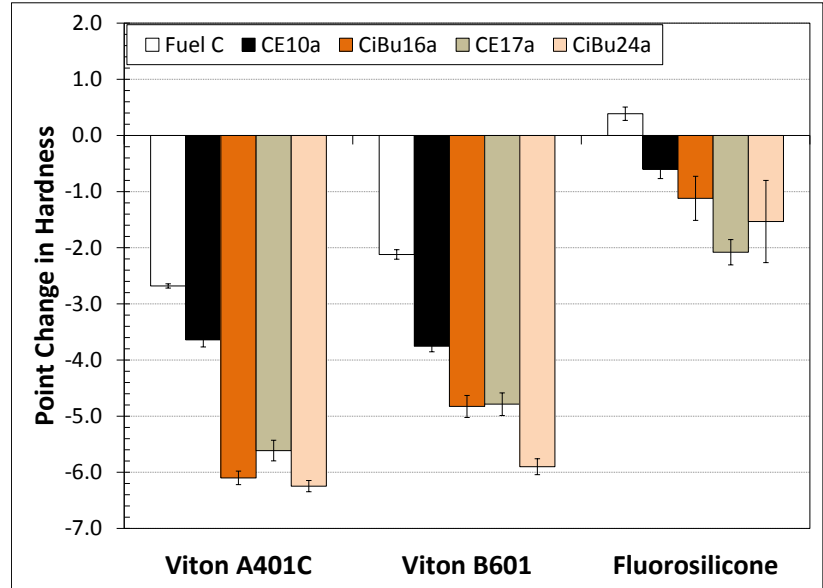

Vapor Exposures

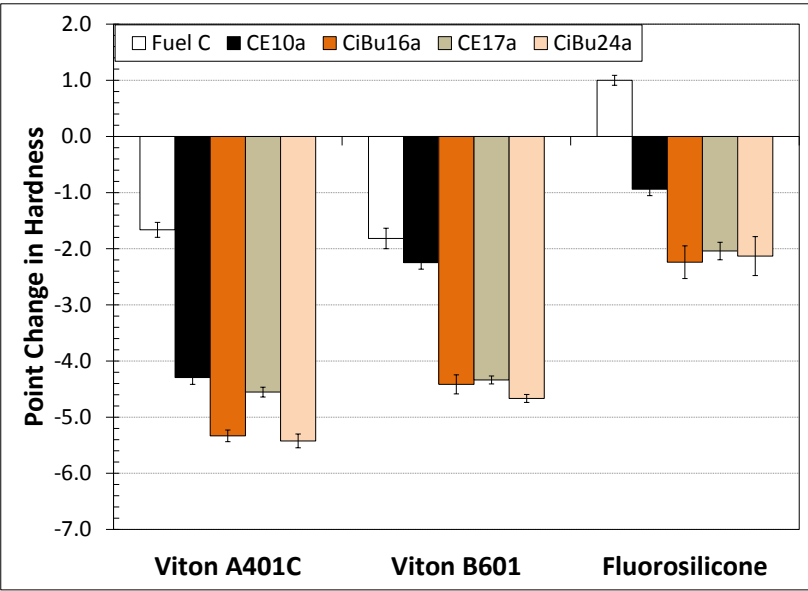

Fig. 41. Point change in hardness (after drying) from the baseline condition for fluoroelastomers in the liquid and vapor phases of the test fuel liquids.

Liquid Exposures

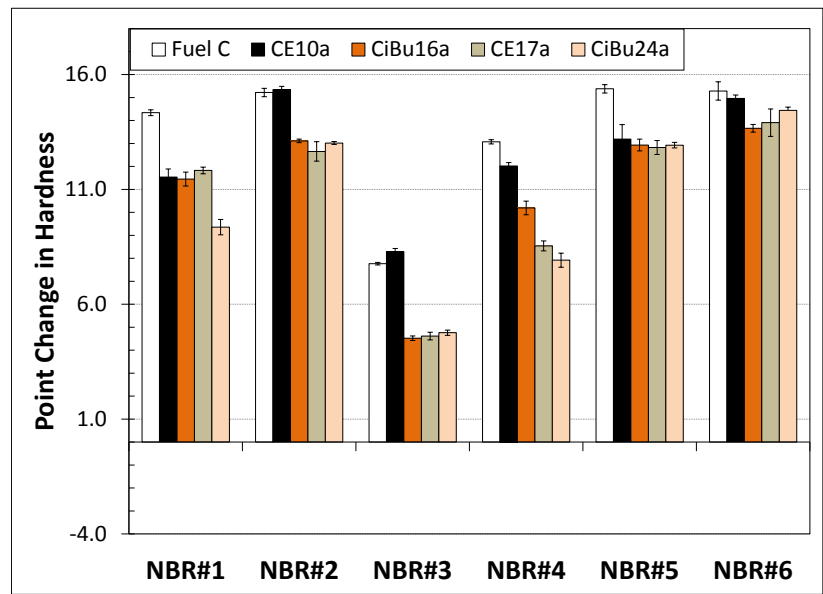

Vapor Exposures

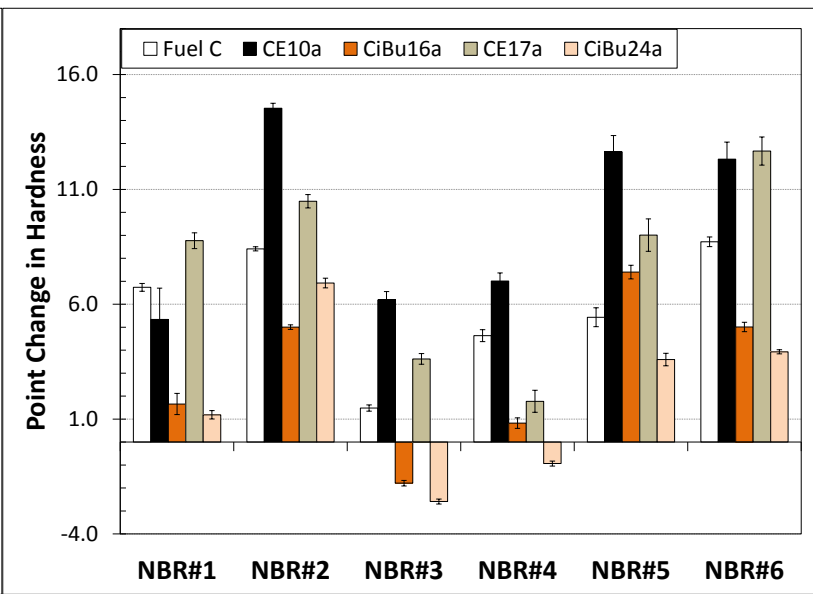

Fig. 42. Point change in hardness (after drying) from the baseline condition for NBRs in the liquid and vapor phases of the test fuel liquids. 


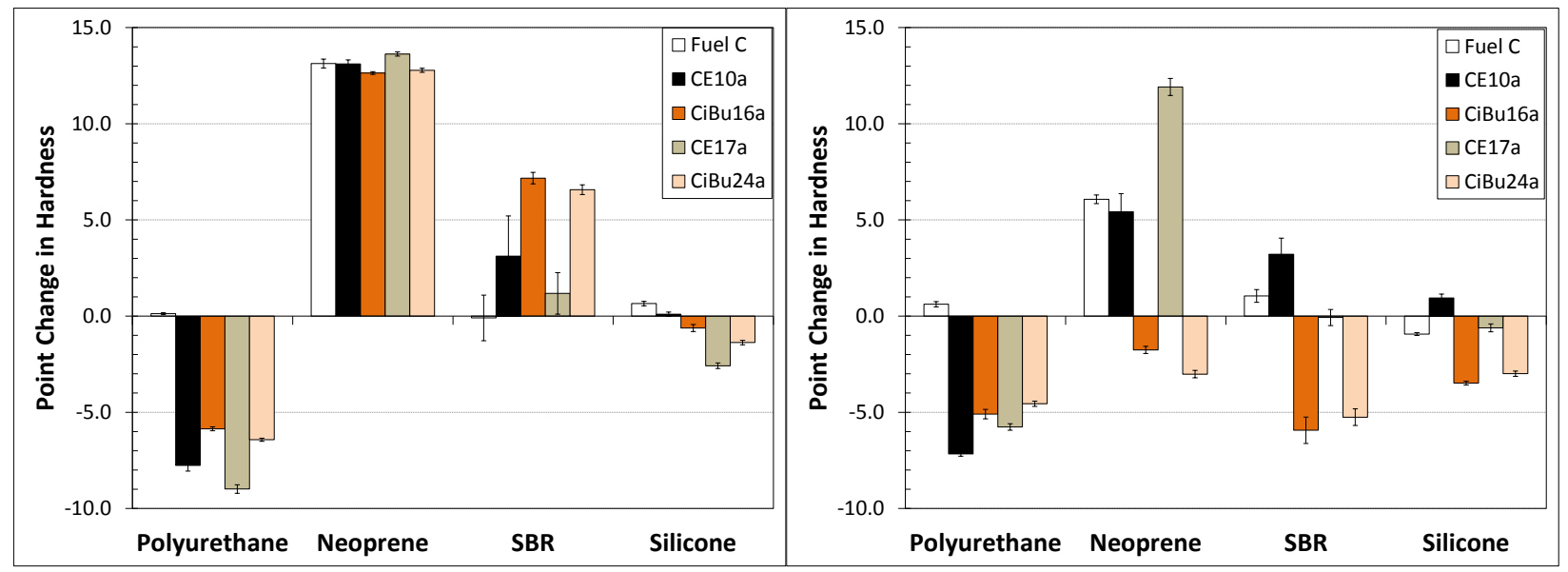

Fig. 43. Point change in hardness (after drying) from the baseline condition for polyurethane, neoprene, SBR, and silicone in the liquid and vapor phases of the test fuels.

The change in hardness values for those fluoroelastomer specimens exposed to the test fuel vapors was quite similar (albeit to a slightly smaller extent) to the change in hardness observed for those previously wetted in the same test fuels. The implication is that the test fuel components were able to effectively permeate into the fluorocarbon structure to affect the hardness and the drying process was not able to effectively remove the test fuel components. This effect is remarkable given that the vapor pressure of ethanol is higher than that of isobutanol, so the concentration of ethanol in the headspace region during the ethanol exposures would be expected to be higher than the isobutanol concentration in the counterpart experiments.

\subsubsection{Fluoroelastomers}

The dry-out hardness results for the three fluoroelastomers exhibited small losses in hardness as shown in Fig. 41. The two fluorocarbons show a slight 2 to 3 point decrease in hardness (softening) upon exposure to Fuel C. The dry-out hardness for the fluorocarbons declined an additional 3 points for the test fuels containing aggressive ethanol and aggressive isobutanol. The test fuels containing isobutanol produced a slightly higher softening (hardness decline) than does ethanol, and this added effect is attributed to the increased retention of isobutanol in the dried specimens as indicated previously in Fig. 38. The fluorosilicone specimen was relatively unaffected by the test fuels; a 2 point drop in hardness was noted for the fuels containing ethanol and isobutanol, but this number is probably too low to be considered significant.

\subsubsection{NBRs}

The dry-out hardness changes (from the baseline condition) for the NBR specimens are shown in Fig. 42. Significant embrittlement (hardness increase) was noted for each specimen; however, the marine grade was much less affected than the other types. Generally the highest increase in hardness was observed for specimens exposed to Fuel C. The addition of $10 \%$ aggressive ethanol had little additional effect on the dry-out hardness for NBR\#2, NBR\#3, NBR\#4, and NBR\#6, but for NBR\#1 and NBR\#5, the extent of embrittlement was slightly lower with exposure to CE10a. The addition of 16 and 24\% aggressive isobutanol and $17 \%$ aggressive ethanol lowered the extent of embrittlement to a small degree, although for NBR\#3 the drop in hardness was around half that achieved for Fuel C and CE10a. In general 16\% isobutanol produced a lower level of embrittlement than CE10a, but there was no significant differences in hardness change between $24 \%$ isobutanol and CE17a. 


\subsubsection{Polyurethane, neoprene, SBR, and silicone}

The dry-out hardness values for polyurethane, neoprene, SBR, and silicone are shown in Fig. 43. Polyurethane dry-out hardness was unaffected by Fuel C, but this material did post a small drop with exposure with added ethanol and, to a lesser extent isobutanol. Softening in the dried state is an indication of a possible degradation when viewed along with the accompanying shrinkage and wet hardness results.

Figure 43 also shows that neoprene experienced significant embrittlement when exposed to Fuel C. However, the addition of aggressive ethanol or aggressive isobutanol had no observable added effect. SBR did not appear to be affected by Fuel C; however, the addition of ethanol caused a slight increase in hardness, and isobutanol raised the hardness by 5 points (which is still considered low). The dry-out hardness of silicone was essentially unaffected by the test fuels, and it (along with fluorosilicone) were the two elastomers least affected.

\subsubsection{Dynamic Mechanical Analysis Results for Elastomers}

Dynamic mechanical analysis measures the elastic storage modulus in polymers over a wide temperature change. It is a useful means of detecting structural changes, particularly those that affect a change in molecular relaxation. Molecular relaxation governs many physical properties of a polymer, especially the point at which a polymer transitions from a stiff glassy state to a pliable rubbery state; this parameter is the glass transition temperature $\left(T_{g}\right)$. The full DMA curves for each elastomer specimen are shown in Appendix A.

For the elastomer materials, the glass transition temperatures were affected by the test fuels, as shown in Figs. 44-46. However, the direction of the shift in $\mathrm{T}_{\mathrm{g}}$ varied according to material type. For instance, the two fluorocarbons (Viton A401C and Viton B601C) both exhibited a decrease in glass transition temperature with exposure to the test fuels. The primary cause for this downward shift was Fuel C, as the additions of aggressive ethanol or aggressive isobutanol do not appear to have any additional impact on $\mathrm{T}_{\mathrm{g}}$ beyond that observed for Fuel C. This reduction in the glass transition temperature is associated with the retention of the test fuel liquid in the fluoroelastomer structure and the resulting expansion in volume. This volume increase allows molecular relaxation to occur at lower temperatures than unexposed elastomers. For fluorosilicone, the glass transition temperature appeared to be unaffected when exposed to the test fuels.

Liquid Exposures

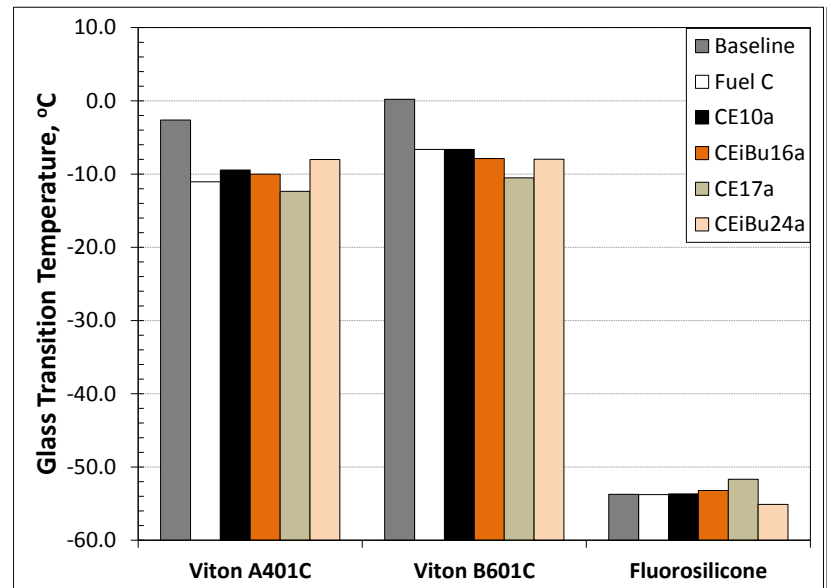

Vapor Exposures

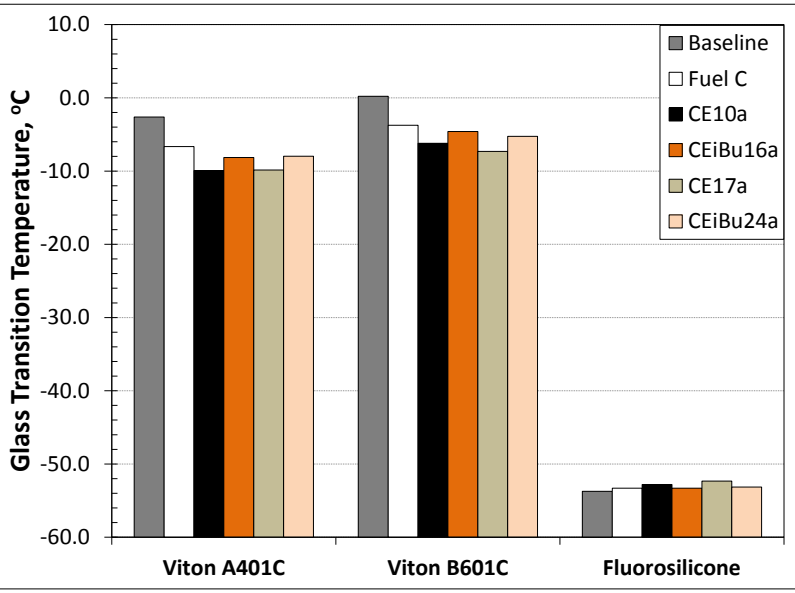

Fig. 44. Glass transition temperature results for fluoroelastomers exposed to the test fuel liquid and vapor regions. 


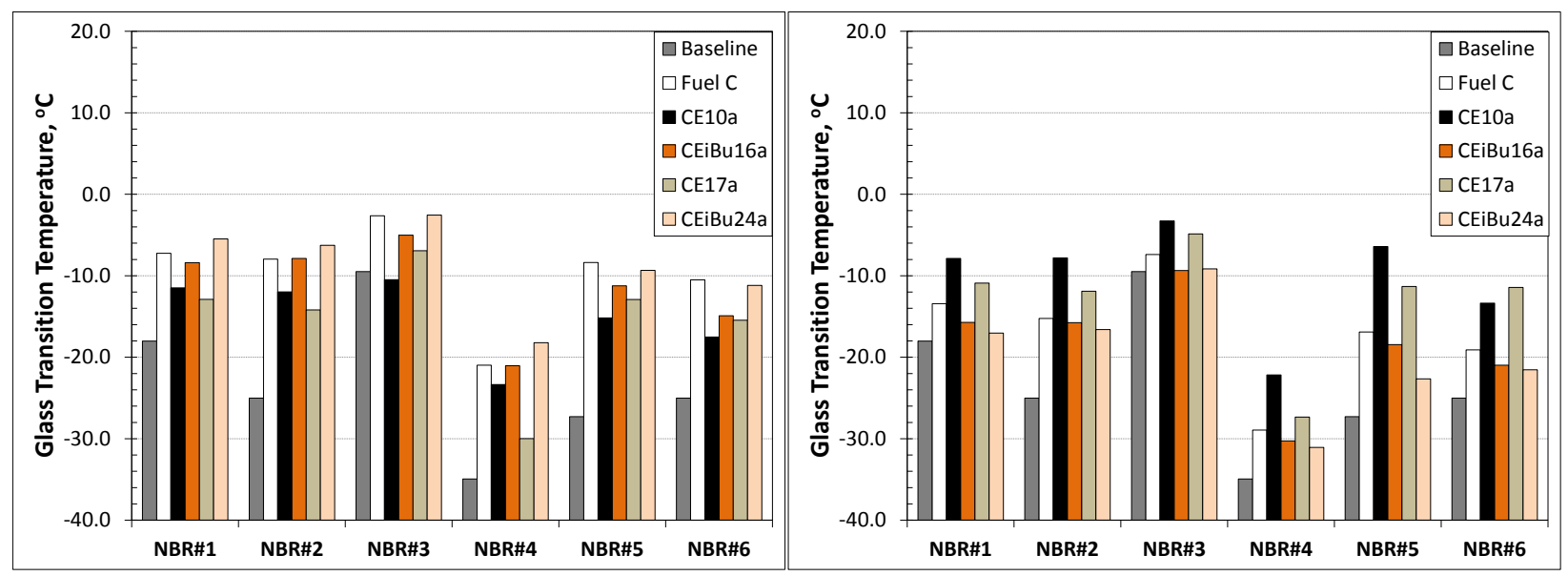

Fig. 45. Glass transition temperature results for NBRs exposed the test fuel liquid and vapor regions.

Liquid Exposures

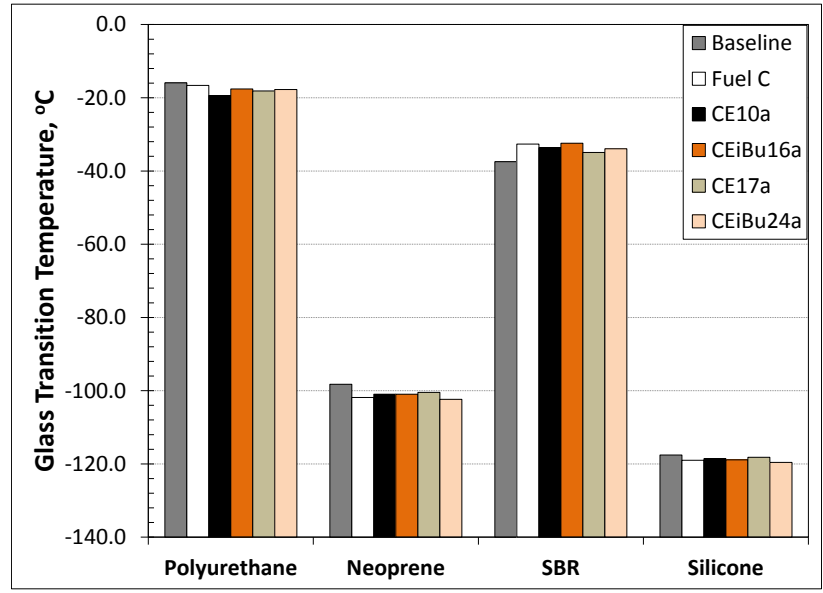

Vapor Exposures

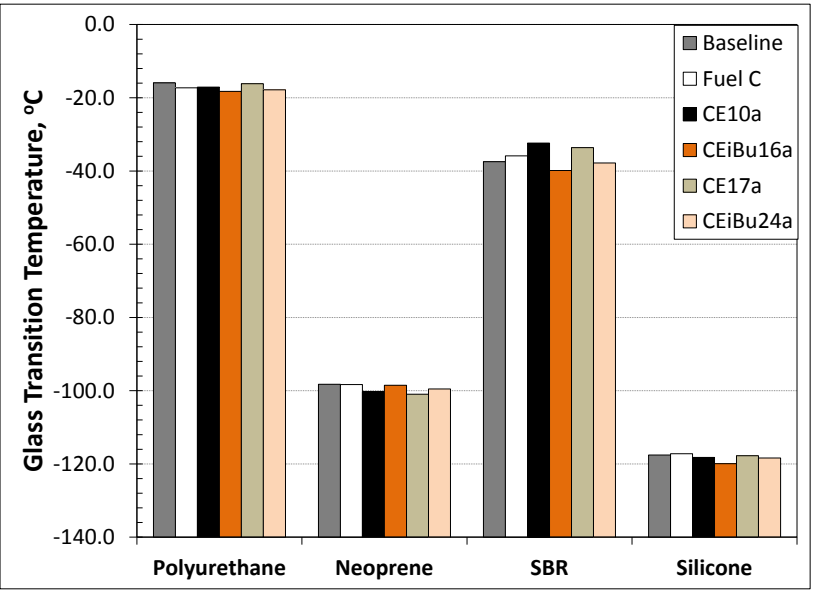

Fig. 46. Glass transition temperature results for polyurethane, neoprene, SBR, and silicone rubbers exposed the test fuel liquid and vapor regions.

In contrast to the fluorocarbons, the $\mathrm{T}_{\mathrm{g}}$ 's for the six NBR grades were raised to higher temperatures as shown in Fig. 45. This increase was significant for all except NBR\#3 (the marine-grade formulation), following exposure to the test fuels. Each NBR specimen exhibited reduced volume and increased hardness following drying, which is an indication that the plasticizer had been extracted by the test fuels. For the specimens exposed directly to the test fuel liquids, Fuel C raised the glass transition temperature by the highest amount, compared to the original baseline condition. The test fuels containing ethanol or isobutanol also raised the transition temperature, but to a value slightly less than that for Fuel C. For those specimens exposed to the vapor-phase regions, the $\mathrm{T}_{\mathrm{g}}$ was also increased, albeit to a lesser extent than those exposed to the test fuel liquids. An interesting feature associated with the vapor exposures is that CE10a and CE17a caused the highest shift in $\mathrm{T}_{\mathrm{g}}$, whereas Fuel $\mathrm{C}$ caused the greatest shift for liquid exposures. Vapor exposure from CiBu16aand CiBu24a test fuels produced the lowest change in $\mathrm{T}_{\mathrm{g}}$ from the original baseline condition. In fact, for several NBRs (NBR\#2, NBR\#4, NBR\#5, and NBR\#6), there was essentially no change in the glass transition temperatures with exposure to CiBu24a from the original unexposed condition. For NBR\#1 and NBR\#3, the glass transition temperature was actually lowered by a small (but noticeable) amount with exposure to CiBu24a vapor phase. It is important to keep in mind that 
the ratio of the Fuel C components and the alcohols of the vapor phase will not match the relative fractions of the liquid phase. Whereas ethanol has a vapor pressure slightly higher than isooctane, isobutanol has a much lower vapor pressure than Fuel C. Therefore we would expect a higher fraction of ethanol in the vapor phase followed by isooctane and toluene. In contrast, for the isobutanol-fuel blends, the concentration of isobutanol would be expected to be reduced in the vapor region relative to the Fuel $\mathrm{C}$ components (isooctane and toluene).

The glass transition temperatures for polyurethane, neoprene, SBR, and silicone rubbers are shown in Fig. 46. For these rubber types, the representative specimens showed no significant change to $T_{g}$ when exposed to the test fuels, either in liquid or vapor form.

\subsection{PLASTICS AND CORK}

The plastic and cork specimens were removed from the test fuels after 16 weeks of exposure. Longer exposure time is necessary to achieve complete fuel saturation for plastics. The wetted specimens were quickly transferred to a container partially filled with test fluid to prevent drying, while the headspace samples were placed inside a plastic bag. The handling and testing protocol for the plastic specimens followed that used for the elastomer specimens. Unlike the elastomer materials, the plastic specimens were not exposed to CE10a and CE17a test fuels, so there is no oxygen equivalent comparison to CiBu16a and CiBu24a. The only ethanol test fuel available for comparison is CE25a, although previous analysis of the elastomers exposed to ethanol blended test fuels showed that CE25a produced similar levels of property changes to CE10a and CE17a.

The results for the plastic materials are grouped together according to their intended application to maintain the convention used in earlier sections of this report. However, none of the epoxy resins survived intact following exposure of the test fuel liquids or vapors. This result was not expected, since the solubility analysis indicated that epoxies would exhibit low to moderate solubility with the test fuel formulations. It is important to add that there are multitudes of epoxy types and curing processes, so it is likely that the HSPs selected for the solubility analysis were not accurate for the types included in this investigation.

In addition to the plastics, two rubber-impregnated cork materials were included alongside the plastic materials. Rubber is typically added to cork to improve the mechanical properties and overall durability. Cork impregnated with NBR (cork/NBR) was exposed to Fuel C, CE25a, CiBu16a, and CiBu24a. After completing the CE25a and Fuel C studies, it was discovered that cork impregnated with epichlorohydrin (cork/ECO) was used as a gasket material in some fuel dispenser hardware. While it was not possible to repeat the CE25a and Fuel $\mathrm{C}$ studies with this material, it was added to the isobutanol test fuels (CiBu16a and $\mathrm{CiBu} 24 \mathrm{a}$ ) to characterize performance with these fuels.

\subsubsection{Wet Mass and Volume}

It is important to note that plastics typically have higher molecular densities than elastomers. For many elastomers porosity is further added to the material to produce a foam structure, which, in turn, reduces weight and cost, and allows compression-based sealing to occur at low loads. The higher densities and lack of porosity cause these materials to swell less than elastomers when exposed to the test fuels. In addition, the interaction radii for all of the plastic materials (except the resins) were much lower than the radii for the elastomers, which indicates that these materials, thermodynamically speaking, are less soluble for a given solvent than most elastomers. Another key difference is that plastics are not normally compounded with additives to improve flexibility, so plasticizer extraction will not be a concern for most of these materials. This study does include two plastic materials that are co-polymers: one is PETG, which contains PET and an additional polymer component, the other material is a POM copolymer. 


\subsubsection{Permeation barrier plastics}

The changes in wet volume and mass for the plastic materials are shown in Figs. 47-50. The density range for these materials was 1.4 to $2.2 \mathrm{~g} / \mathrm{cc}$, which was higher than that of the other plastic materials evaluated in this study. The volume and mass changes for permeation barrier materials exhibited negligible to very low swelling and mass gain as shown in Fig. 47. As predicted by their respective solubility curves, the PPS, PET, and PVDF specimens did not undergo significant mass or volume swell with exposure to the test fuel liquids. In contrast, the solubility curve for PTFE (Fig. 15) indicated that moderate swelling would have occurred for this material in the test fuels, but that was not the case as PTFE showed negligible swelling. The implication is that the HSPs for PTFE were not accurate for this particular grade of PTFE.

Percent Mass Change

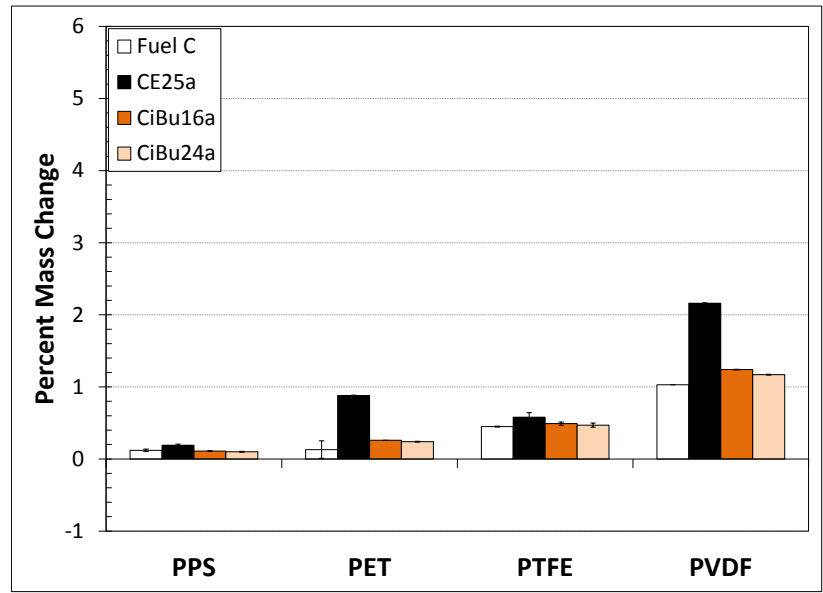

Percent Volume Change

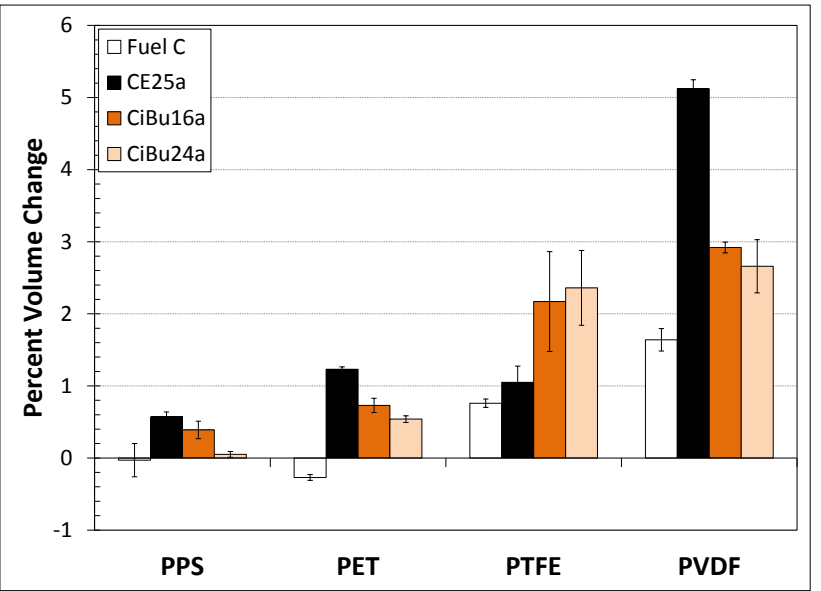

Fig. 47. Mass and volume change for plastic materials typically used as permeation barriers. Specimens were immersed in the test fuel liquids for 16 weeks at $60^{\circ} \mathrm{C}$.
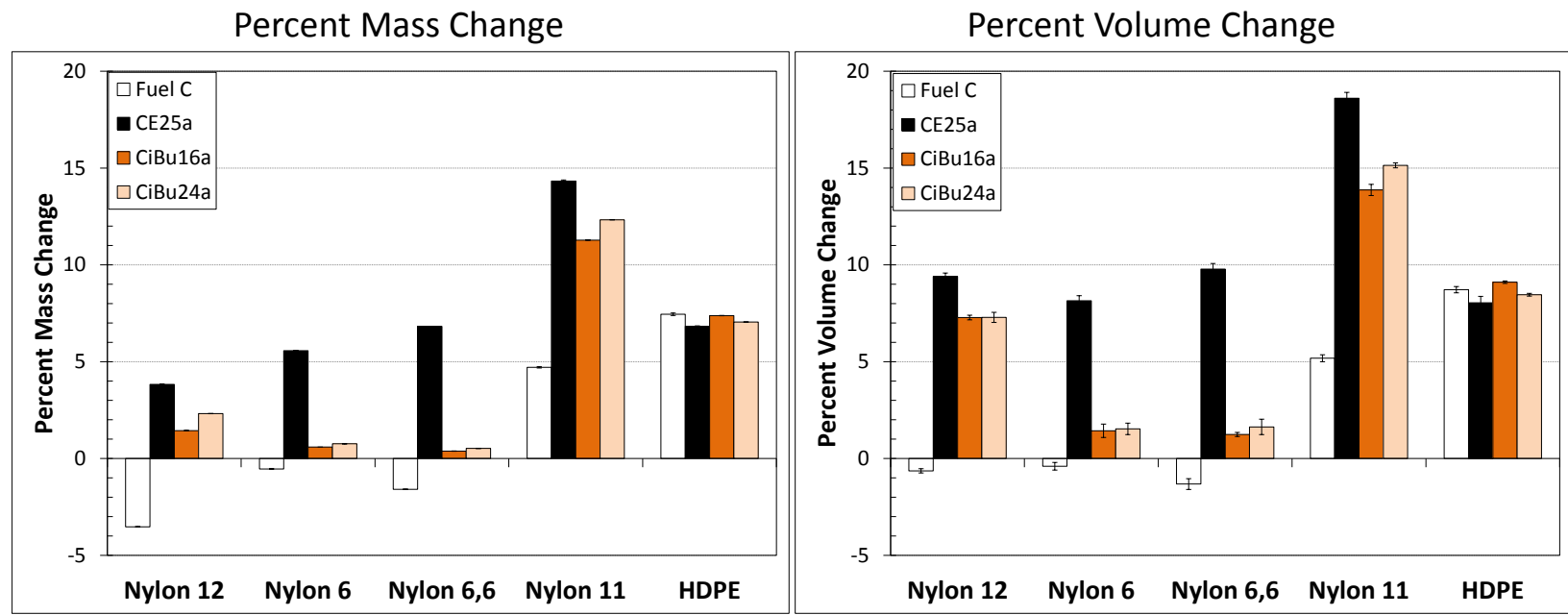

Fig. 48. Mass and volume change for nylons and HDPE. Specimens were immersed in the test fuel liquids for 16 weeks at $60^{\circ} \mathrm{C}$. 
Percent Mass Change

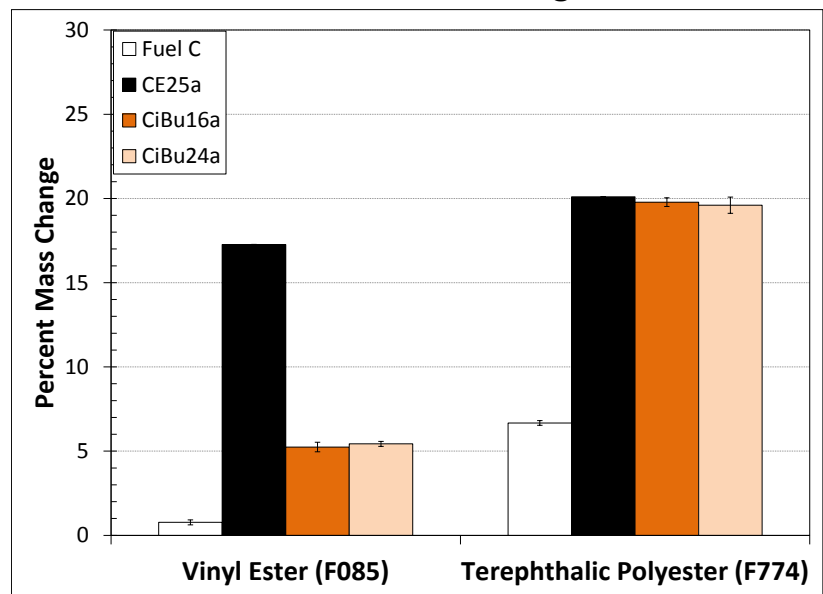

Percent Volume Change

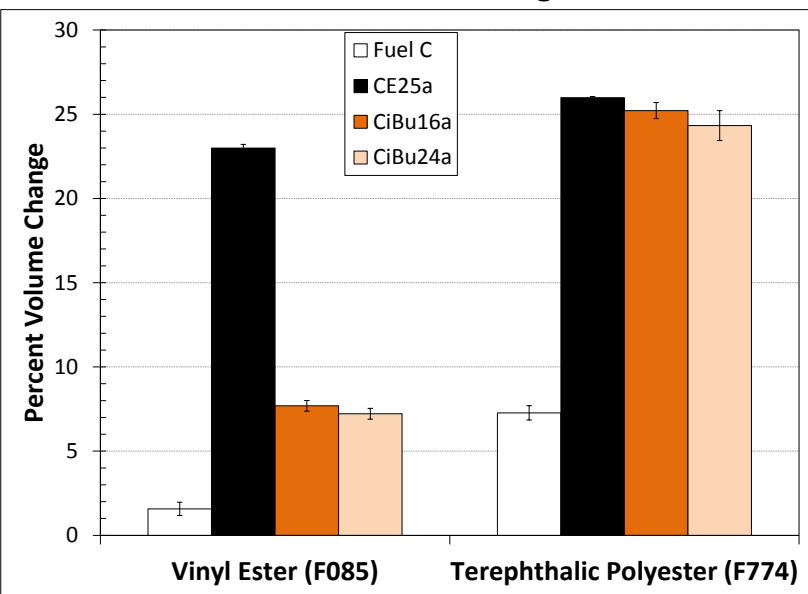

Fig. 49. Mass and volume change for two resin materials. Specimens were immersed in the test fuel liquids for 16 weeks at $60^{\circ} \mathrm{C}$.
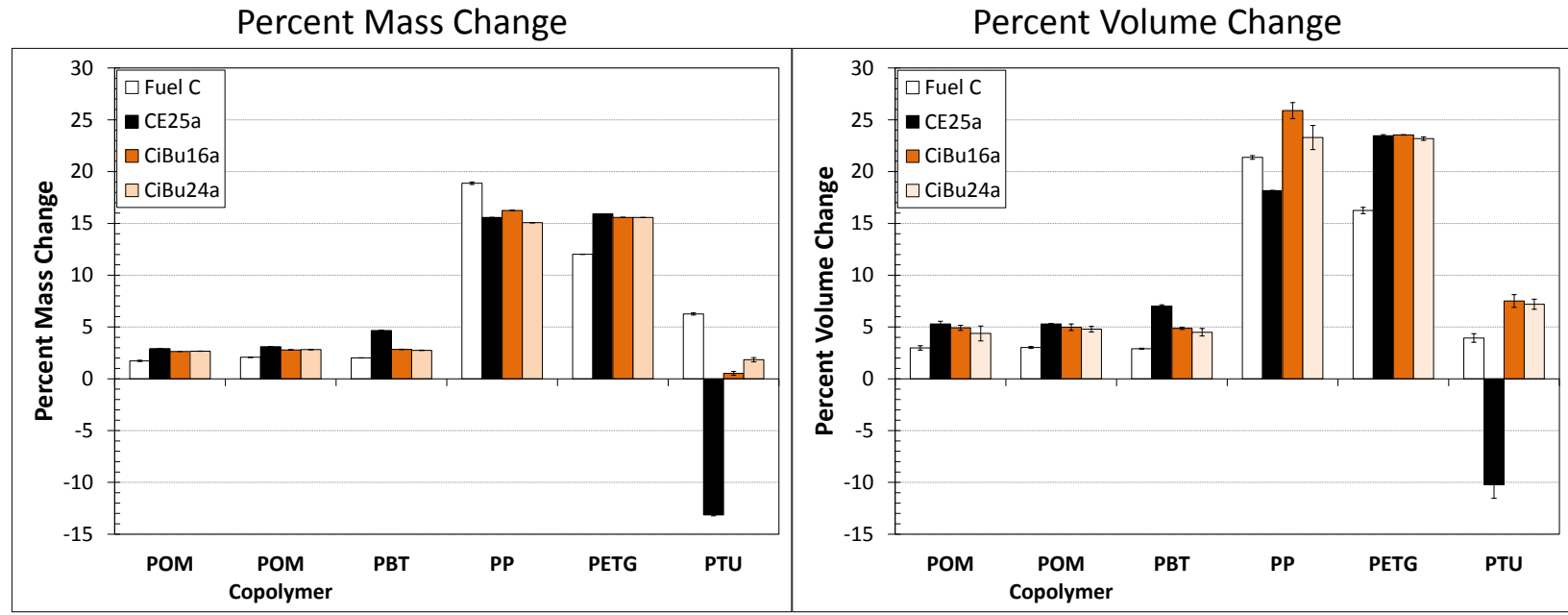

Fig. 50. Mass and volume change for other common plastic materials. Specimens were immersed in the test fuel liquids for 16 weeks at $60^{\circ} \mathrm{C}$.

Of the four barrier materials, PVDF exhibited the most swell with the test fuels, although the extent was quite low. The mass was raised $0.5 \%$ with exposure to each of the test fuels, but the volume varied according to fuel type. Fuel C produced a 1.5\% volume increase, and the additions of aggressive ethanol and isobutanol further increased swelling by 5 and 3\%, respectively.

\subsubsection{Nylons and HDPE}

The volume and mass changes for the nylon and HDPE specimens are shown in Fig. 48. The solubility curves (Figs. 18-22) predicted these materials to be insoluble with the test fuel formulations; however, these predictions were only partially accurate as shown in the figure. Nylons 6 and 6,6 exhibited similar behavior as they both demonstrated negligible swelling with Fuel C, CiBu16a, and CiBu24a, which is consistent with the solubility analysis. However, CE25a produced a 7.5 to $10 \%$ increase in swelling in these two nylon grades. The other petroleum-based nylon material was Nylon 12, which was also unaffected by Fuel C; however, the addition of 25\% aggressive ethanol caused its volume to expand over 9\%. CiBu16a and CiBu24a produced a slightly lower volume expansion (7\%). Bio-derived Nylon 11 
differed from the petro-nylons in that the volume expanded 5\% with exposure to Fuel C. Adding 25\% ethanol further expanded the volume to $18 \%$, while isobutanol produced a slightly lower $15 \%$ volume increase.

The mass and volume increases for HDPE were essentially the same for all test fuels, an indication that Fuel C was primarily responsible for this effect. The mass increase was around $7 \%$, and the volume was only slightly higher at around 8\%. These increases are small but significant; they do indicate that the addition of ethanol and isobutanol to gasoline will not further increase mass and volume.

\subsubsection{Fiberglass resins}

The results for the vinyl ester (Vipel F085) and terephthalic polyester (Vipel F774) resins are shown in Fig. 49; for both materials, the mass and volume increases were similar. Two isophthalic polyester resins were also tested but, although they were intact following exposure to Fuel C, they had partially dissolved and fractured with exposure to CE25a, CiBu16a, or CiBu24a, and therefore are not included in the figure. Solubility analysis predicted low to moderate swelling for these two materials, and that the vinyl ester resin would have better compatibility to the test fuels than the terephthalic resin. The results show reasonable correlation with the predicted solubility. However, the vinyl ester resin showed considerably higher swell with CE25a than with Fuel C or fuels containing isobutanol.

The swelling results for the vinyl ester resin correlate well with the solubility curve for Fuel $\mathrm{C}$ and the isobutanol test fuels. The vinyl ester resin showed negligible volume swell with Fuel C, and low swell (5\%) with the isobutanol test fuels. However, this resin type was highly sensitive to ethanol as shown by the 22\% volume expansion accompanying CE25a exposure. The terephthalic ester resin swelled $7 \%$ with Fuel C, while the alcohol additions pushed the volume swell to between 24 and 26\%. Unlike vinyl ester, the added isobutanol did not show improved compatibility over CE25a.

\subsubsection{Other common plastics}

In addition to the plastic types typically used in fuel containment and storage systems, this study also included other common mid-range and commodity plastics and their mass/volume change is shown in Fig. 50. As seen in the figure, the magnitude of the mass change was similar to (albeit slightly lower) than the accompanying change in volume. The mass/volume changes for the two acetals and PBT were around 2 and 5\%, respectively, and these results correspond to their predicted solubility. Fuel C increased the volume around $2.5 \%$ and an additional modest (2-3\%) increase was observed when these specimens were exposed to the test fuels containing ethanol or isobutanol. In each case the mass and volume swell for these materials was slightly higher with exposure to CE25a than for either CiBu16a or CiBu24a.

Moderate to high levels of swelling and mass increase were observed for PP and PETG for each test fuel, and this result is consistent with the prior solubility analysis. For PP most, if not all, of this increase can be attributed to Fuel C; this is especially true for the mass change, although isobutanol may add a small additional amount to the total volume. For PETG, Fuel $\mathrm{C}$ by itself increased the mass and volume by $12.5 \%$ and $16 \%$, respectively. The addition of either ethanol or isobutanol further increased the volume by another 7\%, and there was no noticeable difference in the magnitude of swell resulting from CE25a, CiBu16a, or CiBu24a.

Interestingly, PTU lost significant levels of mass and volume with exposure to CE25a, but CiBu16a and CiBu24a both produced a modest level of swelling ( 5\%), which was slightly higher than the level observed for Fuel C. Unlike ethanol, adding isobutanol to Fuel C did not cause a decrease in the mass/volume. Rather a modest increase in swelling was observed for these fuel types. 


\subsubsection{Rubber-impregnated cork}

The mass and volume changes for the two cork materials are shown in Fig. 51. Significant swelling was noted for both cork specimens. The results for cork (impregnated with NBR) were unusual in that the change in mass was considerably higher than the corresponding volume change. This effect is attributed to the cork fraction, since this behavior was not noted for any of the polymer materials. As shown in the figure, the cork/NBR gasket material was found to be highly sensitive to the presence of alcohol in the test fuels. Cork/NBR expanded a moderate amount ( 12\%) with Fuel C but swelled around $50 \%$ for the test fuels containing either ethanol or isobutanol. The cork/ECO specimens showed significant volume swell with the isobutanol test fuels; however, no comparison could be made with either Fuel C or CE25a.

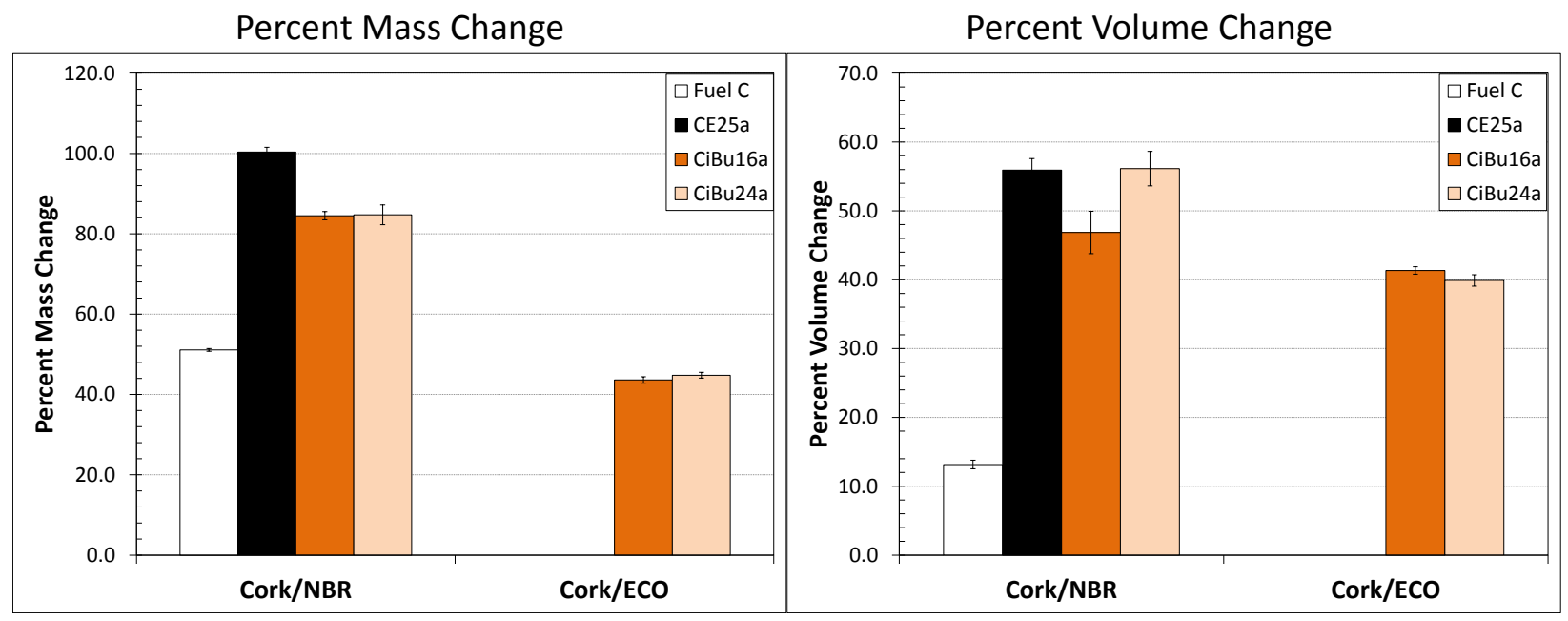

Fig. 51. Mass and volume change for rubberized cork materials. Specimens were immersed in the test fuel liquids for 16 weeks at $60^{\circ} \mathrm{C}$.

\subsubsection{Wet Hardness (or Softening)}

The plastics specimens were not observed to soften to the extent of the elastomers. The much higher density and molecular weight of these materials did not allow high levels of permeation (as seen in the elastomers) to occur.

\subsubsection{Permeation barrier plastics}

The point change in hardness results for the permeation barrier plastics is shown in Fig. 52 for the specimens while in the wetted state. Each of these materials exhibited essentially negligible change in hardness as demonstrated by the fact that the point changes are all within \pm 3 points from the original baseline condition. This result is not surprising since the original volume and mass changes (while in the wetted state) were low for these materials. 


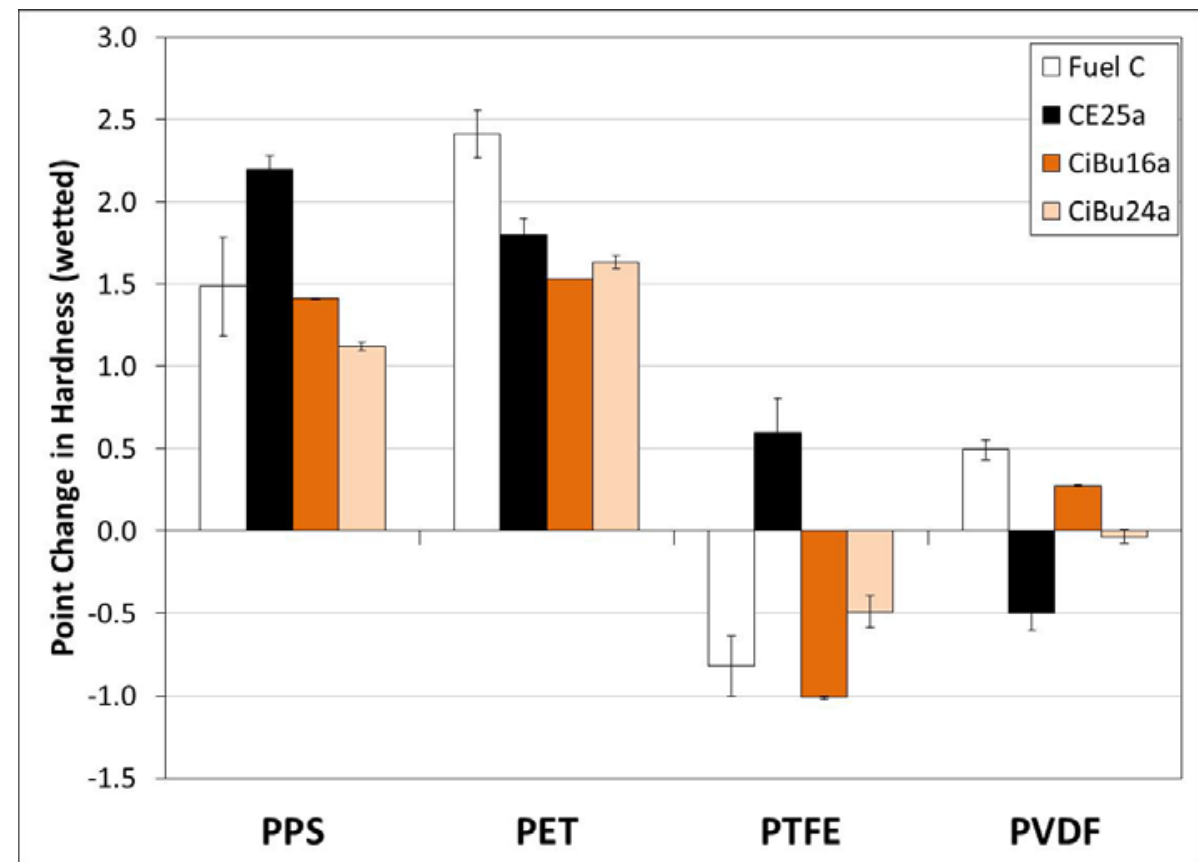

Fig. 52. Point change in hardness while in the wetted state for plastic materials typically used as permeation barriers. Specimens were immersed in the test fuel liquids for 16 weeks at $60^{\circ} \mathrm{C}$.

\subsubsection{Nylons and HDPE}

The point change in hardness for the nylons is shown in Fig. 53 along with HDPE. The results varied according to nylon type.

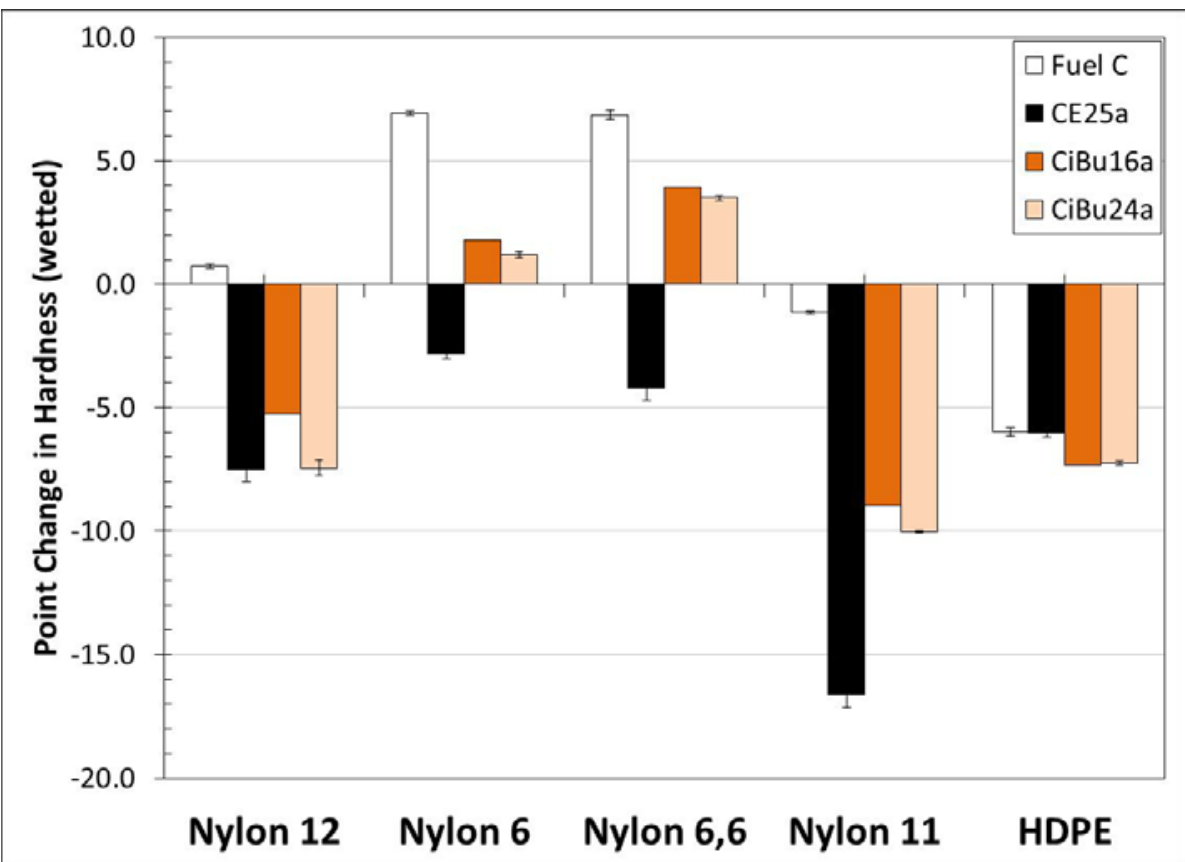

Fig. 53. Point change in hardness while in the wetted state for nylons and HDPE. Specimens were immersed in the test fuel liquids for 16 weeks at $60^{\circ} \mathrm{C}$. 
Nylon 12 hardness was unaffected by Fuel C, but was slightly softened 5 to 7 points with exposure to the test fuels containing ethanol or isobutanol. Nylons 6 and 6,6 exhibited similar behavior with ethanol, but exhibited a 7 point increase with exposure to fuel C. These two nylons exhibited a lower amount of hardening for the fuels containing isobutanol, and they were both slightly softened when exposed to CE25a. Nylon 11, which was bio-based, exhibited negligible change for Fuel C; however, the added ethanol and isobutanol dropped the hardness by 17 and 10 points, respectively (a substantial degree of softening). HDPE showed a small, but consistent, level of softening for each test fuel formulation, an implication that Fuel C is primarily responsible.

\subsubsection{Fiberglass resins}

The results for the fiberglass resins are presented in Fig. 54. Both resin types experienced hardness reductions in proportion to their level of volume swell. Softening for the vinyl polyester was essentially negligible with Fuel $\mathrm{C}$ and the isobutanol test fuels, but a significant hardness decrease occurred with exposure to CE25a. The terephthalic ester resin was slightly softened by Fuel C, but the alcohol additions significantly added to the level of observed softening.

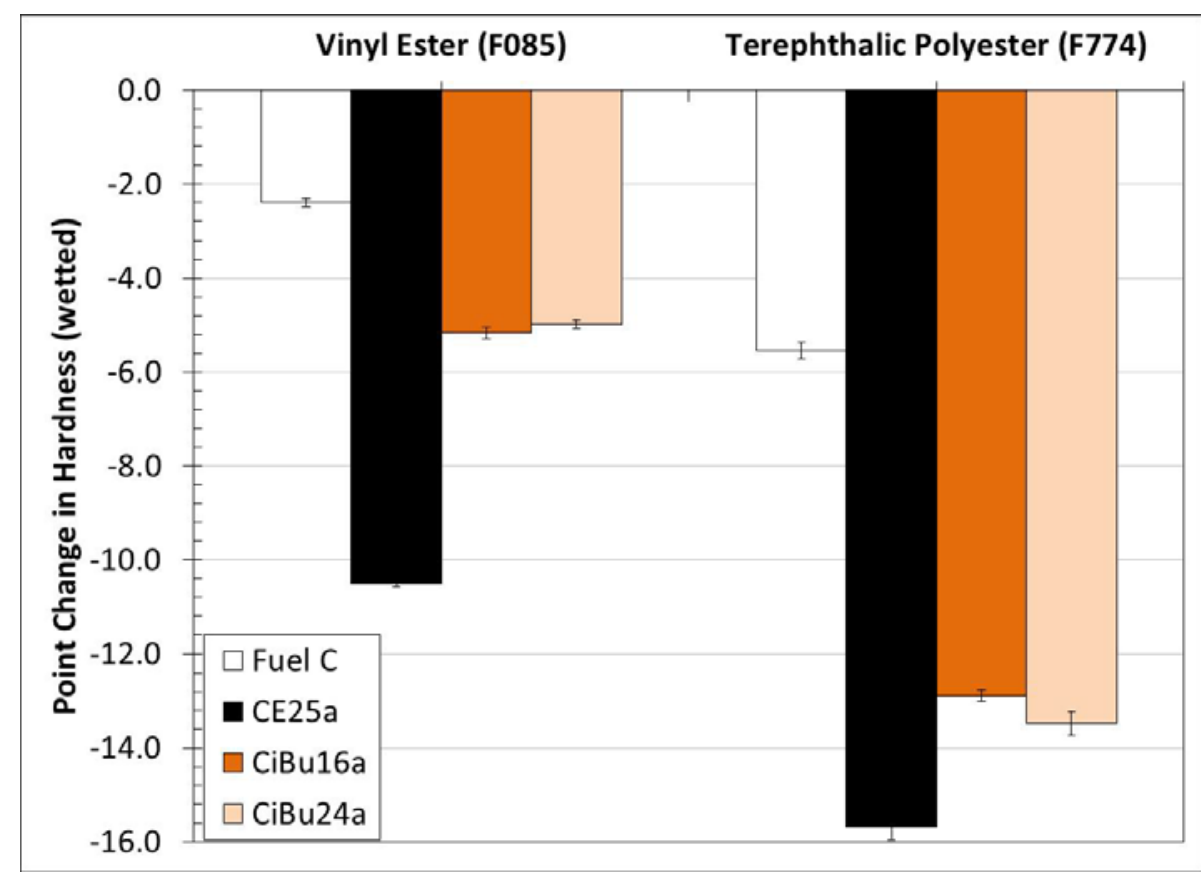

Fig. 54. Point change in hardness while in the wetted state for two fiberglass resin materials. Specimens were immersed in the test fuel liquids for 16 weeks at $60^{\circ} \mathrm{C}$.

\subsubsection{Other common plastics}

The wetted hardness results for mid-range and commodity plastics are shown in Fig. 55. POM and the POM copolymer both exhibited negligible softening with exposure to the test fuels containing one of the two types of alcohols. Fuel $\mathrm{C}$ did not affect wetted hardness for POM. The hardness for PBT was lowered slightly by Fuel $\mathrm{C}$, and the hardness dropped a total of 4-5 points for the test fuels containing either ethanol or isobutanol. This hardness decrease is considered small. 


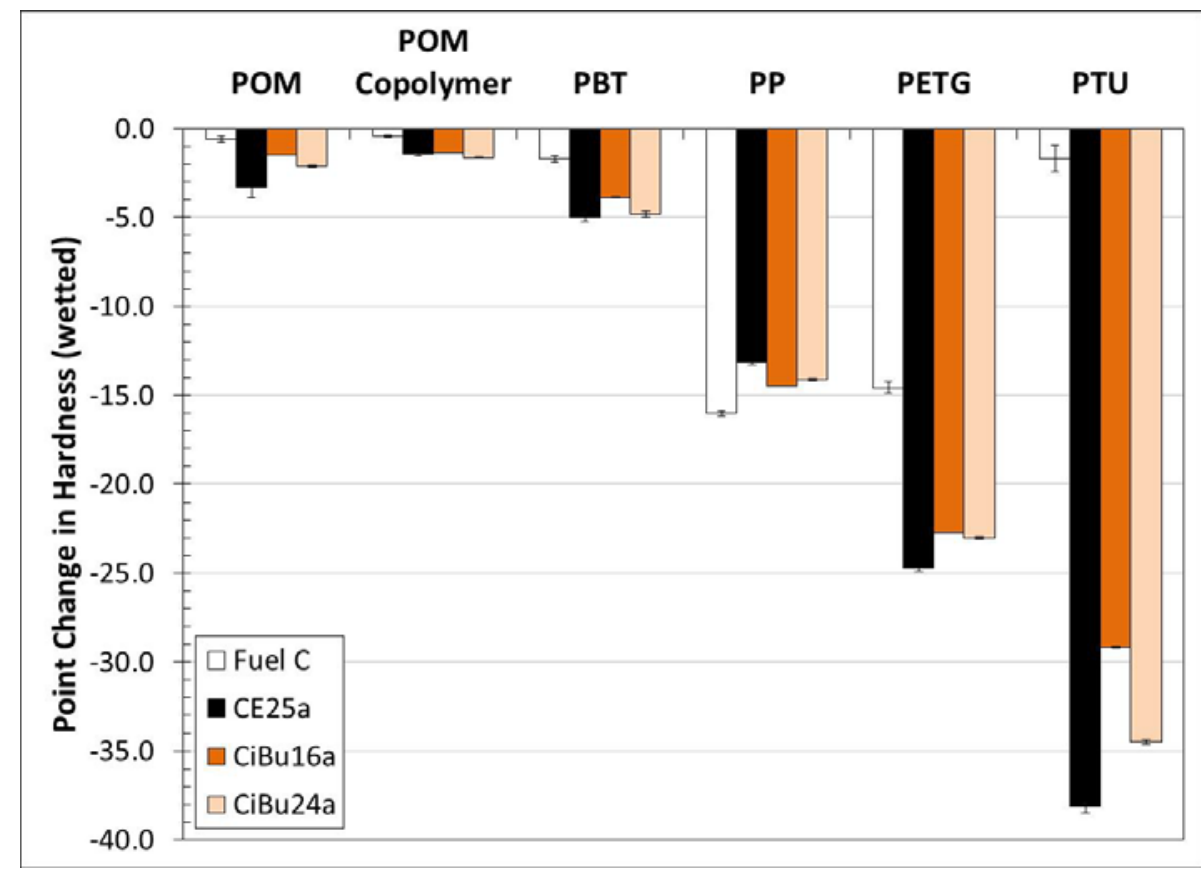

Fig. 55. Point change in hardness while in the wetted state for other common plastic materials. Specimens were immersed in the test fuel liquids for 16 weeks at $60^{\circ} \mathrm{C}$.

Polypropylene was observed to significantly soften with exposure to the test fuels. Its hardness was lowered by 13-15 points with exposures to the test fuels. Most of this drop in hardness is likely due to Fuel C, with little observable contribution from the alcohol additions. PETG is a mix of PET and an unknown co-polymer material. In contrast to PET (Fig. 47), PETG was dramatically softened by the test fuels. Fuel C was responsible for a 15 point decrease in hardness (from baseline), and the added alcohols were observed to decrease hardness another 7-10 points. Clearly, the co-polymer had a significant (if not dominant) effect on the hardness result for this particular PET-based material.

As shown in the figure, PTU was the plastic most affected by the addition of alcohol. Fuel C did not affect PTU hardness significantly. However, ethanol and isobutanol reduced the hardness significantly by around 30 to 40 points. Of the two alcohol blended fuel types, CE25a decreased the hardness more than the isobutanol blends.

\subsubsection{Rubber-impregnated cork}

The cork specimens (as shown in Fig. 56) exhibited significant softening with exposure to the test fuels containing either ethanol or isobutanol. For cork (impregnated with NBR) the swelling associated with Fuel $\mathrm{C}$ was relatively low ( 5\%), but for CE25a, CiBu16a, and CiBu24a, the hardness declined by over 30 points, which is considered high. The cork sample containing ECO was also notably softened by CiBu16a and CiBu24a, but the point drop was only half that observed for the cork/NBR specimens. 


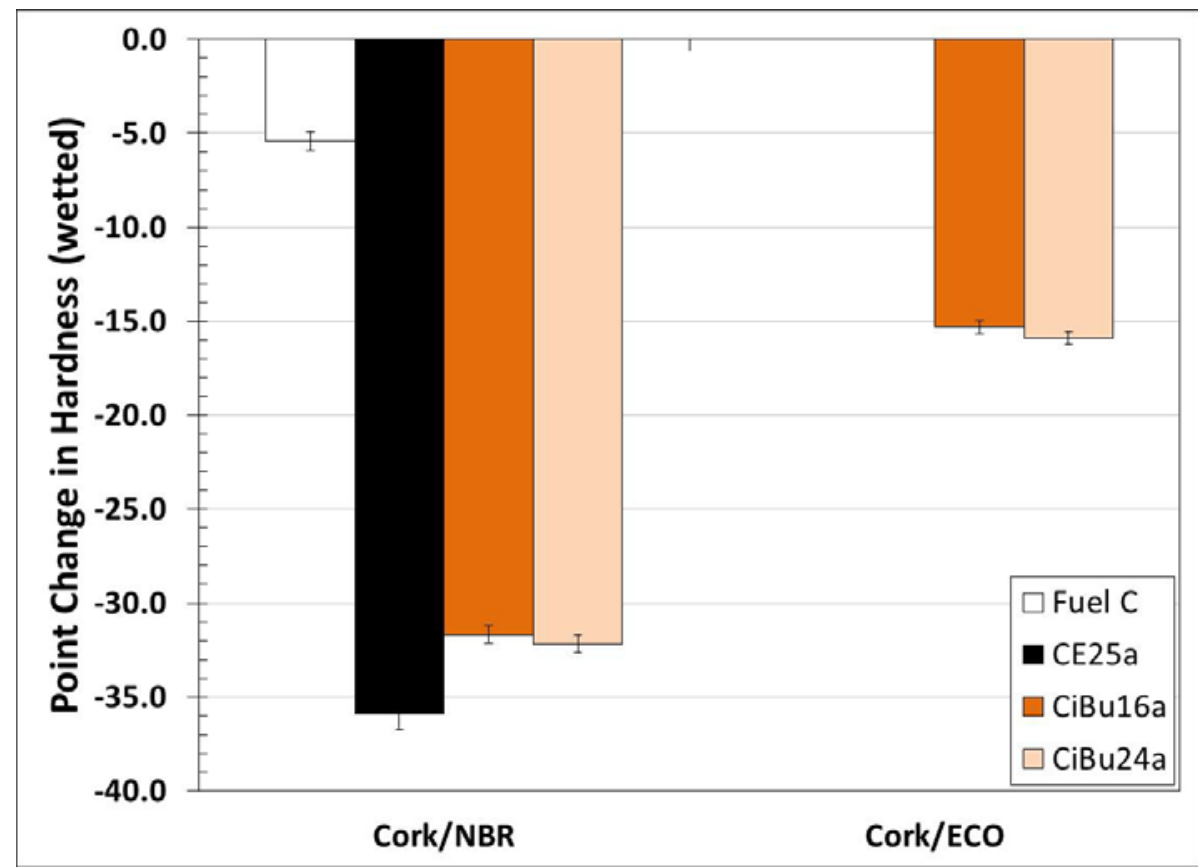

Fig. 56. Point change in hardness while in the wetted state for cork materials. Specimens were immersed in the test fuel liquids for 16 weeks at $60^{\circ} \mathrm{C}$.

\subsubsection{Dried Mass and Volume for Wetted Specimens}

In general the mass and volume changes for the plastics after being dried were less than the values obtained for the elastomers. For most plastics the dried mass and volume either returned near the original baseline value or they experienced a small increase associated with the absorbed liquids. Unlike many elastomers, plastics are not normally compounded with highly extractable plasticizers, such as phthalates, and therefore do not shrink as much when dried. There were, however, two notable exceptions: both Nylon 12 and PTU lost mass (and hence volume) after being dried. The mass loss accompanying Nylon 12 was strongly associated the Fuel C, whereas for PTU, it was the aggressive alcohol additives that caused the decrease in mass and volume.

\subsubsection{Permeation barrier plastics}

As shown in Fig. 57, the four plastics known to be used as permeation barriers exhibited slight mass increases with the test fuels. The volume increase was also small. For PPS, PET, and PVDF, the relative volume increase tracked with the relative mass increase. However, for PTFE, the Fuel C formulation produced the highest mass increase and the lowest volume expansion of the test fuels. Both PET and PVDF incurred noticeably higher mass and volume gains with CE25a than with the other test fuels. 
Percent Mass Change

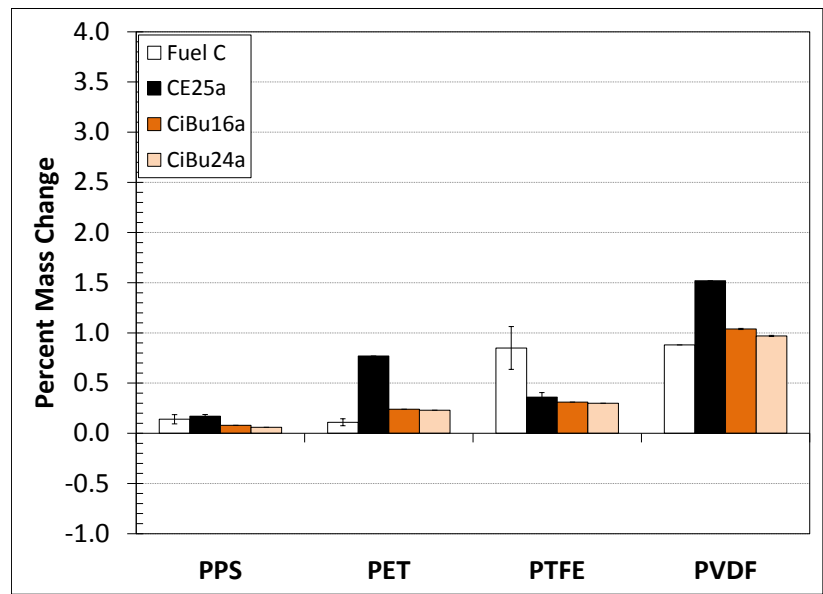

Percent Volume Change

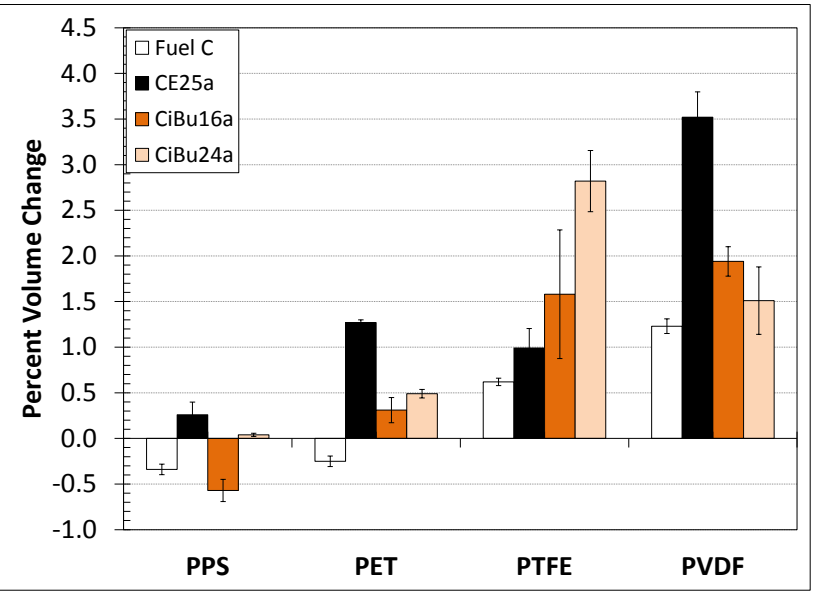

Fig. 57. Dried mass and volume change for permeation barrier plastics. Specimens were immersed in the test fuel liquids for 16 weeks at $60^{\circ} \mathrm{C}$ and then dried at $60^{\circ} \mathrm{C}$ for 65 hours.

\subsubsection{Nylons and HDPE}

The dried mass and volume change (from baseline) are shown in Fig. 58 for the four nylons and HDPE. HDPE essentially returned to its original mass and volume after being dried. The nylon results varied according to fuel formulation and type of nylon. Consistent with other measured properties, Nylon 6 and Nylon 6,6 performed similarly. For both materials, the mass and volume returned to their original values after being exposed to Fuel $\mathrm{C}$ and the fuels containing isobutanol. In marked contrast, 25\% aggressive ethanol was observed to produce a small increase in mass and volume. Nylon 12 was unusual in that it lost significant mass and volume after being dried. Most of this loss can be attributed to Fuel C, but ethanol produced a small additional contribution. The dried Nylon 11 specimens showed a small (4\%) increase in the mass and volume following exposure to Fuel $\mathrm{C}$, and the isobutanol fuels raised the mass to between 5 and $6 \%$ and the volume to around (8\%). For this material, ethanol produced the lowest property change $(\sim 2 \%)$ from the original baseline condition.

Percent Mass Change

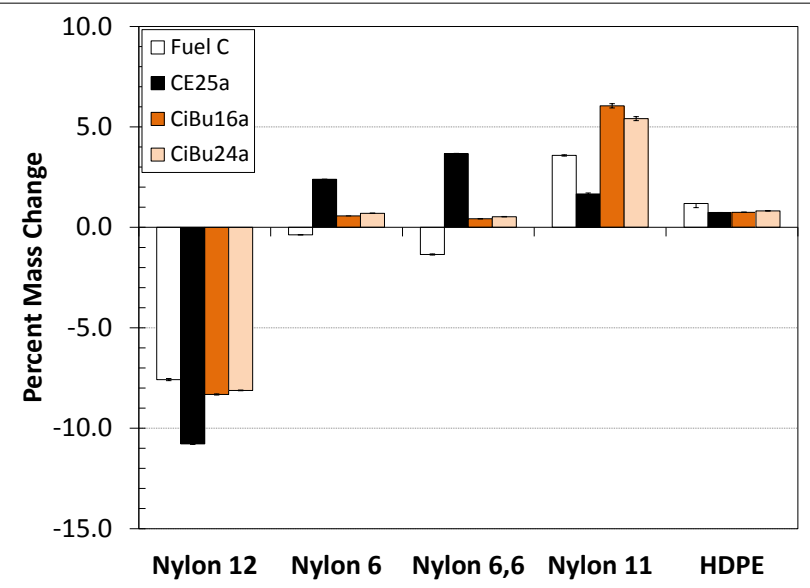

Percent Volume Change

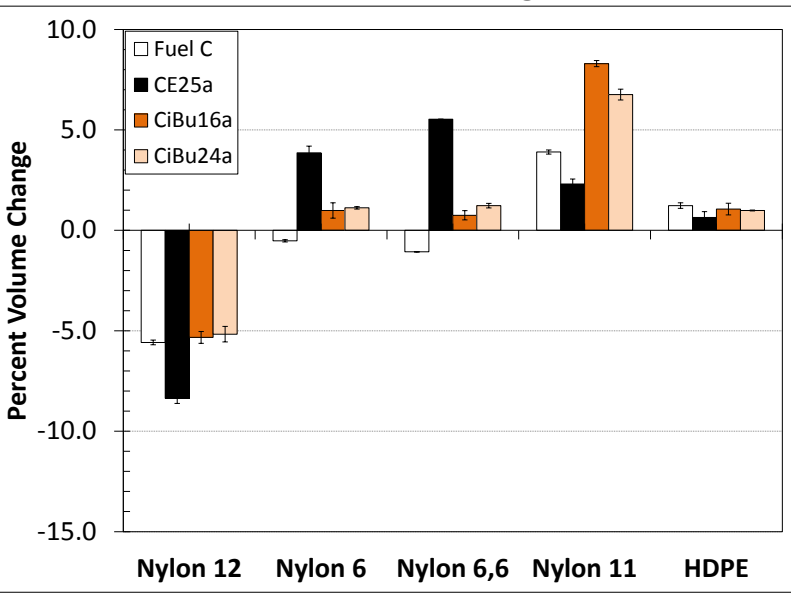

Fig. 58. Dried mass and volume change for nylons and HDPE. Specimens were immersed in the test fuel liquids for 16 weeks at $60^{\circ} \mathrm{C}$ and then dried at $60^{\circ} \mathrm{C}$ for 65 hours. 


\subsubsection{Fiberglass resins}

The mass and volume change for the two fiberglass resins after drying was significant, as shown in Fig. 59. The terephthalic polyester resin dried mass and volume were $5 \%$ higher than the starting condition following exposure to Fuel C. The addition of $25 \%$ ethanol and $24 \%$ isobutanol increased the dried volume to around 12 and 17\%, respectively. After drying, the vinyl ester resin returned near the original condition when exposed to Fuel C. The addition of $25 \%$ ethanol had a pronounced effect by raising the dried mass by $10 \%$ and the starting volume by $13 \%$. For this resin material, isobutanol increased the mass and volume to around 5\%, which roughly matches the swell observed for terephthalic polyester resin exposed to Fuel C. The observed increase in mass and volume indicate retention of test fuels (or one of their components) in the plastic structure.

Percent Mass Change

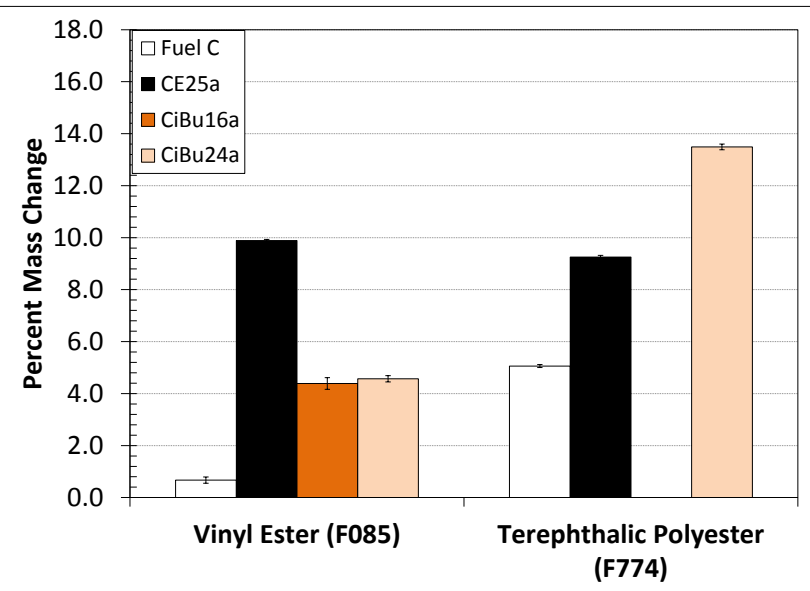

Percent Volume Change

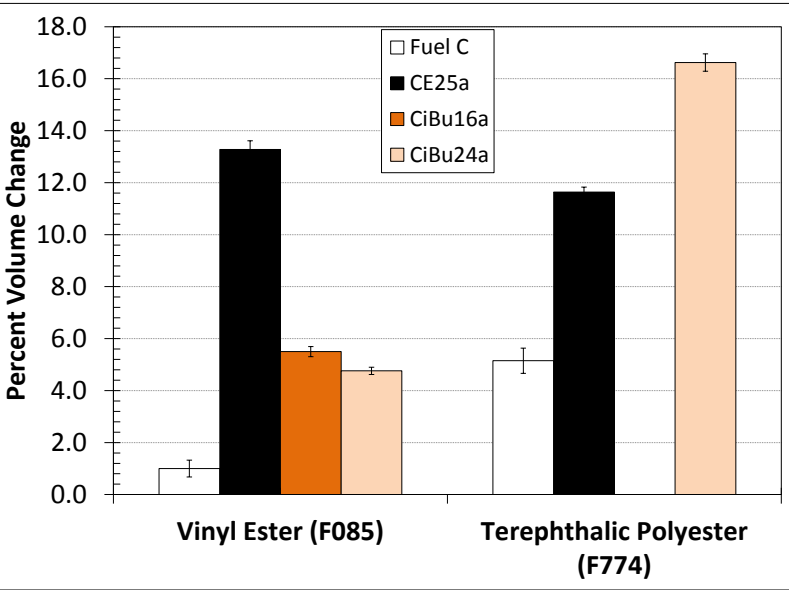

Fig. 59. Dried mass and volume change for two fiberglass resin materials. Specimens were immersed in the test fuel liquids for 16 weeks at $60^{\circ} \mathrm{C}$ and then dried at $60^{\circ} \mathrm{C}$ for 65 hours.

\subsubsection{Other common plastics}

As shown in Fig. 60, the acetals (POM and POM copolymer) and PP exhibited only a slight increase in mass and volume (over the baseline condition) following dry-out. PBT showed a small but significant gain in mass and volume, while PETG experienced a large 10\% increase in both properties. This large mass and volume increase is similar to that observed for the fiberglass resins exposed to the test fuels containing alcohol. This fluid retention also appears to be stable and possibly permanent. The dried mass and volume of PTU was not affected by Fuel C, but both of these properties exhibited a significant decrease in mass and volume when exposed to CE25a, CiBu16a and CiBu24a. (The dried volume for the PTU specimen exposed to CE25a was not made since this specimen had fractured.) The extent of the mass and volume reduction following drying strongly indicates that PTU may not be considered compatible with the test fuels containing added ethanol or isobutanol. 


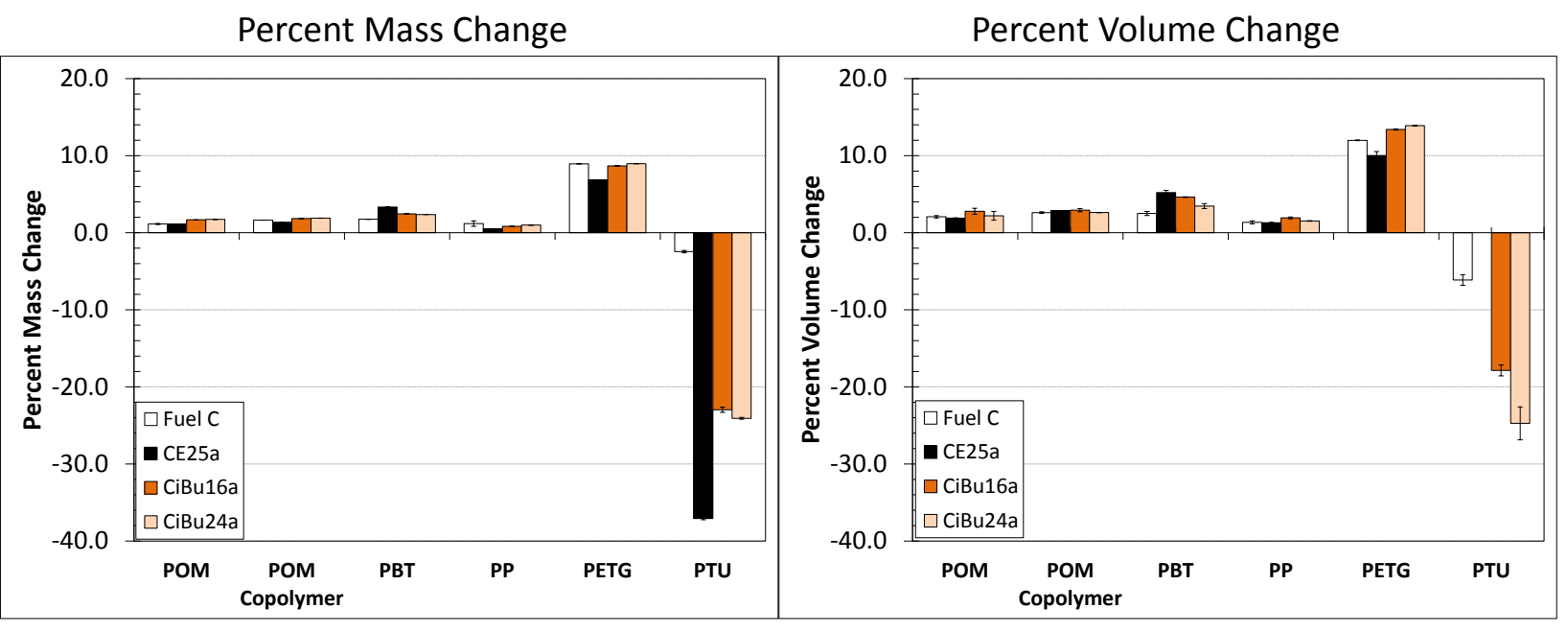

Fig. 60. Dried mass and volume change for other common plastic materials. Specimens were immersed in the test fuel liquids for 16 weeks at $60^{\circ} \mathrm{C}$ and then dried at $60^{\circ} \mathrm{C}$ for 65 hours.

\subsubsection{Rubber-impregnated cork}

The two cork specimens were included along with the plastic materials for the 16-week exposures. These materials underwent significant shrinkage and mass loss when dried as shown in Fig. 61. Fuel C caused the cork/NBR specimens to lose $10 \%$ of the original mass and $12 \%$ of the volume. The test fuels containing alcohols caused a $15 \%$ mass reduction for this material. The addition of the $25 \%$ aggressive ethanol increased the shrinkage to $25 \%$ and isobutanol additions caused $20 \%$ shrinkage. Fuel C and CE25a were not evaluated for compatibility with cork/ECO, but it was noted that the isobutanol test fuels caused 10\% mass loss and a corresponding 15\% shrinkage. The rubber content for both materials was not known, therefore it is impossible to assess whether the mass loss was primarily caused by the rubber or cork fraction.
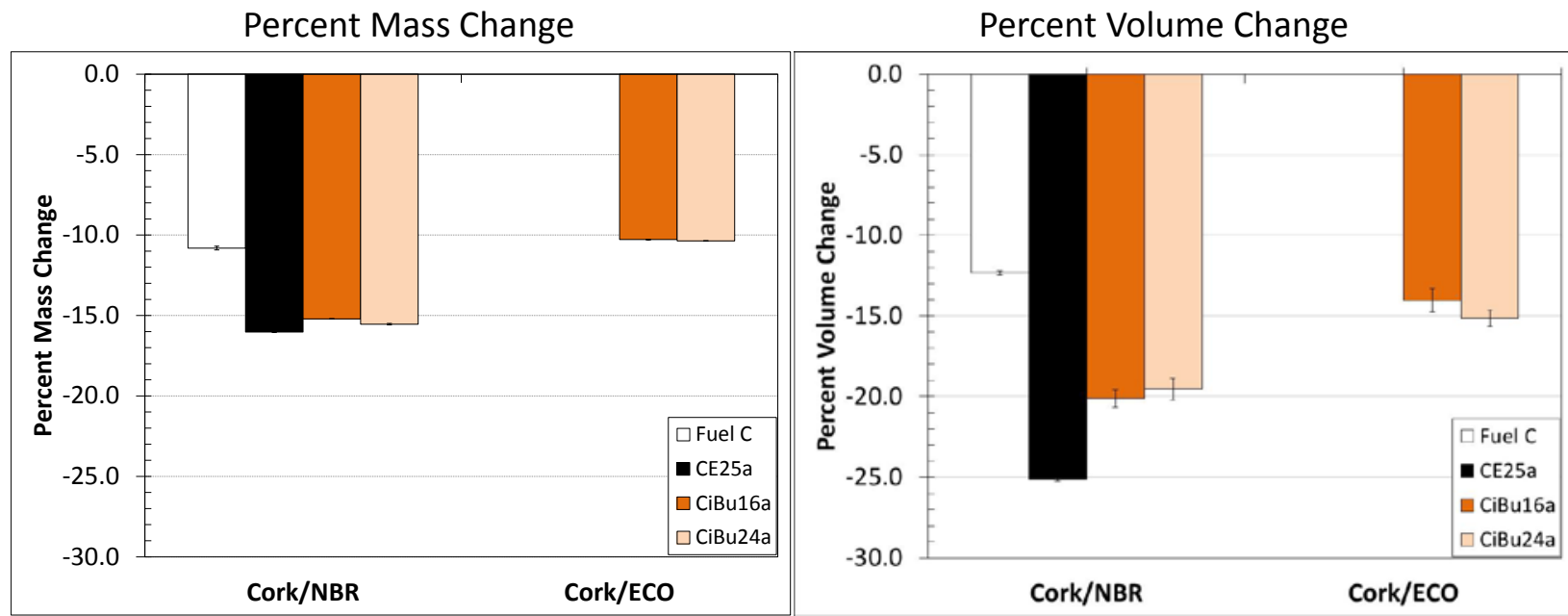

Fig. 61. Dried mass and volume change or two rubber-impregnated cork materials. Specimens were immersed in the test fuel liquids for 16 weeks at $60^{\circ} \mathrm{C}$ and then dried at $60^{\circ} \mathrm{C}$ for 65 hours. 


\subsubsection{Hardness Change Following Drying}

The hardness following drying was measured and the change from the original baseline value is shown in Figs. 62-65 for the plastics exposed to the liquid- and vapor-phase regions. In general the hardness change results were similar for the liquid and vapor exposures. The dried mass and volume also tended to correlate with hardness for those specimens that experienced a mass and volume increase after drying. Since this mass/volume increase is associated with fuel retention, the corresponding hardness was observed to decrease. Materials exhibiting a swell and softening in the dried state include the fiberglass resins, Nylon 11, and PETG.

Liquid Exposures

Vapor Exposures

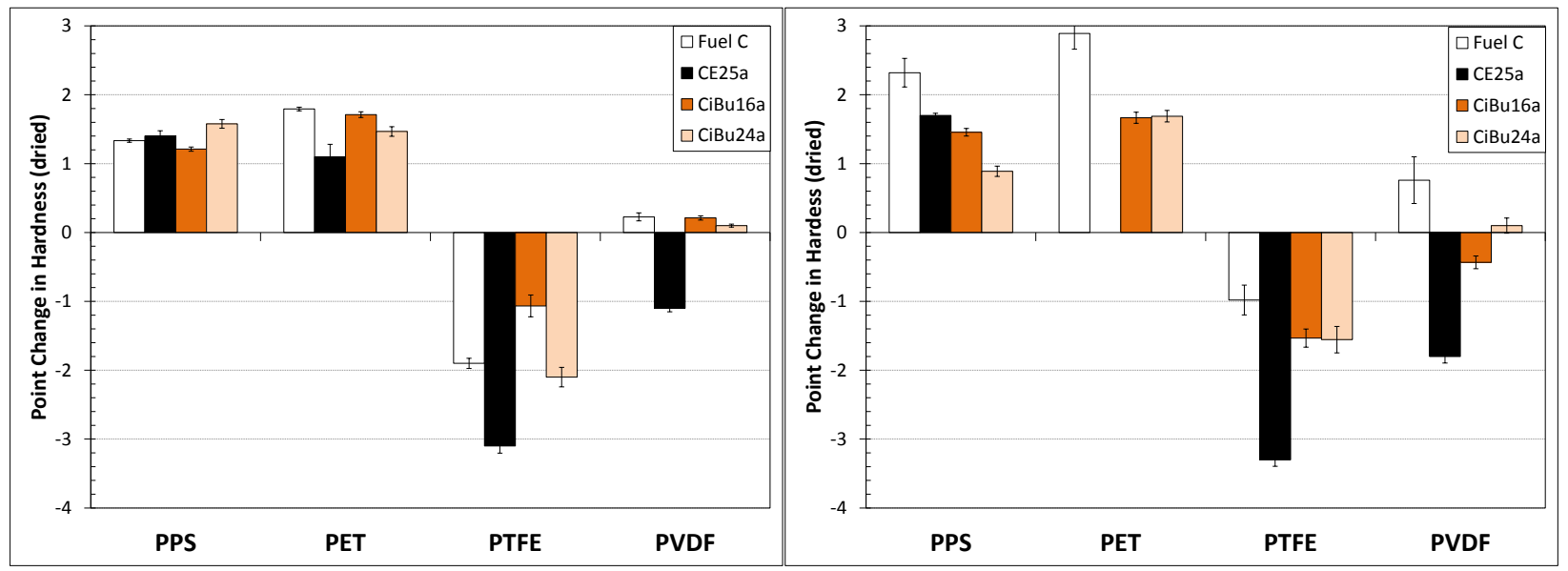

Fig. 62. Point change in hardness (after drying) for plastic materials typically used as permeation barriers.

Liquid Exposures

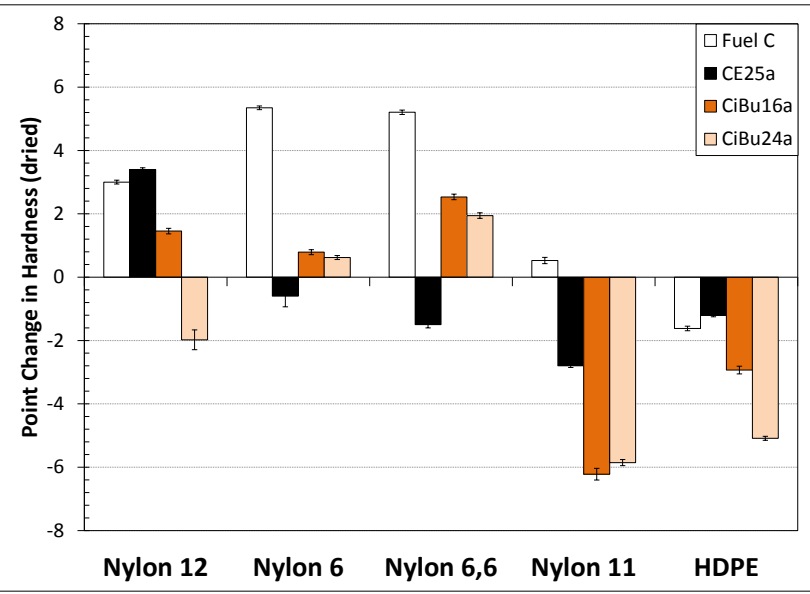

Vapor Exposures

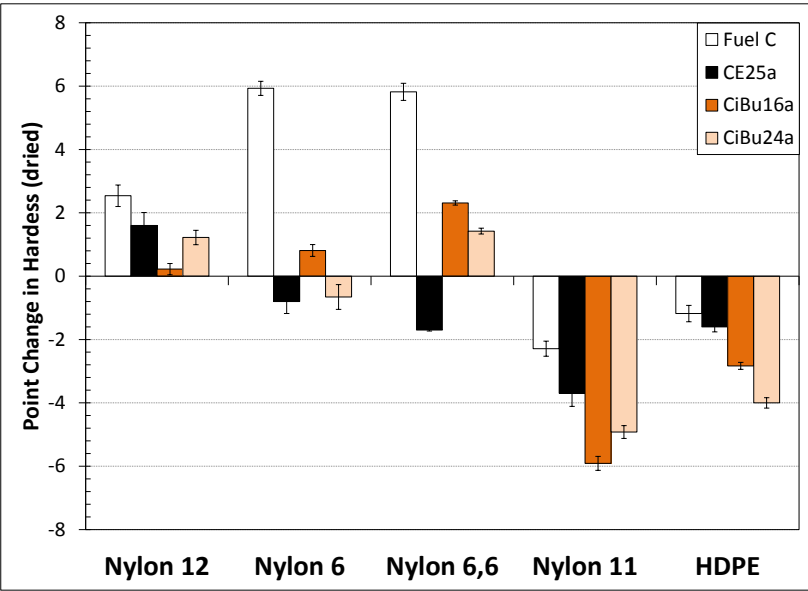

Fig. 63. Point change in hardness (after drying) for the nylon materials and HDPE. 

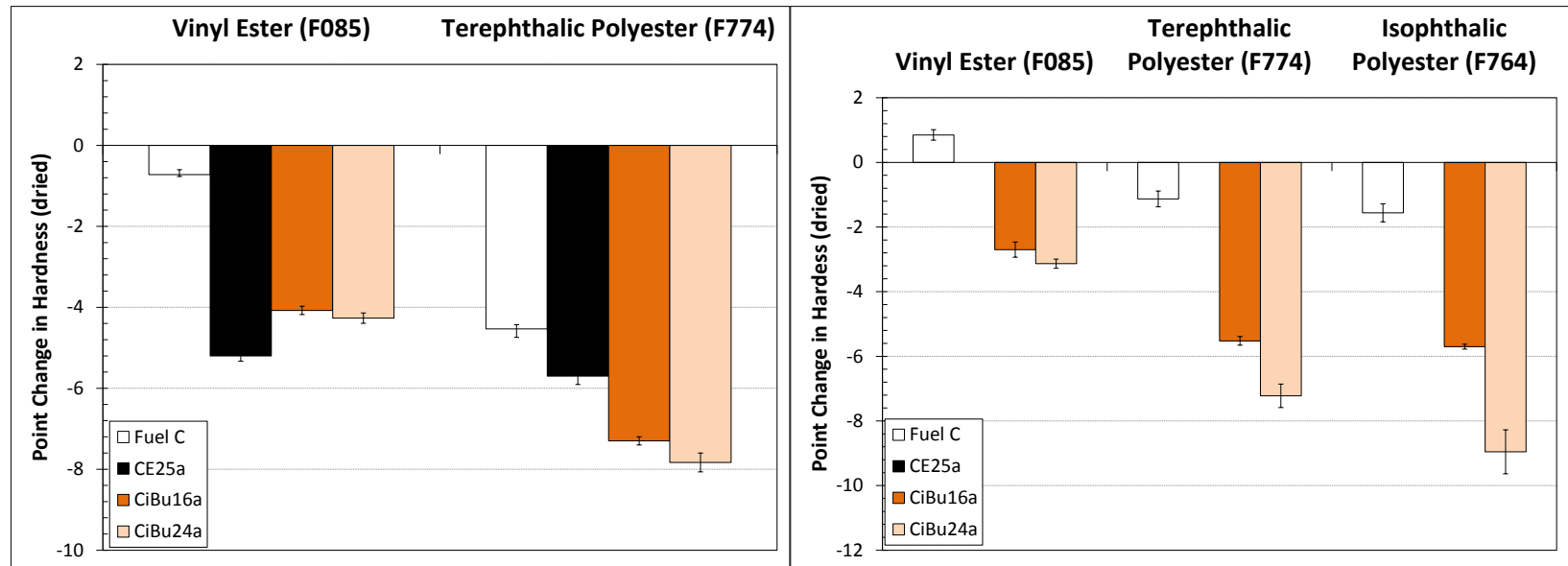

Fig. 64. Point change in hardness (after drying) for the resin materials.

Liquid Exposures

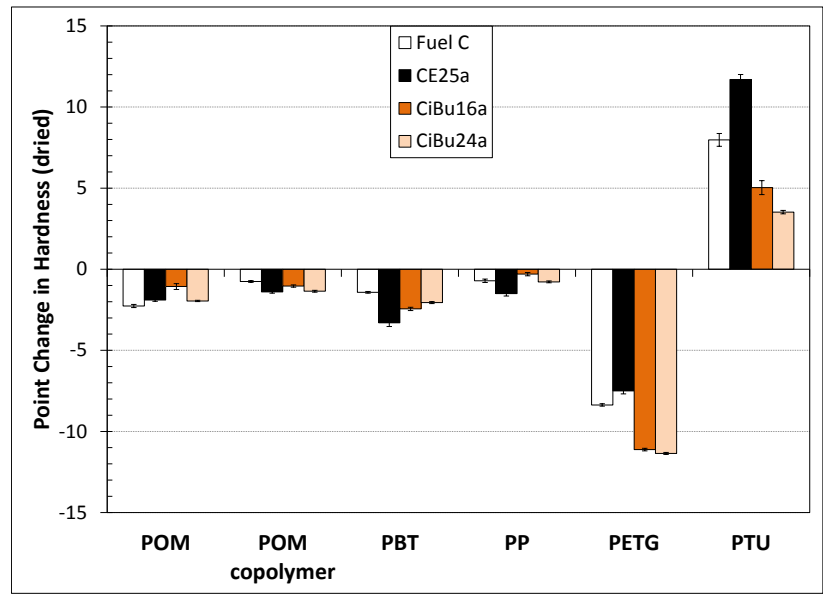

Vapor Exposures

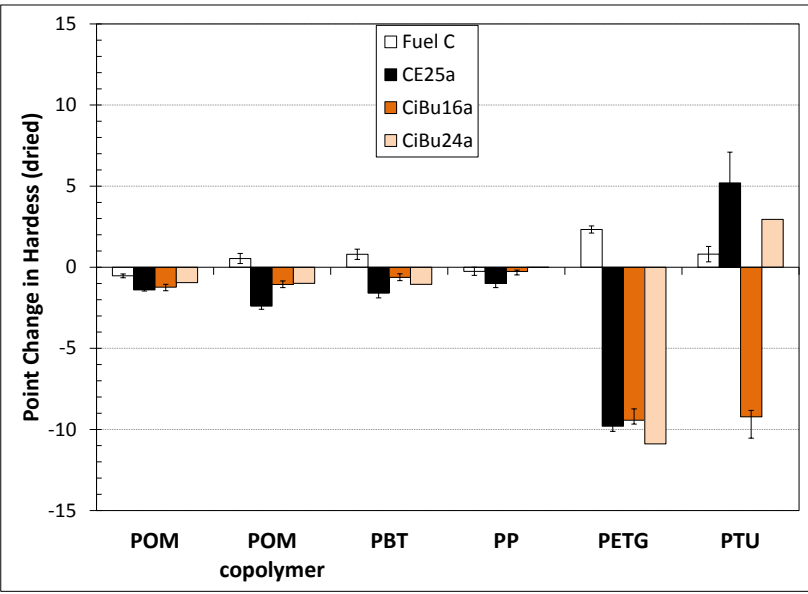

Fig. 65. Point change in hardness (after drying) for other common plastic materials.

\subsubsection{Permeation barrier plastics}

For the permeation barrier plastics the change in hardness results (see Fig. 62) were very low and are not considered significant. CE25a did cause a slight additional softening in PTFE and PVDF, but the change in hardness did not appear to be affected by the addition of isobutanol.

\subsubsection{Nylons and HDPE}

The nylons and HDPE showed slight changes in hardness following dry-out as shown in Fig. 63. Interestingly, although the extent of hardness change was extremely low, it was consistent for both vapor and liquid exposures. Of the nylons evaluated in this study, the petroleum-based nylons showed a slight hardness increase with exposure to Fuel C. In contrast, the bio-based Nylon 11 exhibited a slight decrease. Of the petroleum-based nylons shown in Fig. 10, Nylon 12 showed a slight increase in hardness after being exposed to Fuel $\mathrm{C}$ and CE25a, while isobutanol did not change the hardness significantly from the baseline value. Nylons 6 and 6,6 showed a small increase in hardness with the exposure to Fuel C, but the hardness results were less affected by the addition of either ethanol or isobutanol. Nylon 11 showed 
the most sensitivity to the added alcohols. It was not significantly influenced by Fuel C, but CE25a and the isobutanol blends did result in a small level of softening. HDPE exhibited similar performance to either liquid or vapor exposure. Fuel $\mathrm{C}$ caused a slight decrease in the hardness, which was further softened (albeit) slightly by and ethanol and slightly more so by isobutanol.

\subsubsection{Fiberglass resins}

The dry-out hardness results for the resins is shown in Fig. 64. Liquid and vapor results for the resin materials cannot be compared with ethanol, since no vapor specimens were included during the CE25a exposures. One of the isophthalic resins was able to survive vapor exposures in Fuel C, CiBu16a, and CiBu24a, and its results are included alongside the vinyl ester and terephthalic polyester resins. All of the resins that survived exposure remained in a slightly softened state after drying and the vinyl ester was observed to soften the least amount. The terephthalic polyester resin showed a small drop in hardness with exposure to Fuel C, CE25a produced a slightly higher drop in hardness and the isobutanol blended fuels caused the hardness to drop by 7-8 points. For each resin the Fuel $\mathrm{C}$ vapors did not affect the dried hardness, but they were softened by a small amount when exposed to CiBu16a and CiBu24a.

\subsubsection{Other common plastics}

The results for the remaining plastic materials are shown in Fig. 65. The change in hardness values for POM, POM copolymer, PBT and PP are considered negligible. However, small (but notable) softening occurred for PETG, which is a copolymer of PET. This softening at first appeared to be primarily caused by Fuel $\mathrm{C}$ for liquid exposures, but the hardness of the specimen exposed to the vapor phase was not affected. In contrast, the specimens exposed to the vapor-phase region of the test fuels containing ethanol or isobutanol were softened to roughly the same extent as exposure for the liquid test fuels (including Fuel C). This result indicates that the alcohol additions did, in fact, produce softening, but their influence was masked by Fuel $\mathrm{C}$ when in the liquid phase. In contrast to the other materials, the hardness for PTU was increased by exposure to the test fuels liquids, Fuel $\mathrm{C}$ has a noticeable effect, but CE25a caused additional embrittlement. CiBu16a and CiBu24a produced a smaller hardness increase than either Fuel C or CE25a. The vapor-phase results for PTU did not correspond to liquid-phase results. CE25a produced a small level of embrittlement as did CiBu24a, but CiBu16a caused measureable softening to occur.

\subsubsection{Rubber-impregnated cork}

The two cork specimens exhibited small changes in hardness after being dried as shown in Fig. 66. Fuel C did cause significant embrittlement in cork (impregnated with NBR), while the other test fuels only marginally changed the hardness from baseline. CE25a was observed to cause slightly higher embrittlement to occur than the test fuels containing isobutanol for both liquid and vapor exposures. 


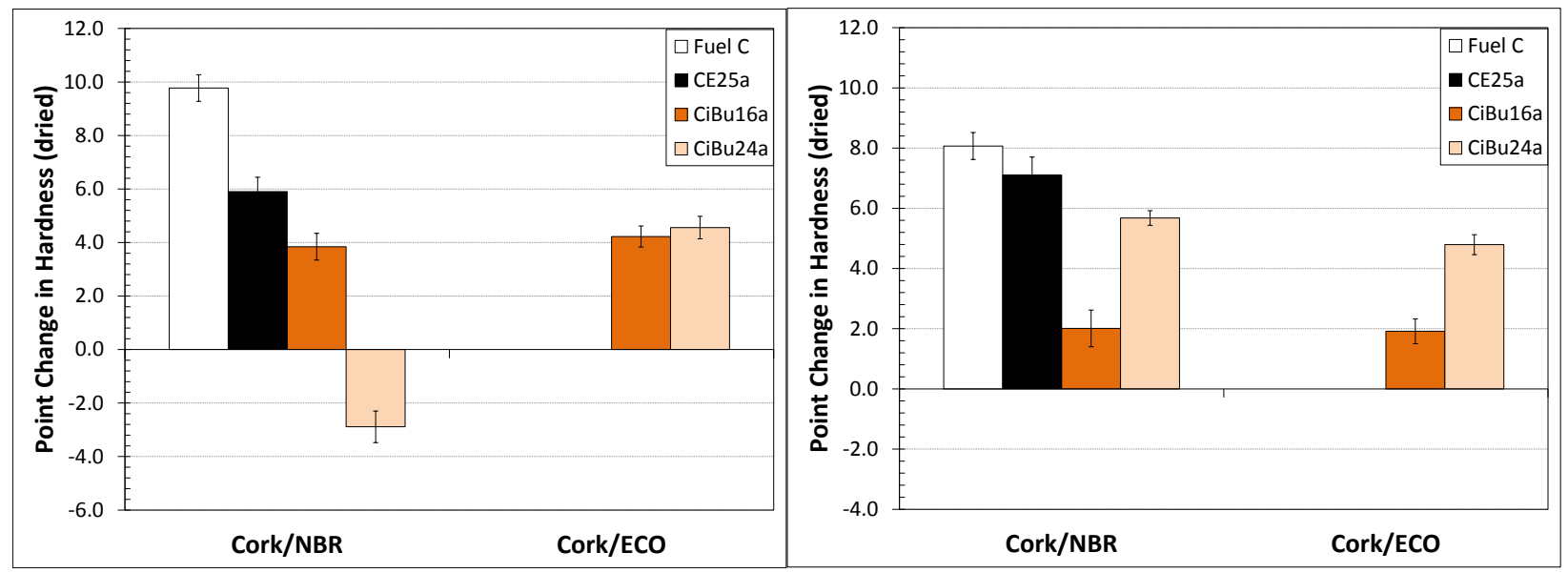

Fig. 66. Point change in hardness (after drying) for the cork materials. .

\subsubsection{Dynamic Mechanical Analysis Results for Plastics}

Dynamic mechanical analysis was performed on the plastic and rubberized-impregnated cork specimens exposed to the test fuel liquids and their vapor phases in order to measure the storage modulus as a function of temperature. This measurement is useful for identifying the temperature associated with the onset of the transition from a stiff, glassy state to a more pliable rubber state. At ambient conditions most of the plastics used in fuel storage systems are in the rigid (glassy) state. In contrast, the elastomeric materials are designed to be in the pliable (rubbery) state to maintain flexibility and sealability under the ambient range of temperatures. It is important to note that DMA does not necessarily reflect on solubility. It is primarily representative of molecular structural changes, which may not be indicated in volume swell and hardness measurements. The DMA curves for the plastic and cork specimens are shown in Appendix B.

\subsubsection{Permeation barrier plastics}

A key motivation for performing DMA was to measure the glass transition temperature of PET. PET or Mylar is a low-cost permeation barrier material and it had exhibited a dramatic drop in $\mathrm{T}_{\mathrm{g}}$ with exposure to the CE25a test fuel. This effect was not expected based on the other property changes for this material, but it does indicate that some level of micro-structural change had occurred. The influence of fuel chemistry on $\mathrm{T}_{\mathrm{g}}$ for permeation barrier plastic materials is shown in Fig. 67 for liquid- and vapor-phase exposures. Except for CE25a, the $T_{g}$ values for the four plastic types were not significantly changed with exposure to the test fuels and isobutanol appears to have no detrimental effect. However, the specimen exposed CE25a did experience a $30^{\circ} \mathrm{C}$ drop in $\mathrm{T}_{\mathrm{g}}$ with exposure, and the implication is that PET exposed to ethanol will be in the rubbery state at high ambient temperatures instead of in the desired rigid condition. This result is a strong indication that chemical changes had occurred in the polymer. The results for the specimens exposed to the vapor phase roughly parallel the results obtained for the liquid exposures. Unfortunately there were no vapor specimens of PET to provide comparison with the liquid results. 


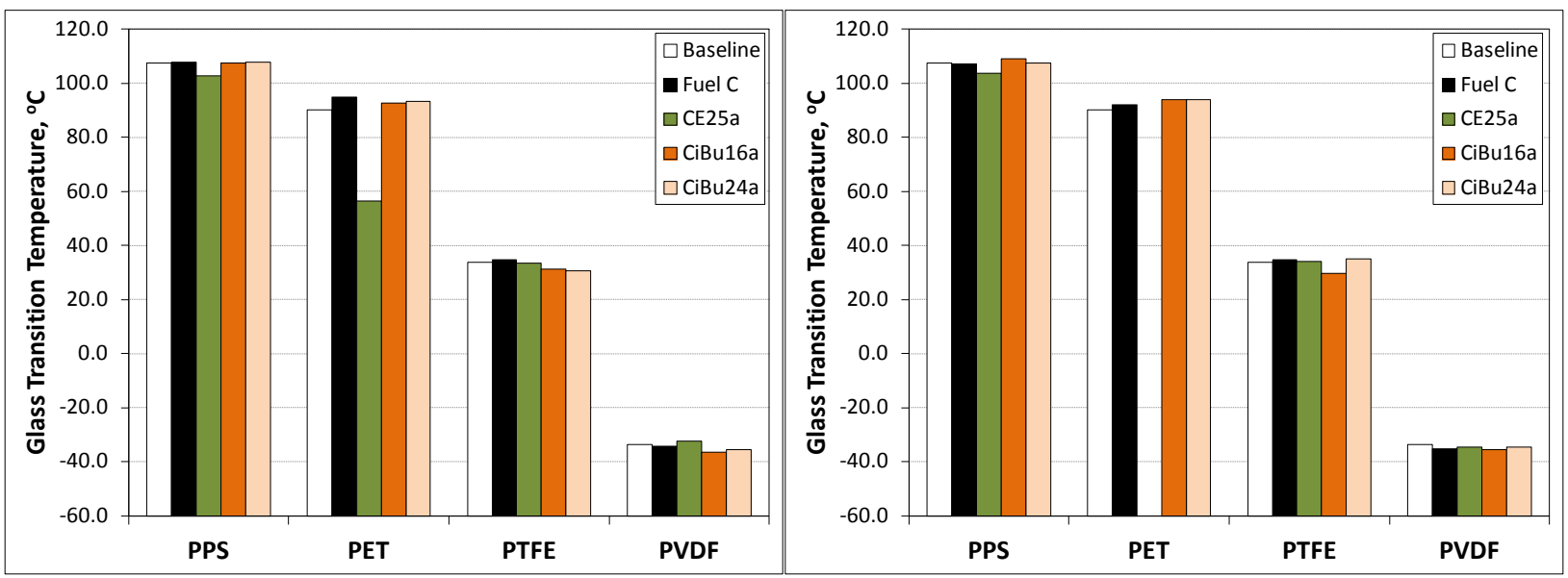

Fig. 67. Glass transition temperature results for permeation barrier plastics.

\subsubsection{Nylons and HDPE}

The glass transition temperature results for the four nylon materials and HDPE are shown in Fig. 68 and the results for the vapor exposures were similar to liquid exposure results. As shown in the figure, the $T_{g}$ value for HDPE was relatively unaffected following exposure to the test fuels. However, the results for the nylons varied considerably with both fuel chemistry and type of nylon. The $\mathrm{T}_{\mathrm{g}}$ value for Nylon 12 was raised around $20^{\circ} \mathrm{C}$ with exposure to Fuel C, while the addition of $25 \%$ aggressive ethanol had no appreciable effect. The test fuels containing isobutanol, on the other hand, did not appreciably affect $T_{g}$ from the original baseline result. Both Nylon 6 and Nylon 6,6 exhibited similar shifts in $T_{\mathrm{g}}$ with exposure to the test fuels. For these two nylons, Fuel $\mathrm{C}$ produced a small to moderate increase in $\mathrm{T}_{\mathrm{g}}$, while the test fuels containing isobutanol had little additional effect. However, exposure to CE25a caused $\mathrm{T}_{\mathrm{g}}$ to decline by $40^{\circ} \mathrm{C}$ and $60^{\circ} \mathrm{C}$ for Nylon 6 and Nylon 6,6, respectively. In contrast to the petroleum-derived nylons, Nylon 11 was unique in that the $\mathrm{T}_{\mathrm{g}}$ value decreased, rather than increased with exposure to Fuel C; this drop in $\mathrm{T}_{\mathrm{g}}$ was around $20^{\circ} \mathrm{C}$ from the original baseline value. CE25a caused $\mathrm{T}_{\mathrm{g}}$ of Nylon 11 to decline an additional $15^{\circ} \mathrm{C}$ and exposure to the isobutanol fuel blends resulted in $\mathrm{T}_{\mathrm{g}}$ decreasing approximately $60^{\circ} \mathrm{C}$, from the baseline value. Nylon 11 was the plastic most affected by aggressive isobutanol.

Liquid Exposures

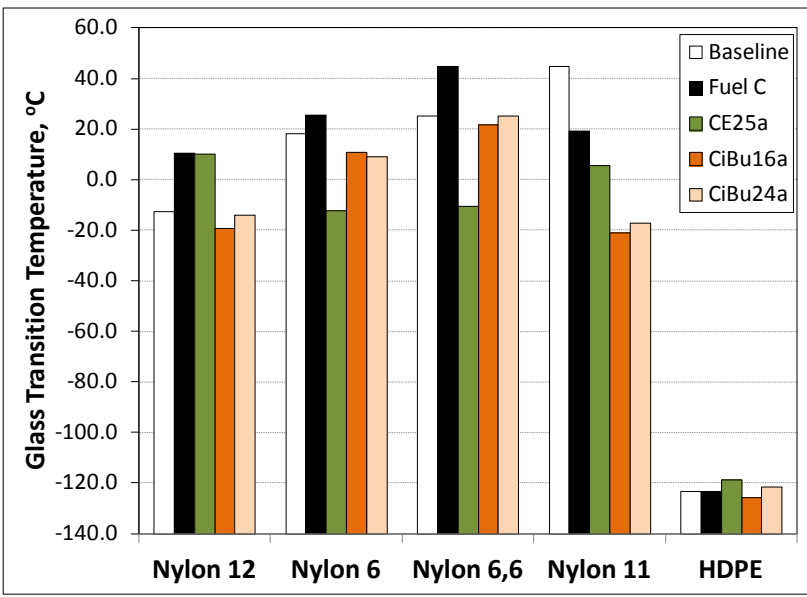

VaporExposures

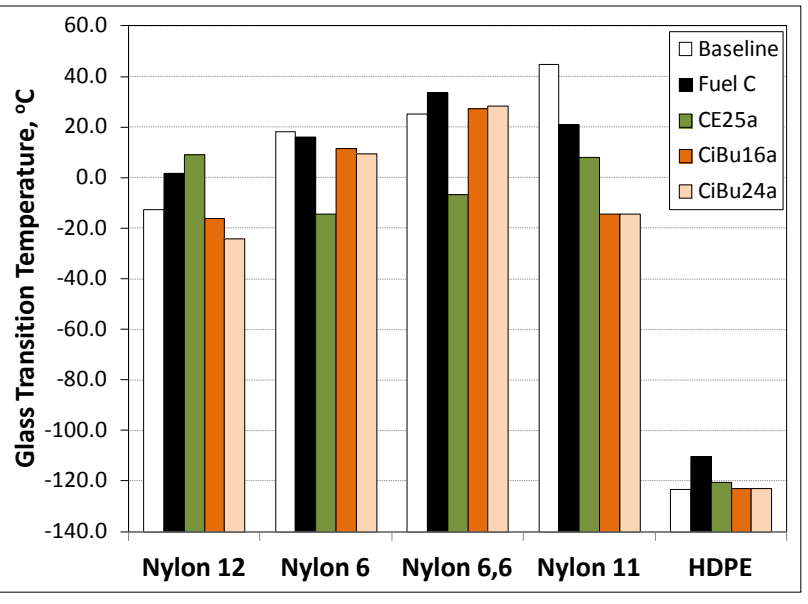

Fig. 68. Glass transition temperature results for the nylons and HDPE. 
The shift in $T_{g}$ observed for the nylon materials is especially important since the resulting shifts in values are within the range of outdoor ambient temperatures. For example, Nylon 6 and Nylon 6,6 are both designed to be in a rigid state for temperatures below $20^{\circ} \mathrm{C}\left(68^{\circ} \mathrm{F}\right)$ and in a pliable rubbery condition at higher temperatures. Exposure to CE25a reduced their $\mathrm{T}_{\mathrm{g}}$ values to between -10 and $-12^{\circ} \mathrm{C}\left(14\right.$ and $\left.10^{\circ} \mathrm{F}\right)$. As a result, these nylon types will be pliable for ambient temperatures well below their original baseline values. For the petroleum-derived nylons the addition of isobutanol to Fuel $\mathrm{C}$ appears to have only a small impact on the original $\mathrm{T}_{\mathrm{g}}$ value. In contrast to observed changes in volume swell and hardness that occurred with test fuel exposure, the shift in $\mathrm{T}_{\mathrm{g}}$ for the nylons caused by the alcohol additions did not correlate with their respective solubility curves The strong shifts in $\mathrm{T}_{\mathrm{g}}$ indicate that physical changes occurred to the original molecular structure have taken place to affect the point at which molecular relaxation occurs.

\subsubsection{Fiberglass resins}

The results for the two intact resin materials in Fig. 69 show that these two resins behave somewhat differently when exposed to the test fuels. Terephthalic polyester undergoes a $40^{\circ} \mathrm{C}$ drop in $\mathrm{T}_{\mathrm{g}}$ with exposure to Fuel $\mathrm{C}$, while $\mathrm{T}_{\mathrm{g}}$ for vinyl ester is unaffected. The addition of $25 \%$ aggressive ethanol to Fuel $\mathrm{C}$ significantly lowered the value of $\mathrm{T}_{\mathrm{g}}$ for the vinyl ester resin, but had only small effect (beyond Fuel C) for the terephthalic polyester sample. The test fuels containing isobutanol significantly lowered Tg for both materials, but for the vinyl ester the effect was less pronounced than for CE25a. The terephthalic polyester, on the other hand, exhibited the greatest decline in $\mathrm{T}_{\mathrm{g}}$ with the isobutanol additions.

Unfortunately, no CE25a exposures were performed in the vapor-phase region for comparison.

Liquid Exposures

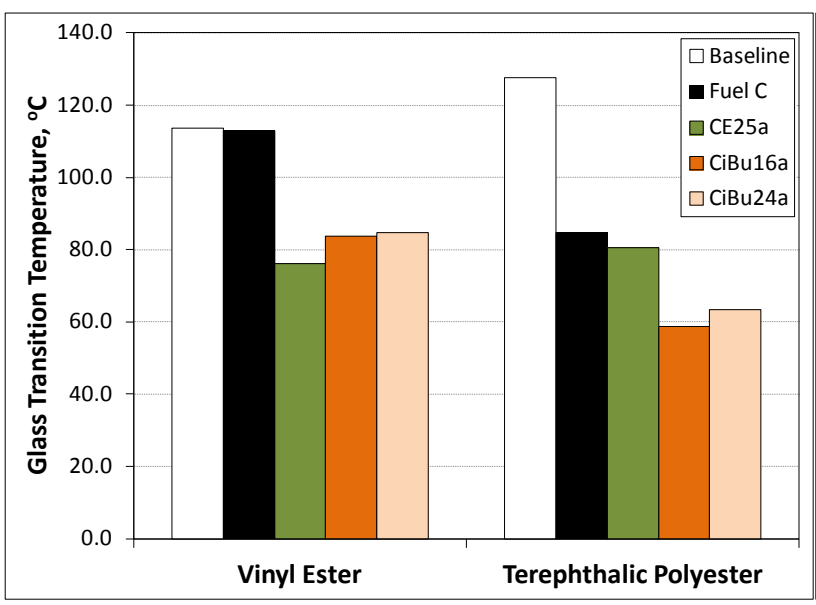

VaporExposures

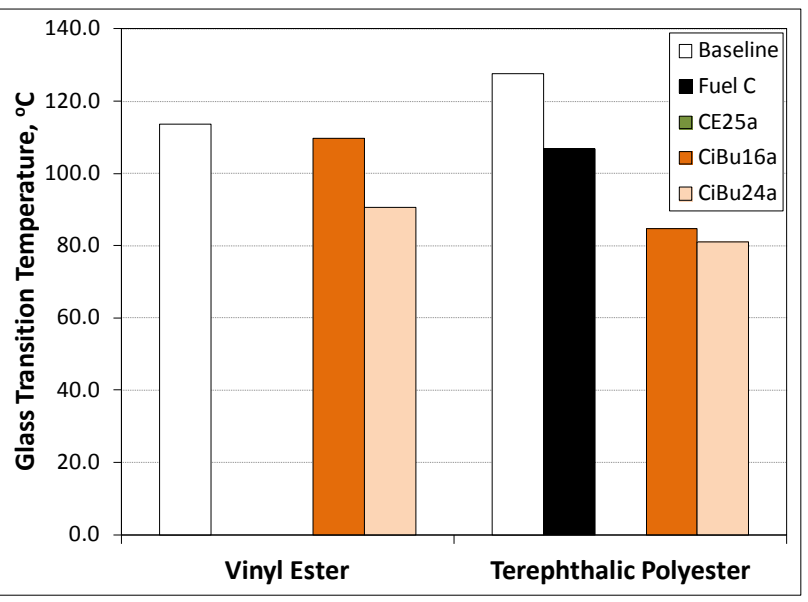

Fig. 69. Glass transition temperature results for two resin materials.

\subsubsection{Other common plastics}

The glass transition temperature results for the mid-range and commodity plastic materials are shown in Fig. 70. The two acetal plastic specimens, $\mathrm{POM}$ and the POM copolymer, did not undergo a shift in $\mathrm{T}_{\mathrm{g}}$ following exposure to the test fuels. However, the specimens composed of PBT, PP, and PETG all exhibited significant declines in $\mathrm{T}_{\mathrm{g}}$ accompanying exposure to Fuel C. PBT exhibited an additional $20^{\circ} \mathrm{C}$ decline in $\mathrm{T}_{\mathrm{g}}$ when exposed to CE25a, and an additional $15^{\circ} \mathrm{C}$ reduction for the test fuels containing isobutanol. 
Liquid Exposures

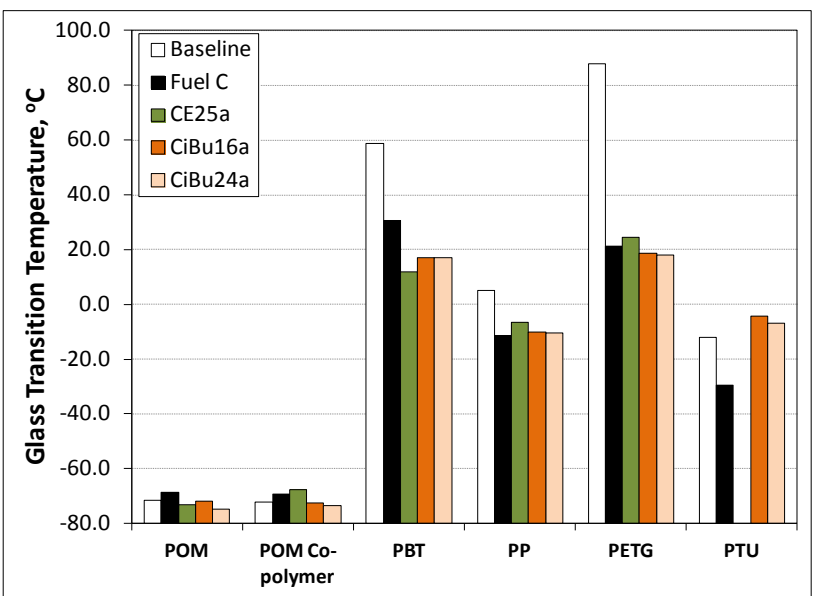

VaporExposures

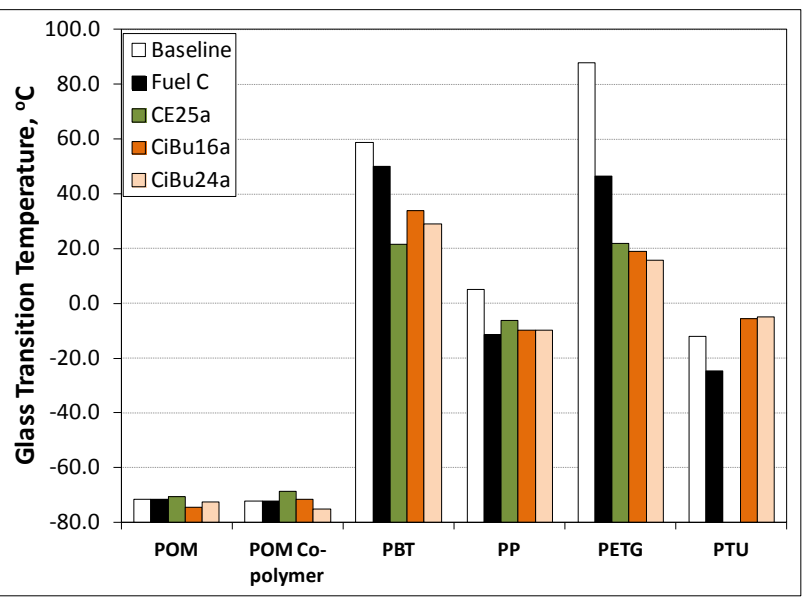

Fig. 70. Glass transition temperature results for other common plastic materials.

PP and PETG did not show any added effect when either alcohol type was added to the Fuel C formulation, indicating that Fuel $\mathrm{C}$ was primarily responsible for the observed downward shift. PTU exhibited a small decline in $\mathrm{T}_{\mathrm{g}}$ with Fuel $\mathrm{C}$ exposure, but the isobutanol was observed to produce a small increase in Tg (from baseline). PTU was not exposed to CE25a, so no data are available.

\subsubsection{Rubber-impregnated cork}

Two rubberized cork specimens were also evaluated and their DMA results are shown in Fig. 71 . The cork/ECO combination exhibited a small increase in $\mathrm{T}_{\mathrm{g}}$ with exposure to CiBu16a and CiBu24a over the baseline value. (There was no specimen evaluated in CE25a for comparison.) $\mathrm{T}_{\mathrm{g}}$ for the cork containing NBR was raised following exposure to Fuel C, but additions of aggressive isobutanol to the fuel mixture did not change $T_{\mathrm{g}}$ from the original baseline value. In general the specimens exposed to the vapors performed similarly to those submerged in the liquids.

\section{Liquid Exposures}

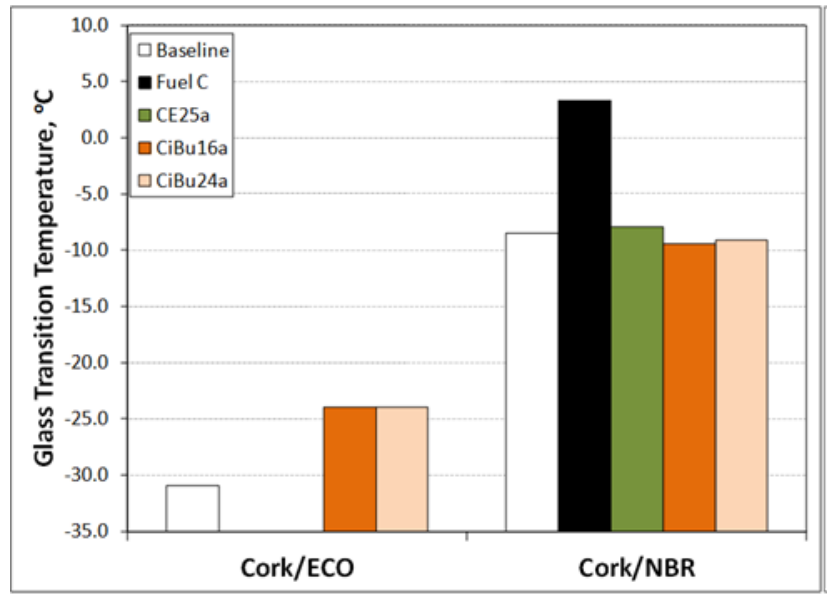

\section{Vapor Exposures}

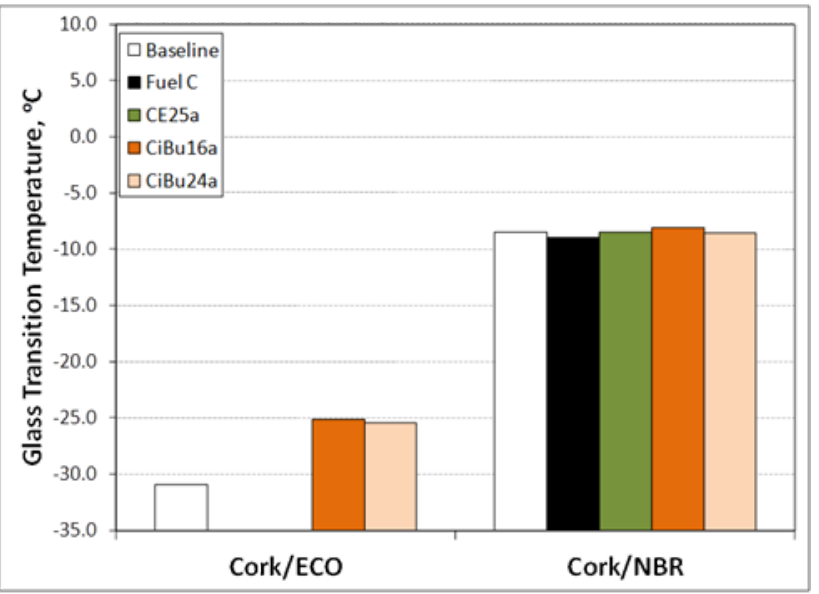

Fig. 71. Glass transition temperature results for the rubberized cork materials. 


\subsection{METALS}

In general the metals results obtained for the test fuels containing isobutanol (CiBu16a and CiBu24a) were similar to those obtained for test fuels containing ethanol. ${ }^{5}$ Liquid immersion exposures of brass, bronze, and zinc-plated (galvanized) steel produced pronounced discoloration and minor corrosion product film formation. None of the coupons suffered measureable thickness change and the original machining marks or plating grain structure remained visible on the coupon surface following exposure. Coupons exposed to the vapor phase above each solution exhibited slight discoloration (particularly the brass and bronze specimens) but no measureable mass loss was detected in any of these specimens. The results for the plated and galvanically coupled coupons also showed no significant corrosion. These specimens included steel coupons plated with chromium, nickel, lead, and zinc, chromium-plated brass and nickel-plated aluminum. Further, none of the plated coupons with substrates partially exposed revealed accelerated corrosion due to galvanic coupling between the plating material and substrate.

\subsubsection{Steel, Aluminum, and Nickel}

Metal coupons composed of 1020 mild steel, 1100 aluminum, 201 nickel, and 304 stainless steel were found to be essentially immune to corrosion by the test fuels for the exposure conditions provided by the environmental chambers. Similar observations were found for these metals when exposed to test fuels containing aggressive ethanol. ${ }^{3-5}$ There was essentially no weight loss, no discoloration, and no tendency toward localized corrosion under/near the crevice washers. The implication is that these materials are suitable for extended service in intermediate fuel blends of this type. Furthermore, when compared to metal corrosion in fuel mixtures containing ethanol, the coupons exposed to test fuels containing aggressive isobutanol exhibited similar and in some cases superior corrosion resistance. This effect is likely attributed to the fact that ethanol is much more hygroscopic than isobutanol.

\subsubsection{Brass, Bronze and Galvanized Steel}

Similar to earlier results in ethanol containing fuels, ${ }^{5}$ immersed coupons of cartridge brass, phosphor bronze, and zinc-plated (galvanized) steel exhibited variable degrees of discoloration and minor corrosion product film formation in the isobutanol blends. The highest corrosion rate observed among these very slightly susceptible materials was about $1 \mu \mathrm{m} / \mathrm{y}$ (calculated from an apparently uniform weight loss) for the cartridge brass and phosphor bronze. In most cases, the absolute value of the corrosion rate based on weight loss calculations is slightly compromised by minor amounts of adherent corrosion product - this tends to introduce a small under-estimation error in the corrosion rate calculation - but it should be noted that none of the coupons suffered measureable thickness change and the original machining marks or plating grain structure remained visible on the coupon surface following testing. Further, based on the initial testing, it is suspected that the corrosion rate of the copper-based materials is decreasing with extended exposure time. ${ }^{4}$

The photographs shown in Fig. 72 represent the unexposed and post-exposure appearance of coupons of cartridge brass, phosphor bronze, and galvanized steel. These images indicate the degree of discoloration and film formation associated with each exposure (other materials indicated far less, or even no discoloration and film formation). The degree of discoloration of these samples varied slightly between the CiBu16a and CiBu24a exposures, but there was no measureable difference of the corrosion rate based on the mass change as a function of isobutanol fraction in the fuel. Exposed specimens shown are opposite sides of the duplicate coupons. One side shows the imprint of the crevice washer slots and the other side shows the imprint of the shoulder washer. There was no evidence of pronounced crevice corrosion in any of the specimens. 

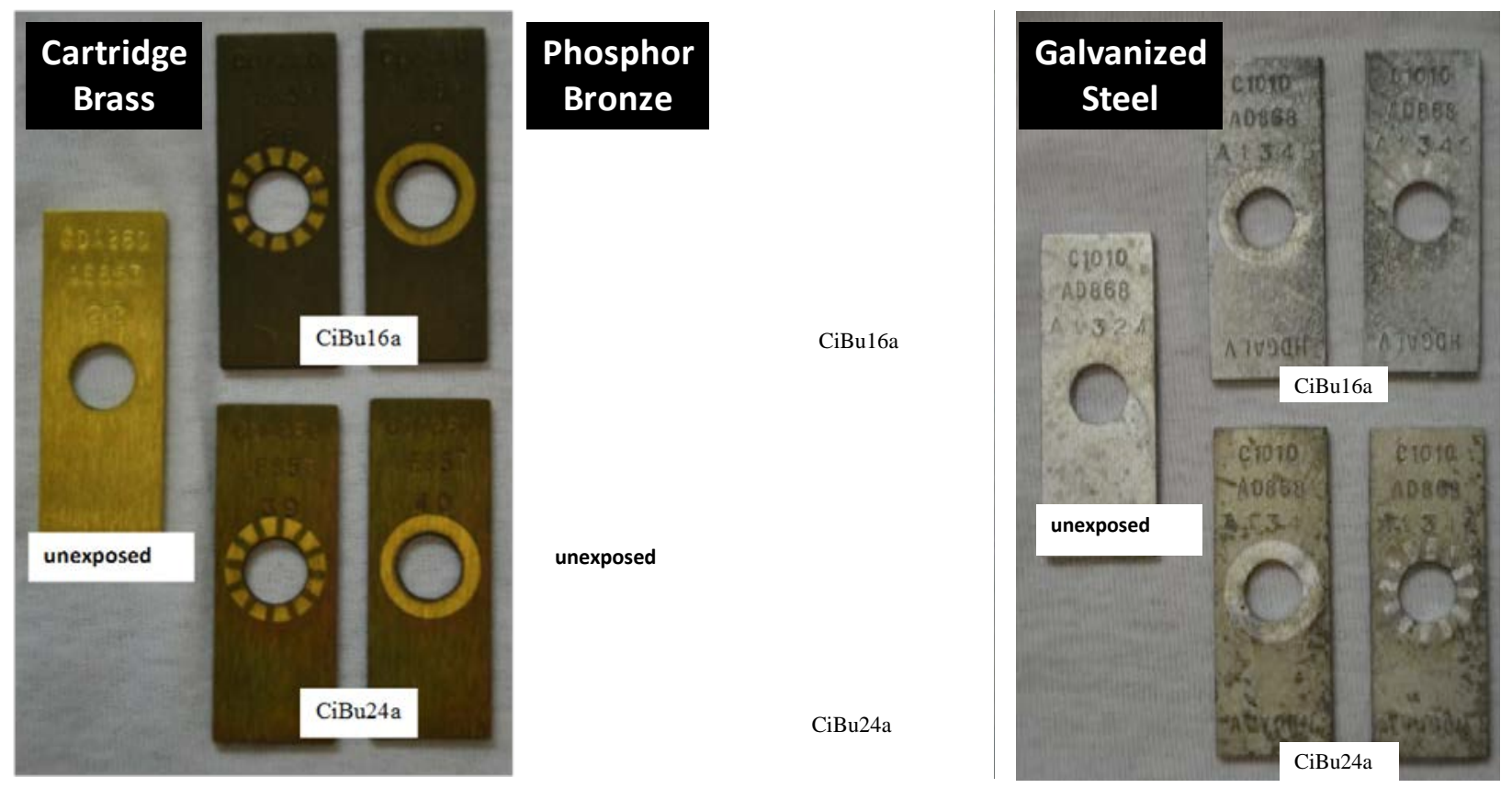

Fig. 72. Unexposed and post-exposure appearance of the cartridge brass, phosphor bronze, and galvanized steel specimens following 4 weeks of immersion in CiBu16a and $\mathrm{CiBu} 24 a$ at $60^{\circ} \mathrm{C}$.

Figures 73 and 74 show the post-exposure appearance of the other metal coupons including the plated and galvanic couple specimens exposed to CiBu16a. Photographs for the specimens exposed to CiBu24a look similar to the CiBu16a specimens and are not included. Mass changes for these samples were below the measurement threshold and there was no significant difference in the appearance of the exposed and unexposed specimens.

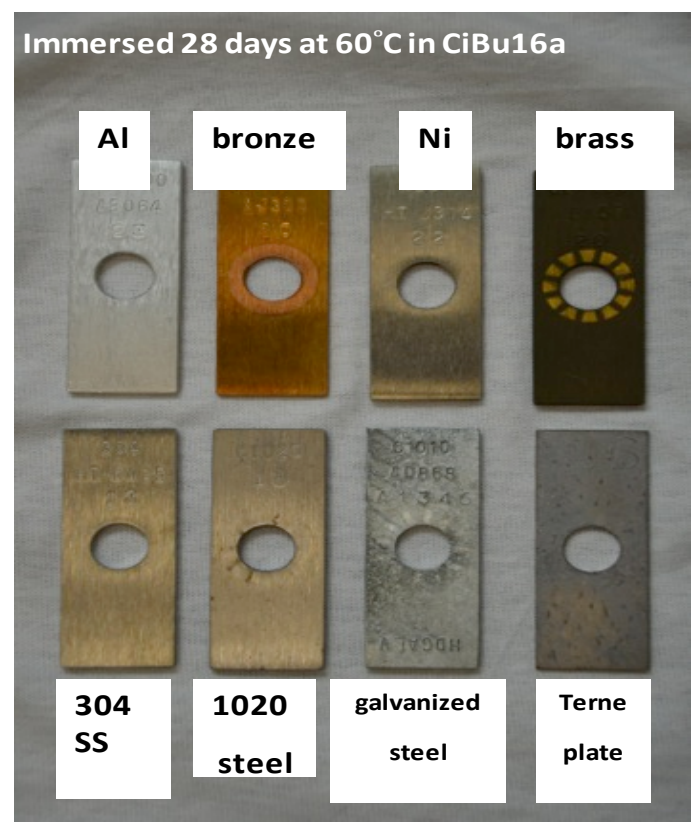

Fig. 73. Post-exposure appearance of other metallic specimens included in the study following 4 weeks of exposure to immersion in CiBu16a at $60^{\circ} \mathrm{C}$. 


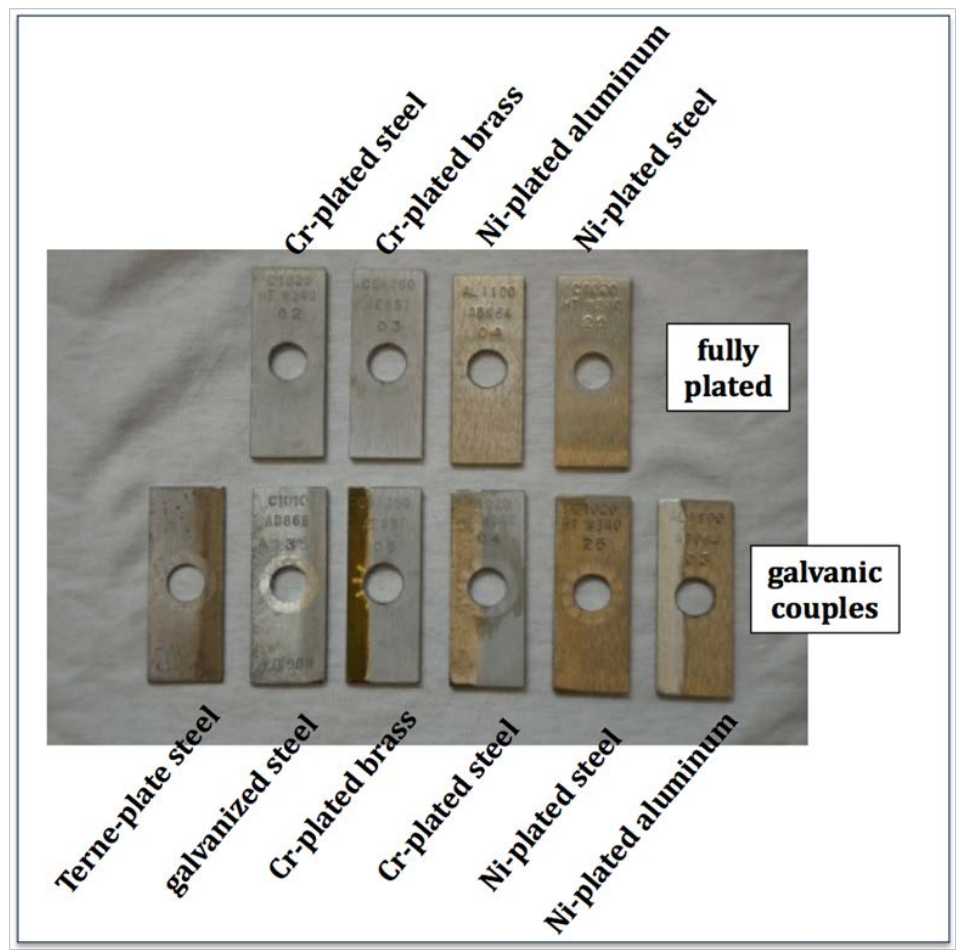

Fig. 74. Post-exposure appearance of other coated and galvanic coupled specimens included in the study following 4 weeks of exposure to immersion in $\mathrm{CiBu} 16 \mathrm{a}$ at $60^{\circ} \mathrm{C}$.

X-ray photoelectron spectroscopy (XPS) was performed on brass and bronze coupon surfaces following testing (to compare with results from unexposed coupons) to assess the corrosion film thickness and composition as a function of isobutanol content in the exposure environment. Four samples were examined, including phosphor bronze and cartridge brass exposed to both CiBu16a and CiBu24a. For each sample, two areas were analyzed; one area protected by the Teflon washer and one fully exposed to the fuel mixture. Survey scans were taken on the as-received surfaces and the results are shown in Table 7. For the phosphor bronze samples, the surfaces showed varying amounts of carbon, copper, zinc and oxygen as the predominant atomic species. Sulfur was detected in abundance on the exposed surface, but little was detected on the protected areas. Fluorine was detected in areas protected by the Teflon, the result of minute amounts of fluorine leaching from the fluoropolymer into the metal surface.

Table 7. XPS survey results on bronze and brass metal specimens

\begin{tabular}{lccccccccccc}
\hline \multirow{2}{*}{ Metal Type } & \multirow{2}{*}{ Test Fuel } & \multirow{2}{*}{$\begin{array}{c}\text { Surface } \\
\text { Condition }\end{array}$} & \multicolumn{8}{c}{ Elemental Surface Composition (at. \%) } \\
\cline { 5 - 12 } & & & $\mathbf{C}$ & $\mathbf{C u}$ & Zn & $\mathbf{O}$ & $\mathbf{S}$ & N & Na & Ca & F \\
\hline Phosphor bronze & CiBu16a & Clean & 79.9 & 1.1 & 0.2 & 14.5 & 0.8 & 1.7 & 0.1 & 1.4 & 0.4 \\
Phosphor bronze & CiBu16a & Exposed & 75.9 & 2.0 & 0.4 & 13.5 & 6.7 & 0.8 & 0.1 & 0.7 & 0.0 \\
Phosphor bronze & CiBu24a & Clean & 71.7 & 3.0 & 0.3 & 17.0 & 0.7 & 5.6 & 0.1 & 0.4 & 1.3 \\
Phosphor bronze & CiBu24a & Exposed & 67.5 & 5.6 & 0.1 & 11.7 & 12.5 & 2.3 & 0.1 & 0.0 & 0.0 \\
Cartridge brass & CiBu16a & Clean & 68.6 & 3.2 & 0.7 & 18.6 & 1.3 & 5.3 & 0.3 & 0.8 & 1.1 \\
Cartridge brass & CiBu16a & Exposed & 63.3 & 2.6 & 1.3 & 22.1 & 7.3 & 1.5 & 1.2 & 0.4 & 0.3 \\
Cartridge brass & CiBu24a & Clean & 70.8 & 3.0 & 0.4 & 12.5 & 0.3 & 7.5 & 0.2 & 5.0 & 0.4 \\
Cartridge brass & CiBu24a & Exposed & 68.8 & 5.0 & 0.4 & 12.0 & 11.0 & 2.3 & 0.1 & 0.5 & 0.0 \\
\hline
\end{tabular}


Minor amounts of N, Na, and Ca were also found to vary slightly across the clean versus the exposed surfaces. These minor elements are reported for the sake of completeness, but they do not represent any trend above the scatter which would be expected in an experiment of this nature. Similar trends were observed for the cartridge brass samples. The key result from the surface assessment is that the exposed surfaces in both phosphor bronze and cartridge brass have substantial changes in sulfur content on the surface which displaces other film producing agents such as oxygen and nitrogen. The sulfide layer is thin and protective and not a cause for concern from a corrosion standpoint.

Representative XPS depth profiles for the clean and exposed surfaces for phosphor bronze exposed to CiBu16a are shown in Figs. 75 and 76. (Note that the units on the y-axis represent atom percentages.) They reveal the composition of the corrosion product film as a function of depth from the surface (the film is incrementally sputtered from the surface and the identity of released materials is distinguished by relative energy). The depth profiles for phosphor bronze are not shown for samples exposed to CiBu24a because they yielded essentially identical results as the CiBu16a exposures. The protected area of the phosphor bronze in both the CiBu16a and CiBu24a exposures showed a rapid loss of carbon and oxygen as sputtering proceeded deeper into the sample and an increase of copper and tin. The profile is generally complete (meaning it is unchanging) by $\sim 50 \mathrm{~nm}$, at which point the oxygen has dropped to less than half of its initial value. The profile shows a thin native oxide which is primarily tin oxide.

The exposed areas for the phosphor bronze in CiBu16a and CiBu24a show a complex set of layers on the surface caused by exposure to the fuel mixtures. To sort out the profiles, the tin and copper signals were deconvoluted to separate them into the metal and metal-oxide components. The outermost layer consists of a copper sulfide that incorporates carbon and nitrogen. Just below the copper sulfide layer is a zinc oxide layer. Closest to the base metal is a thin tin oxide layer. Finally, the metal signals of tin and copper emerge underneath the sulfide and oxide layers. The total thickness of the sulfide and oxide layers is approximately $100 \mathrm{~nm}$.

The depth profiles for cartridge brass exposed to CiBu16a are shown in Figs. 77 and 78. Again, the profiles for cartridge brass exposed to $\mathrm{CiBu} 24 \mathrm{a}$ are not shown because they yielded essentially identical results to the CiBu16a exposures. The protected area of the cartridge brass in both the CiBu16a and CiBU24a exposures showed a rapid loss of carbon and oxygen as sputtering proceeded deeper into the sample, an increase of copper, a layer of zinc oxide and then an increase of zinc metal base material. The zinc signal was deconvoluted into metal and metal oxide portions. The profiles are generally complete by approximately $100 \mathrm{~nm}$. There is a thin layer of primarily zinc oxide on the surface.

Similar to phosphor bronze, the exposed areas for the cartridge brass CiBu16a and CiBu24a show a complex set of layers on the surface caused by exposure to the fuel mixtures. The outermost layer consists of a copper sulfide and copper oxide that incorporates carbon and nitrogen, with zinc relatively absent. Below this layer is a substantial zinc oxide layer which extends to a depth approaching $400 \mathrm{~nm}$, with copper relatively absent between 100-300 nm. Metallic zinc begins to emerge at a depth of approximately $200 \mathrm{~nm}$. Of all the materials tested, cartridge brass exhibited the highest corrosion rate of only $\sim 1 \mu \mathrm{m} /$ year, and the XPS results are consistent with very limited surface changes and product films $\leq 400 \mathrm{~nm}$ in thickness. 


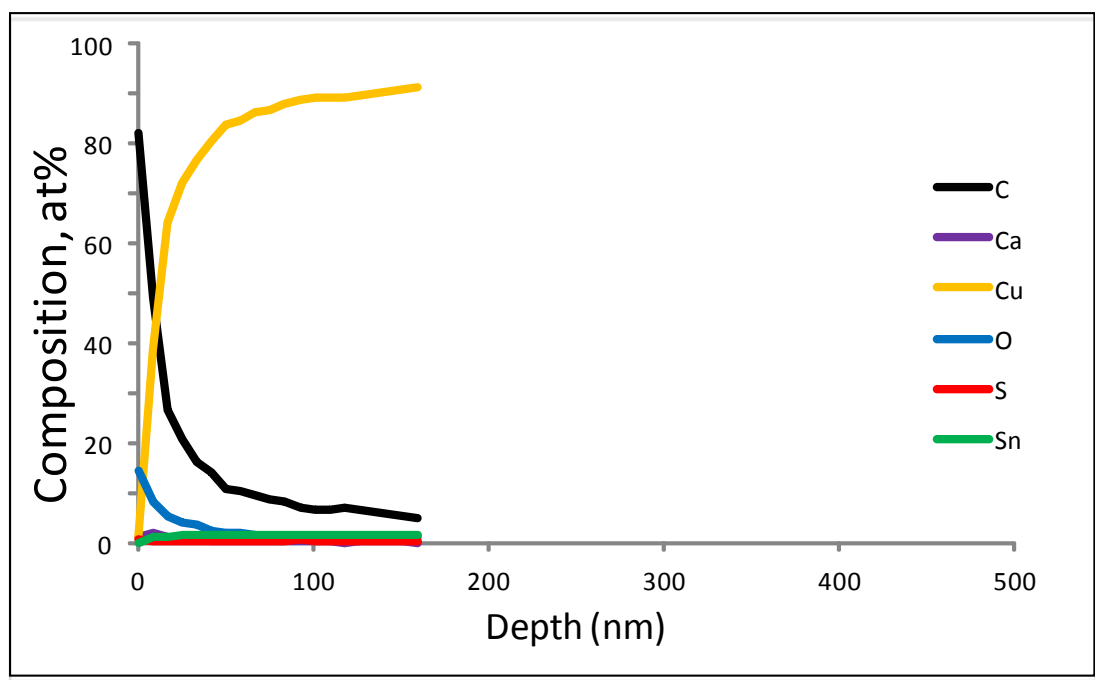

Fig. 75. XPS depth profile for phosphor bronze on an area of the surface protected by a Teflon washer following immersion in CiBu16a for 4 weeks at $60^{\circ} \mathrm{C}$.

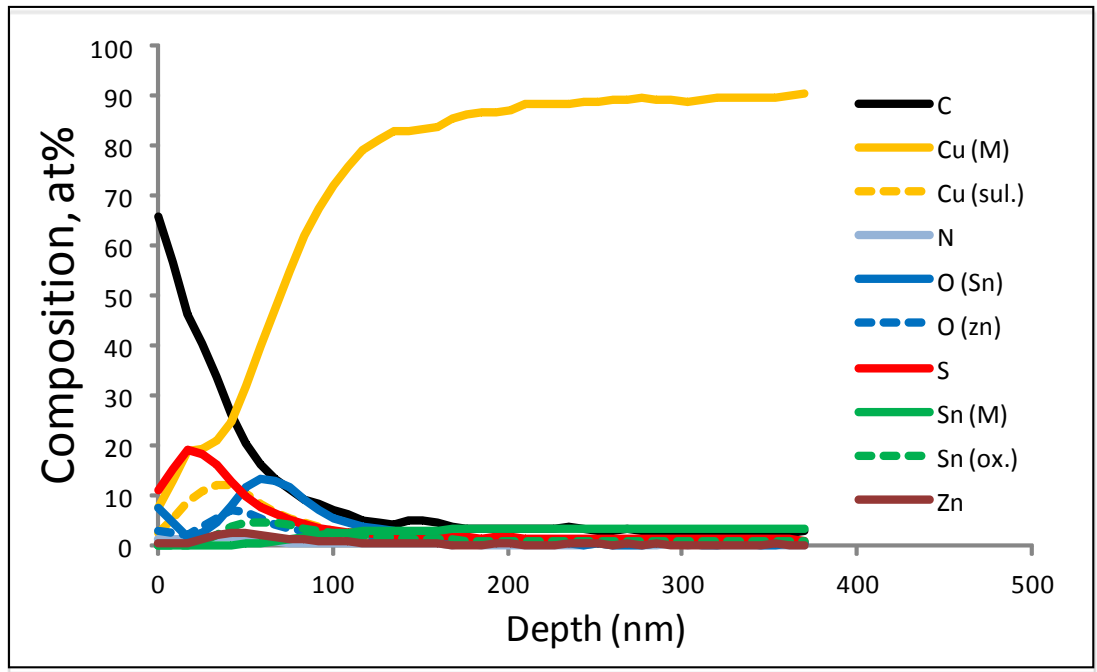

Fig. 76. XPS depth profile for phosphor bronze on an exposed area of the surface following immersion in CiBu16a for 4 weeks at $60^{\circ} \mathrm{C}$. 


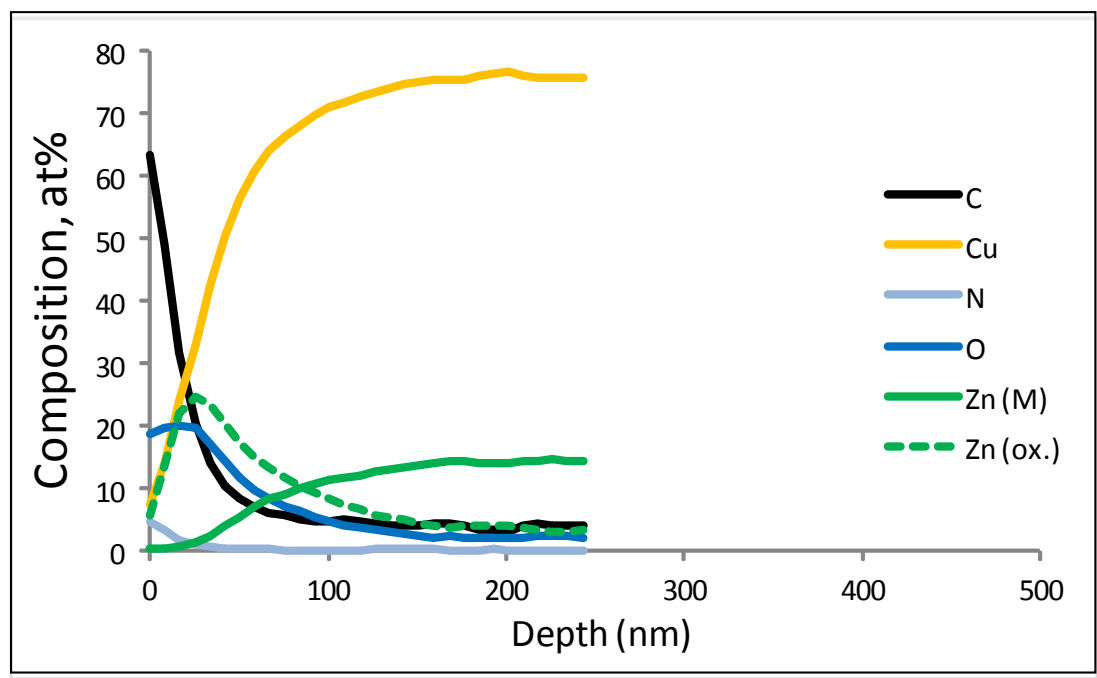

Fig. 77. XPS depth profile for cartridge brass on an area of the surface protected by a Teflon washer following immersion in CiBu16a for 4 weeks at $60^{\circ} \mathrm{C}$.

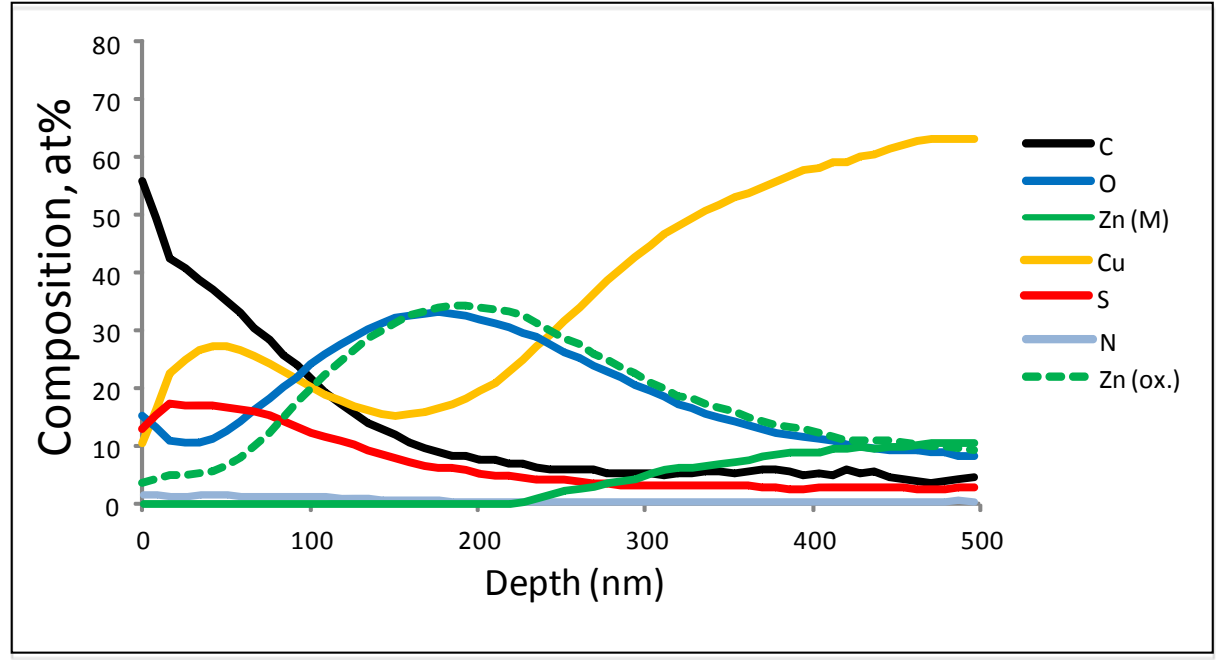

Fig. 78. XPS depth profile for cartridge brass on an exposed area of the surface following immersion in CiBu16a for 4 weeks at $60^{\circ} \mathrm{C}$. 


\section{CONCLUSIONS}

In this study two isobutanol test fuels ( $\mathrm{CiBu} 16 \mathrm{a}$ and $\mathrm{CiBu} 24 \mathrm{a}$ ) were prepared to assess the compatibility of infrastructure materials (elastomers, plastics, and metals) with gasoline containing isobutanol. An aggressive isobutanol fuel formulation was developed based on the aggressive alcohol formulations described in SAE J1681. The results from these test fuels were compared to oxygen equivalent aggressive ethanol formulations (CE10a and CE17a) for the elastomeric materials and to CE25a for the plastics. A solubility analysis was also performed using Hansen solubility parameters to assess the potential solubility of the polymer materials to gasoline containing isobutanol or ethanol. In most cases the solubility potential was slightly lower for isobutanol blends compared to the ethanol blends, and experimental results were largely in agreement with the isobutanol blend results being similar to, or better than the ethanol blend results.

\subsection{ELASTOMERS}

Seven elastomeric types were evaluated for their compatibility to gasoline containing 16 and 24\% isobutanol. These materials include representatives of fluorocarbon, fluorosilicone, NBR, neoprene, SBR, polyurethane, and silicone rubbers. In general the observed mass and volume increase was found to correlate with the predicted solubility. A ranking of the elastomer types from the lowest to the highest degree of swelling is as follows:

- Fluorocarbon

- Fluorosilicone

- NBR

- Polyurethane

- Neoprene

- SBR

- Silicone

The observed swell for the elastomers for exposures to CiBu16a and CiBu24a were similar to, but slightly less than the oxygen equivalent ethanol fuels of CE10a and CE17a. The softening which accompanied volume expansion was significant and roughly proportional to the degree of swell, except for polyurethane which was dramatically softened preferentially by the ethanol-gasoline test fuels. More softening occurred with CE10a and CE17a, than CiBu16a and CiBu24a. When dried, the properties for fluoroelastomers and silicone rubbers returned to values close to the original condition. The NBRs, neoprene and SBR exhibited significant shrinkage with exposure to the tests fuels and the observed shrinkage was roughly the same for each fuel type. For most of the NBRs, higher embrittlement was observed with exposure to Fuel C and CE10a; than with the isobutanol test fuels. These results suggest that Fuel C was primarily responsible for the dissolution and extraction in NBR, neoprene and SBR. The performance of the specimens in the vapor phase roughly corresponded to the property changes of the liquid exposures, with one exception. Five of the six NBRs showed pronounced embrittlement with CE10a and C17a vapors, indicating that the presence of ethanol in the vapor phase will produce significant embrittlement in many NBR types.

In marked contrast to the other elastomers, polyurethane was unaffected by Fuel C, but exposure to either ethanol or isobutanol degraded it performance. A small level of shrinkage was observed following exposure to CE17a, CiBu16a, and CiBu24a, but CE10a produced significantly higher shrinkage in this material. Polyurethane was also unique in that it was the only elastomer which lost mass and hardness after being dried following exposure to the alcohol test fuels. This result suggests that polyurethane was 
chemically degraded to some extent by the alcohols, although isobutanol was less effective than ethanol at altering its properties.

\subsection{PLASTICS (AND CORK)}

Of all the plastic materials evaluated in this study, the permeation barrier materials, PPS and PTFE, were not significantly affected by the test fuels. At first glance PET appeared to be unaffected as well since its mass, volume, and hardness were not noticeably changed. However, CE25a was observed to significantly lower the glass-to-rubber transition temperature, indicating that structural changes had occurred in the PET specimens with exposure to this fuel. PVDF, POM, POM copolymer, PBT and PP exhibited around $5 \%$ swelling and were notably softened, but they returned to their original mass, volume and hardness values after drying. Fuel $\mathrm{C}$ was observed to lower the glass-to-rubber transition temperatures for PP and especially PBT, but PVDF, POM, POM copolymer returned to their baseline temperatures. It is worth noting that PVDF exhibited pronounced swelling with exposure to CE25a compared to the other test fuels.

HDPE swelled around 10\% and was softened when exposed to the test fuels. Fuel C was primarily responsible as the alcohol additions had no additional effect. When dried, it returned to its baseline values.

The nylons were greatly affected by the test fuel chemistries. Nylon 6 and nylon 6,6 are similar in structure, and as expected, exhibited similar property changes. The volume and mass for these two nylons were unaffected for Fuel C, CiBu16a and CiBu24a, but CE25a produced significant swell and softening. This swell and hardness decrease remained even in the dried state, and it was noted that the glass-torubber transition temperature was lowered by $40^{\circ} \mathrm{C}$ or more by CE25a. Nylon 12 was not affected by Fuel $\mathrm{C}$ (when wetted), but significant swelling and softening occurred with exposure to CE25a, CiBu16a, and CiBu24a. When dried, Nylon 12 shrank around 5\% with Fuel C and the two isobutanol fuel blends. It shrank around 8\% with CE25a. The performance of Nylon 11 was notably different from the other nylons, which is not surprising since, unlike the others, it is made from vegetable oil. Nylon 11 was observed to swell 5\% with Fuel C exposure, 15\% with CiBu16a and CiBu24a, and 18\% for CE25a. This swell was accompanied by a high level of softening with CE25a, much less softening occurred with CiBu16a and CiBu24a. Nylon 11 remained swollen and softened even after drying following exposure to the test fuels containing ethanol and isobutanol. Nylon 11 was also unusual in that the glass-to-rubber transition temperature was lowered by exposure to Fuel $\mathrm{C}$ and its $\mathrm{T}_{\mathrm{g}}$ was further reduced by CE25a and even more so with CiBu16a and CiBu24a.

Polypropylene and PETG swelled the highest and softened the most of the plastics studied in this investigation. Fuel $\mathrm{C}$ was primarily responsible, although additional swelling (and softening) was observed for PETG when exposed to CE25a, CiBu16a, and CiBu24a. PP returned to its original condition when dried, but PETG remained in a highly swollen (and softened) state and its $\mathrm{T}_{\mathrm{g}}$ values were lowered as well.

PTU was the plastic most affected by the presence of ethanol or isobutanol. When wetted, it lost significant mass and volume with exposure to CE25a. The hardness for PTU was unaffected by Fuel C, but the alcohol additions produced the highest level of softening observed for any plastic material. When dried this material shrank when exposed to the fuels containing ethanol and isobutanol and was embrittled by each test fuel.

The epoxies and isophthalic resins had degraded substantially following exposure to the test fuels containing either isobutanol or ethanol. Only the vinyl ester and terephthalic polyester resins remained intact. Of the two surviving resins, the vinyl ester exhibited significantly better compatibility to the test 
fuels than the terephthalic polyester resin. These two resins exhibited some swelling with the Fuel C composition, but swelling (and softening) was more pronounced with CE25a, CiBu16a, and CiBu24a. CE25 produced more swell and softening than the isobutanol fuels. When dried, both resins remained swollen (and softened). For vinyl ester, the dried volume (and softening) was highest for CE25, while for terephthalic polyester, CiBu24a produced the highest dried volume. The glass-to-rubber transition temperatures for the vinyl ester was unaffected by Fuel C, but was lowered by the fuels containing the alcohols. In contrast, $\mathrm{T}_{\mathrm{g}}$ for the terephthalic polyester was lowered by Fuel $\mathrm{C}$, and even more so by the two test fuels containing isobutanol.

The cork specimens exhibited pronounced swelling and softening with exposure to the test fuels. Cork/NBR swelled from $10 \%$ for Fuel C to $40 \%$ or more when ethanol or isobutanol was present. Cork/ECO swelled significantly with the isobutanol fuels, but its performance to Fuel C and CE25a is unknown. The corks specimens shrank and were hardened following fuel exposure and, for cork/NBR the alcohols (and especially ethanol) were primarily responsible for the observed shrinkage (and hardening).

\subsection{METALS}

Metal specimens representing a variety of materials commonly found in fuel storage and dispensing systems were immersed in $\mathrm{CiBu} 16 \mathrm{a}$ and $\mathrm{CiBu} 24 \mathrm{a}$ fuel blends at $60^{\circ} \mathrm{C}$ and exposed to the vapors above these solutions for 28 days. In all cases, the annualized corrosion rates based on weight loss were negligible, less than $1 \mu \mathrm{m} / \mathrm{y}$ for cartridge brass and undetectable for the remaining metals in the study. Corrosion films were examined with XPS for the discolored specimens of cartridge brass and phosphor bronze, and the surface of each was found to contain copper sulfide. The thickness of each film was not found to be dependent on the concentration of isobutanol in the fuel blend. The results of this study confirm that 1100 aluminum, 1020 mild steel, 304 stainless steel, cartridge brass, phosphor bronze, 201 nickel, Cr-plated steel, Ni-plated steel, Cr-plated brass, Zn-plated steel, Ni-plated aluminum, and Pbplated steel would be compatible with isobutanol-containing fuels under the prescribed environmental conditions. 



\section{REFERENCES}

1. https://www.federalregister.gov/articles/2012/06/07/2012-13823/regulation-of-fuel-and-fueladditives-modification-to-octamix-waiver-txceed.

2. Partial Grant of Clean Air Act Waiver Application Submitted by Growth Energy to Increase Allowable Ethanol Content of Gasoline to 15 Percent; Decision of the Administrator (EPA-HQ-OAR2009-0211; FRL-9258-6), Federal Register 76(17), 4662 (Jan. 26, 2011); www.epa.gov/otaq/regs/fuel/additive/e15.\#wn.

3. M. D. Kass, T. J. Theiss, C. J. Janke, S. J. Pawel, and S. A. Lewis, Intermediate Ethanol Blends Infrastructure Materials Compatibility Study: Elastomers, Metals, and Sealants, report ORNL/TM-2010/326, Oak Ridge National Laboratory, March 2011.

4. M. D. Kass, T. J. Theiss, C. J. Janke, and S. J. Pawel, Compatibility Study for Plastic, Elastomeric, and Metallic Fueling Infrastructure Materials Exposed to Aggressive Formulations of EthanolBlended Gasoline, report ORNL/TM-2012/88, Oak Ridge National Laboratory, May 2012.

5. S. J. Pawel, M. D. Kass, and C. J. Janke, Preliminary Compatibility Assessment of Metallic Dispenser Materials for Service in Ethanol Fuel Blends, report ORNL/TM-2009/086, Oak Ridge National Laboratory, November 2009.

6. Society of Automotive Engineers, "Gasoline, Alcohol, and Diesel Fuel Surrogates for Materials Testing,” SAE J1681, issued September 1993, revised January 2000-01.

7. J. Baustian and L. Wolf, “Cold Start/Warm-up Vehicle Performance and Driveability Index for Gasolines Containing Isobutanol,” SAE Paper No. 2012-01-1741, SAE International, September 2012.

8. K. Kimura, L. Wolf, J. Baustian, and H Haskew, "Hydrocarbon Permeation in Gasoline Vehicle Fuel Systems Using Isobuanol Blends,” SAE Paper No. 2012-01-1582, SAE International, September 2012.

9. C. M. Hansen, Hansen Solubility Parameters: A User's Handbook, 2nd ed., CRC Press, Boca Raton, Florida, 2007.

10. K. M. Evans and J. K. Hardy, "Predicting Solubility and Permeation Properties of Organic Solvents in Viton Glove Material Using Hansen’s Solubility Parameters,” Journal of Applied Polymer Science 93(6), 2688-2698 (2004).

11. L. M. Robeson, Polymer Blends, A Comprehensive Review, Hanser Gardner Publications, Cincinnati, Ohio, 2007.

12. Parker O-Ring Handbook, ORD 5700, Parker Hannifin Corporation, Cleveland, Ohio, 2007.

13. Dichtomatik O-Ring Handbook, D. Visscher, Ed., Dichtomatik North America, 47690 East Anchor Court, Plymouth, Michigan.

14. R. Flitney, Seals and Sealing Handbook, 5th ed., Butterworth-Heinemann, Burlington, Massachusetts, 2007.

15. K. P. Menard, Dynamic Mechanical Analysis: A Practical Introduction, 2nd ed., CRC Press, Boca Raton, Florida.

16. NREL data sheet provided by Kristi Moriarty on January 13, 2011.

17. Butamax Advanced Biofuels Website, http://www.butamax.com. 

APPENDIX A. DMA RESULTS FOR ELASTOMERS 



\section{Viton A401C DMA Results for Control and Fuel C Exposures}
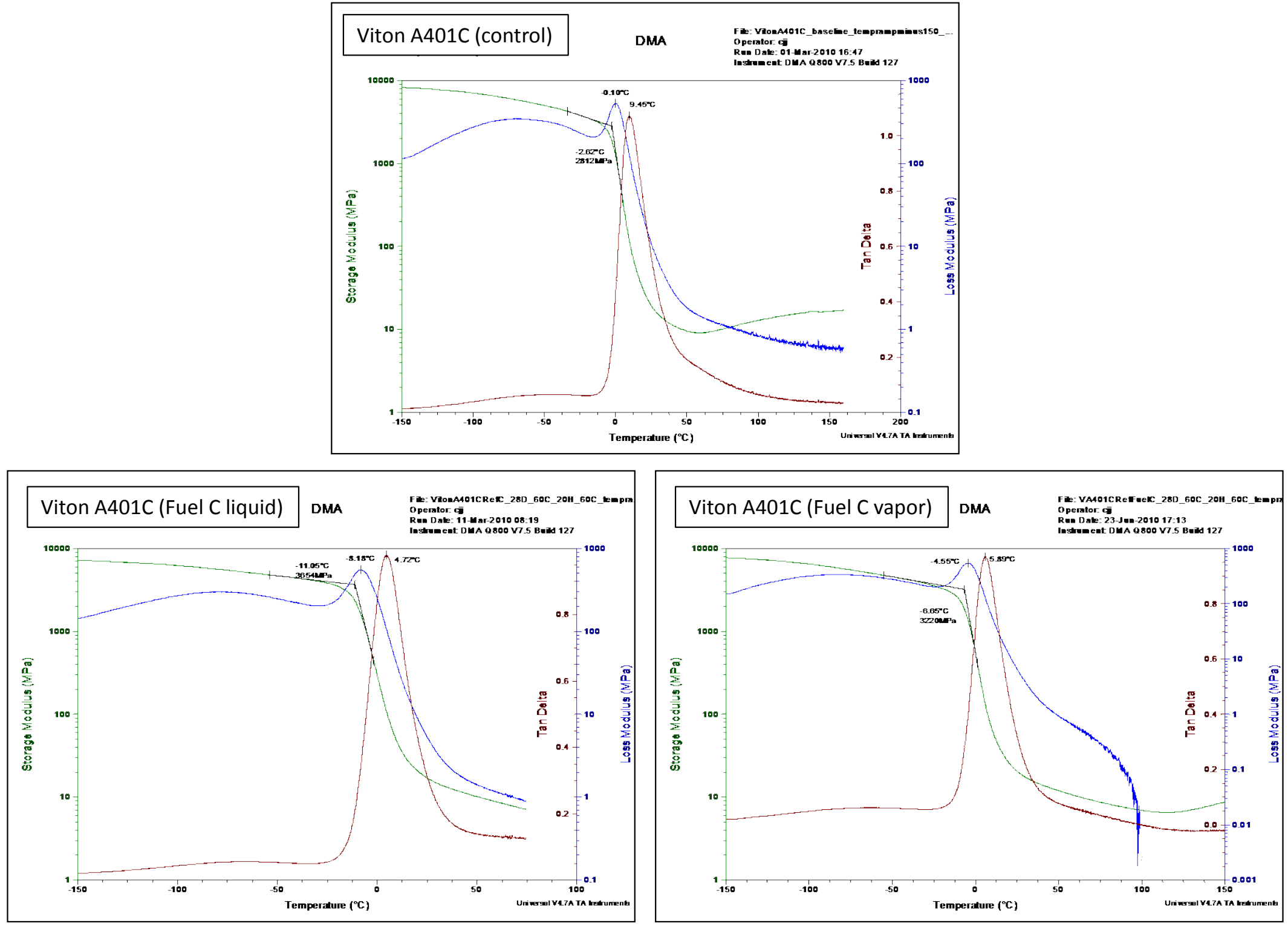
Viton A401C DMA Results for CE10a and C17a Exposures
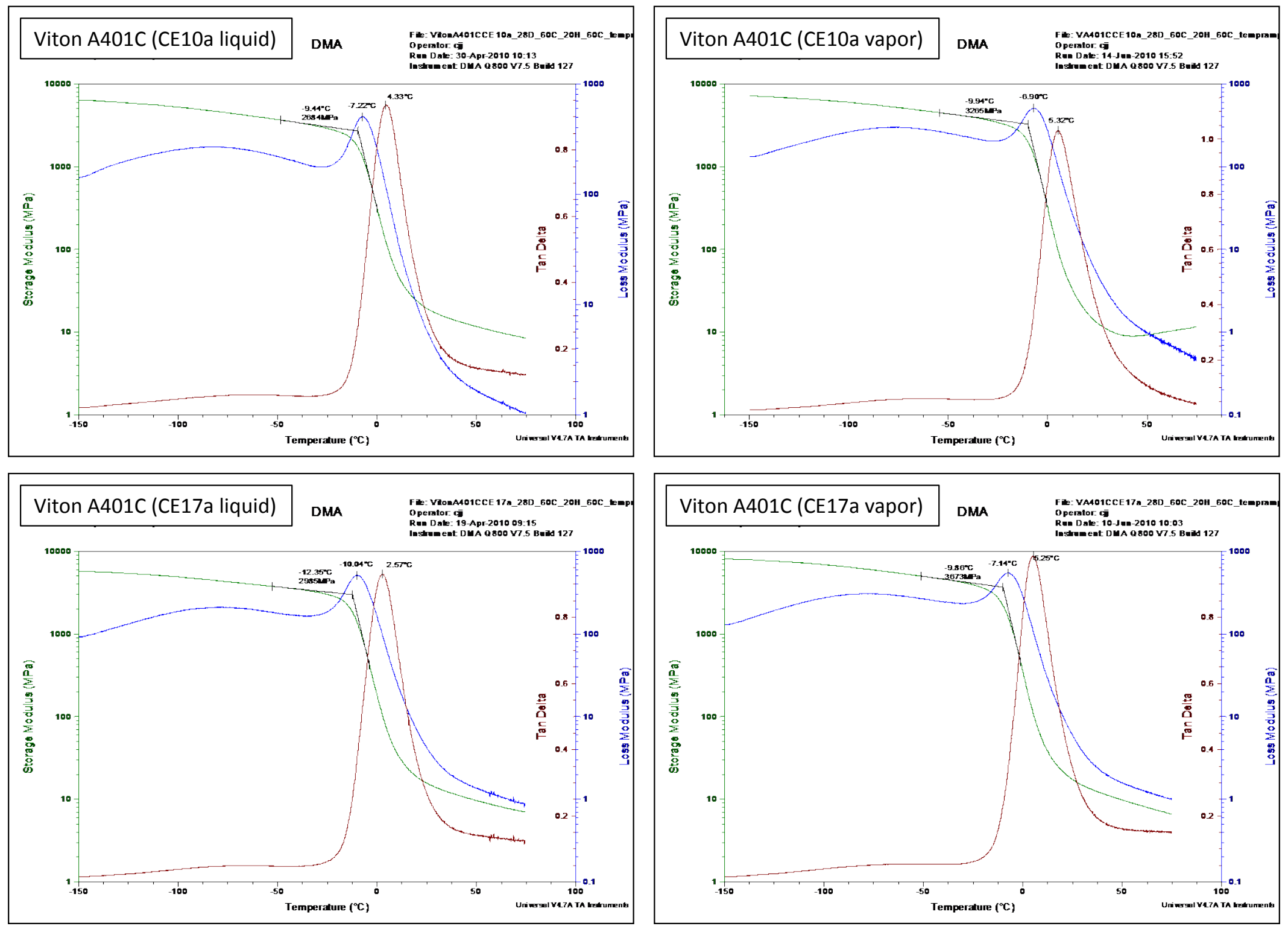
Viton A401C DMA Results for CE25a and CiBu16a Exposures
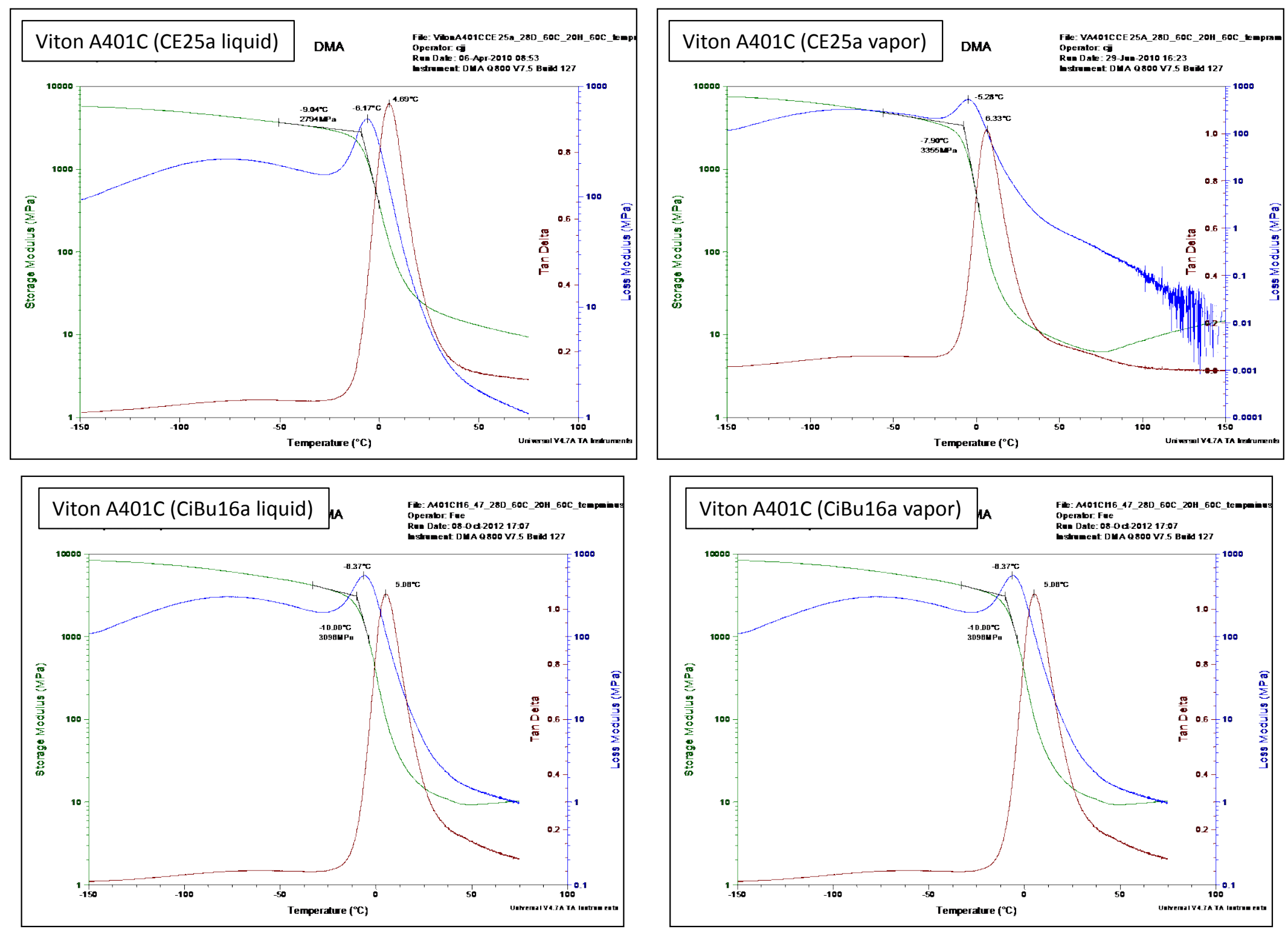
Viton A401C DMA Results for CiBu24a Exposures
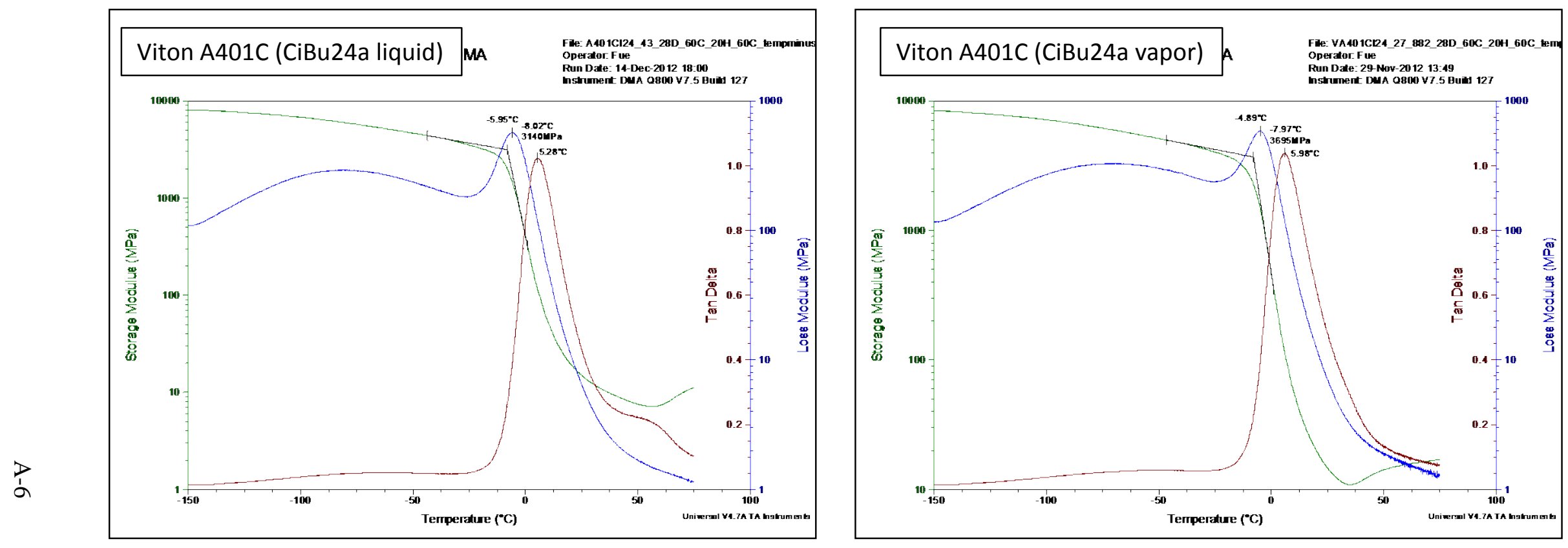


\section{Viton B601C DMA Results for Control and Fuel C Exposures}
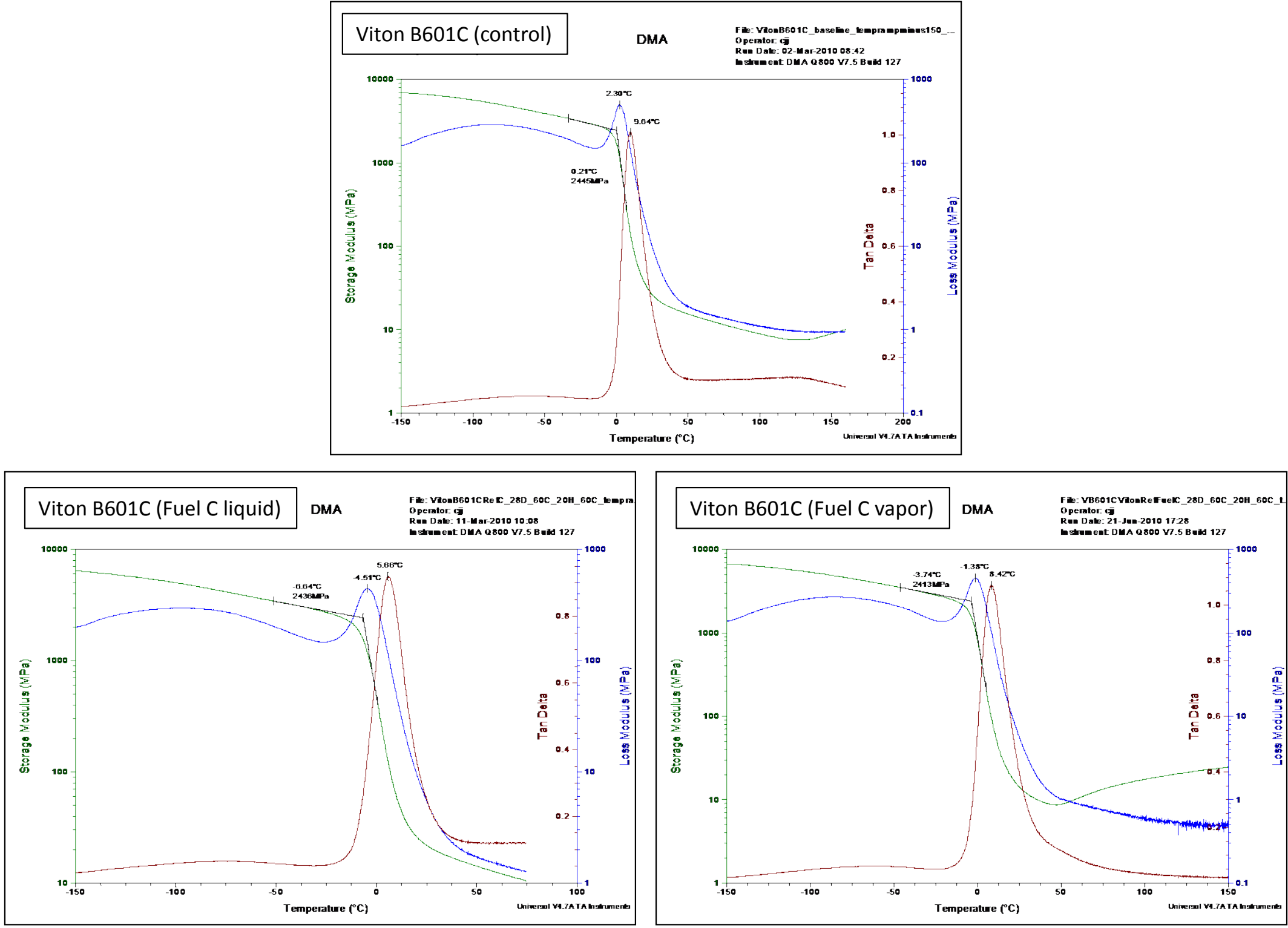
Viton B601C DMA Results for CE10a and CE17a Exposures
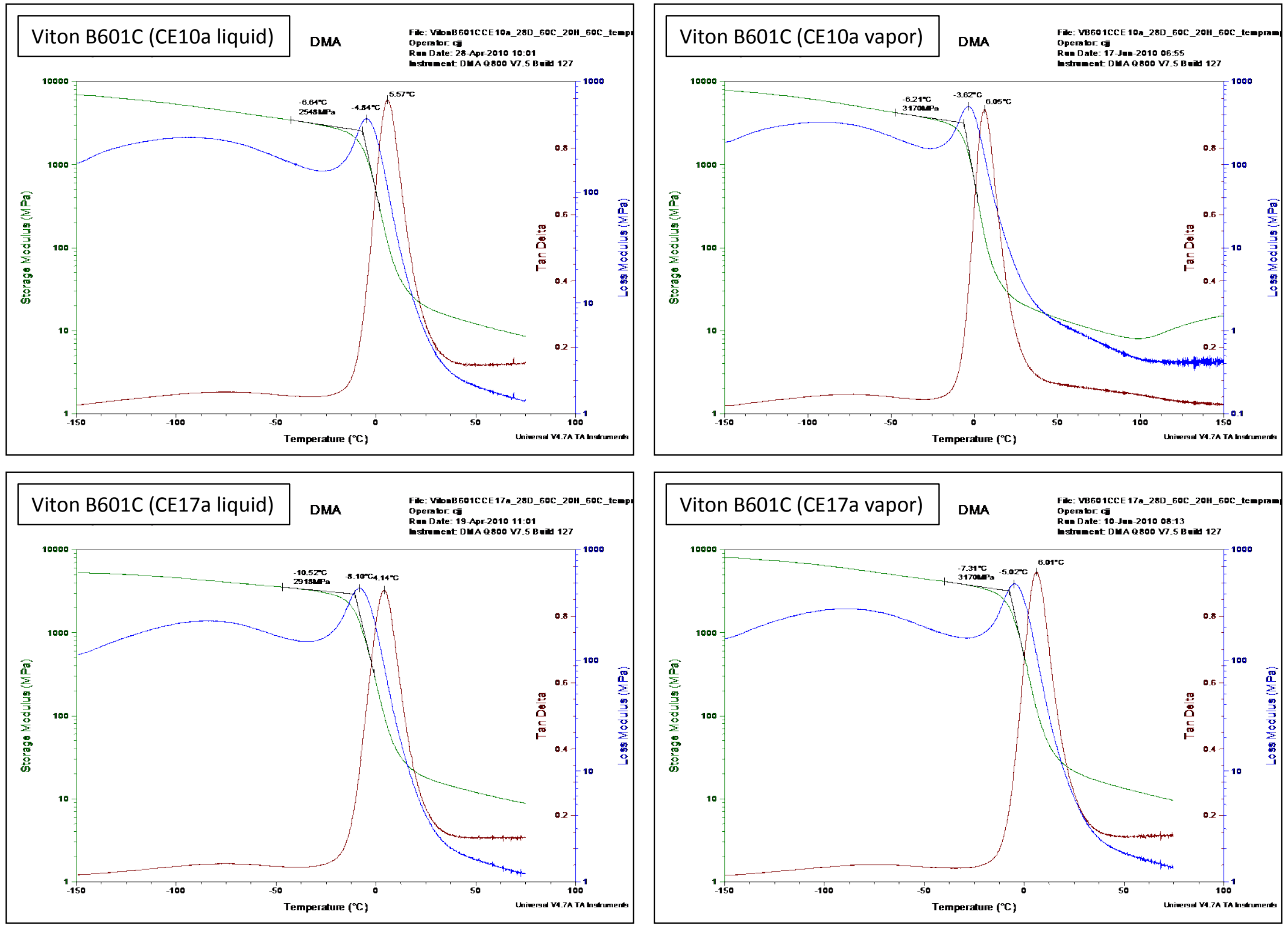
Viton B601C DMA Results for CE25a and CiBu16a Exposures
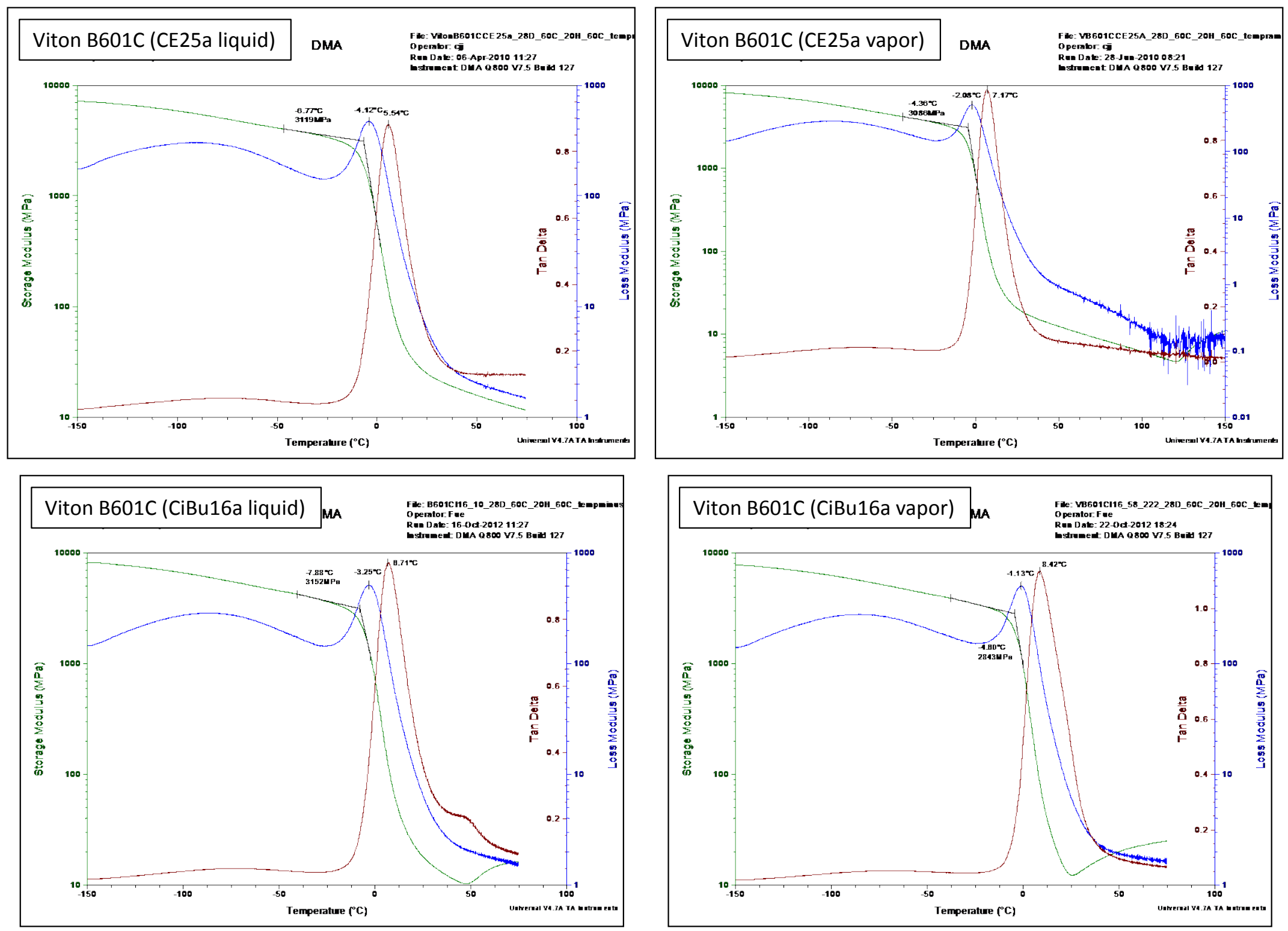
Viton B601C DMA Results for CE25a and CiBu16a Exposures

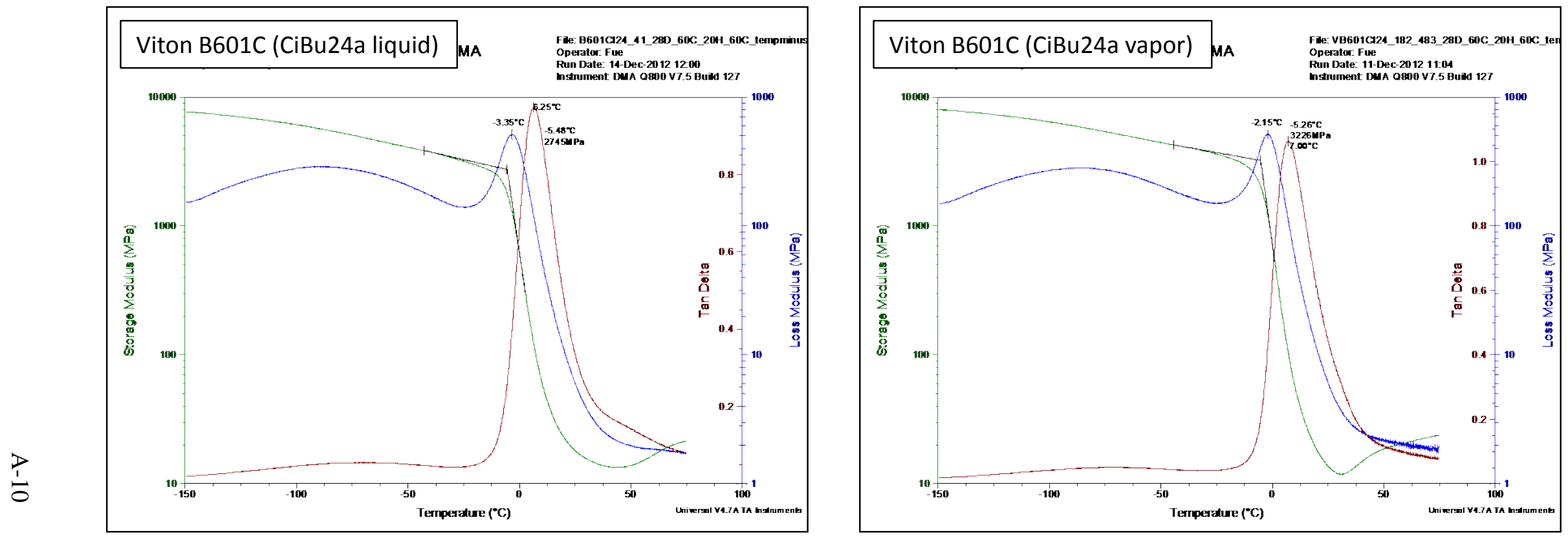


Fluorosilicone DMA Results for Control and Fuel C Exposures

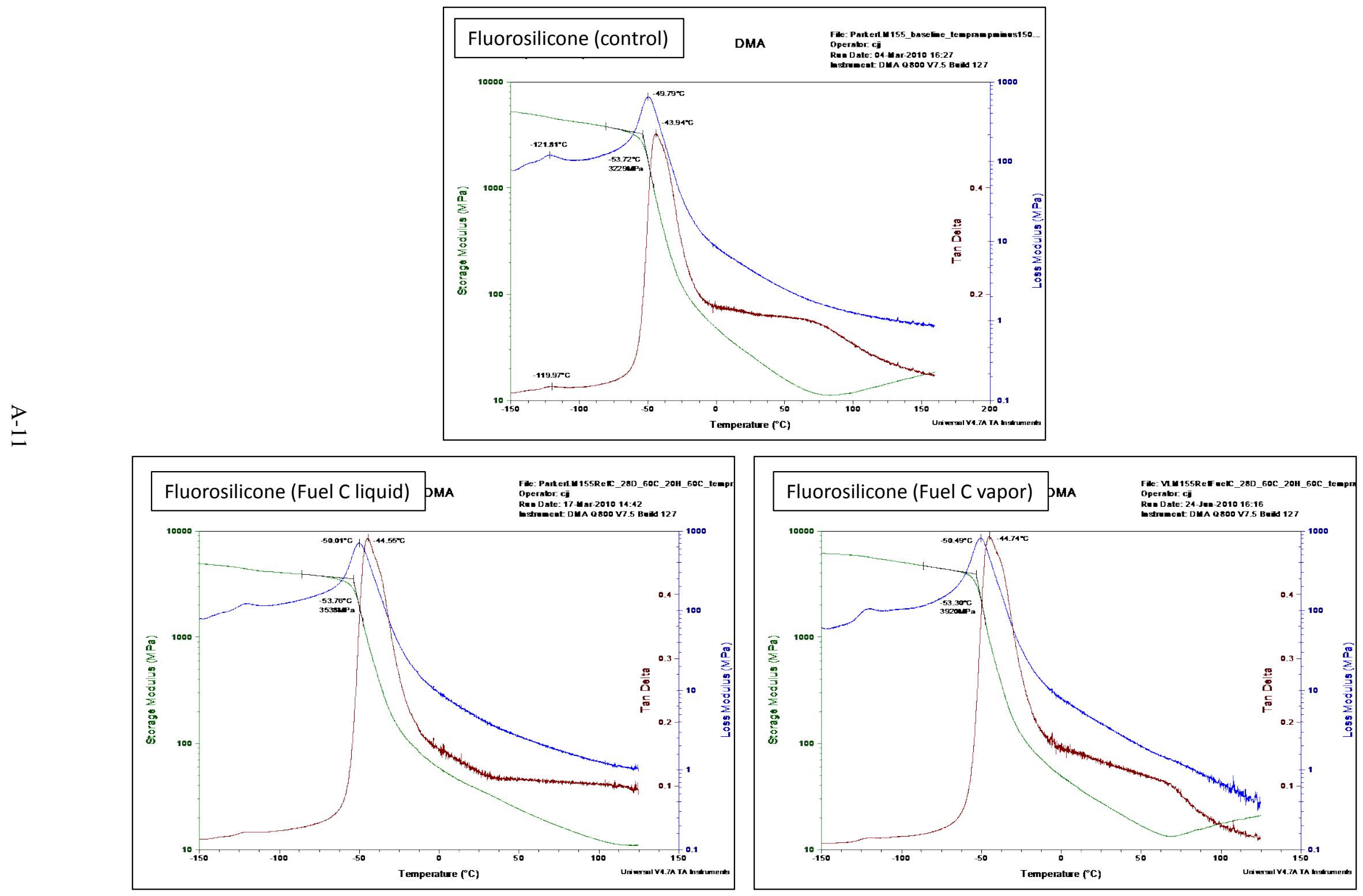




\section{Fluorosilicone DMA Results for CE10a and CE17a Exposures}
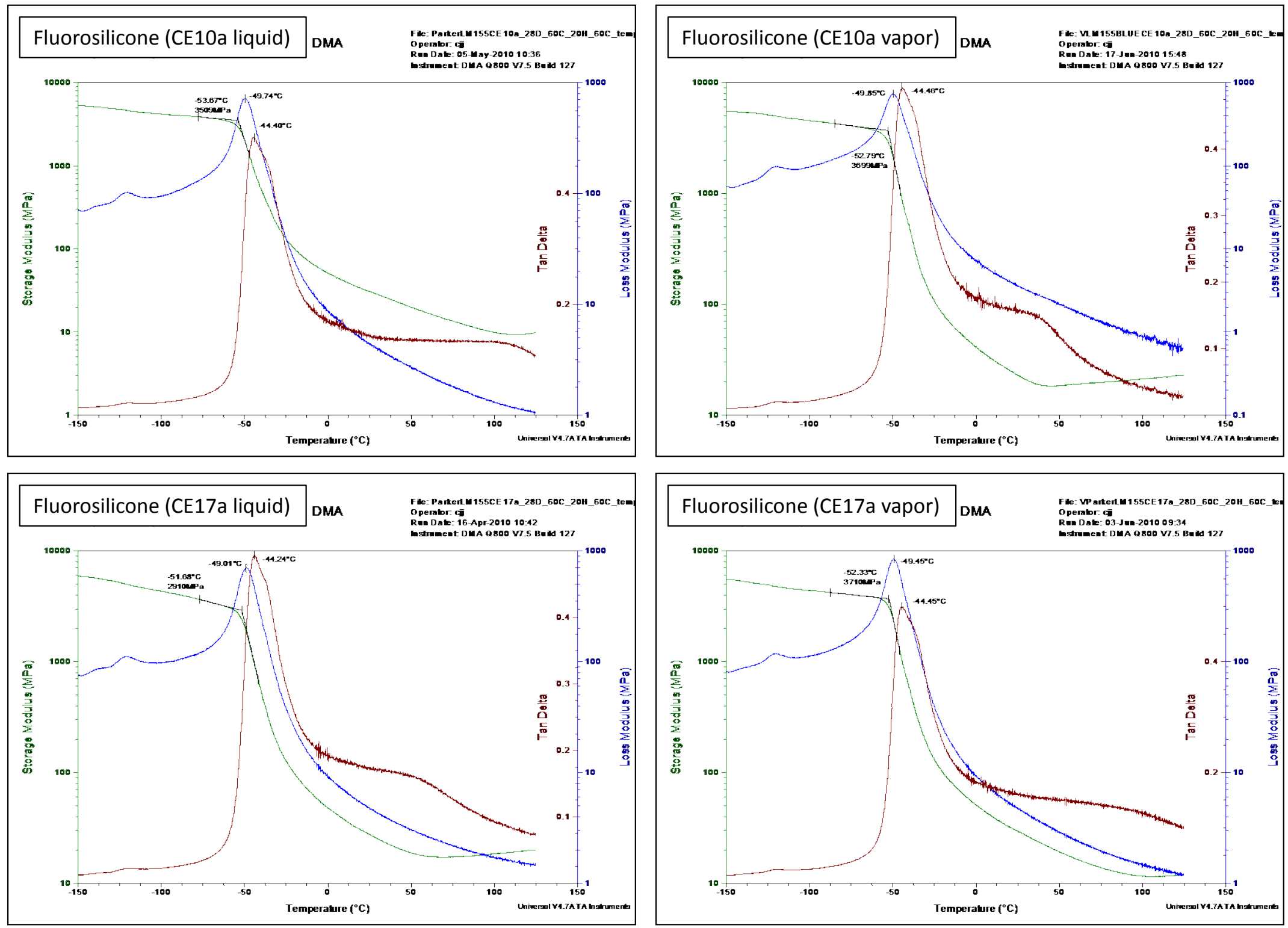


\section{Fluorosilicone DMA Results for CE25a and CiBu16a Exposures}
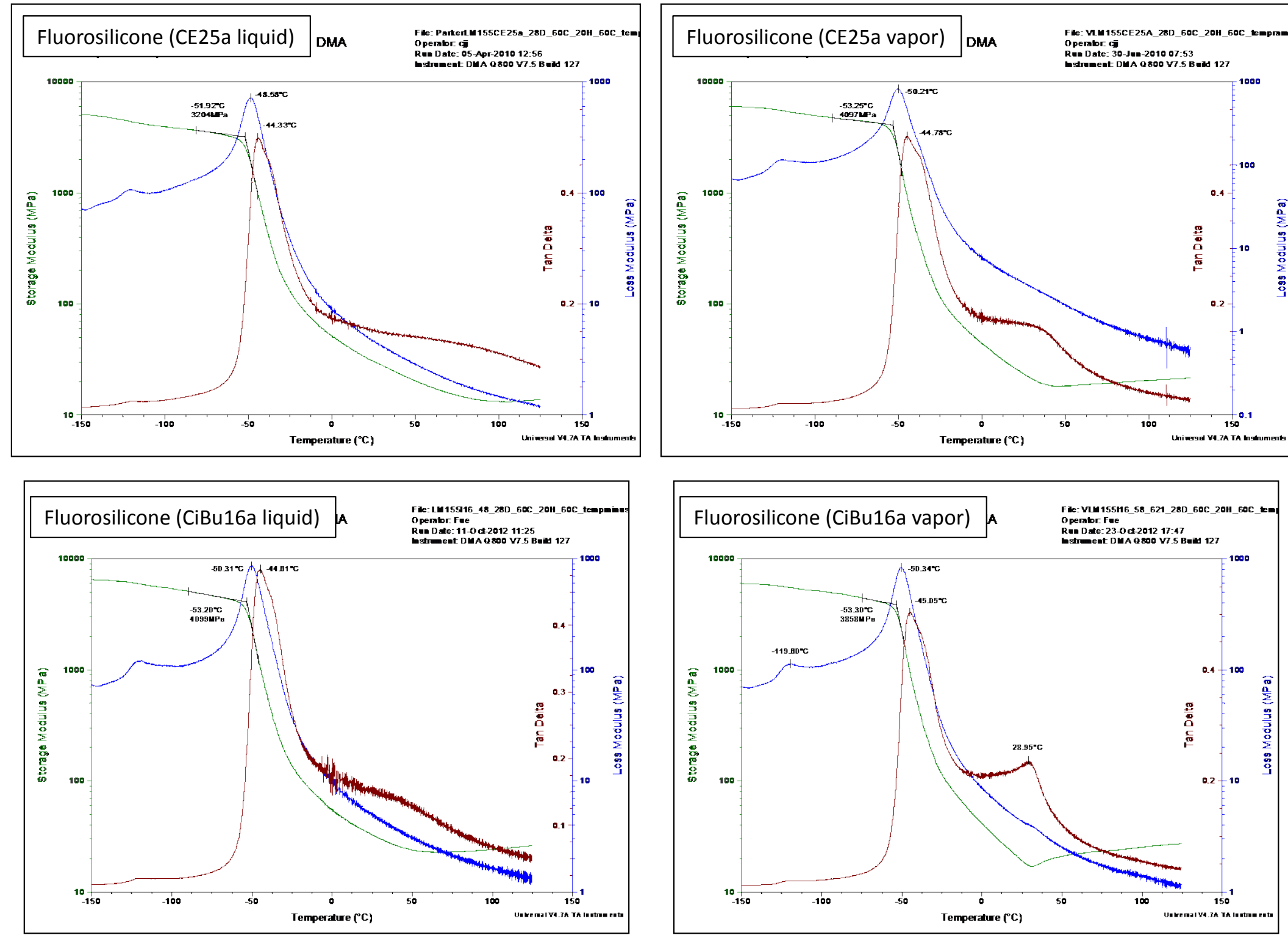


\section{Fluorosilicone DMA Results for CiBu24a Exposures}
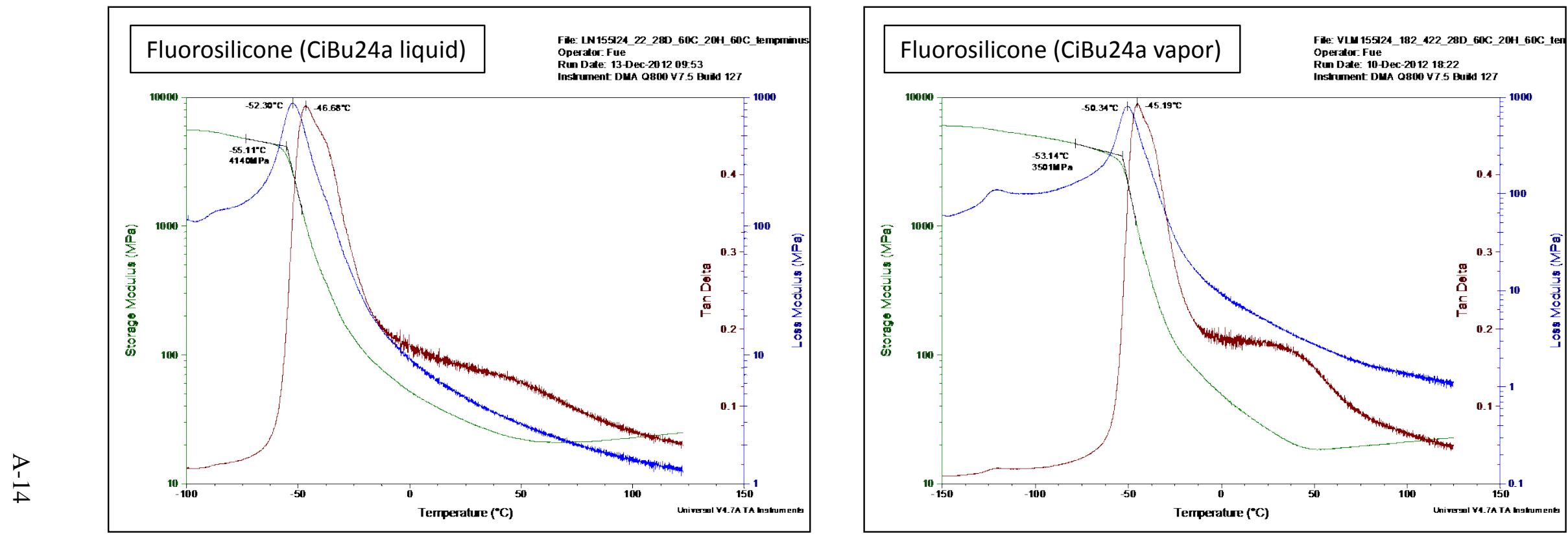
NBR\#1 DMA Results for Control and Fuel C Exposures
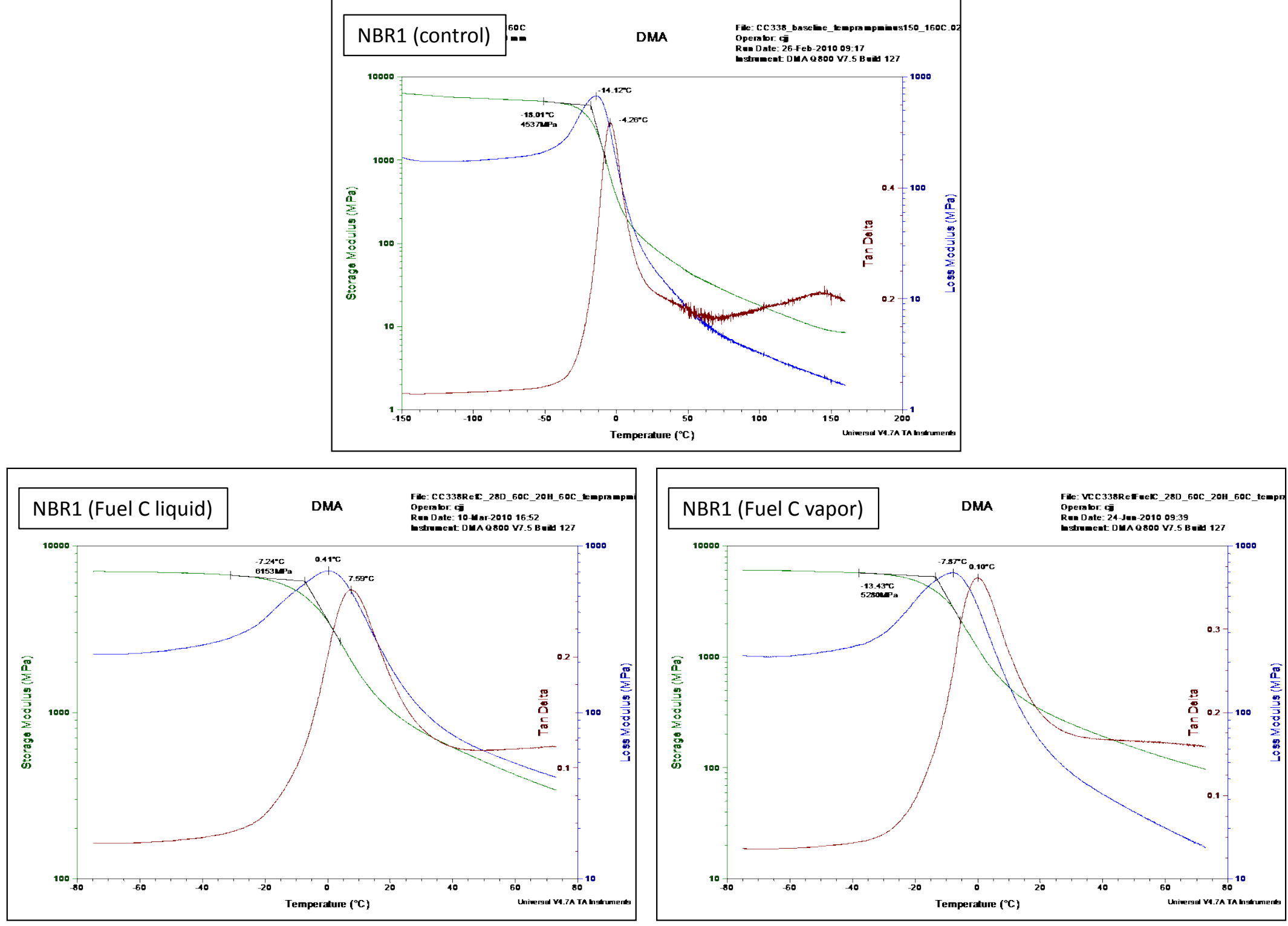


\section{NBR\#1 DMA Results for CE10a and CE17a Exposures}
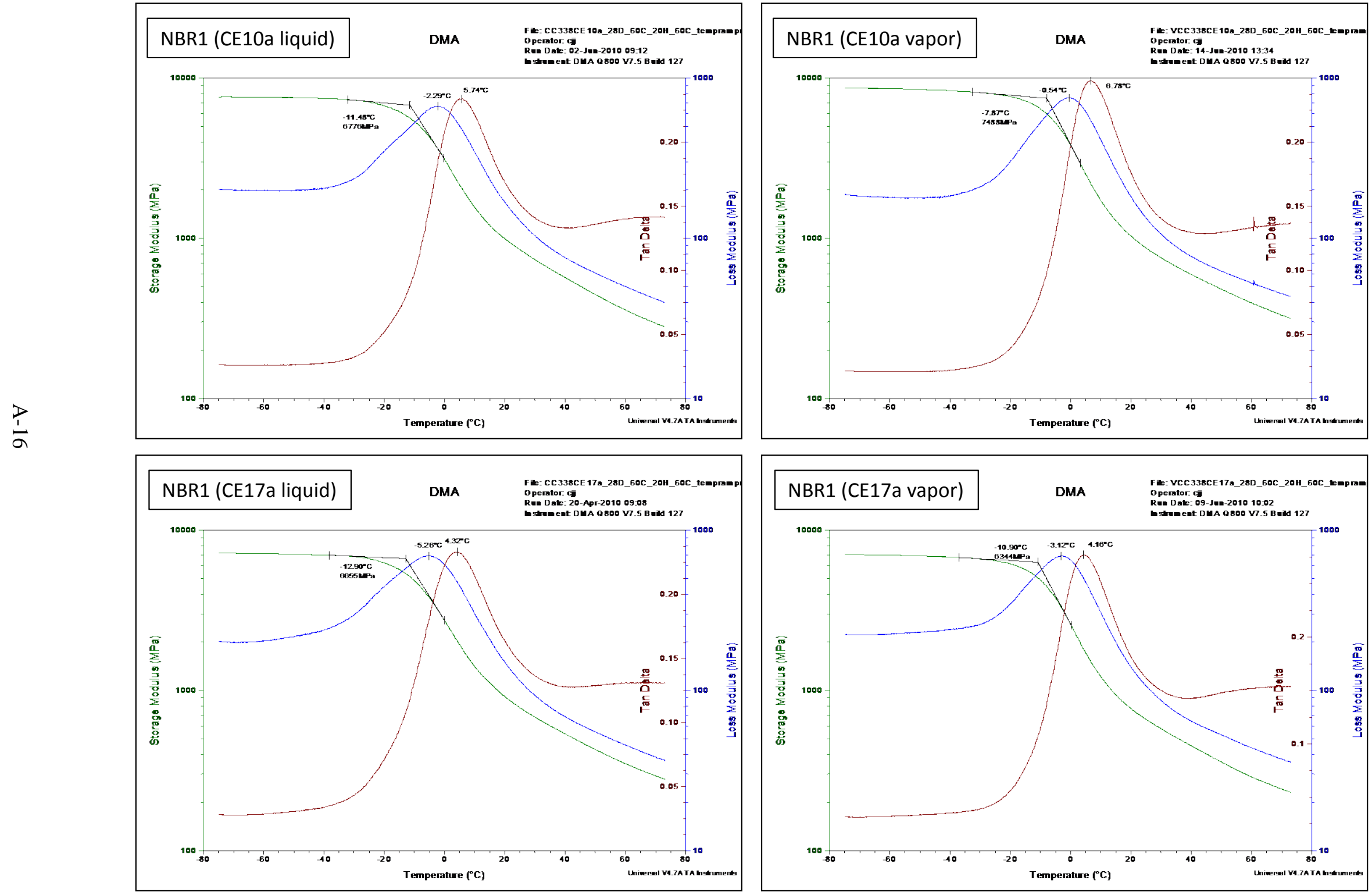
NBR\#1 DMA Results for CE25a and CiBu16a Exposures
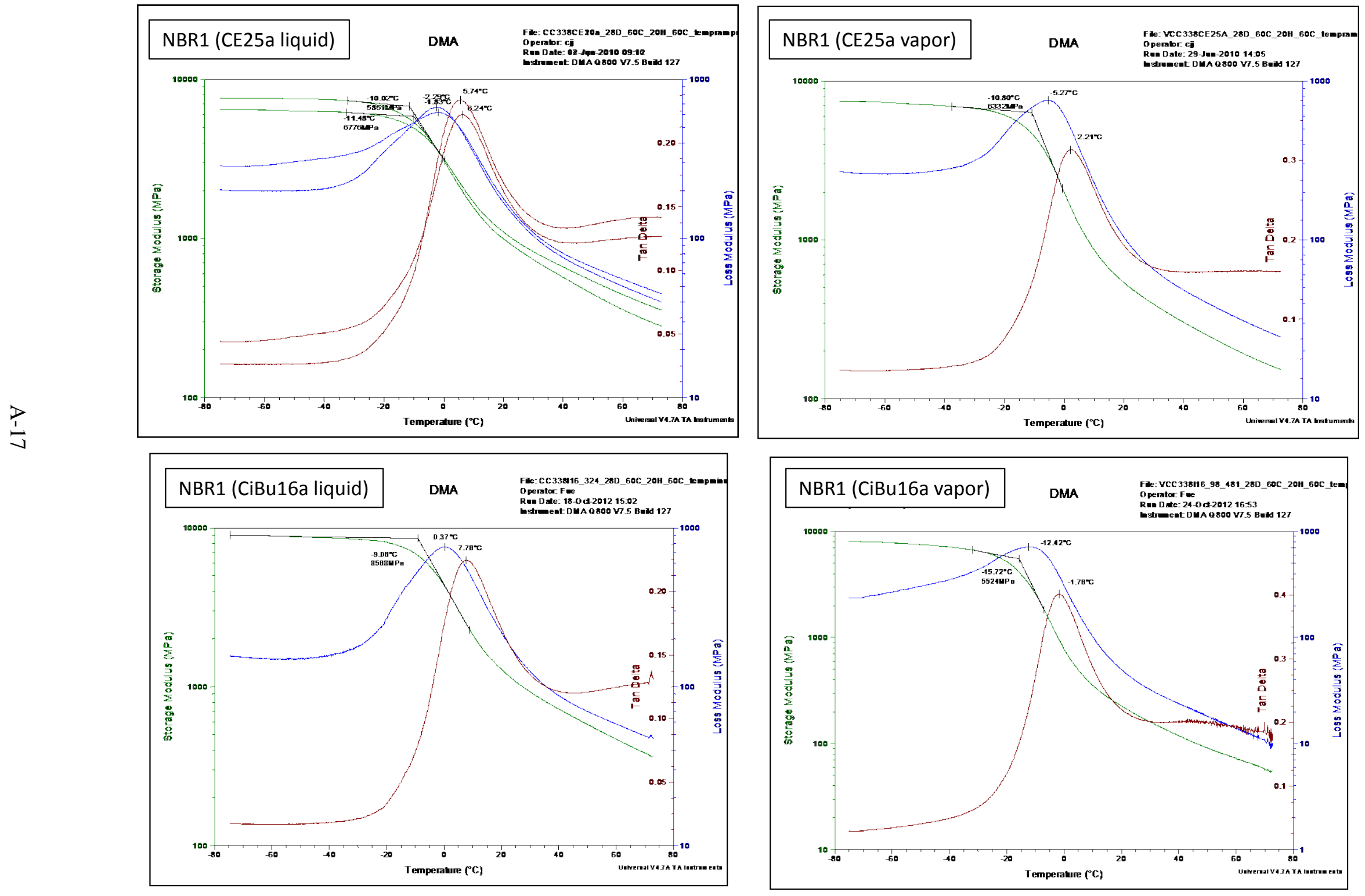


\section{NBR\#1 DMA Results for CiBu24a Exposures}
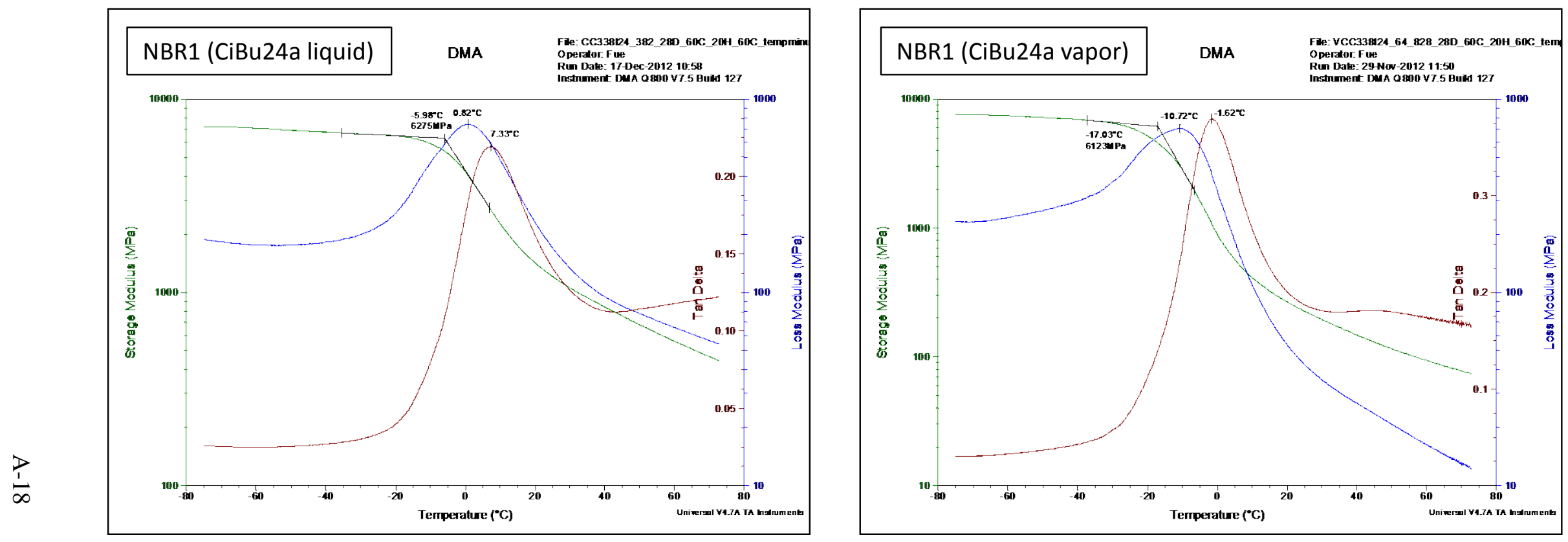
NBR\#2 DMA Results for Control and Fuel C Exposures
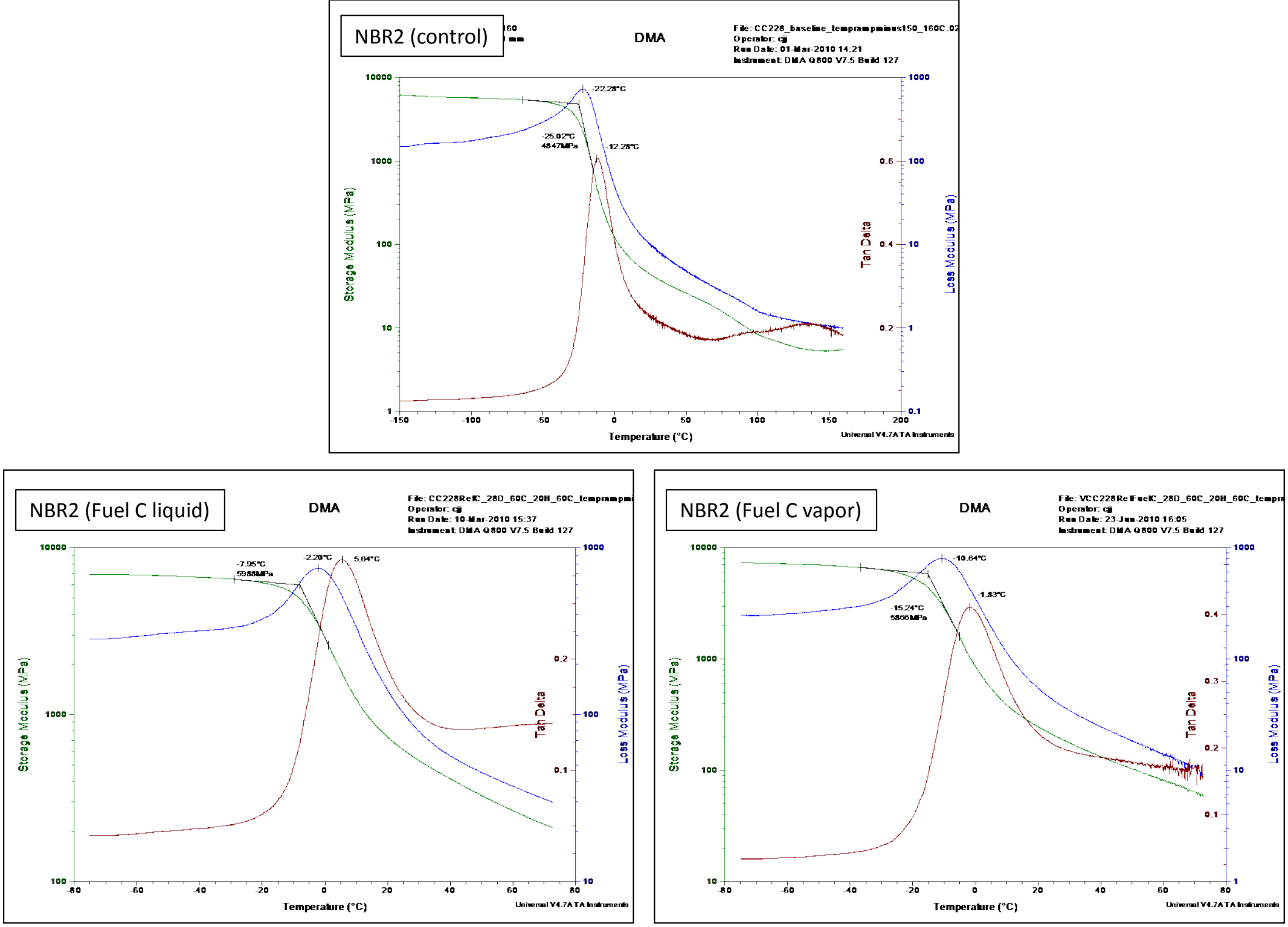


\section{NBR\#2 DMA Results for CE10a and CE17a Exposures}
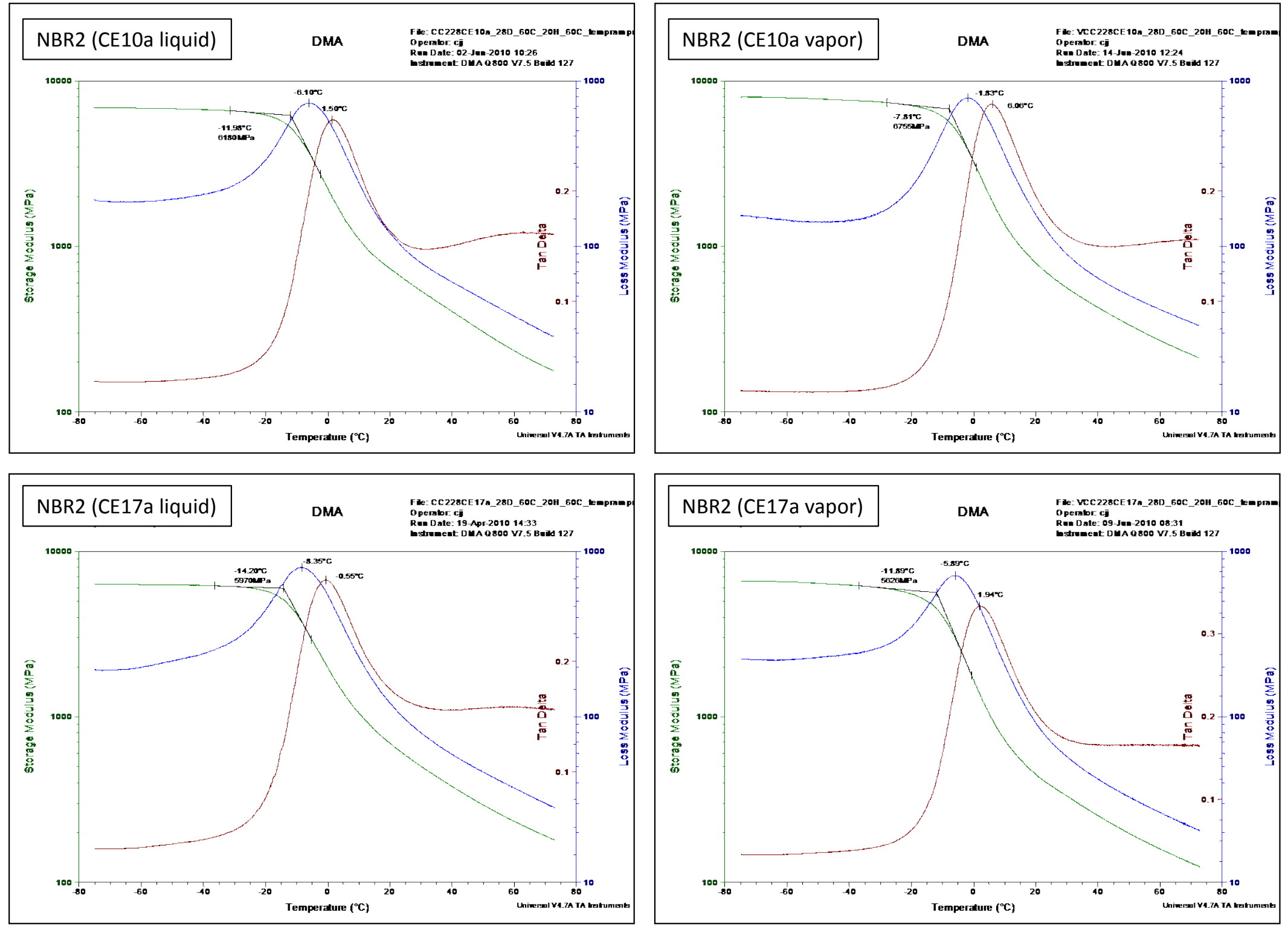
NBR\#2 DMA Results for CE25a and CiBu16a Exposures
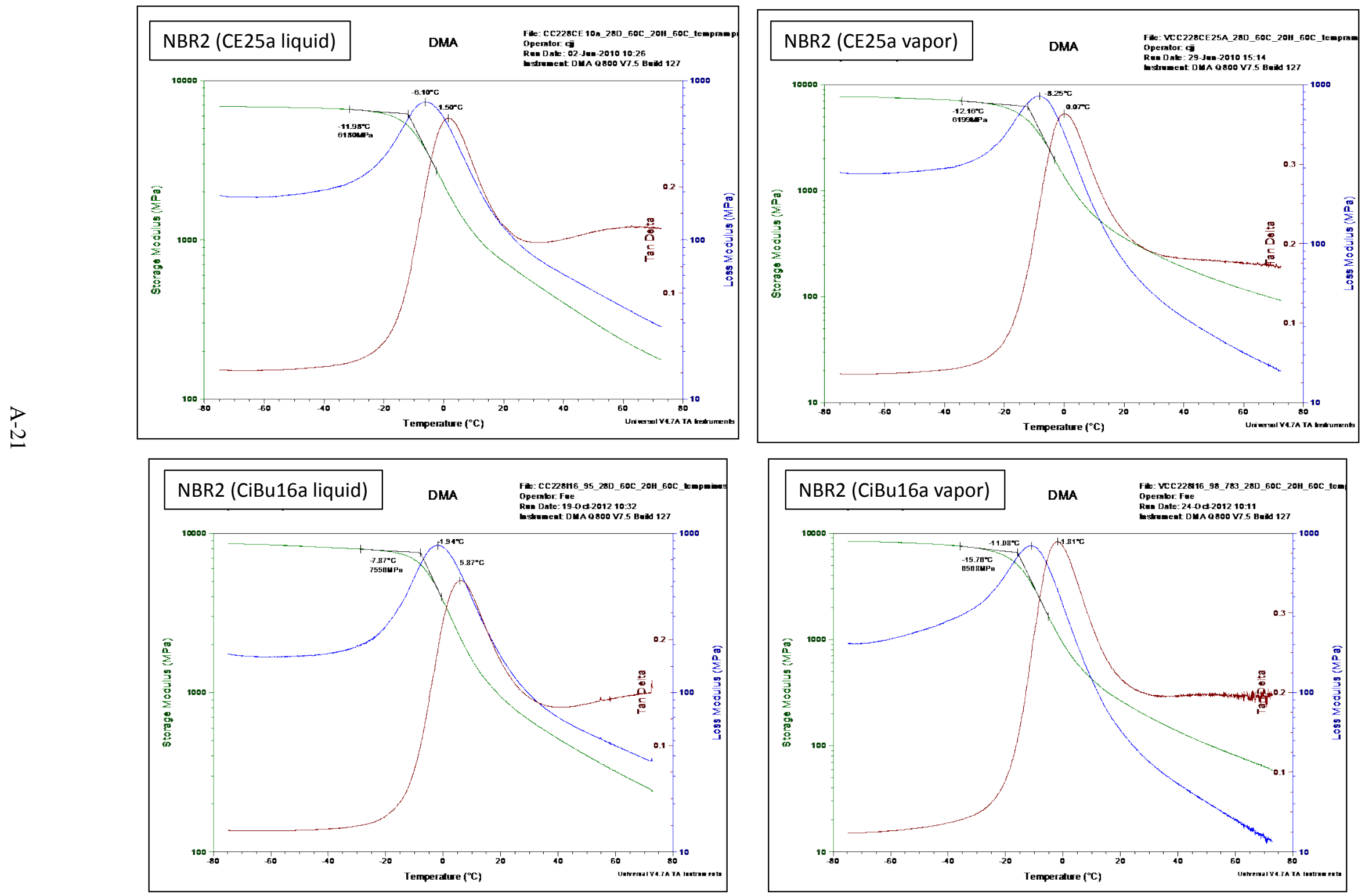


\section{NBR\#2 DMA Results for CiBu24a Exposures}
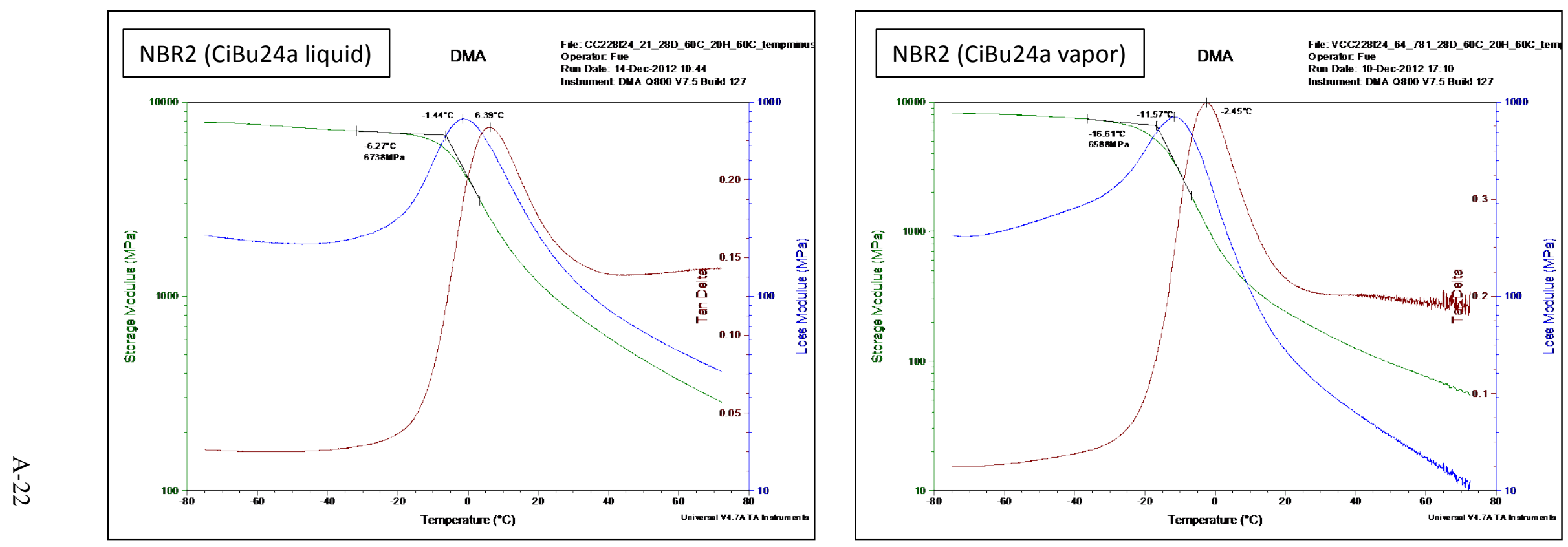
NBR\#3 DMA Results for Control and Fuel C Exposures
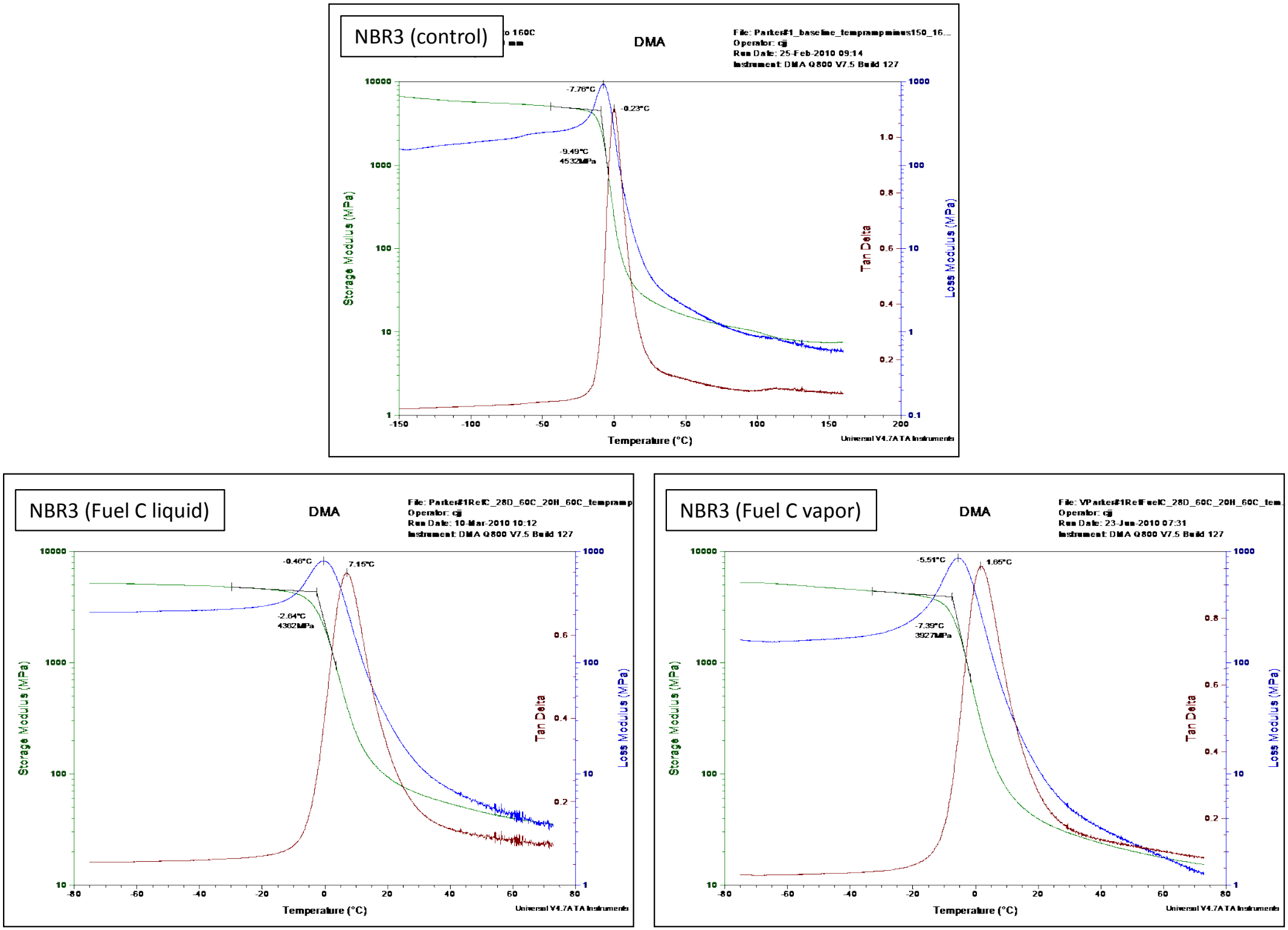


\section{NBR\#3 DMA Results for CE10a and CE17a Exposures}
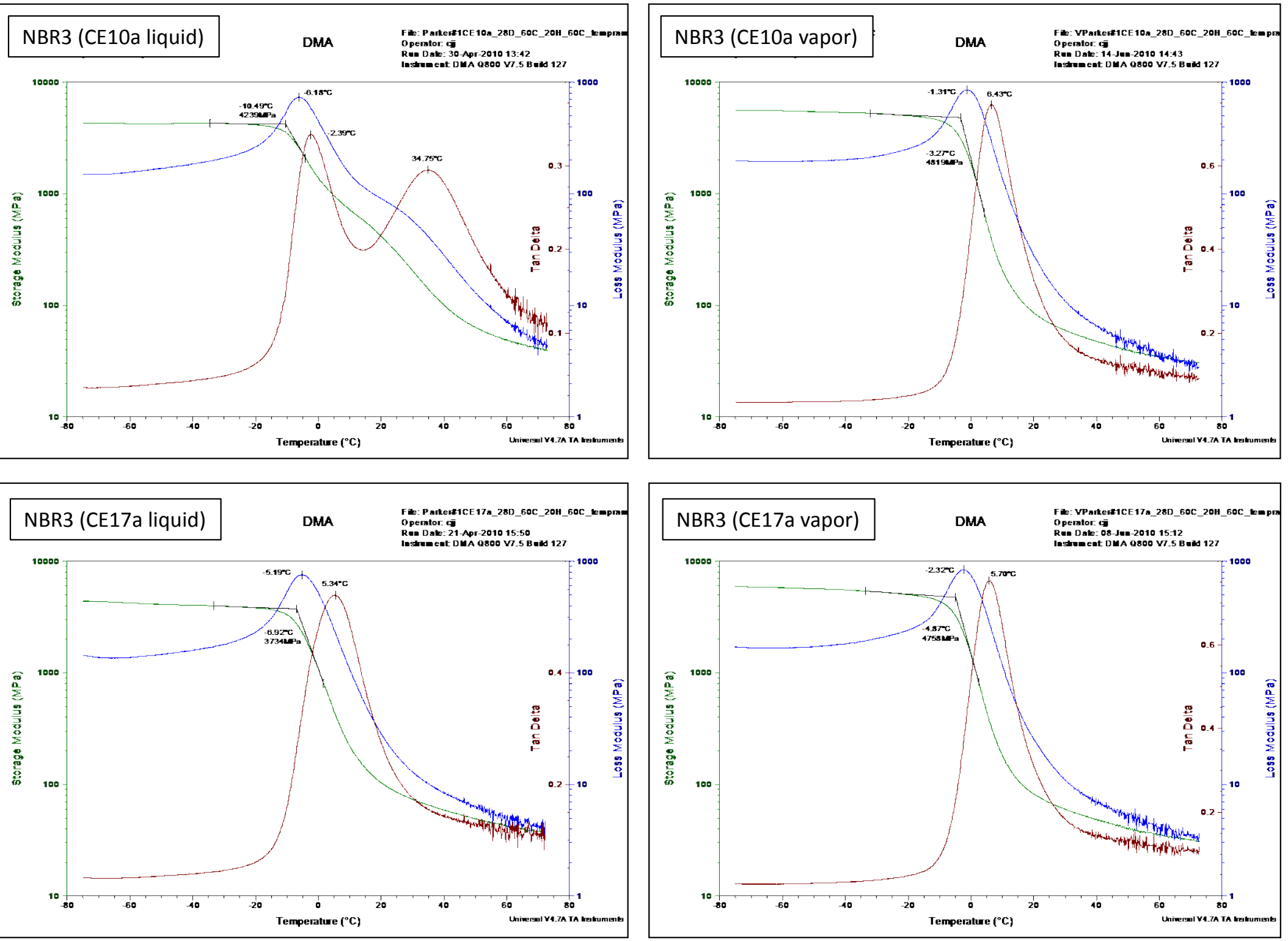
NBR\#3 DMA Results for CE25a and CiBu16a Exposures
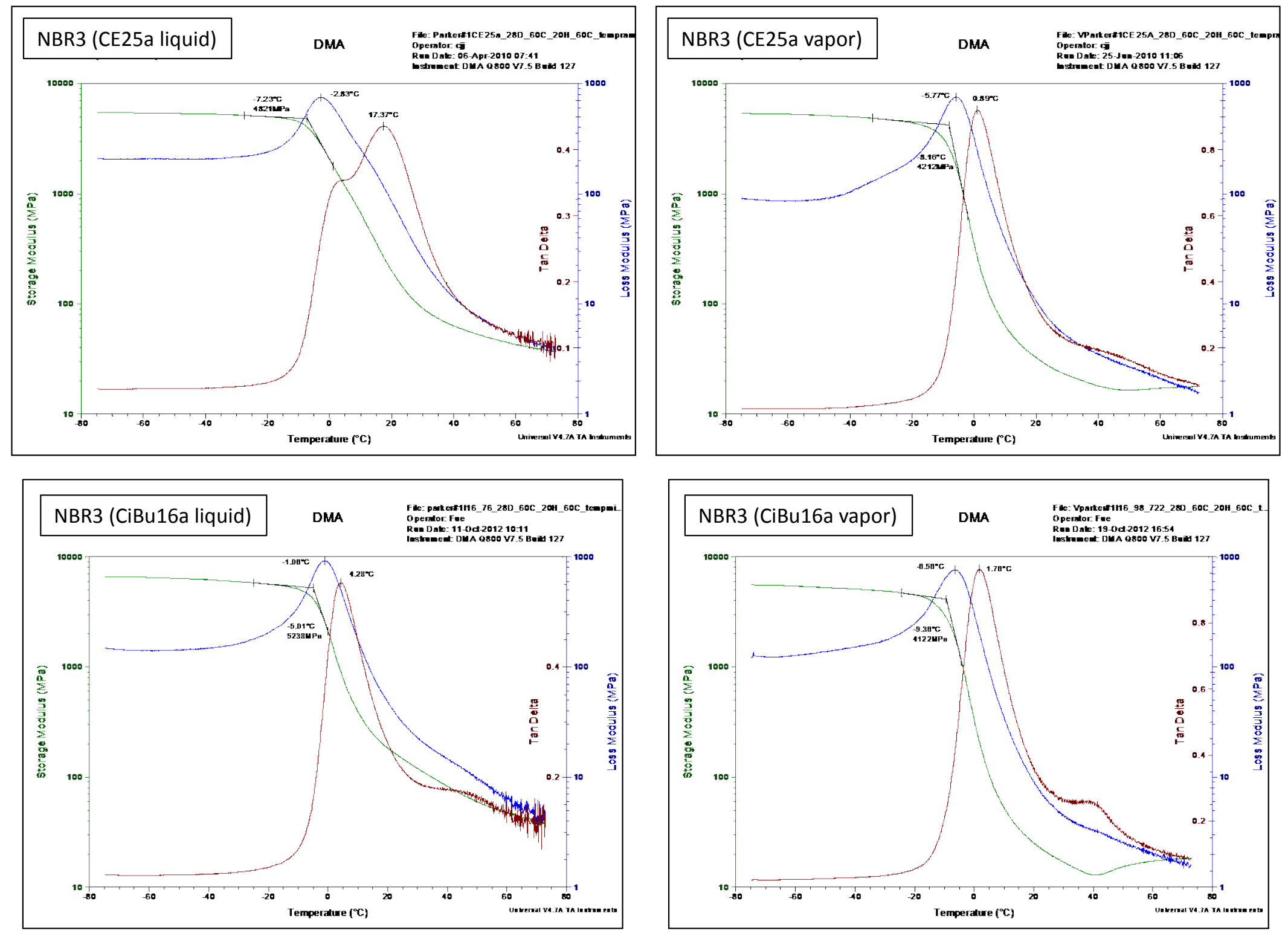


\section{NBR\#3 DMA Results for CiBu24a Exposures}

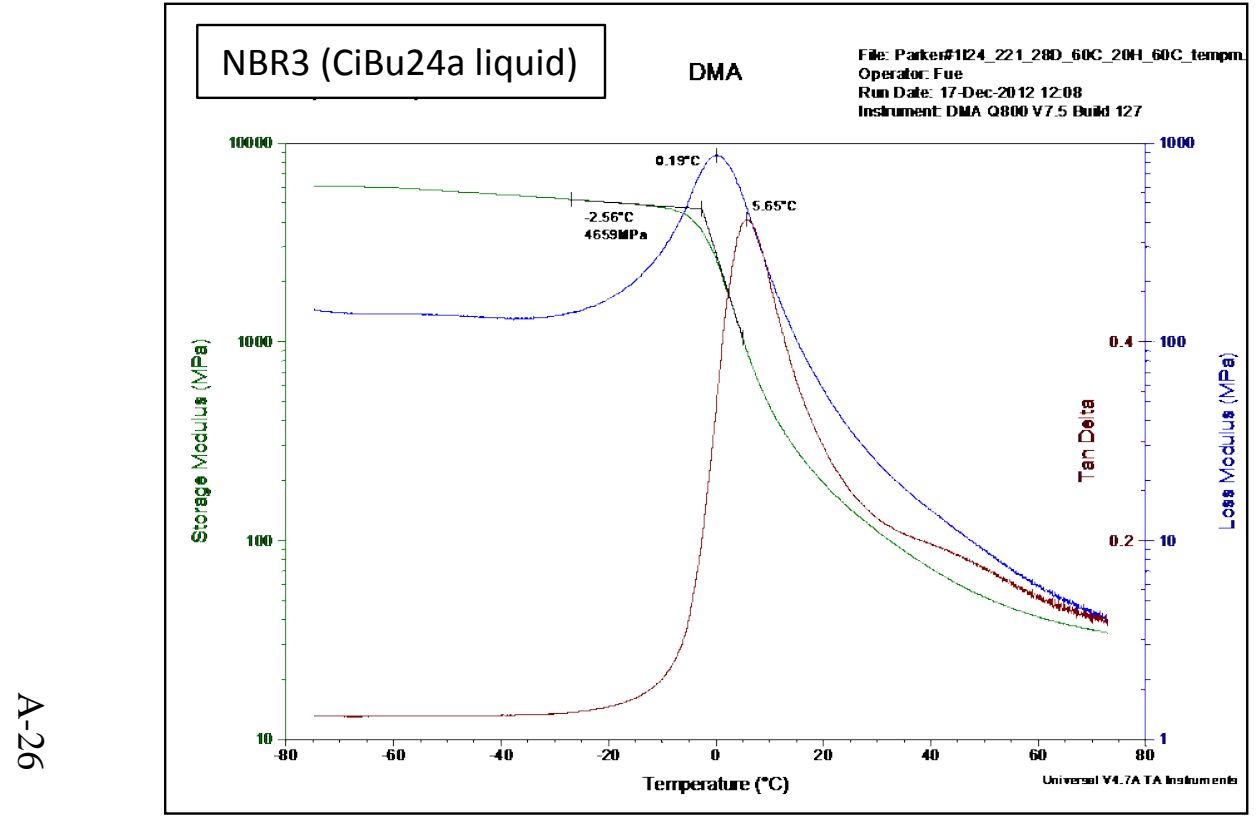

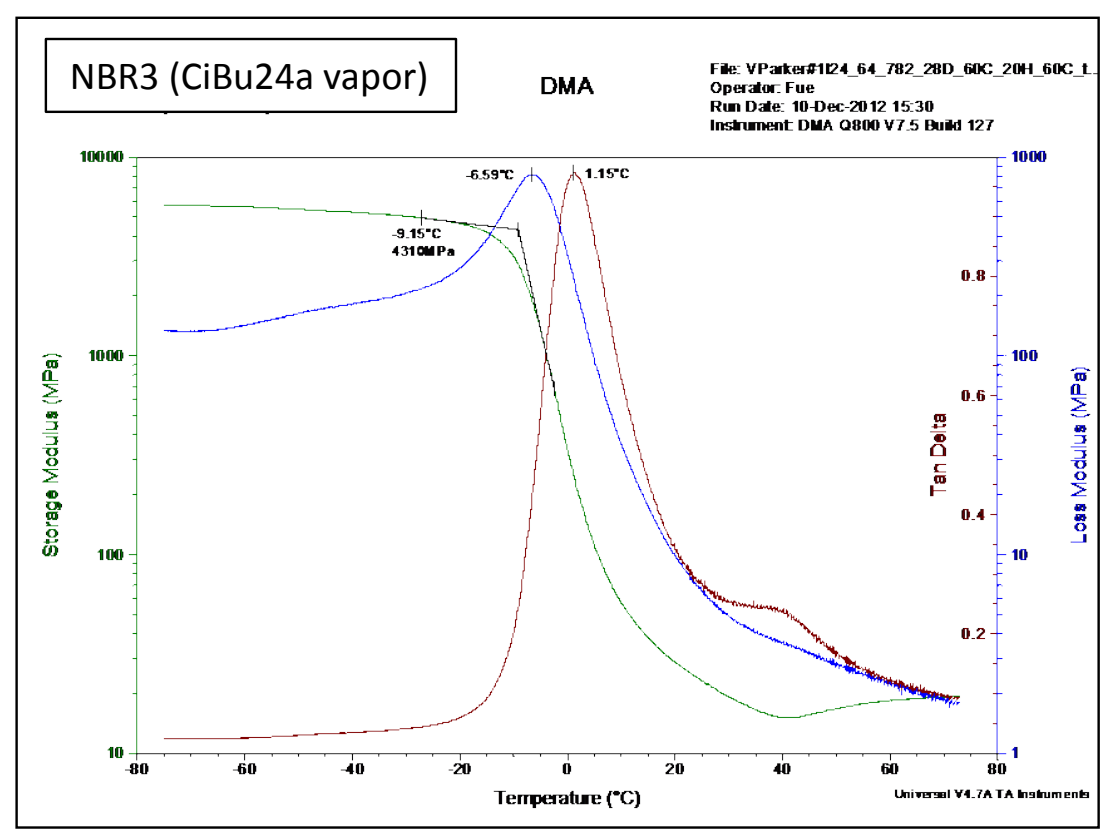


NBR\#4 DMA Results for Control and Fuel C Exposures
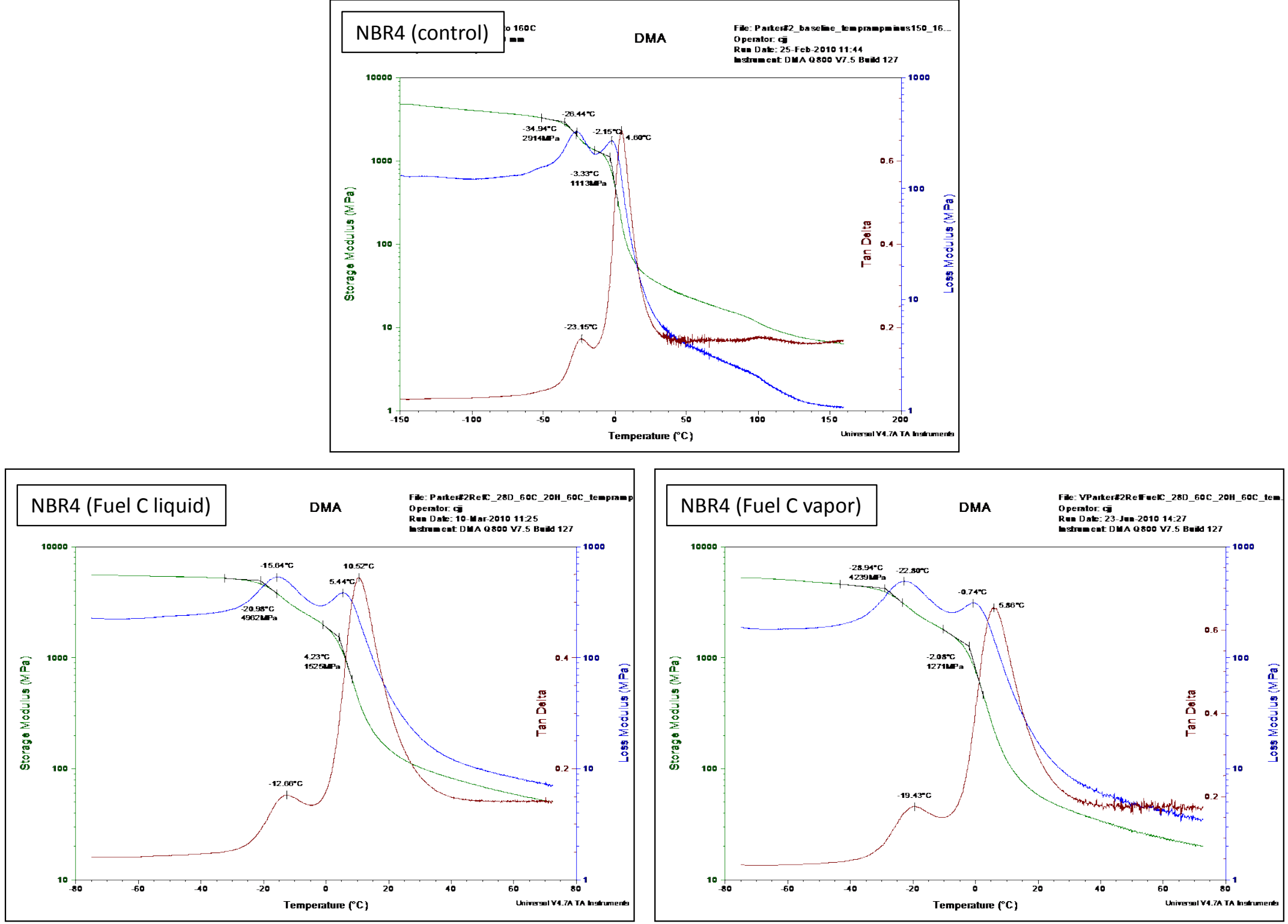
NBR\#4 DMA Results for CE10a and CE17a Exposures
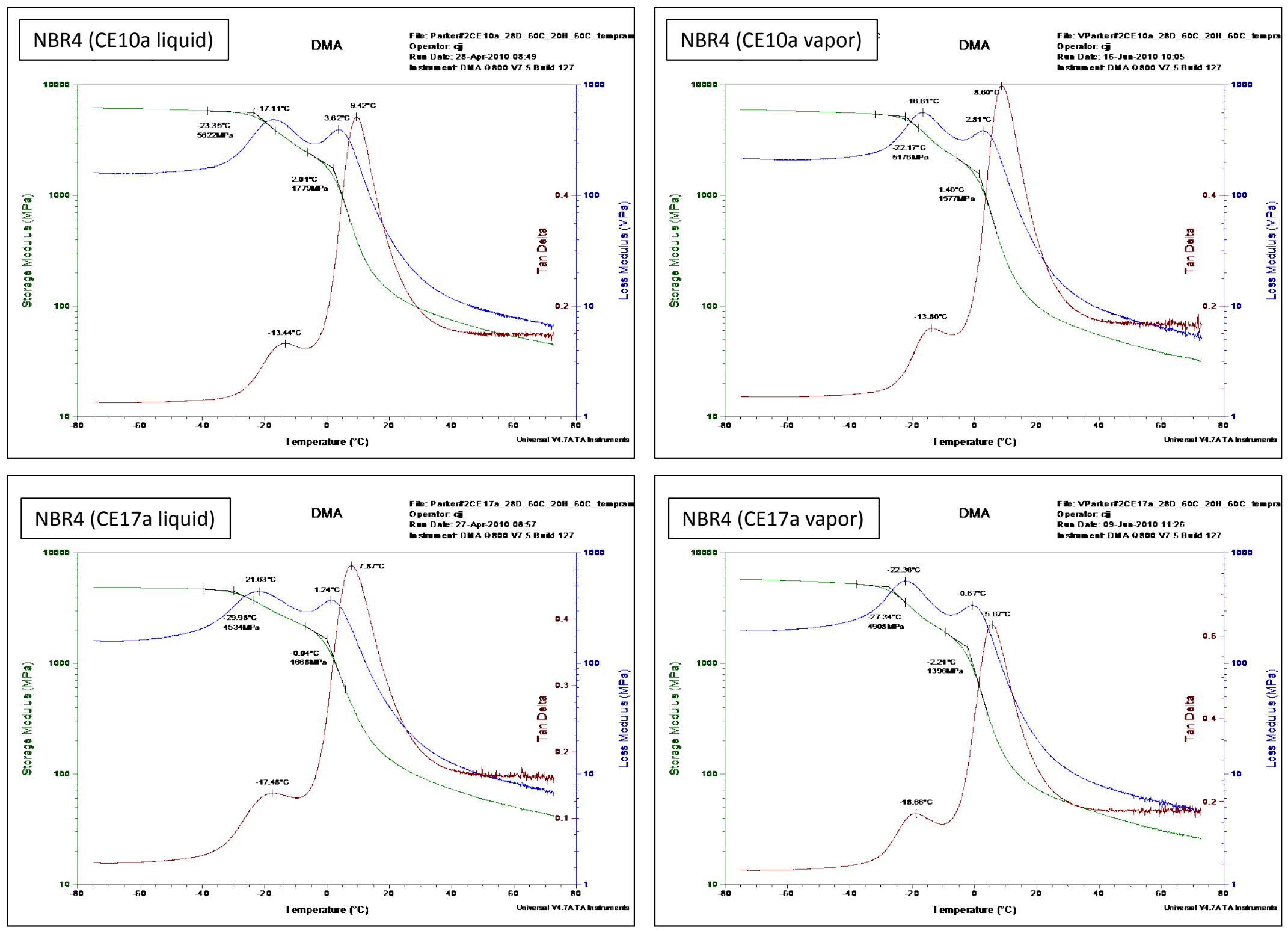
NBR\#4 DMA Results for CE25a and CiBu16a Exposures
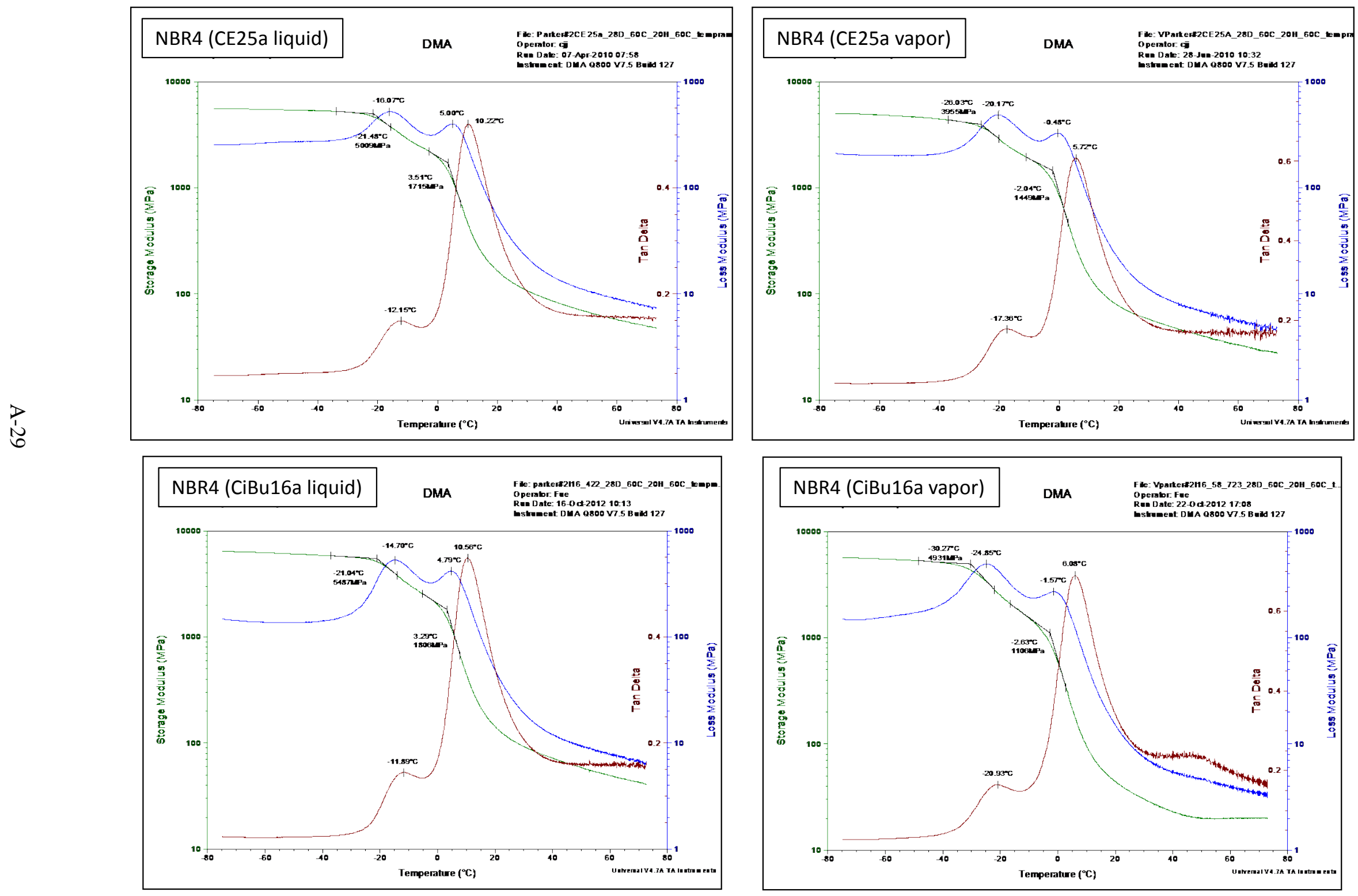


\section{NBR\#4 DMA Results for CiBu24a Exposures}

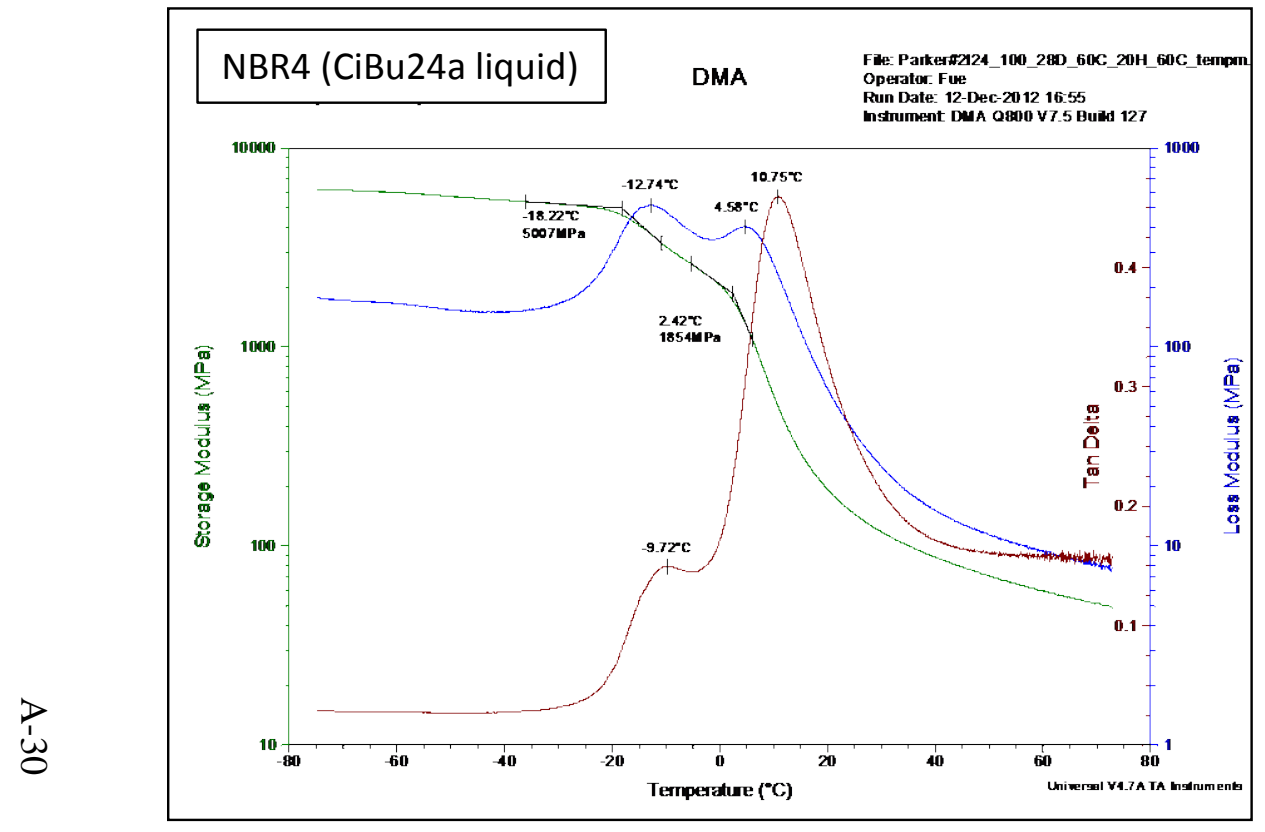

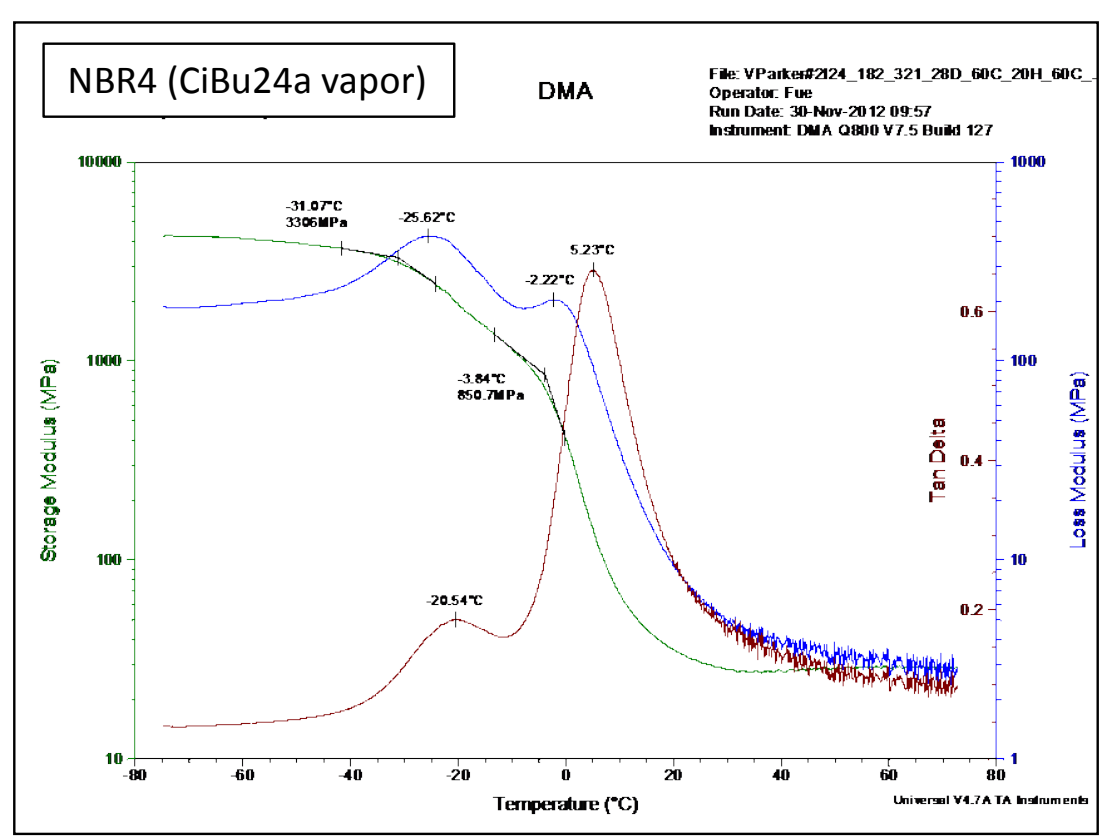


NBR\#5 DMA Results for Control and Fuel C Exposures
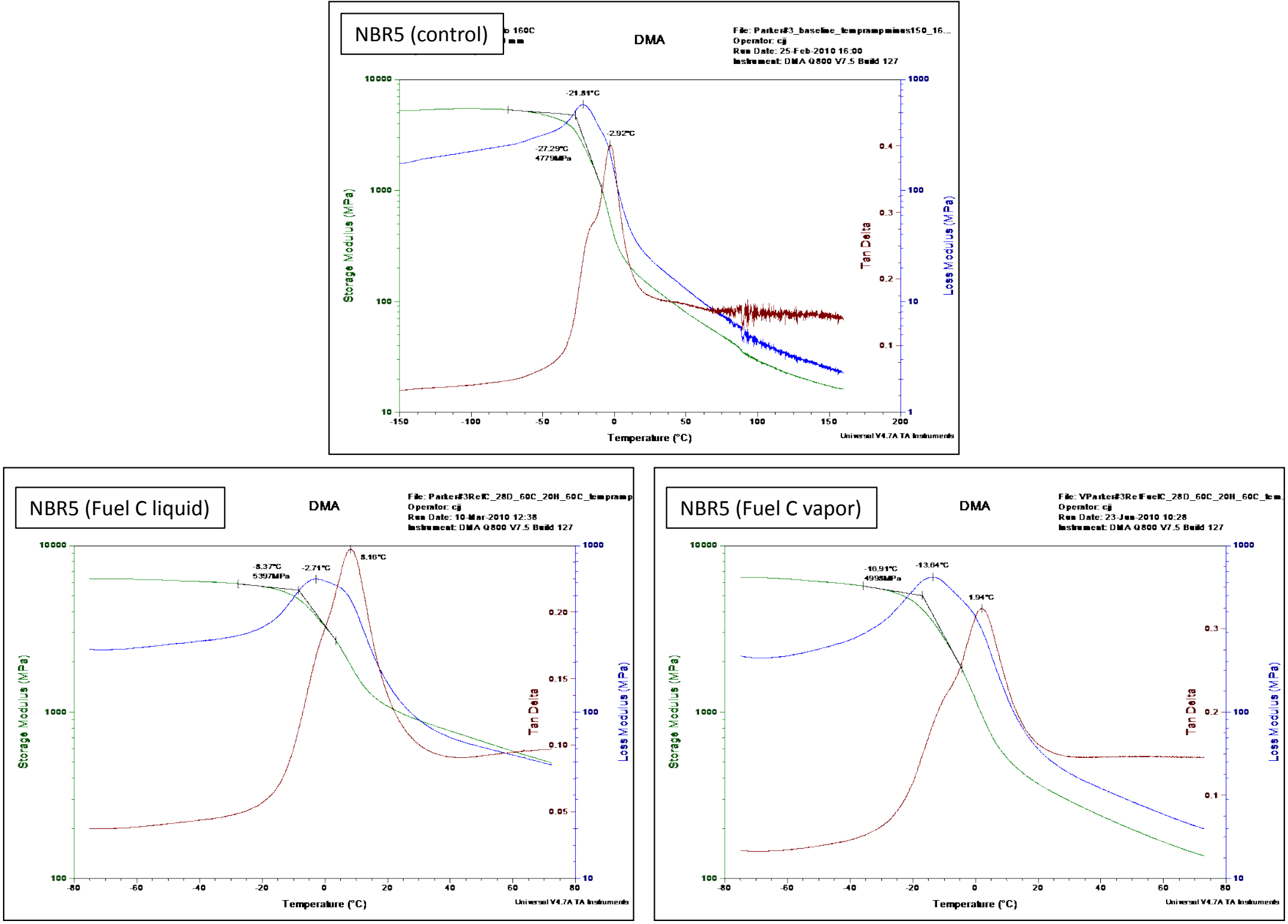


\section{NBR\#5 DMA Results for CE10a and CE17a Exposures}
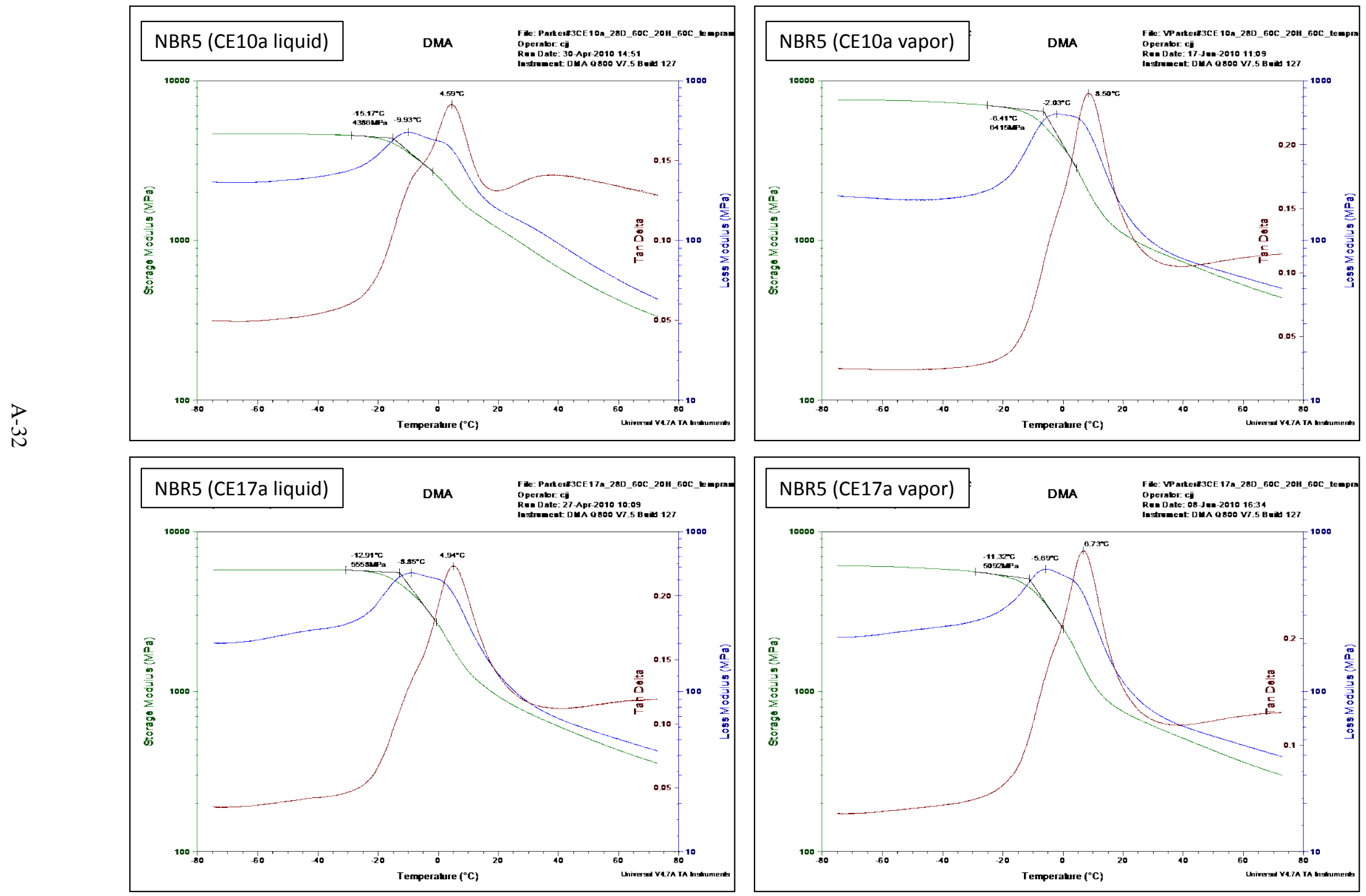
NBR\#5 DMA Results for CE25a and CiBu16a Exposures
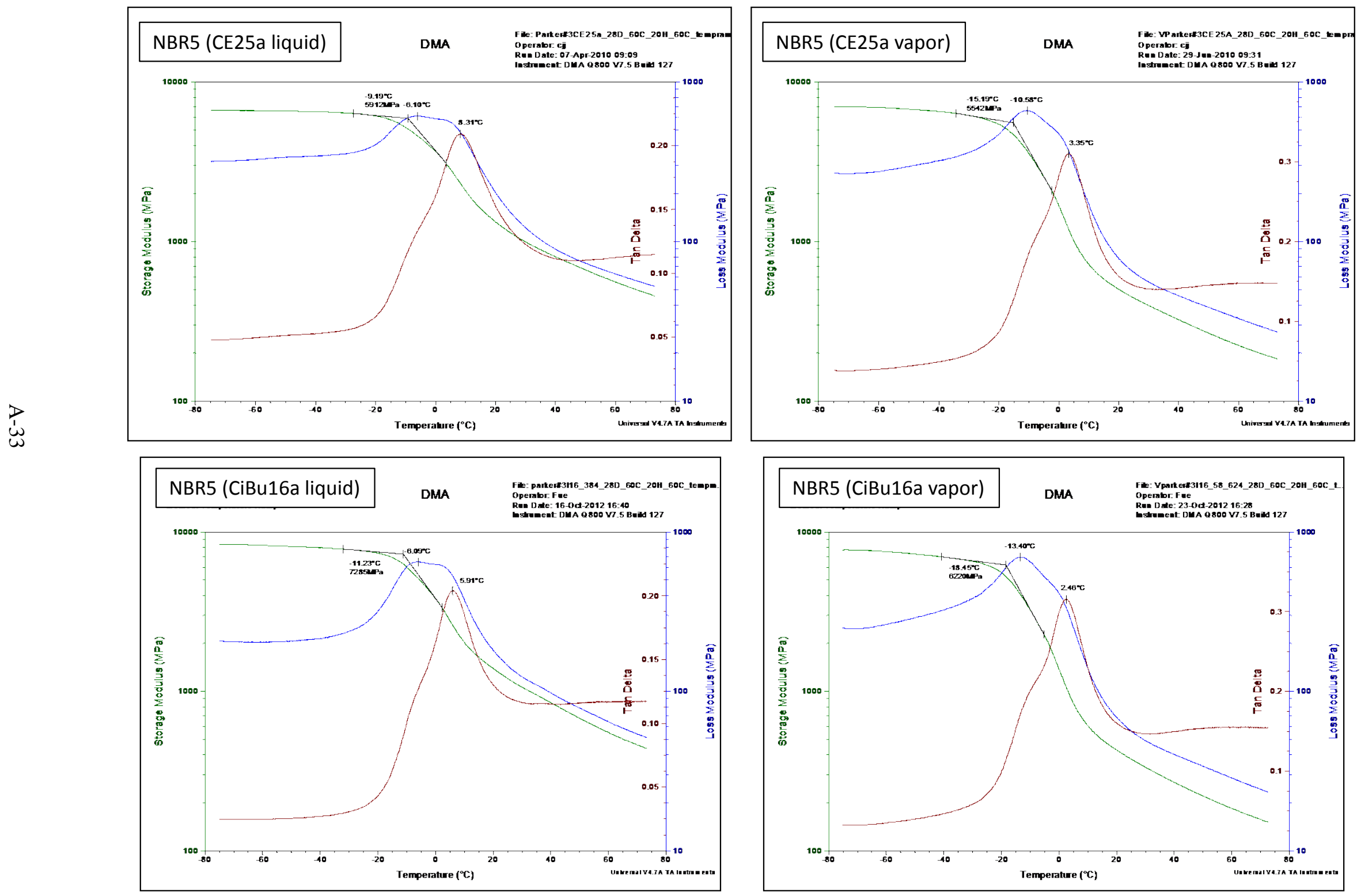


\section{NBR\#5 DMA Results for CiBu24a Exposures}

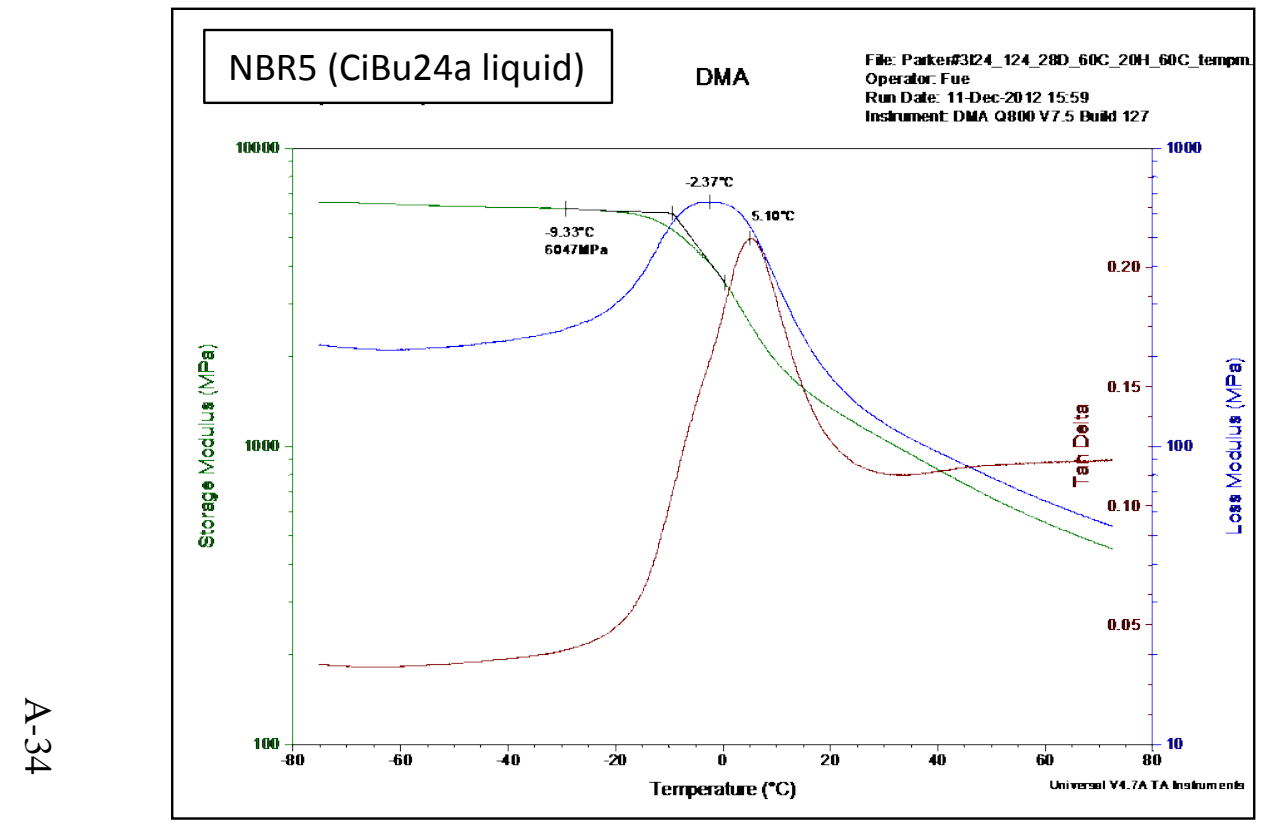

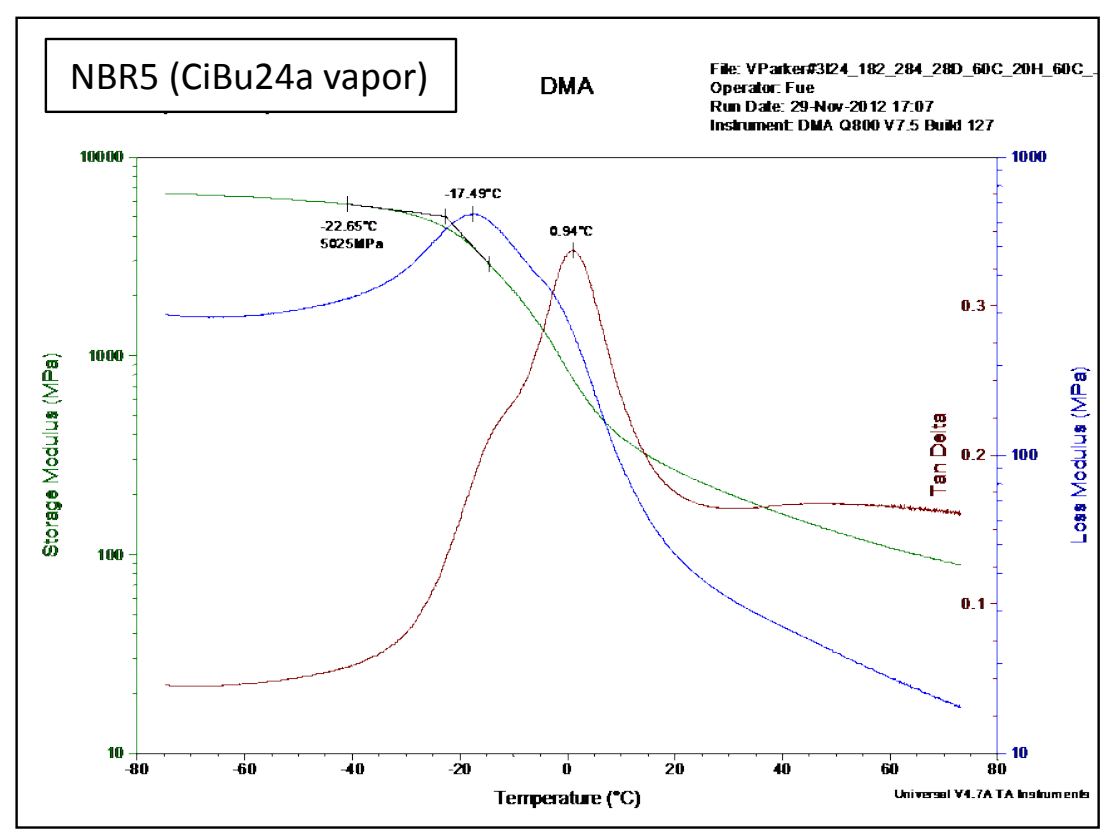


NBR\#6 DMA Results for Control and Fuel C Exposures
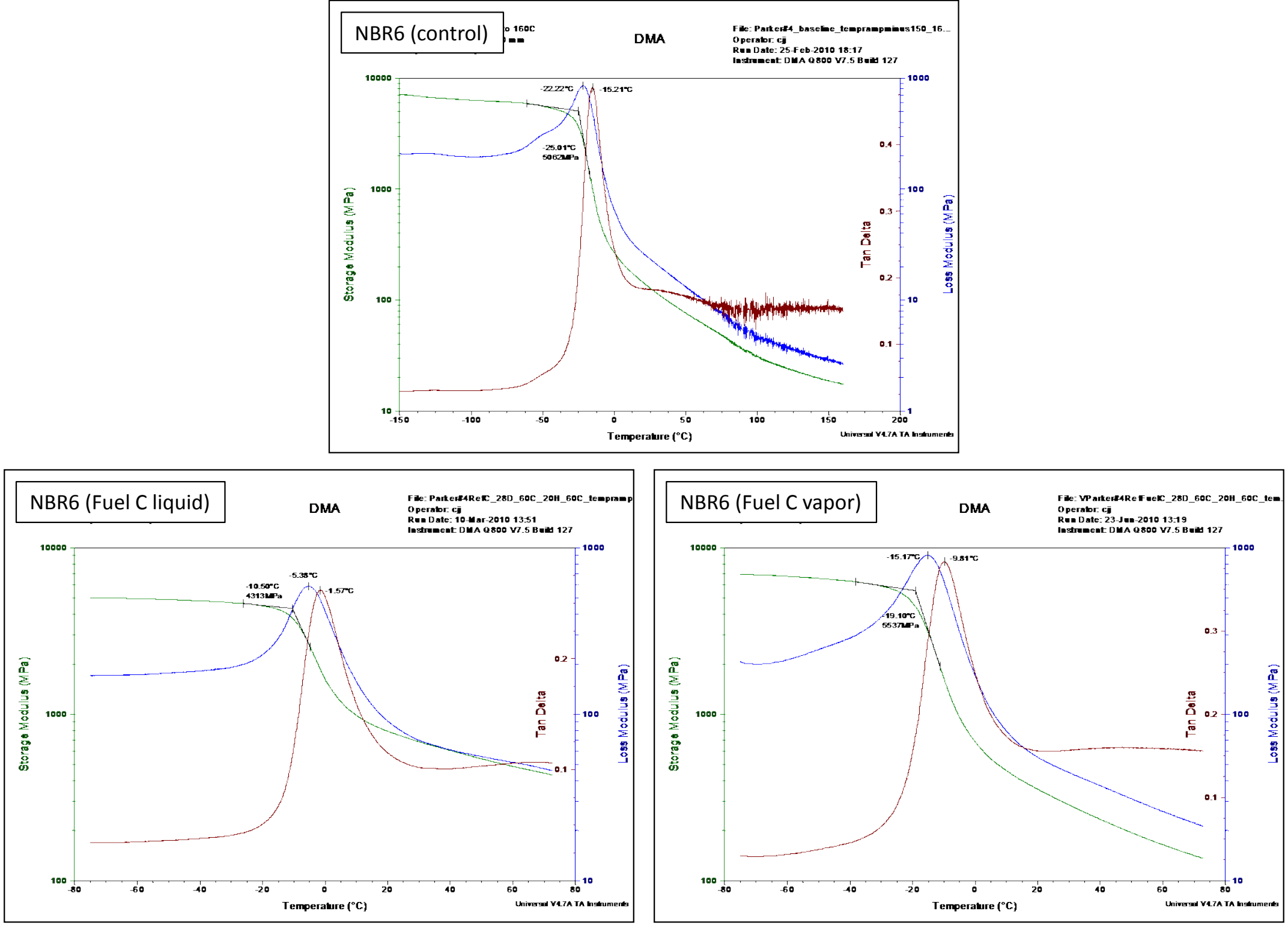


\section{NBR\#6 DMA Results for CE10a and CE17a Exposures}
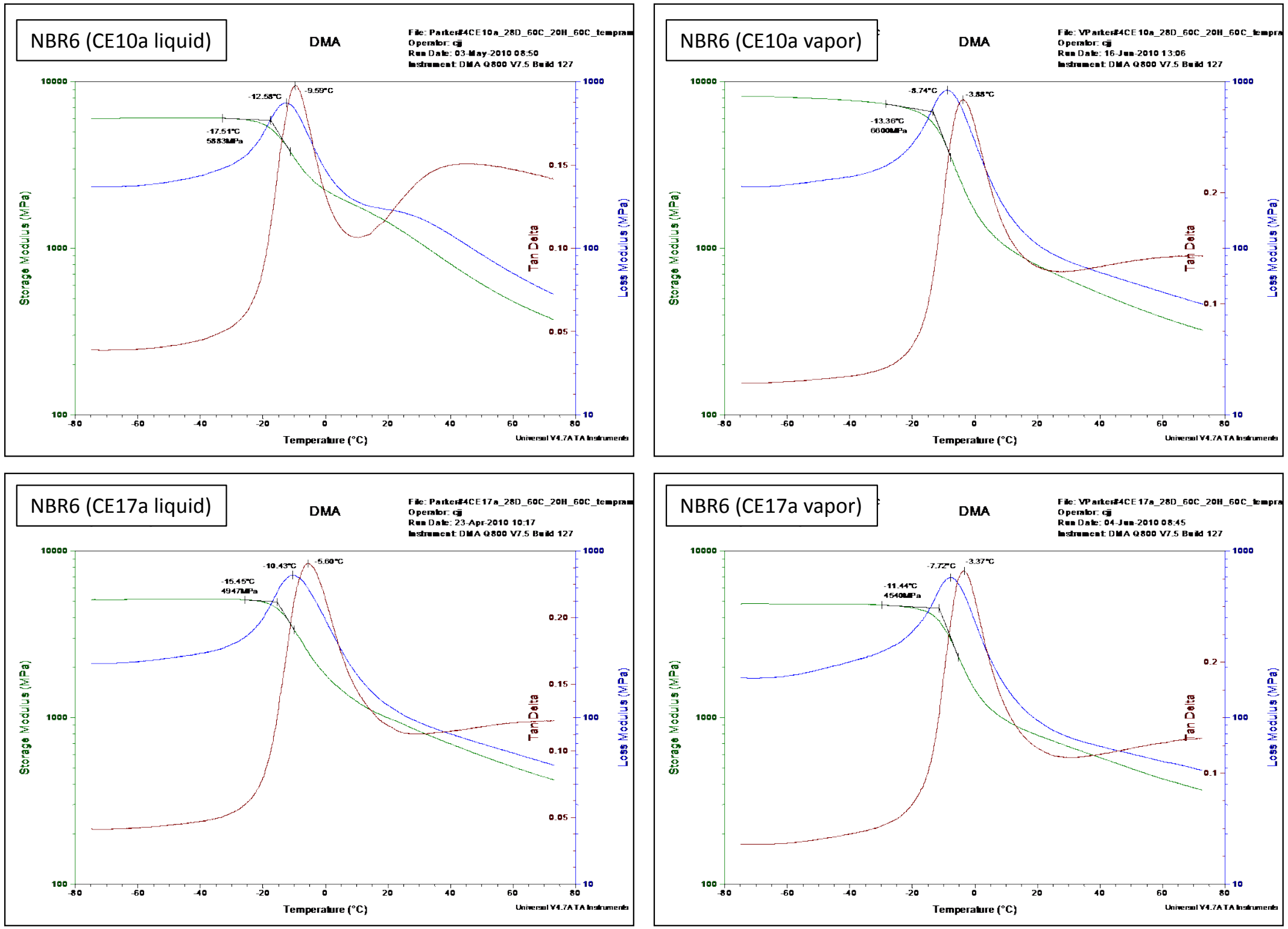
NBR\#6 DMA Results for CE25a and CiBu16a Exposures

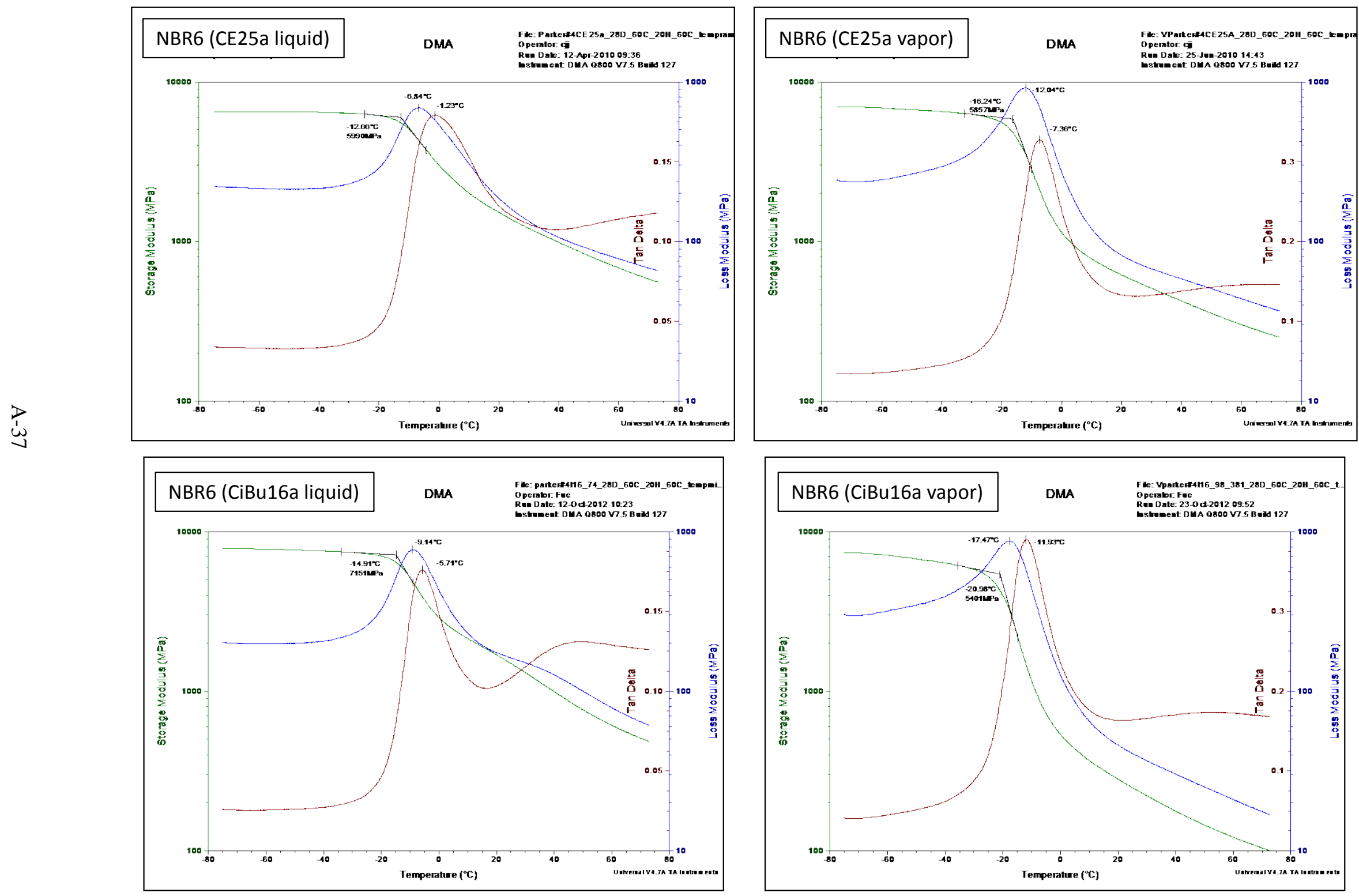




\section{NBR\#6 DMA Results for CiBu24a Exposures}
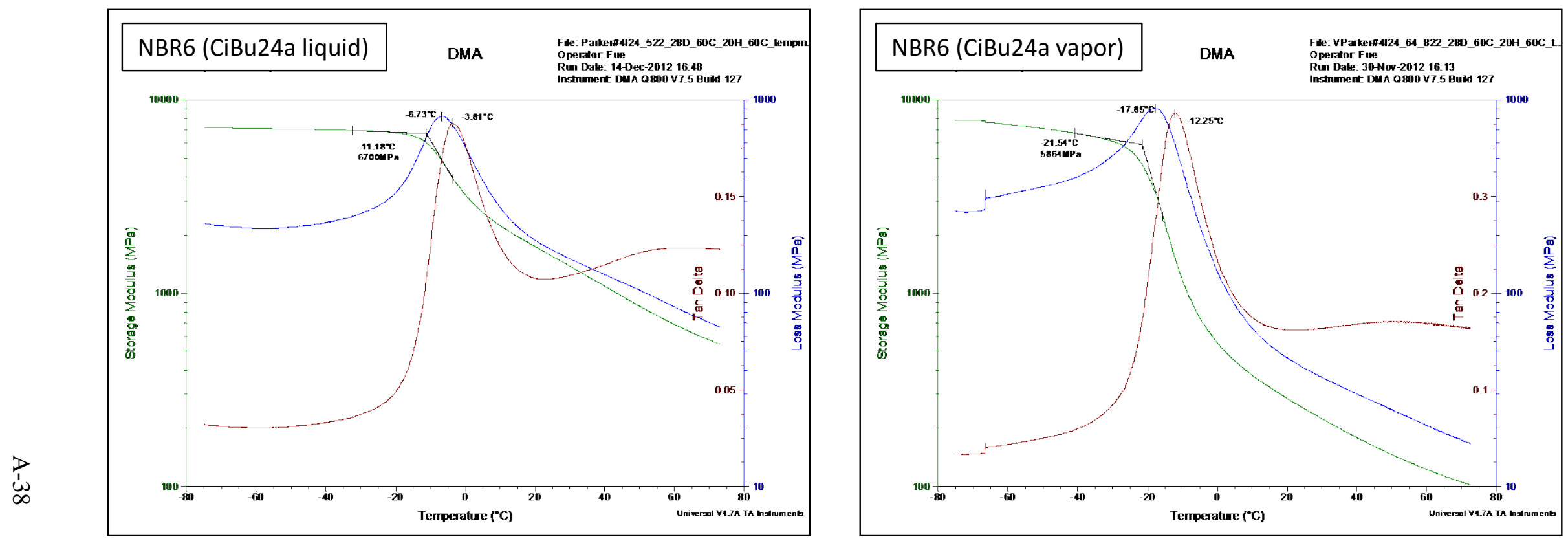


\section{Polyurethane DMA Results for Control and Fuel C Exposures}
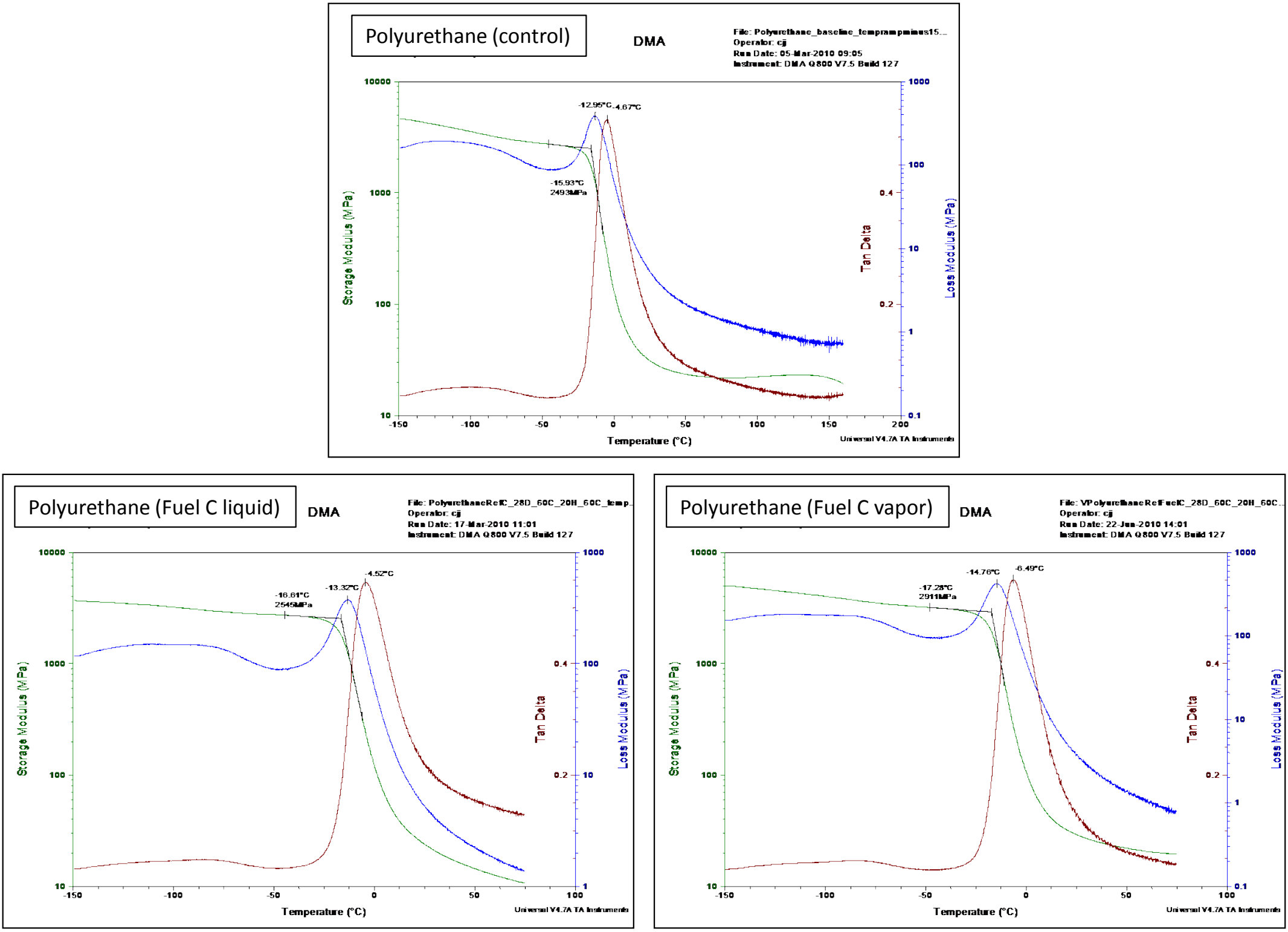
Polyurethane DMA Results for CE10a and CE17a Exposures
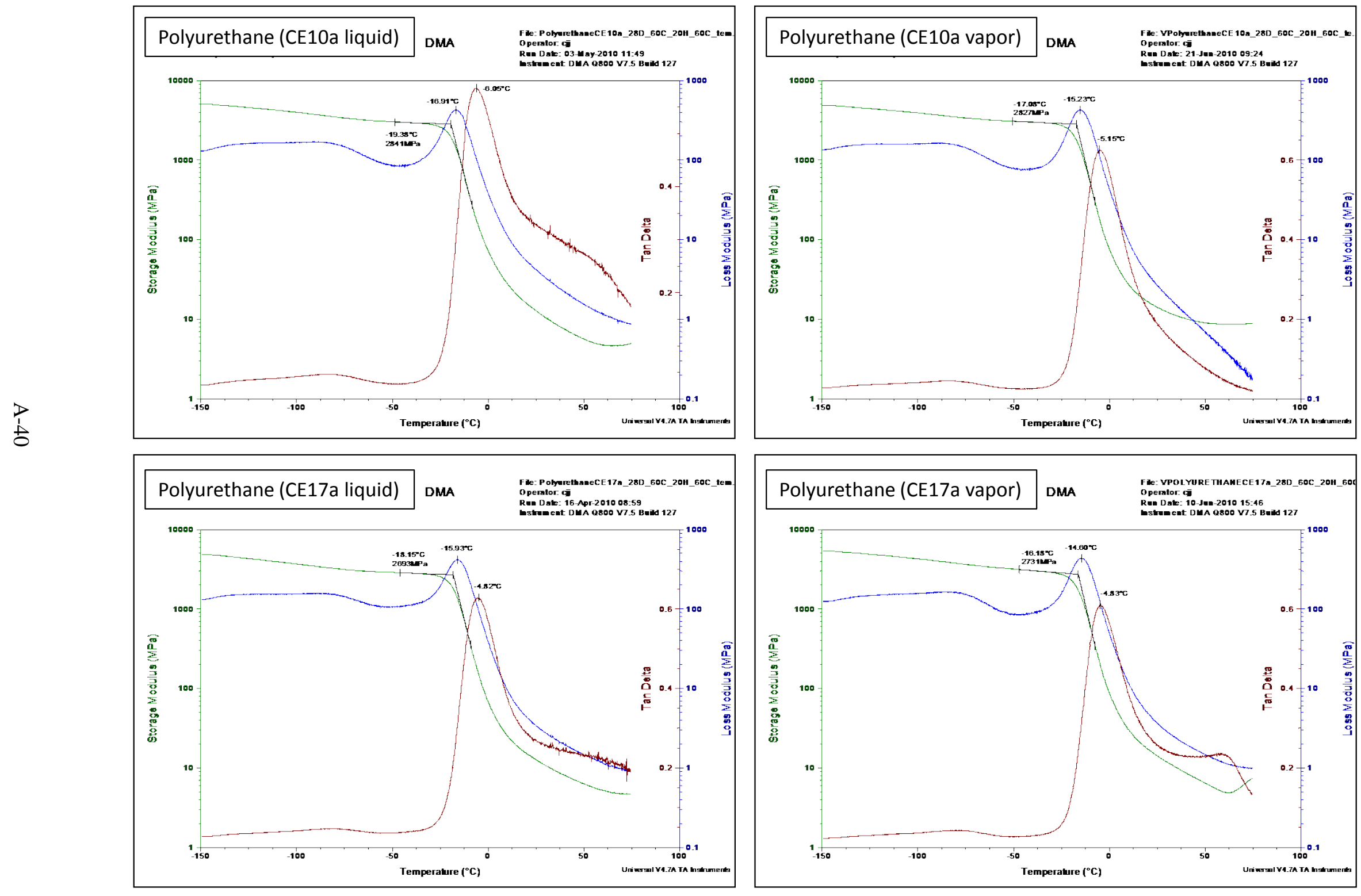
Polyurethane DMA Results for CE25a and CiBu16a Exposures
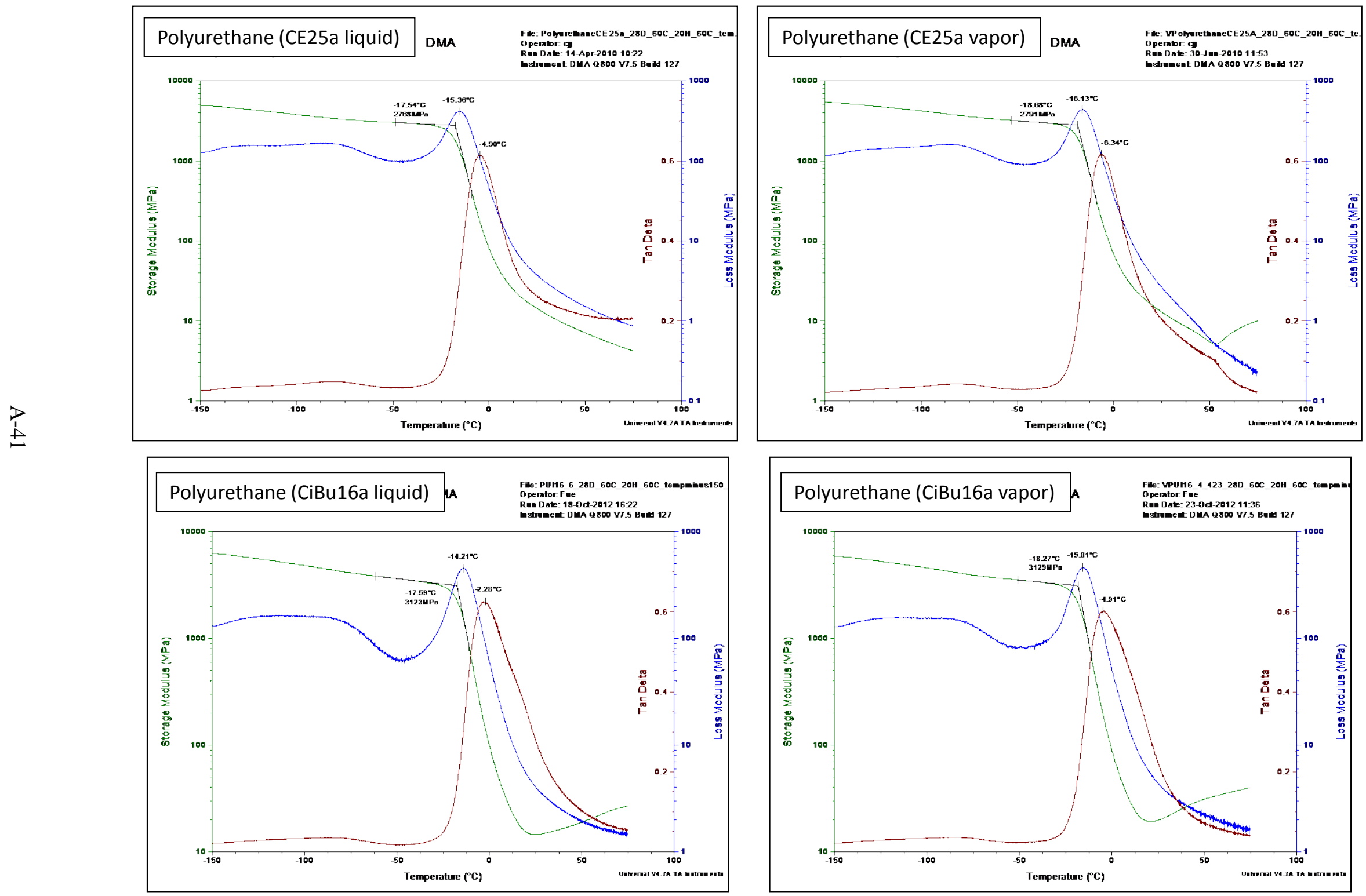


\section{Polyurethane DMA Results for CiBu24a Exposures}
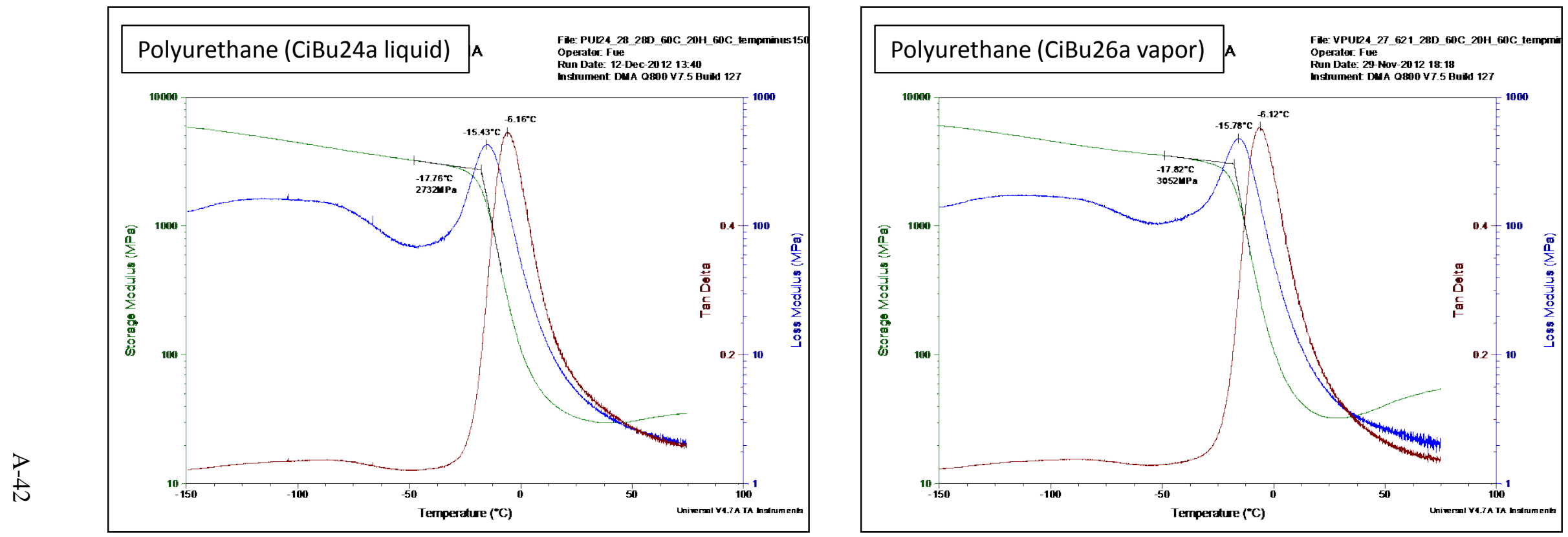


\section{Neoprene DMA Results for Control and Fuel C Exposures}
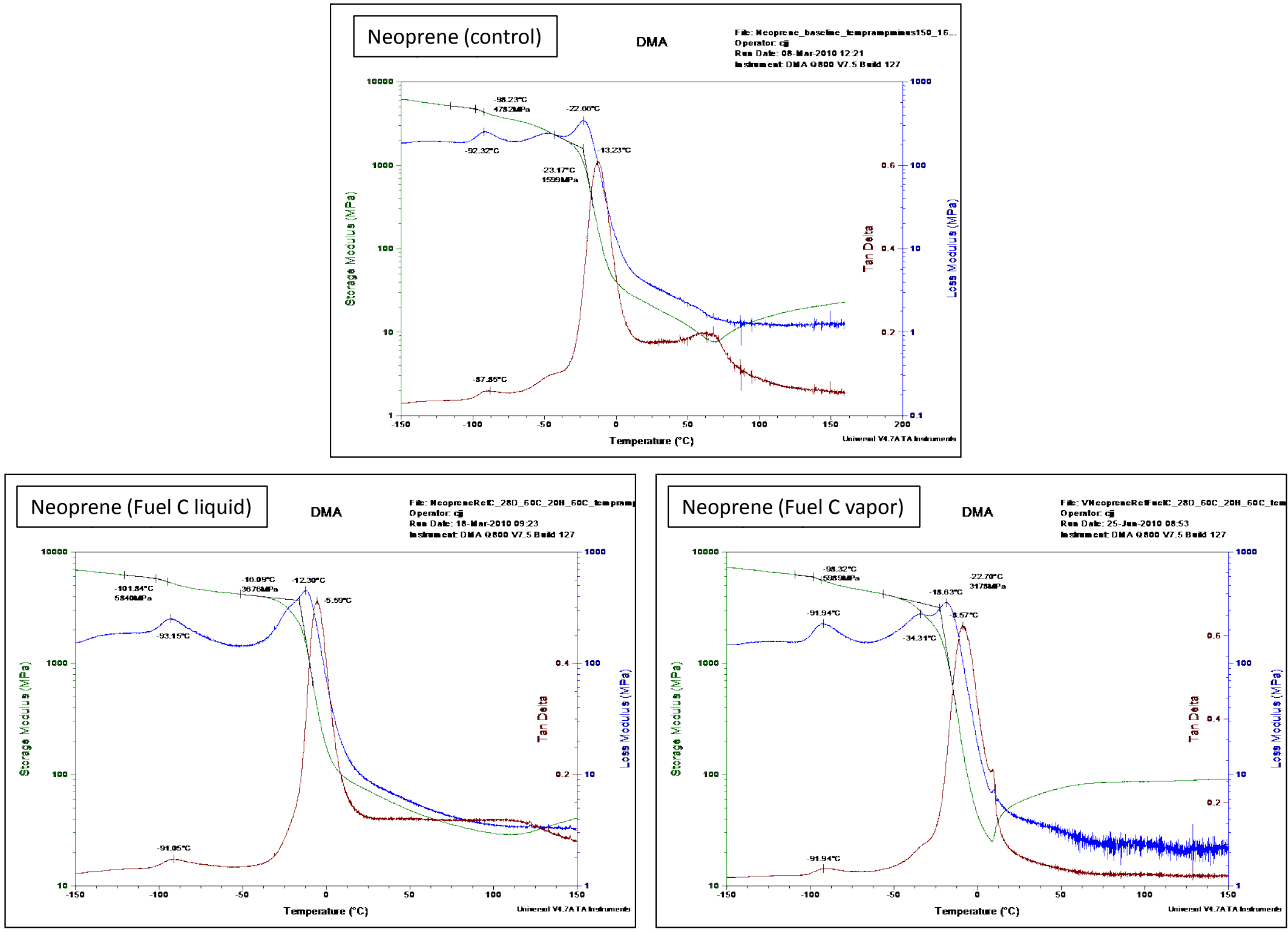


\section{Neoprene DMA Results for CE10a and CE17a}
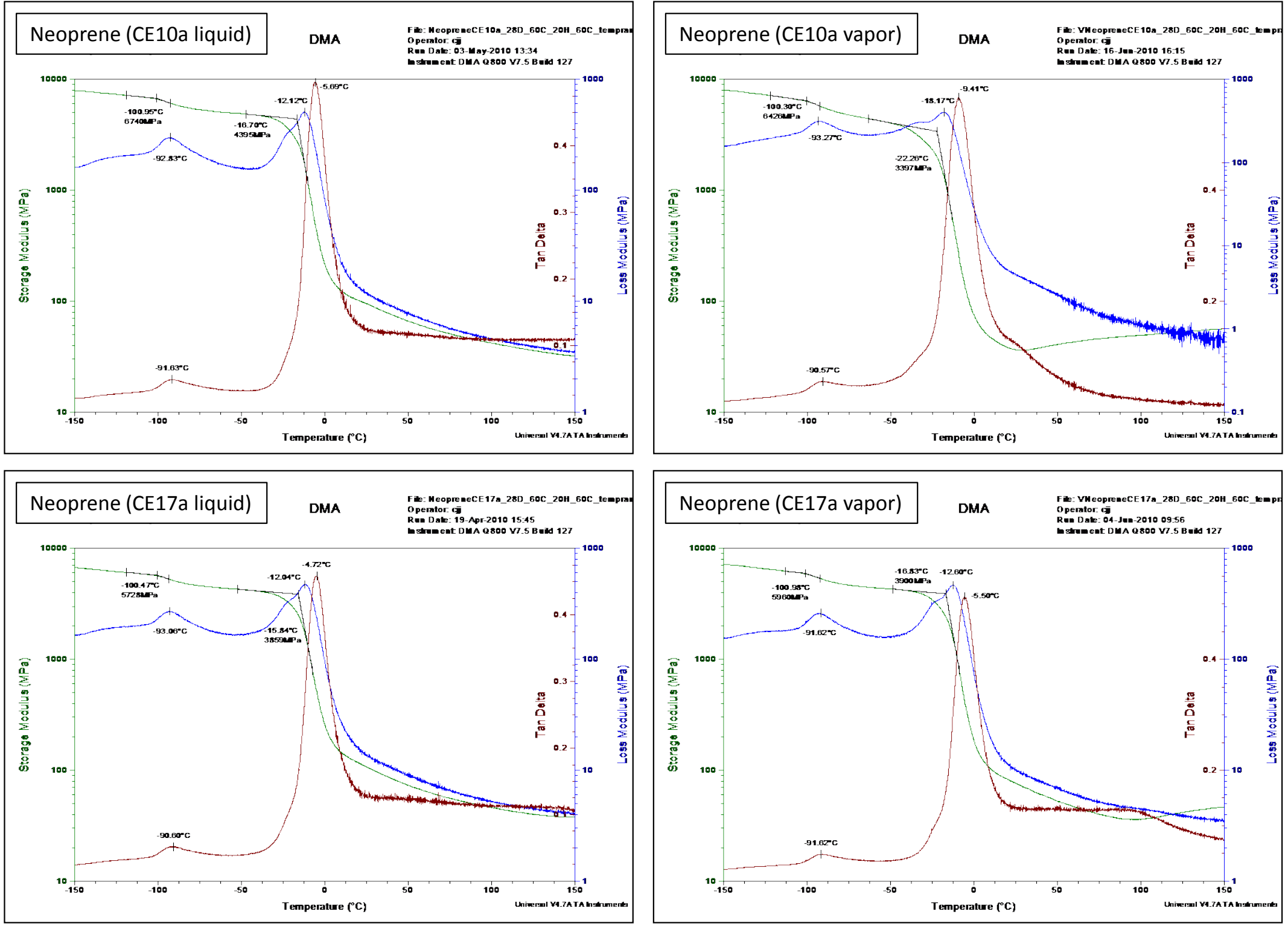


\section{Neoprene DMA Results for CE25a and CiBu16a}
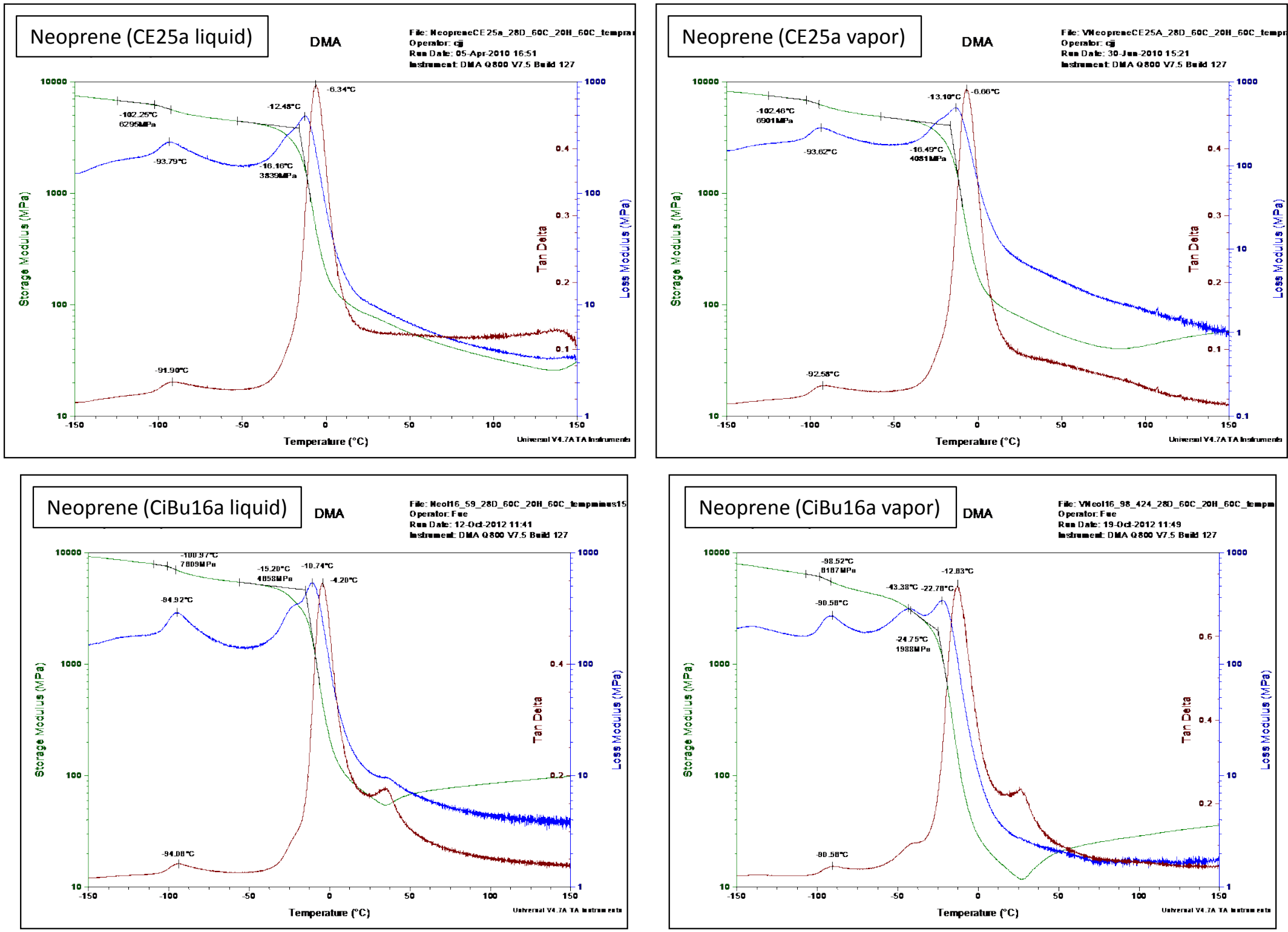


\section{Neoprene DMA Results for CiBu24a}
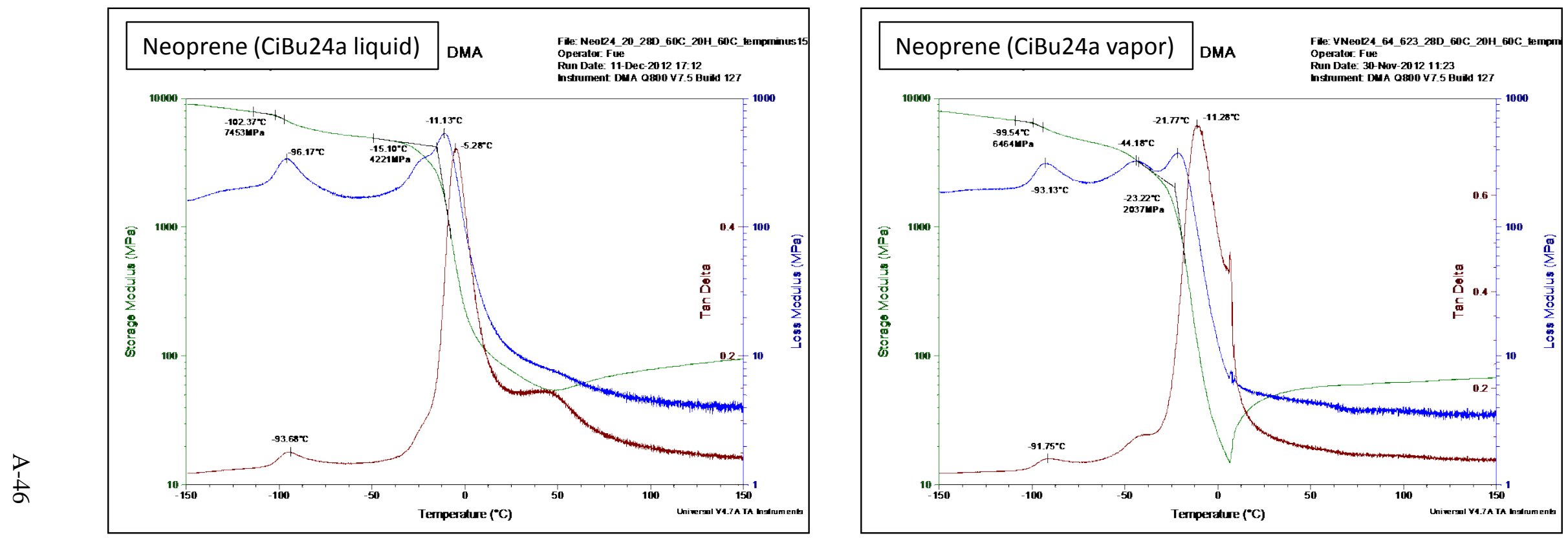
SBR DMA Results for Control and Fuel C Exposures
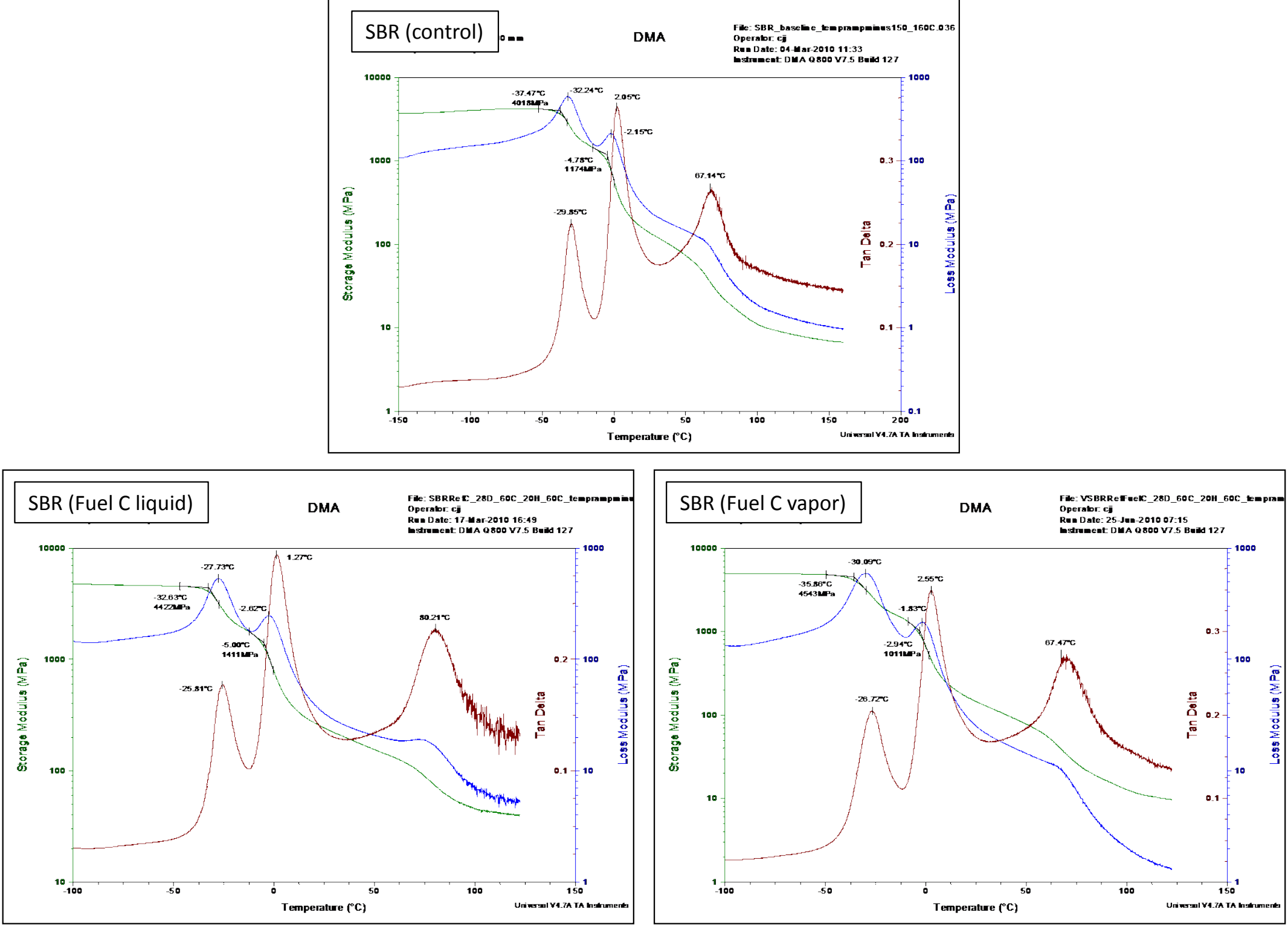
SBR DMA Results for CE10a and CE17a Exposures
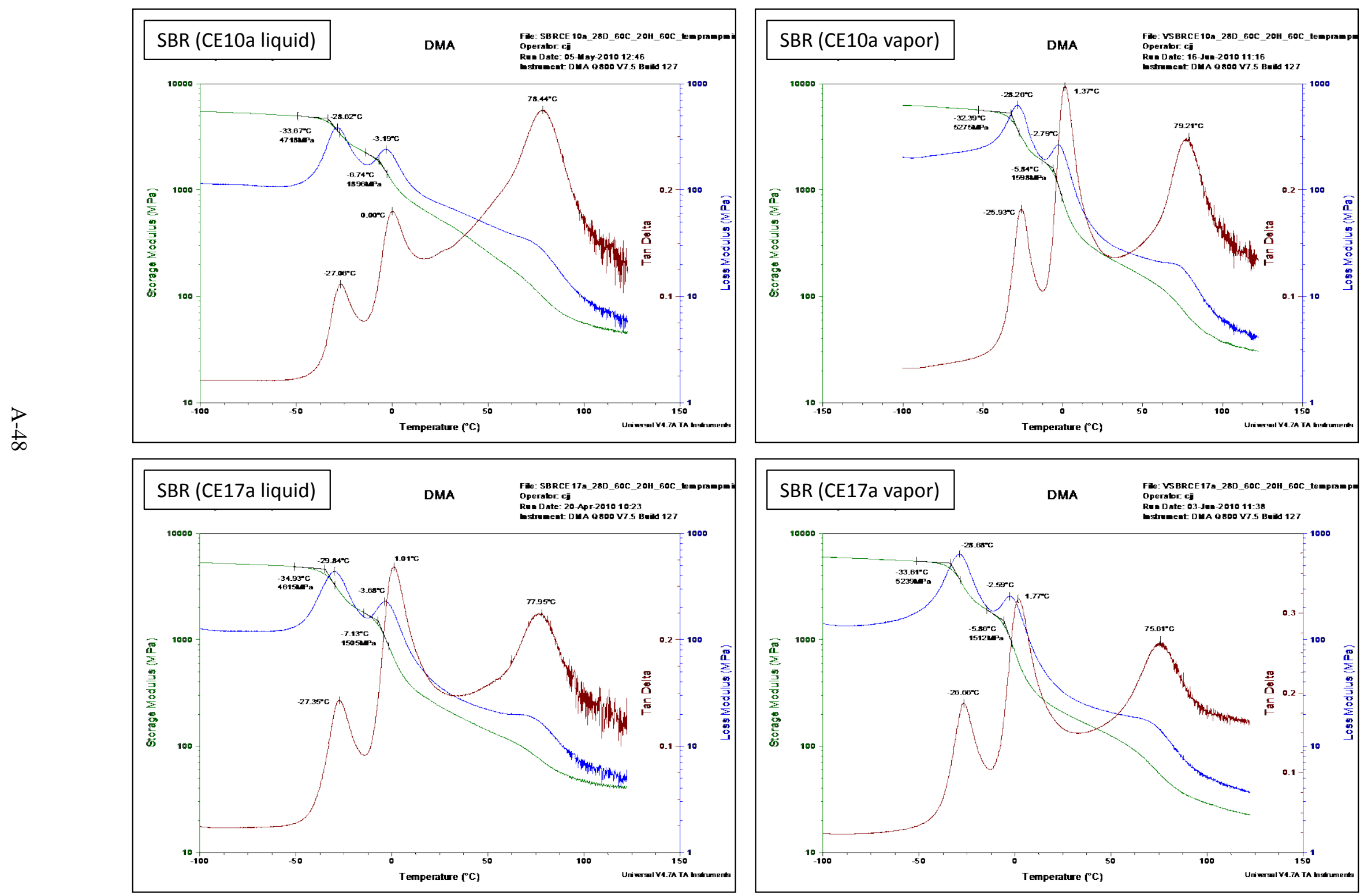
SBR DMA Results for CE25a and CiBu16a Exposures
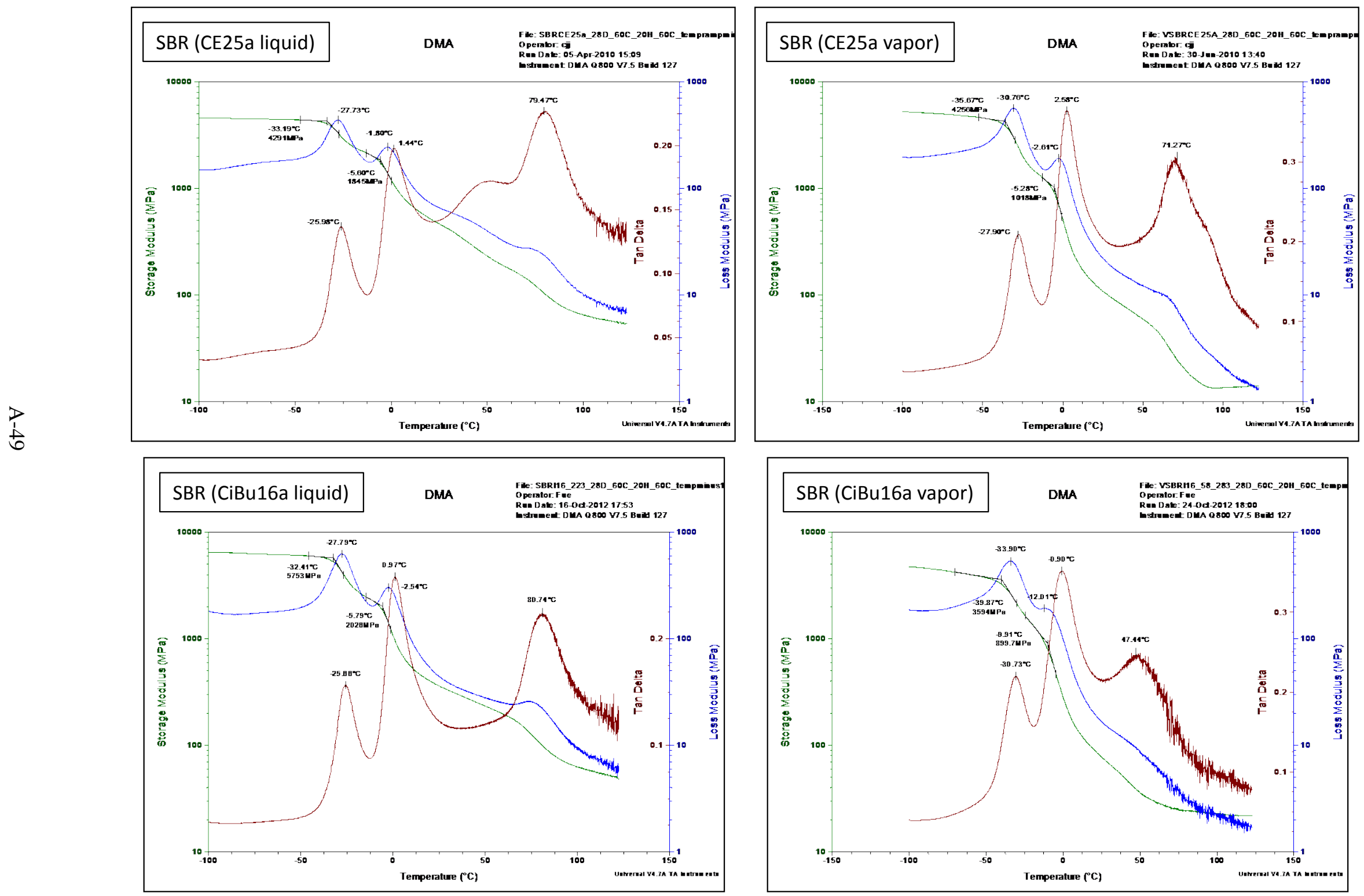
SBR DMA Results for CiBu24a Exposures

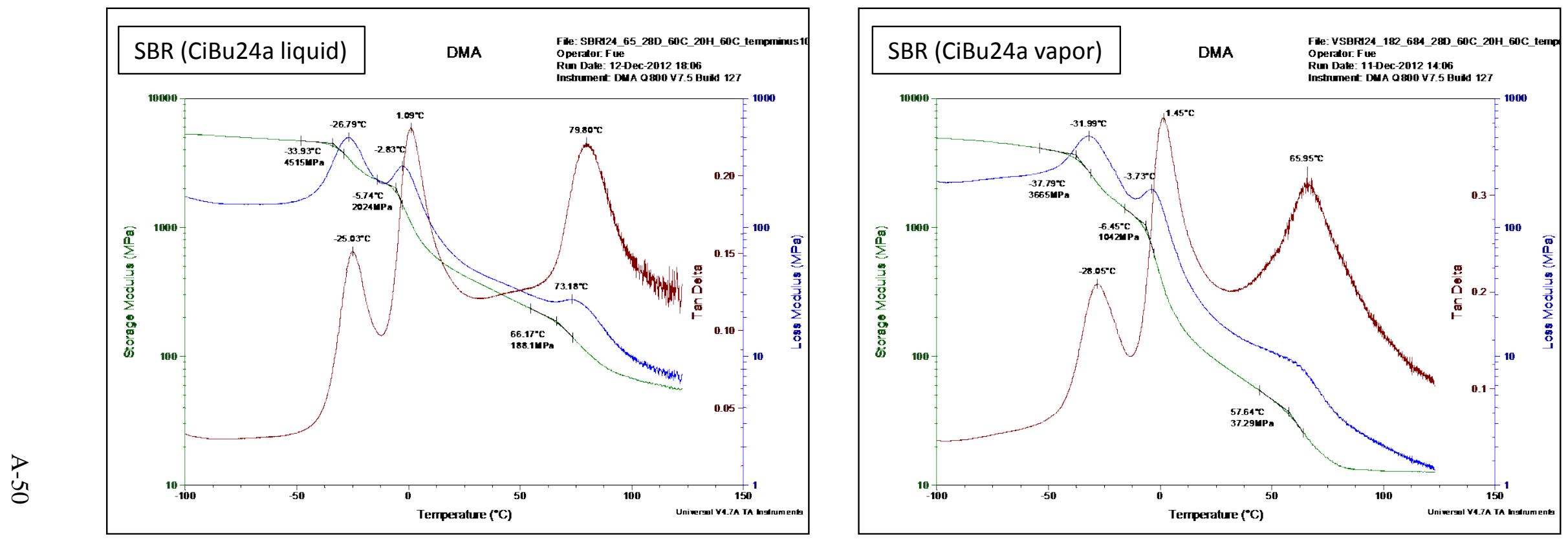




\section{Silicone DMA Results for Control and Fuel C Exposures}
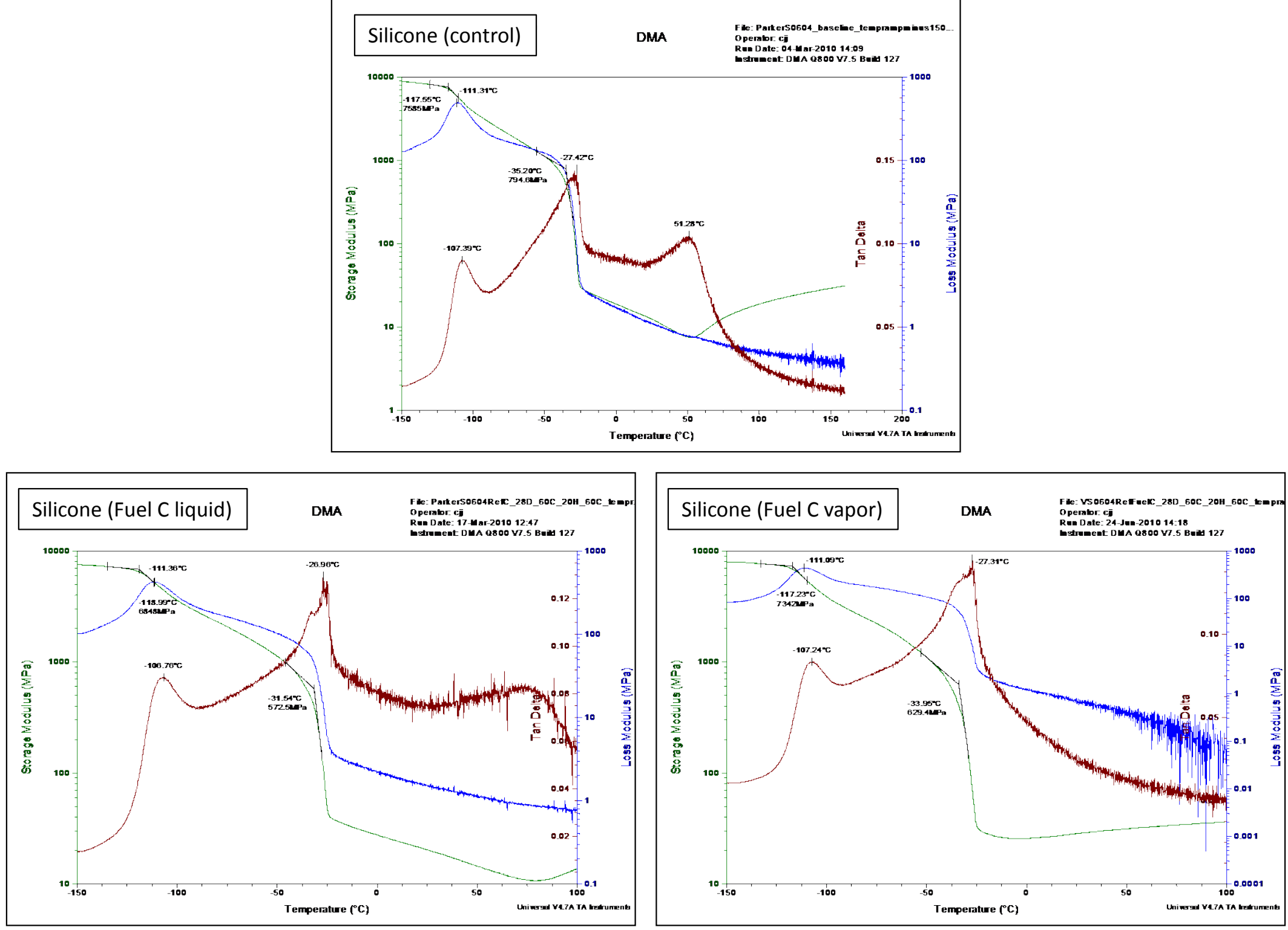
Silicone DMA Results for CE10a and CE17a Exposures
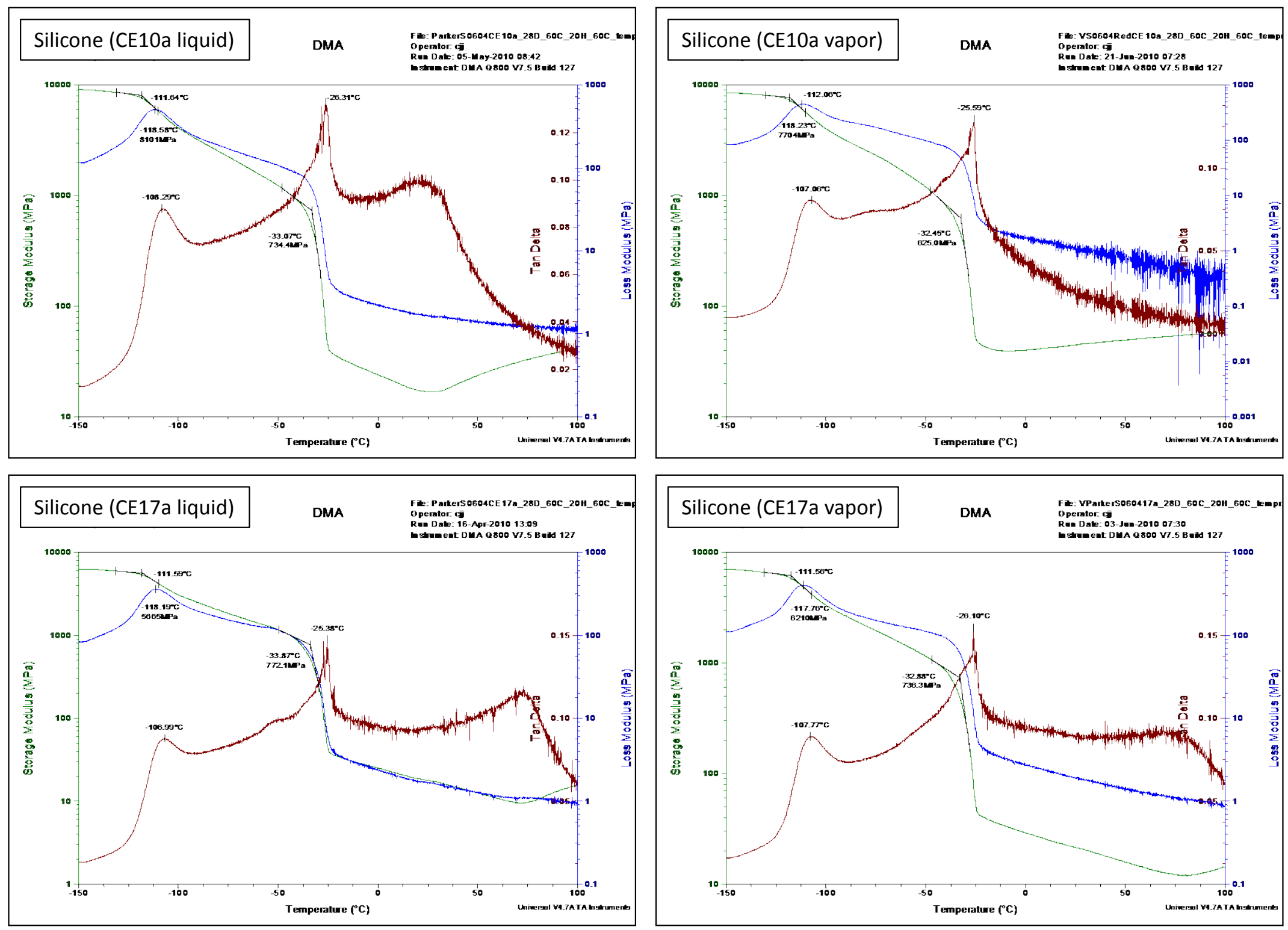


\section{Silicone DMA Results for CE25a and CiBu16a Exposures}
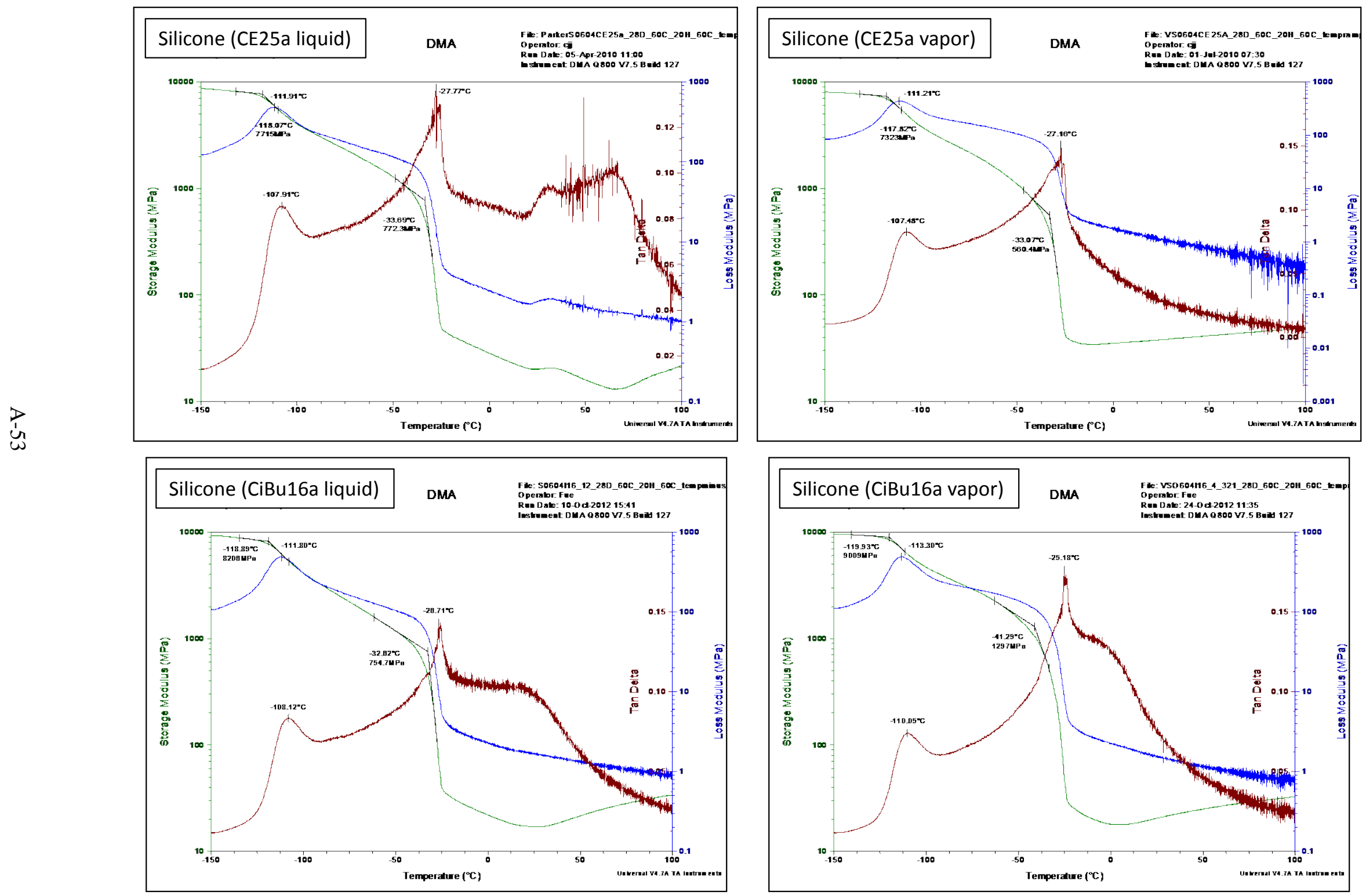


\section{Silicone DMA Results for CiBu24a Exposures}

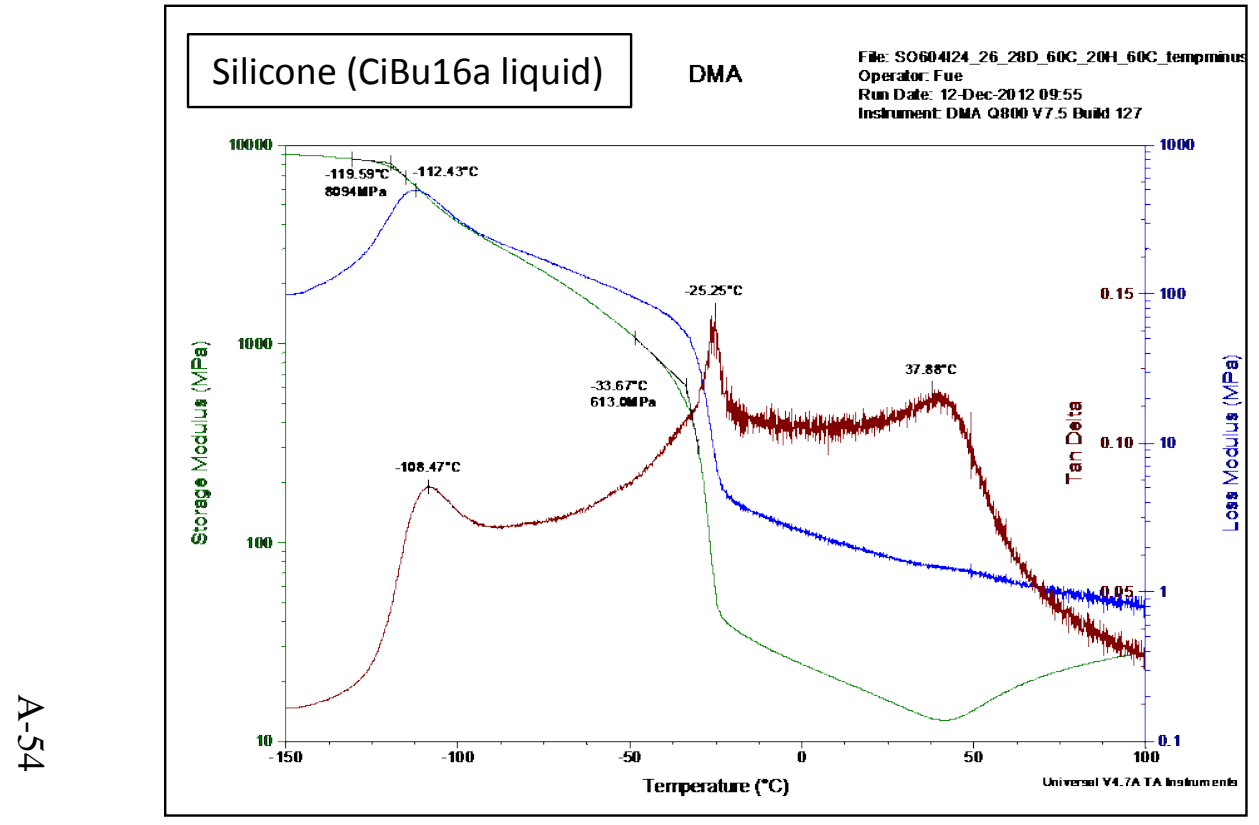

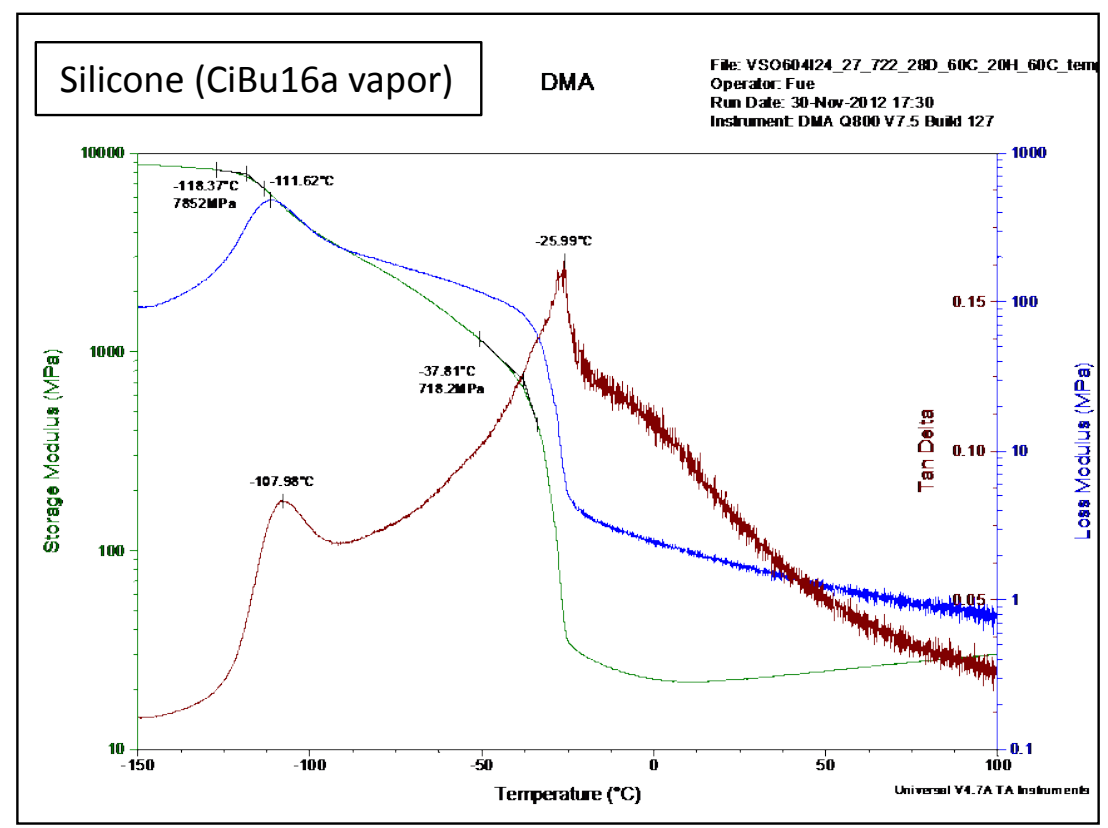


APPENDIX B. DMA RESULTS FOR PLASTICS AND CORK MATERIALS 

PPS DMA Results for Control and Fuel C Exposures
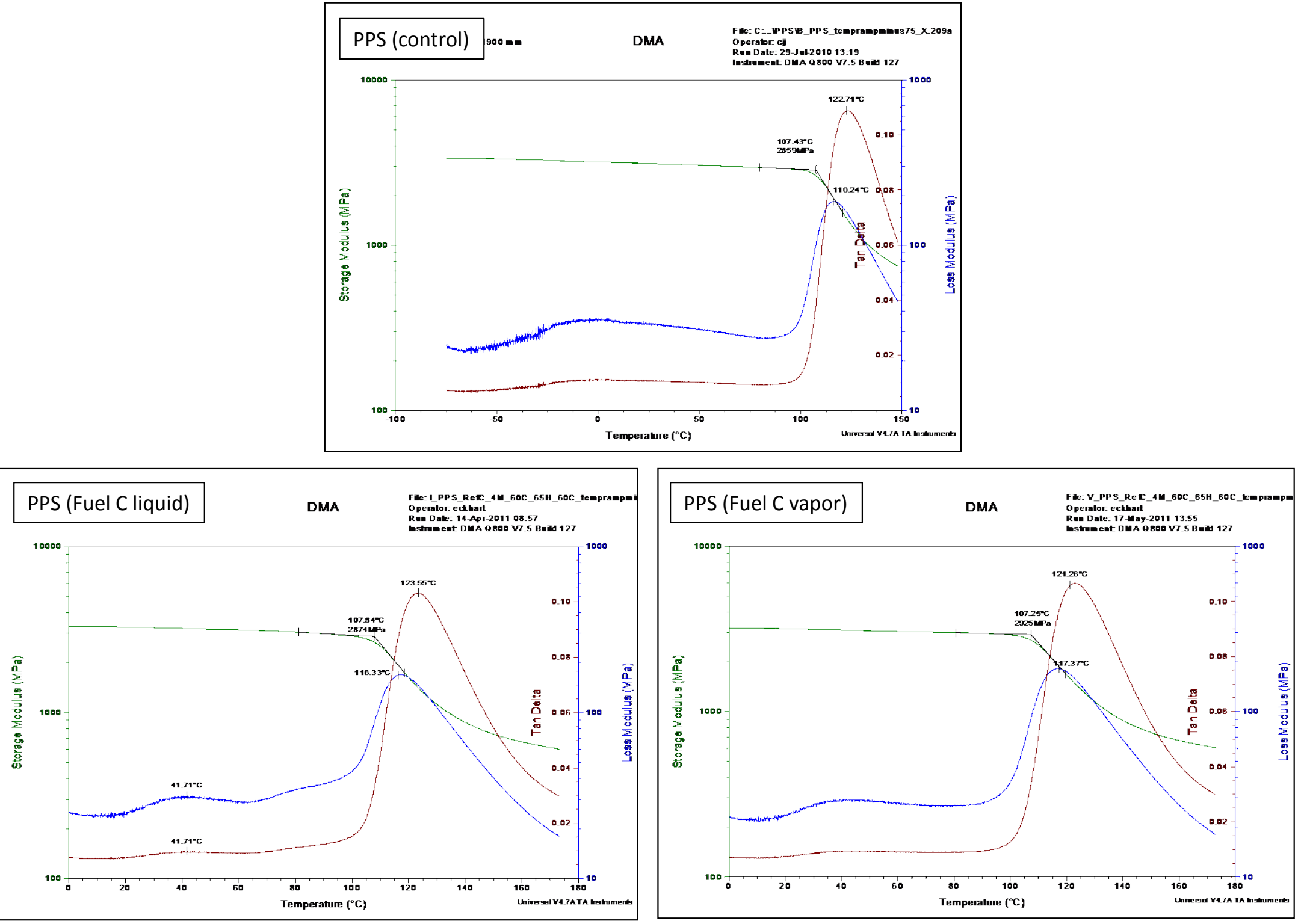


\section{PPS DMA Results for CE25a and CiBu16a Exposures}
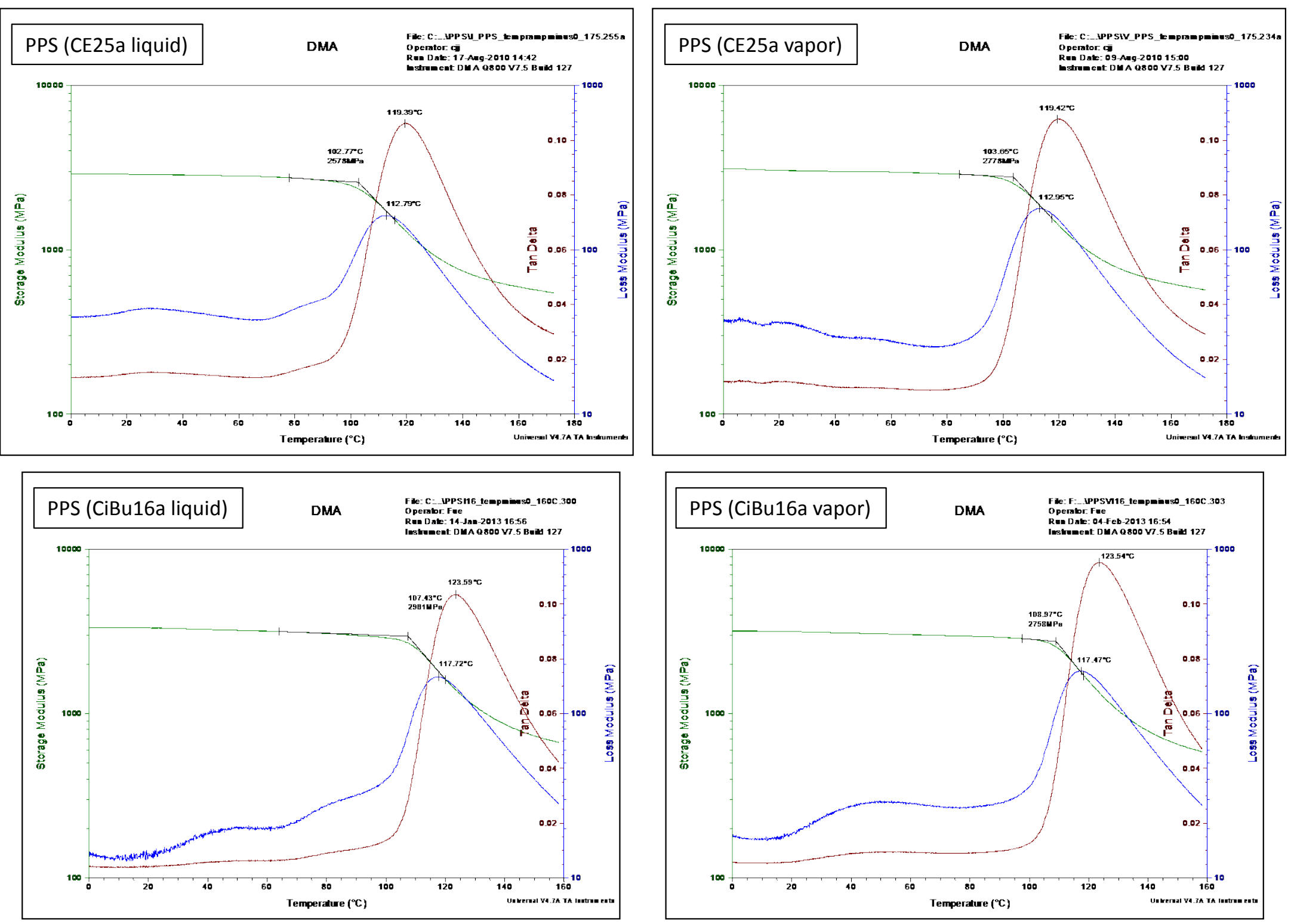
PPS DMA Results for CiBu24a Exposures
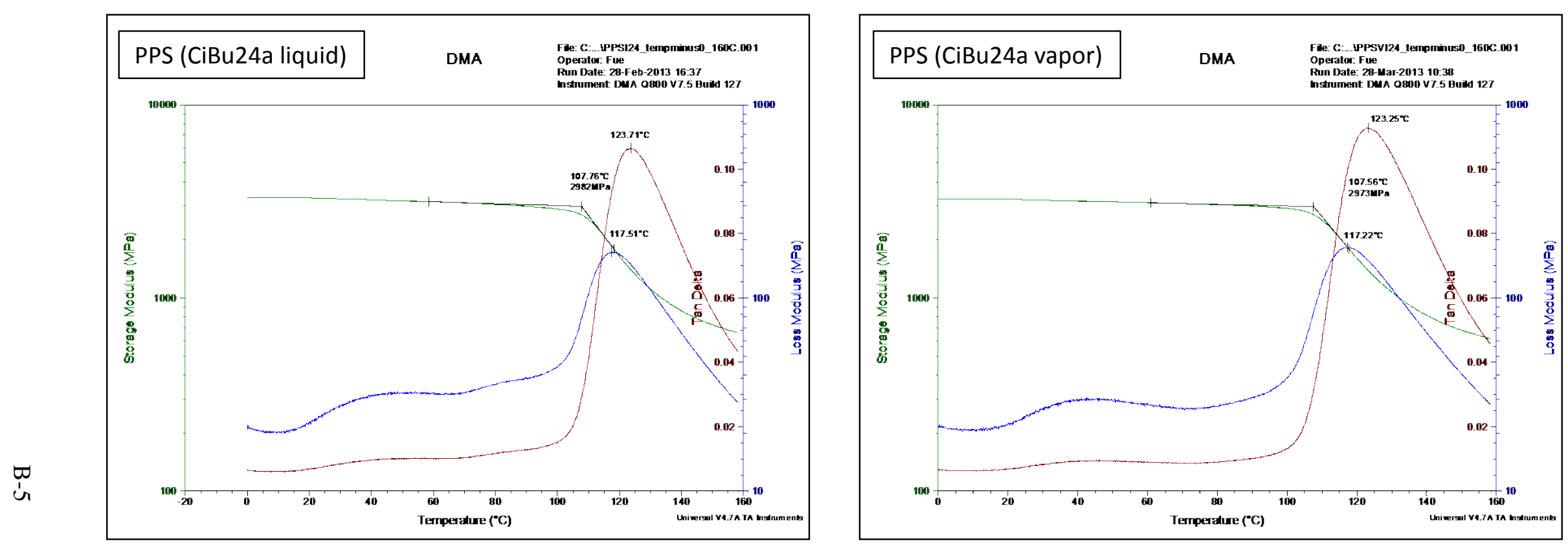


\section{PET DMA Results for Control and Fuel C Exposures}
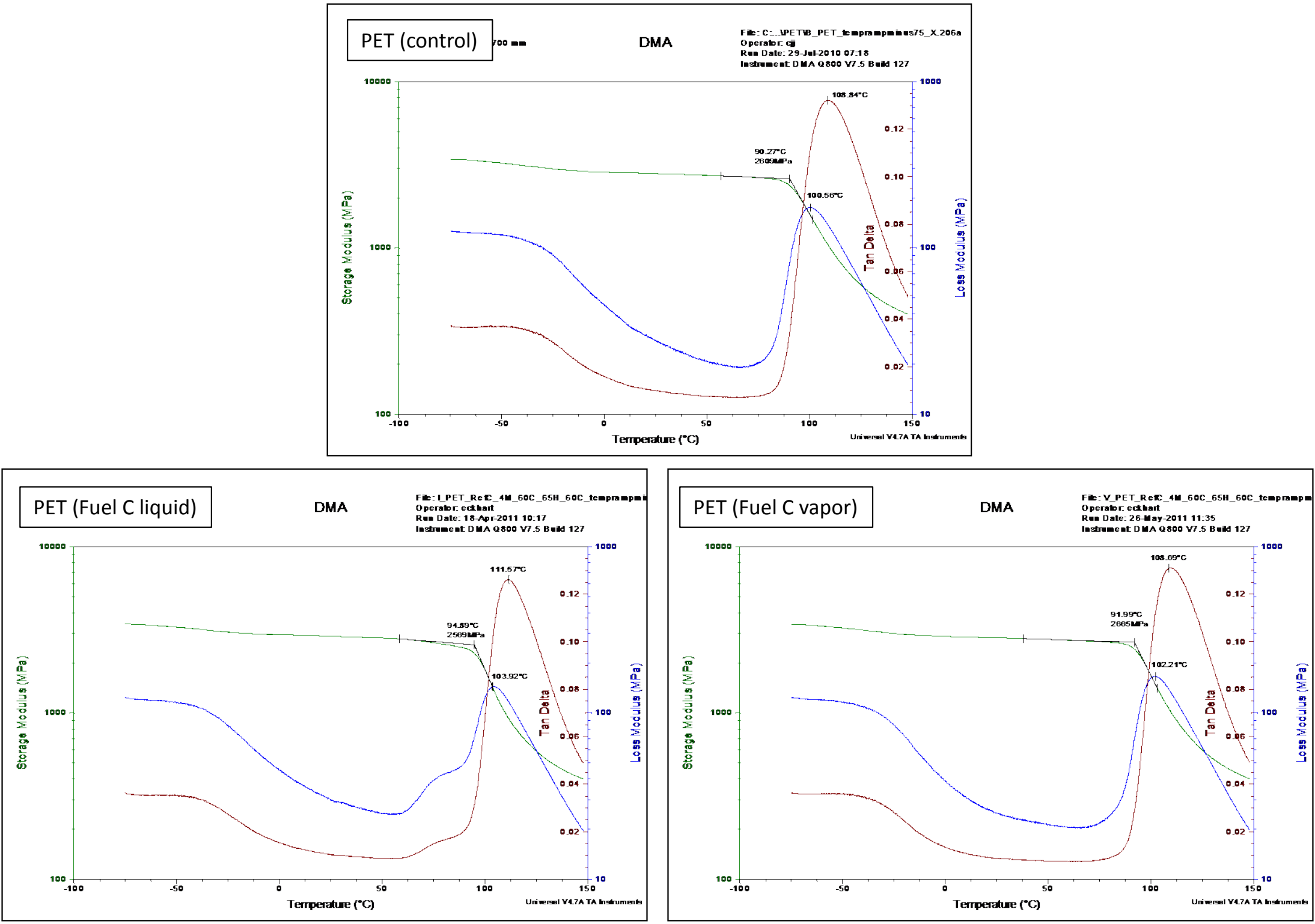
PET DMA Results for CE25a and CiBu16a Exposures
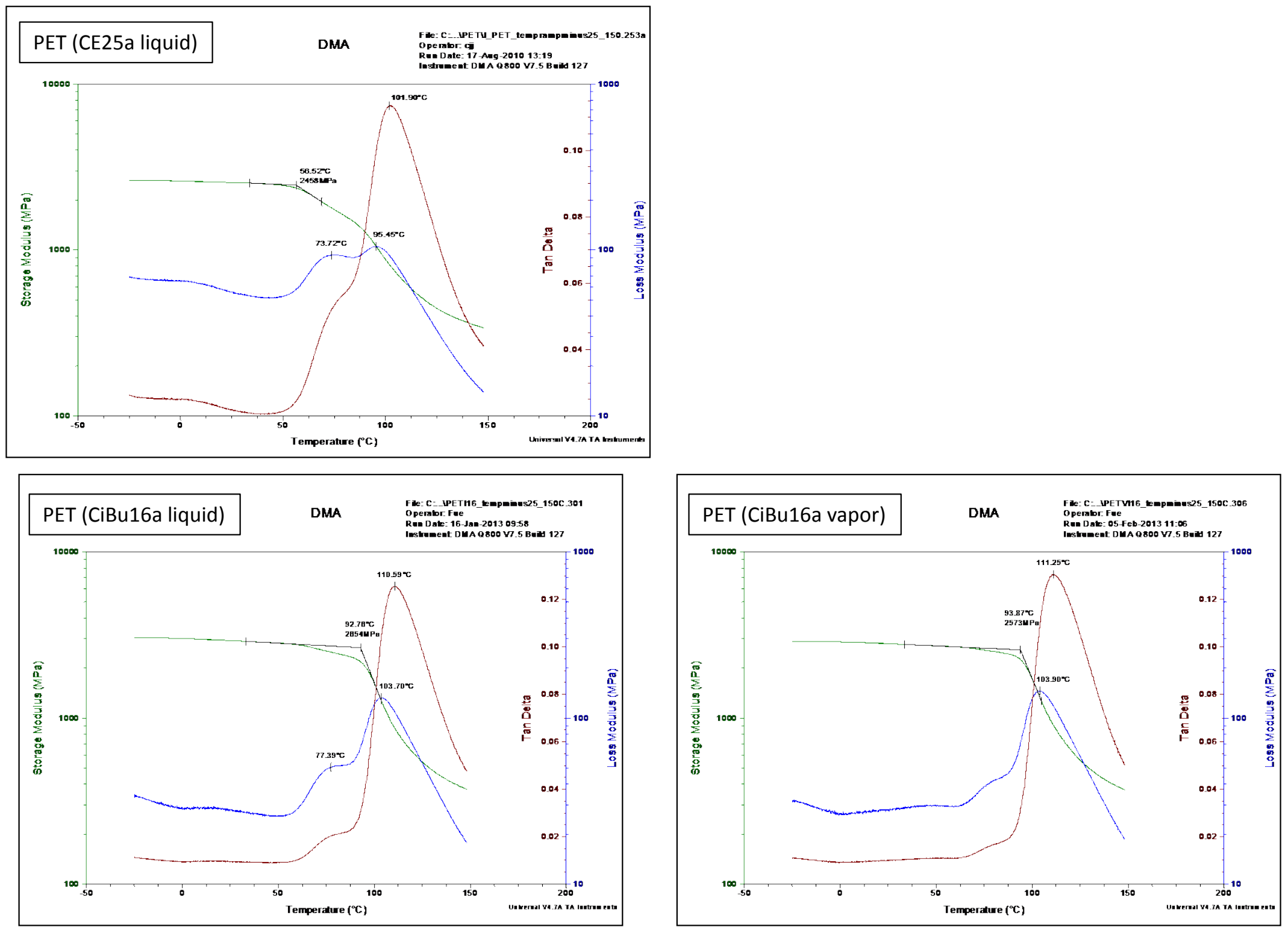
PET DMA Results for CiBu24a Exposures
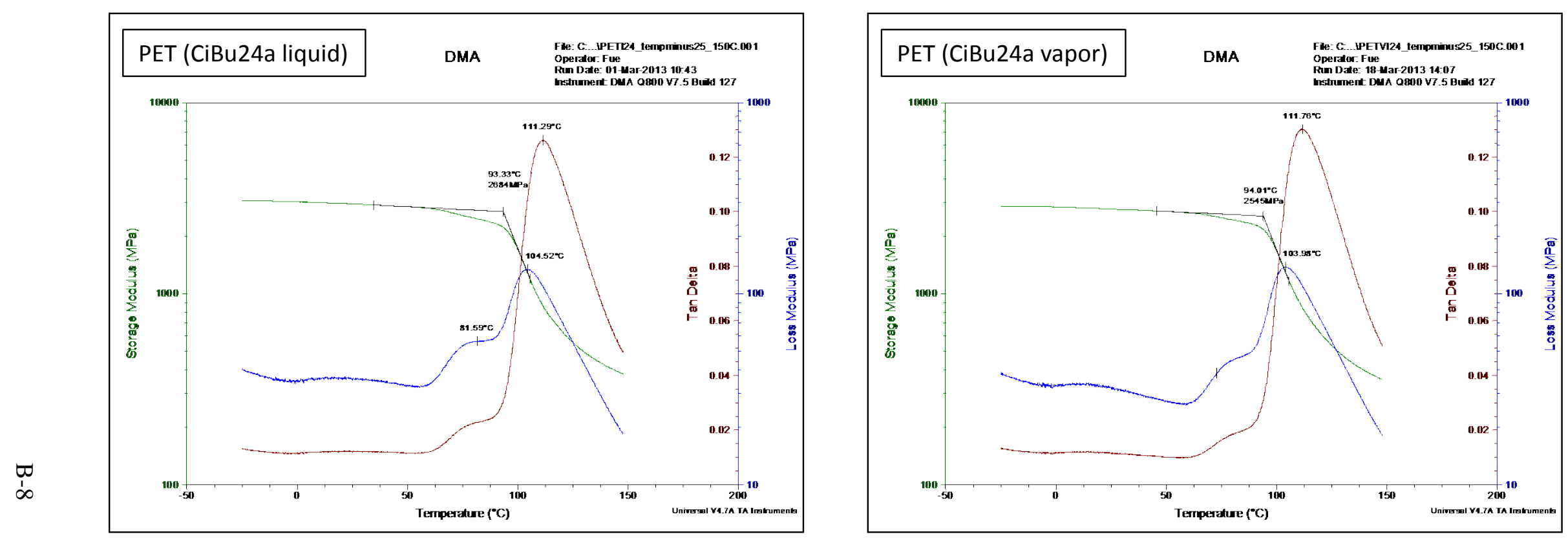
PTFE DMA Results for Control and Fuel C Exposures
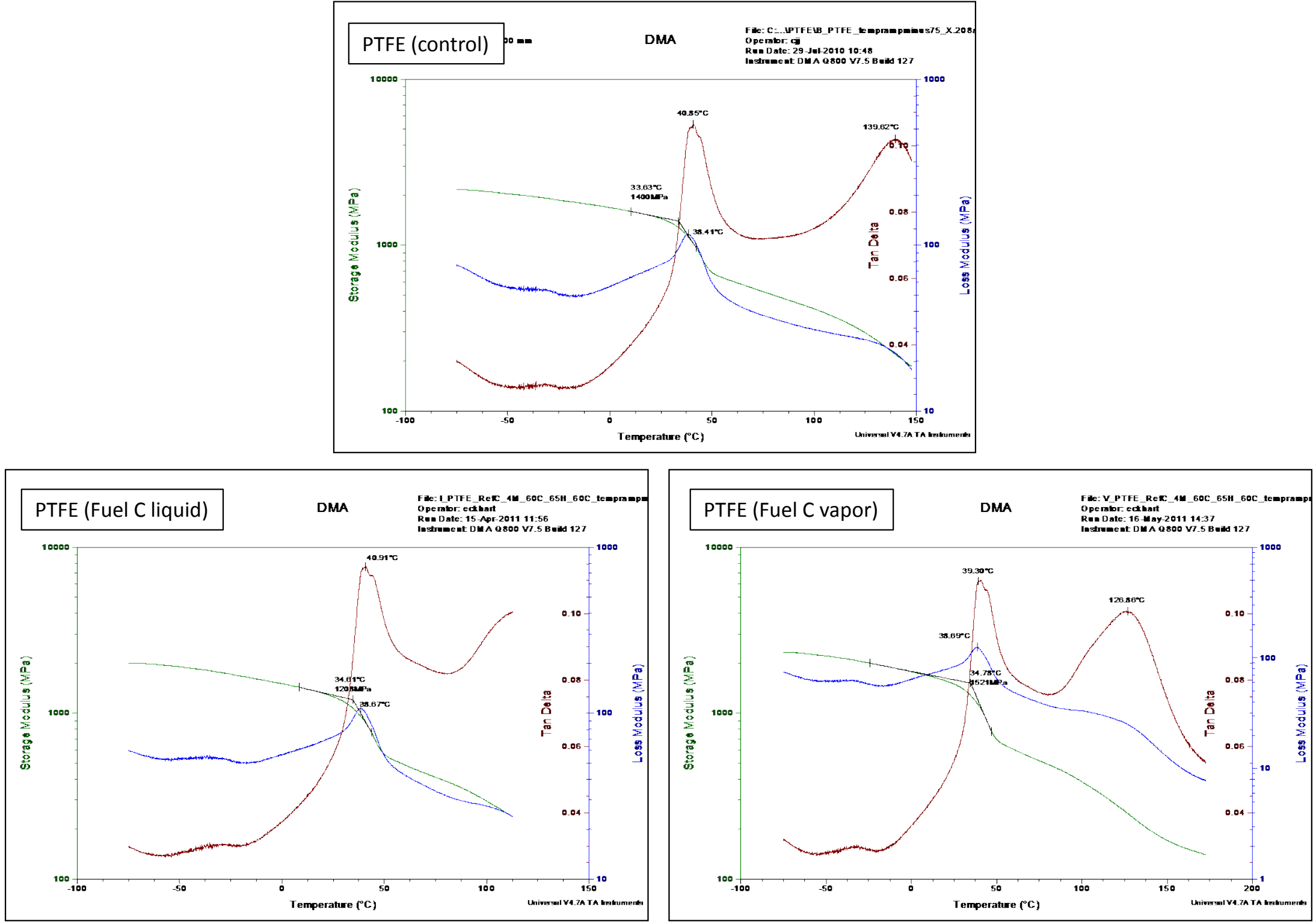


\section{PTFE DMA Results for CE25a and CiBu16a Exposures}
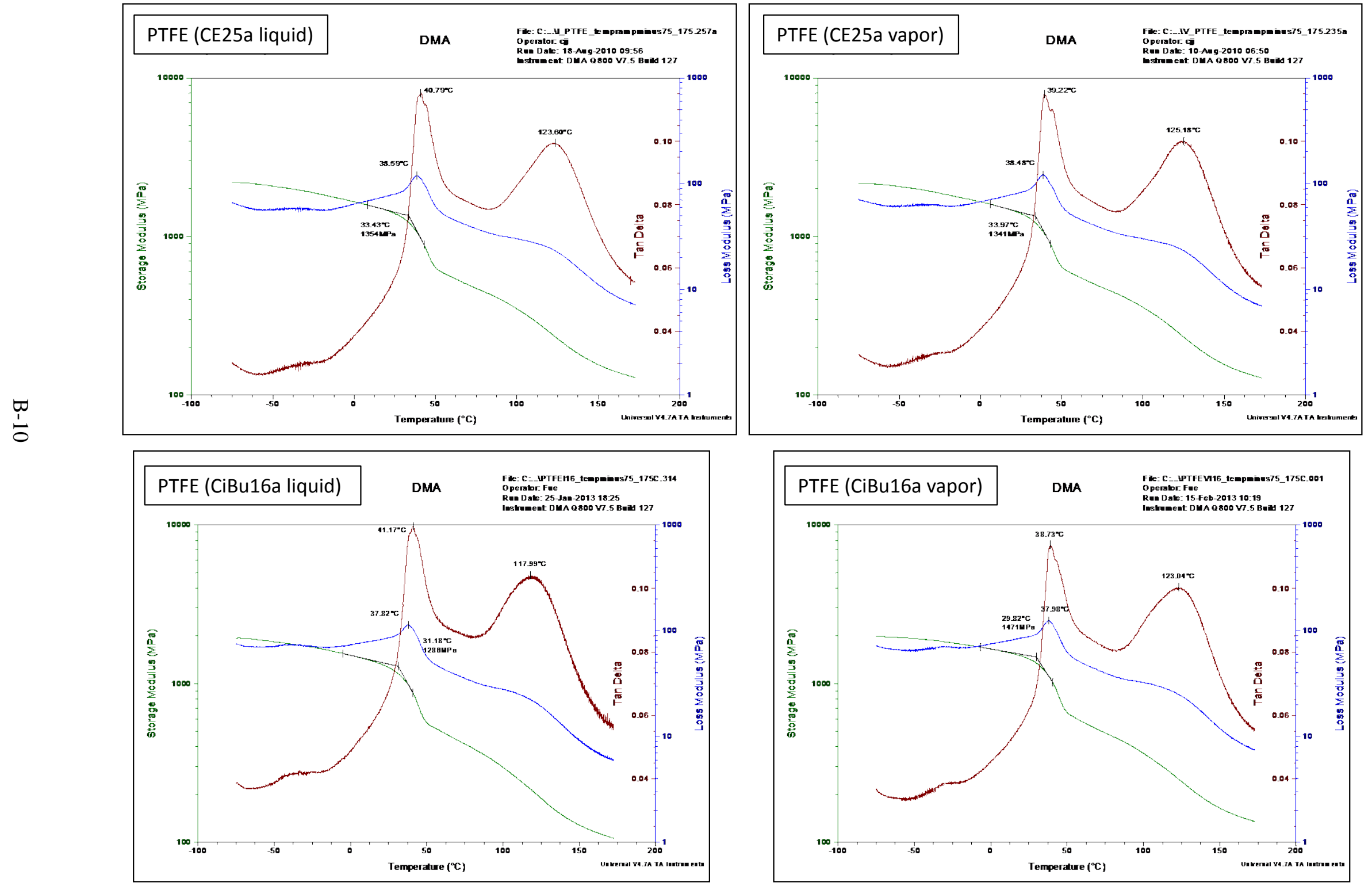
PTFE DMA Results for CiBu24a Exposures
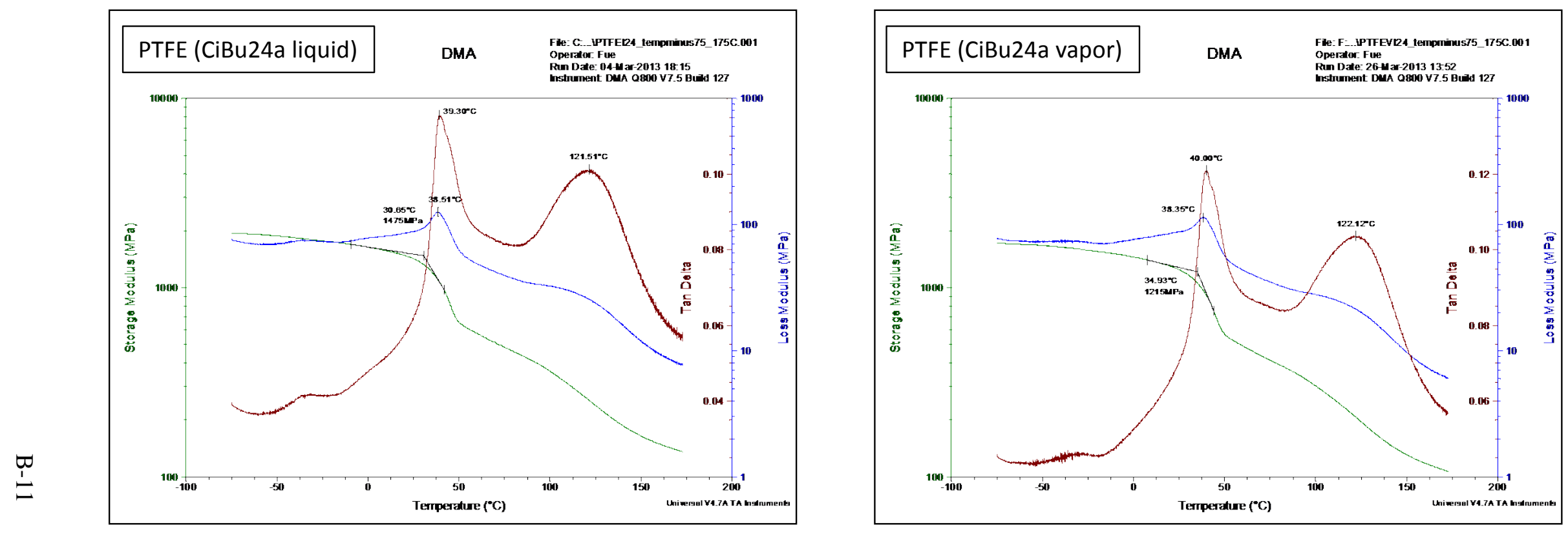


\section{PVDF DMA Results for Control and Fuel C Exposures}
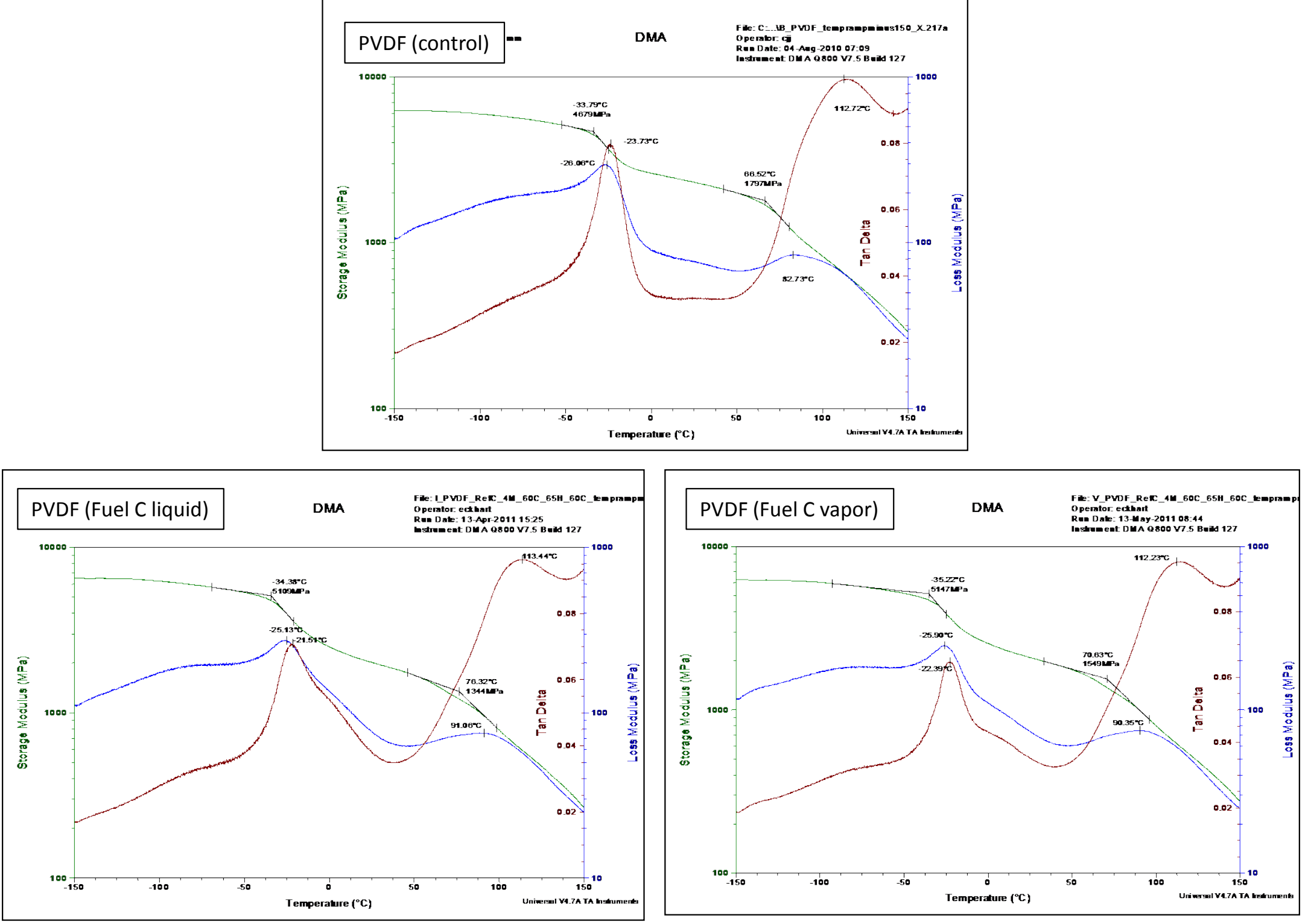
PVDF DMA Results for CE25a and CiBu16a Exposures
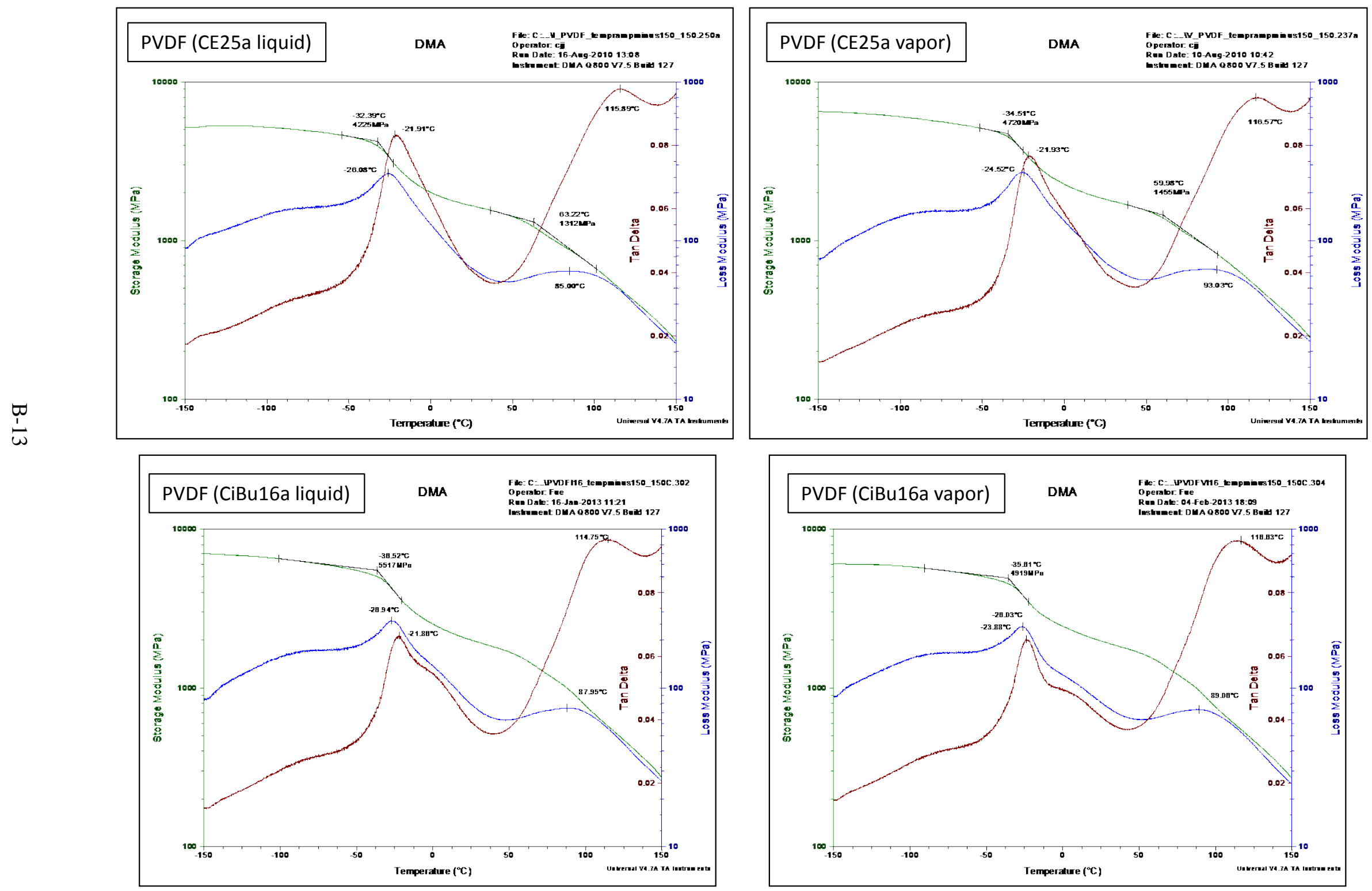
PVDF DMA Results for CiBu24a Exposures
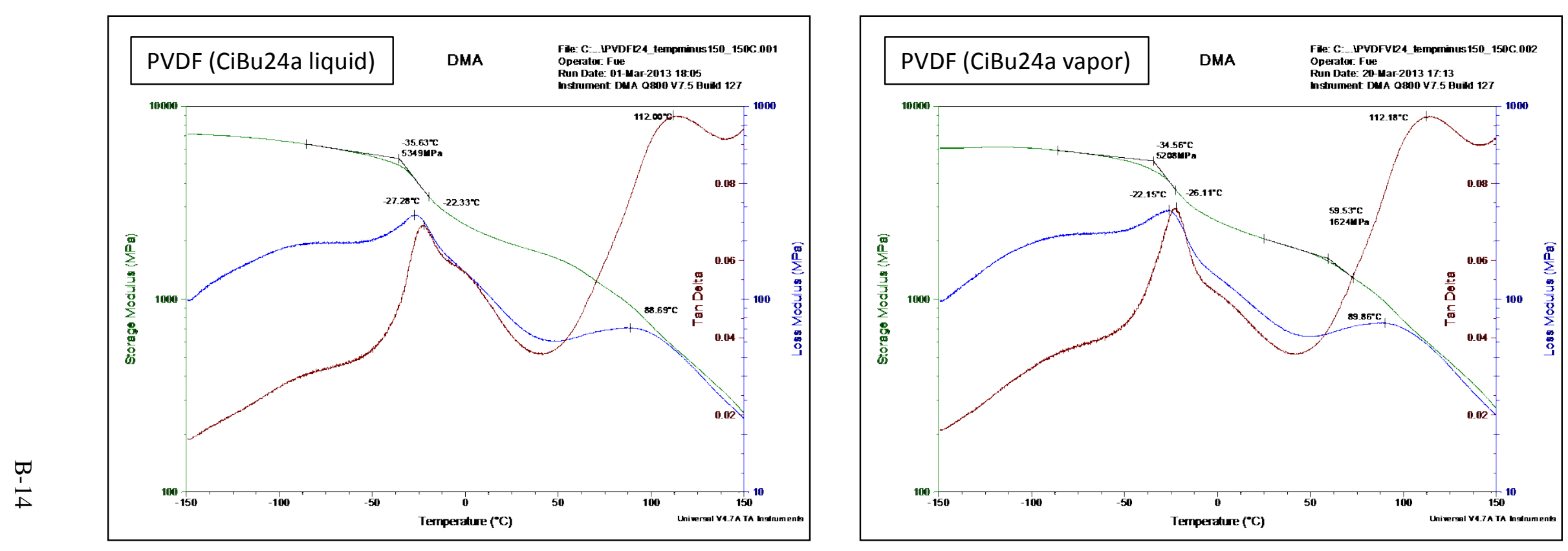
POM DMA Results for Control and Fuel C Exposures
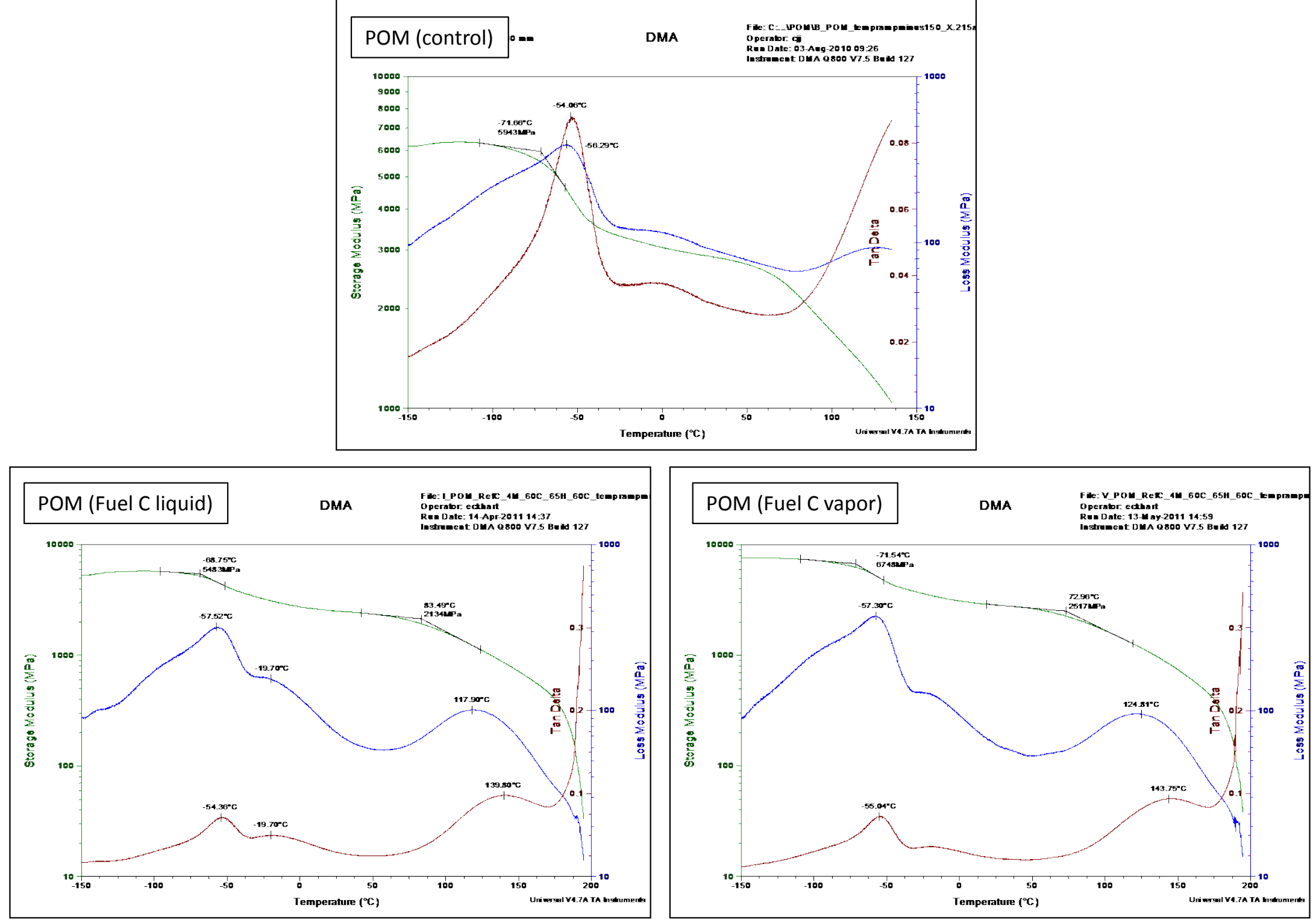


\section{POM DMA Results for CE25a and CiBu16a Exposures}
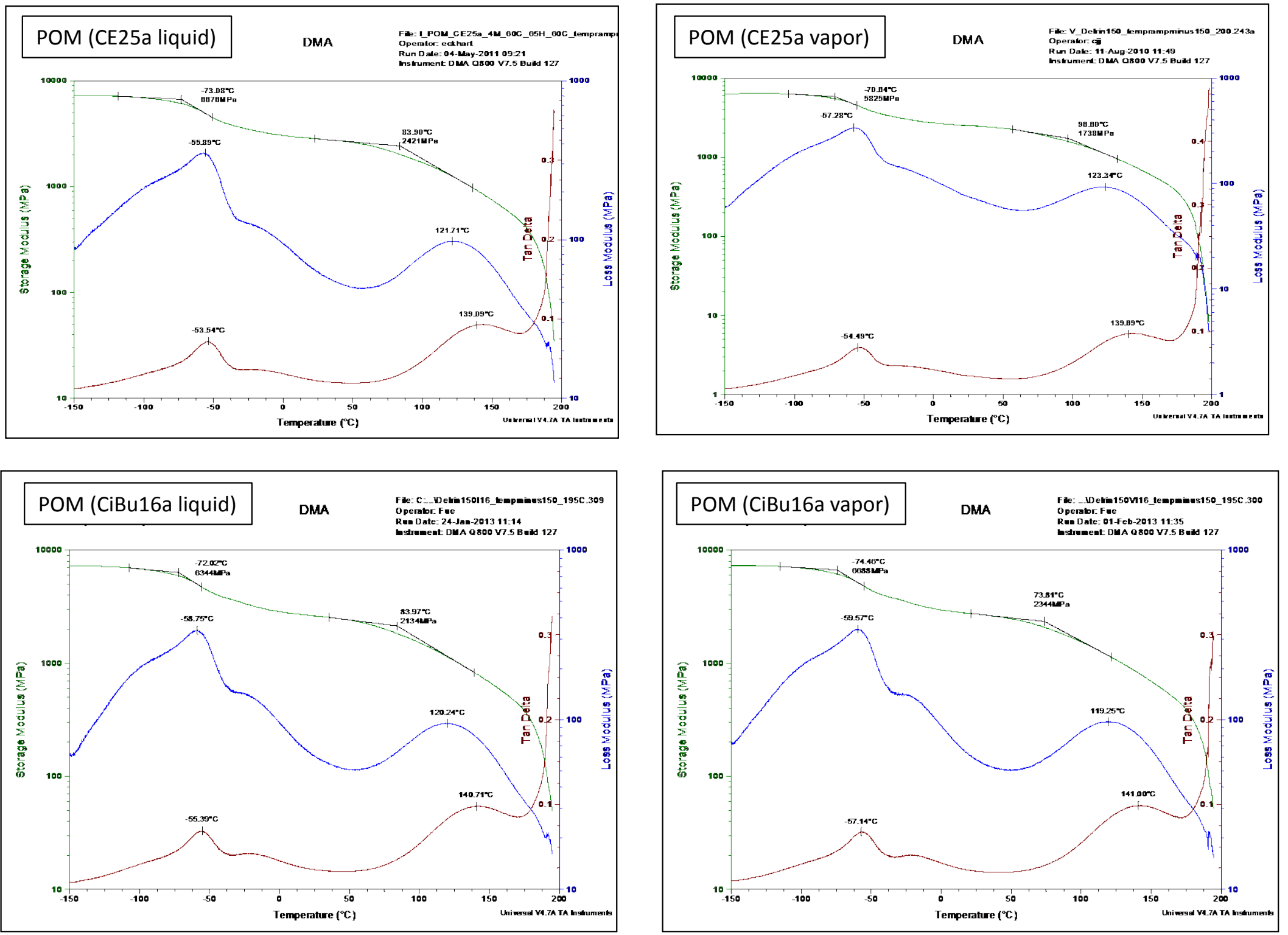
POM DMA Results for CE25a and CiBu16a Exposures
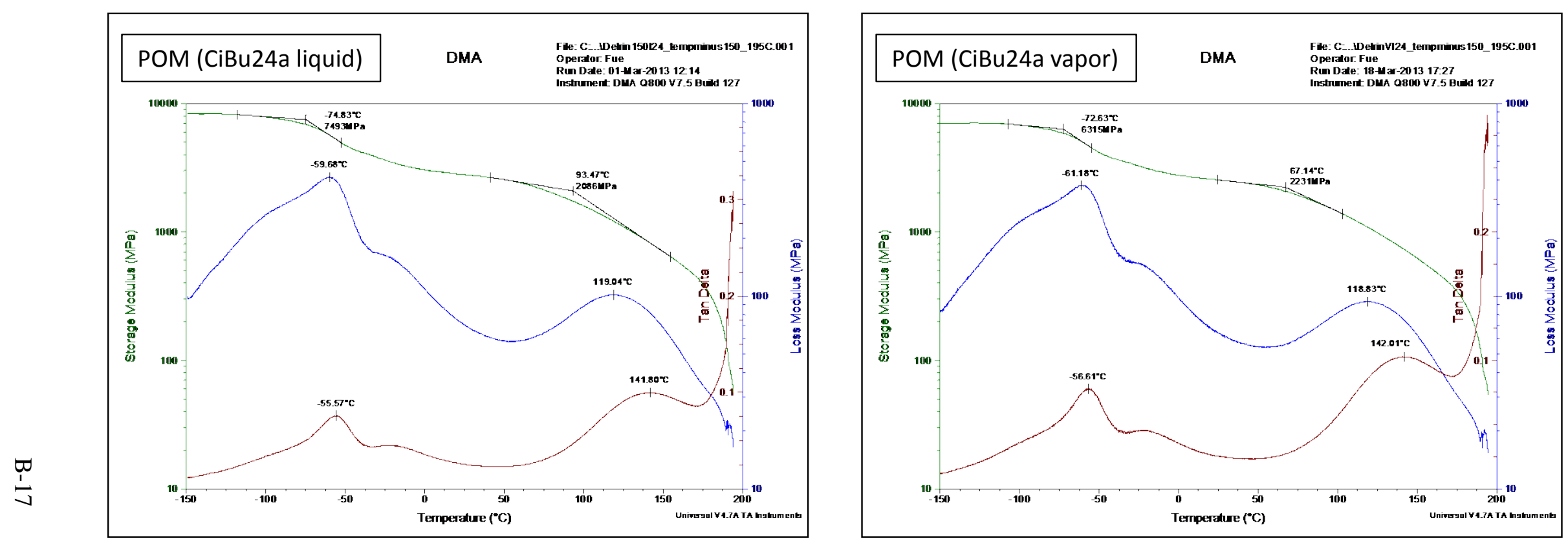
POM Copolymer DMA Results for Control and Fuel C Exposures
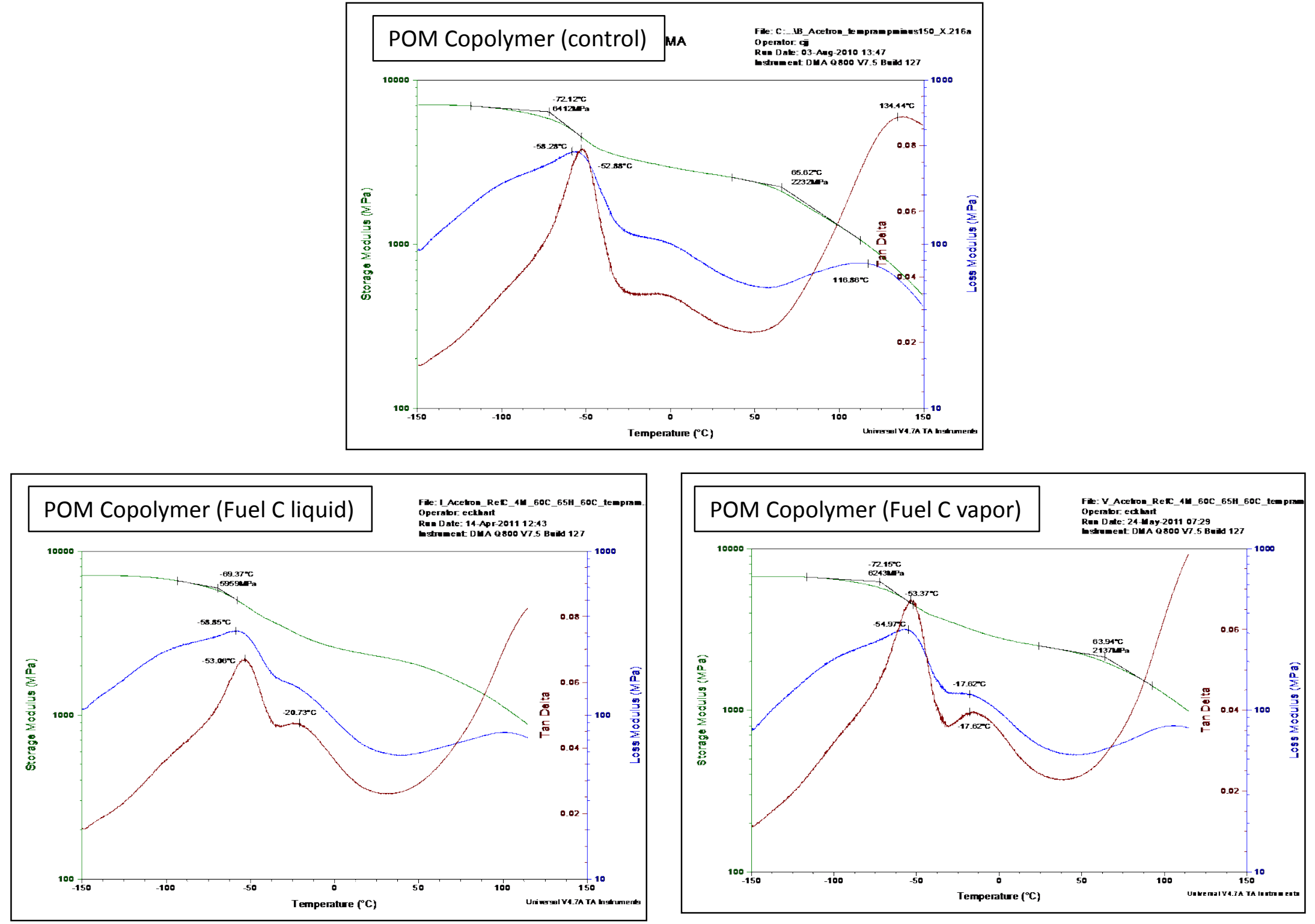


\section{POM Copolymer DMA Results for CE25a and CiBu16a Exposures}
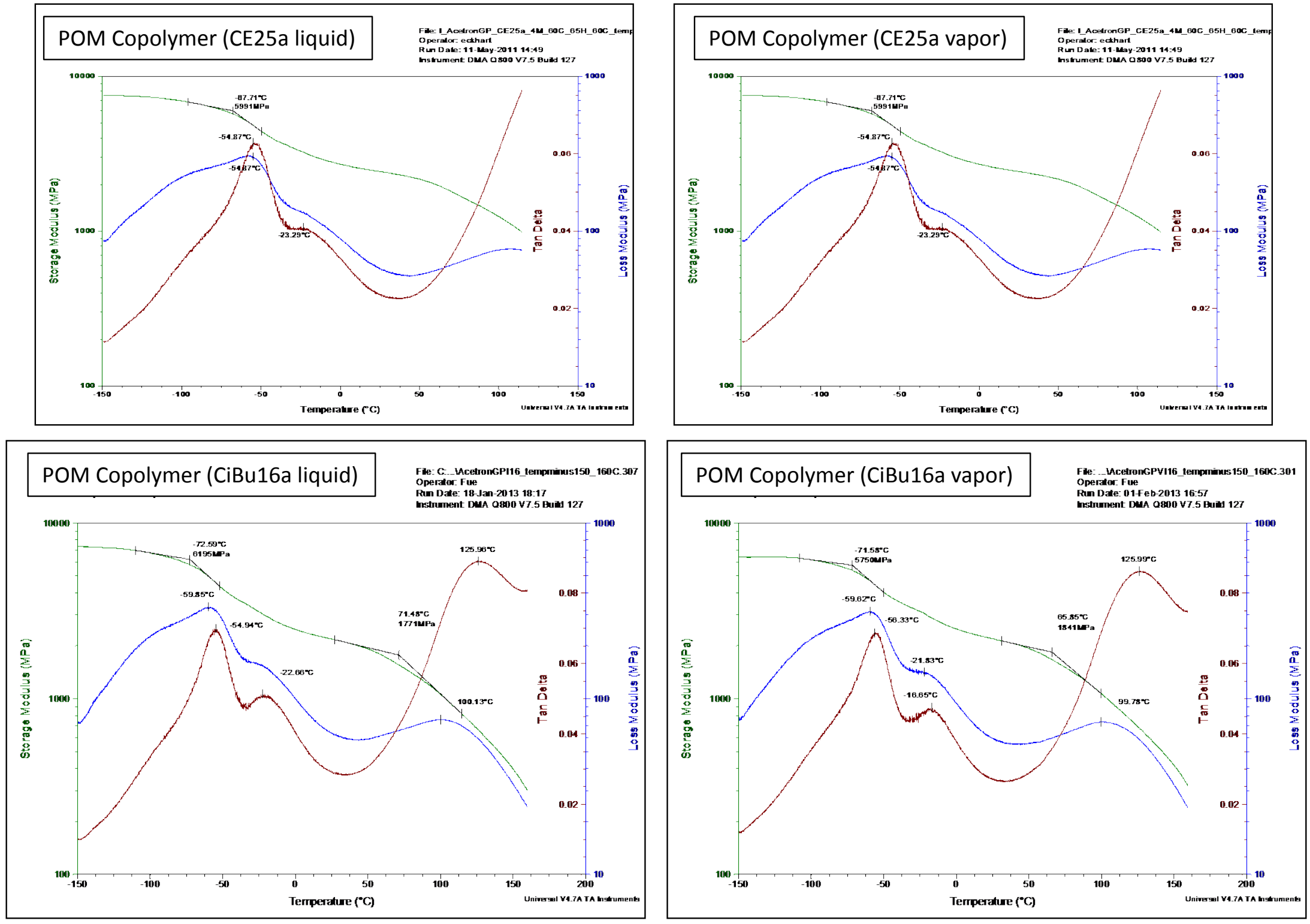
POM Copolymer DMA Results for CiBu24a Exposures
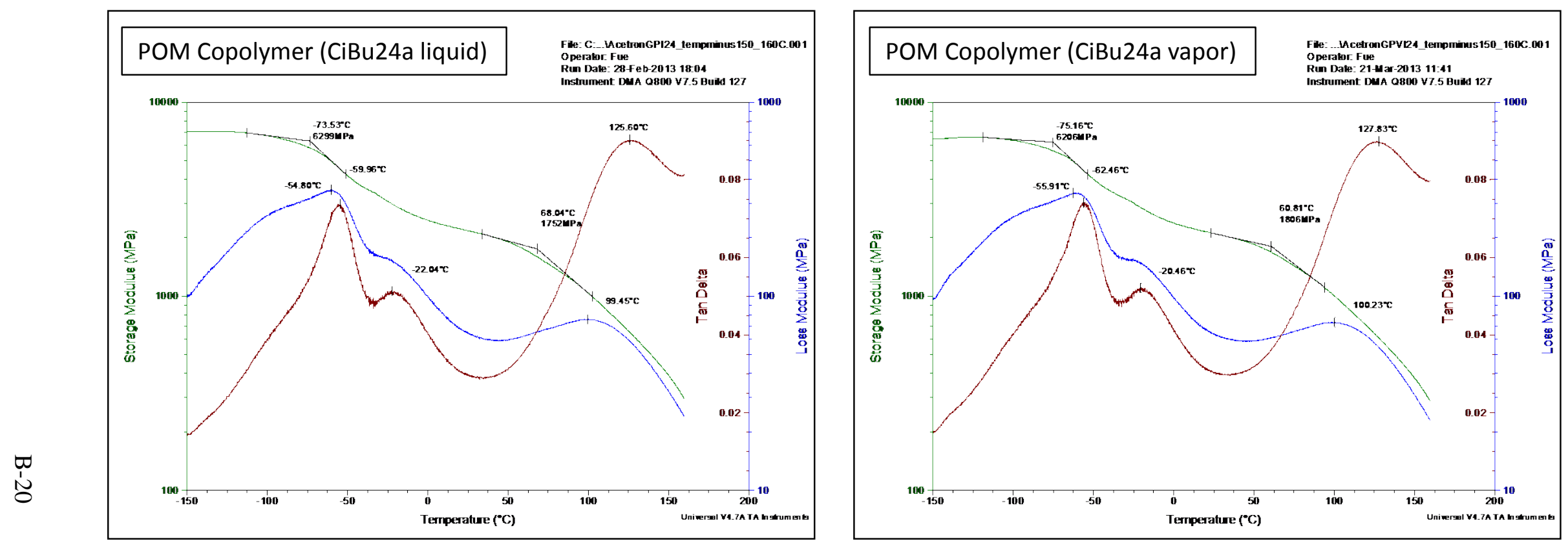


\section{PBT DMA Results for Control and Fuel C Exposures}
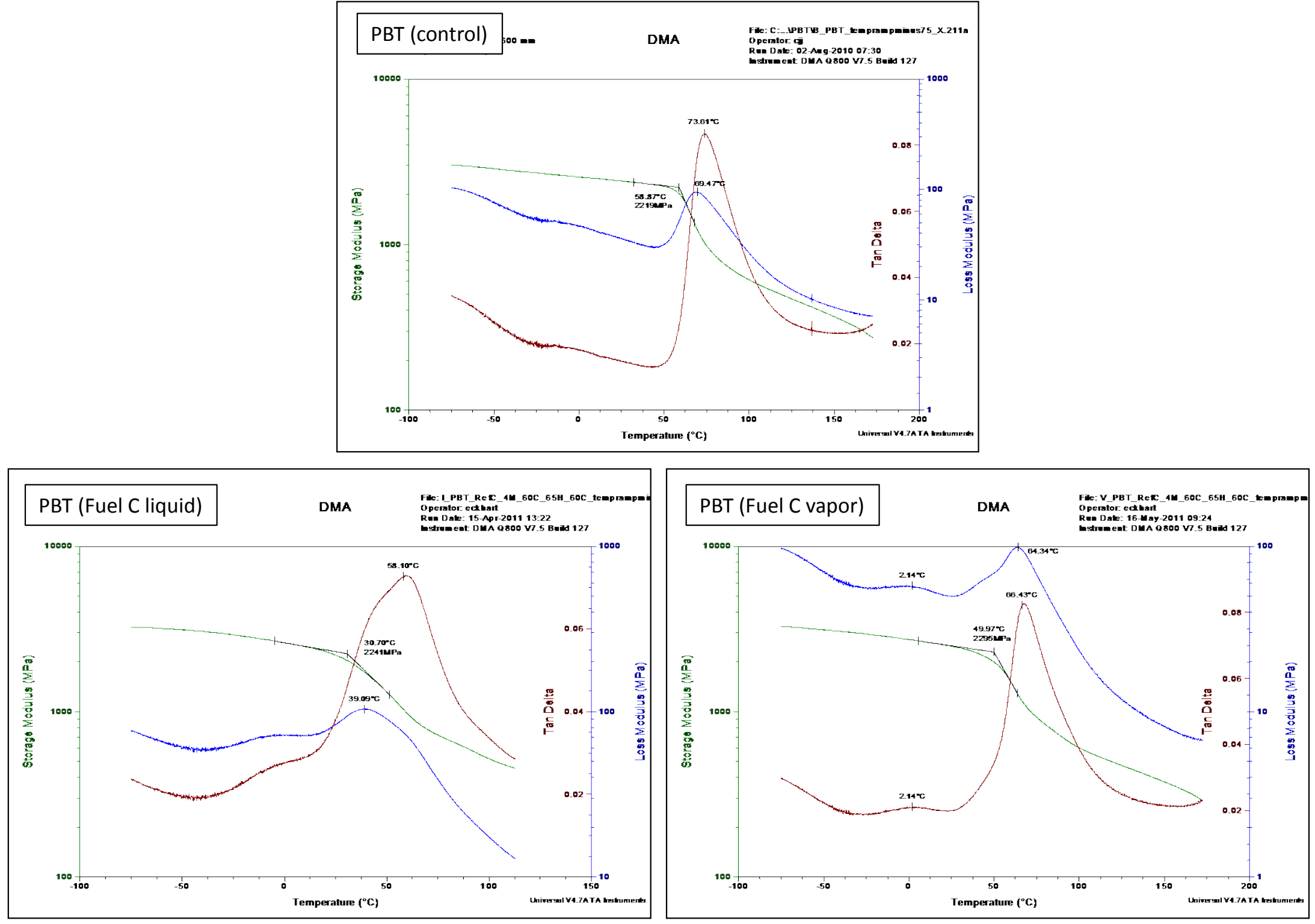
PBT DMA Results for CE25a and CiBu16a Exposures
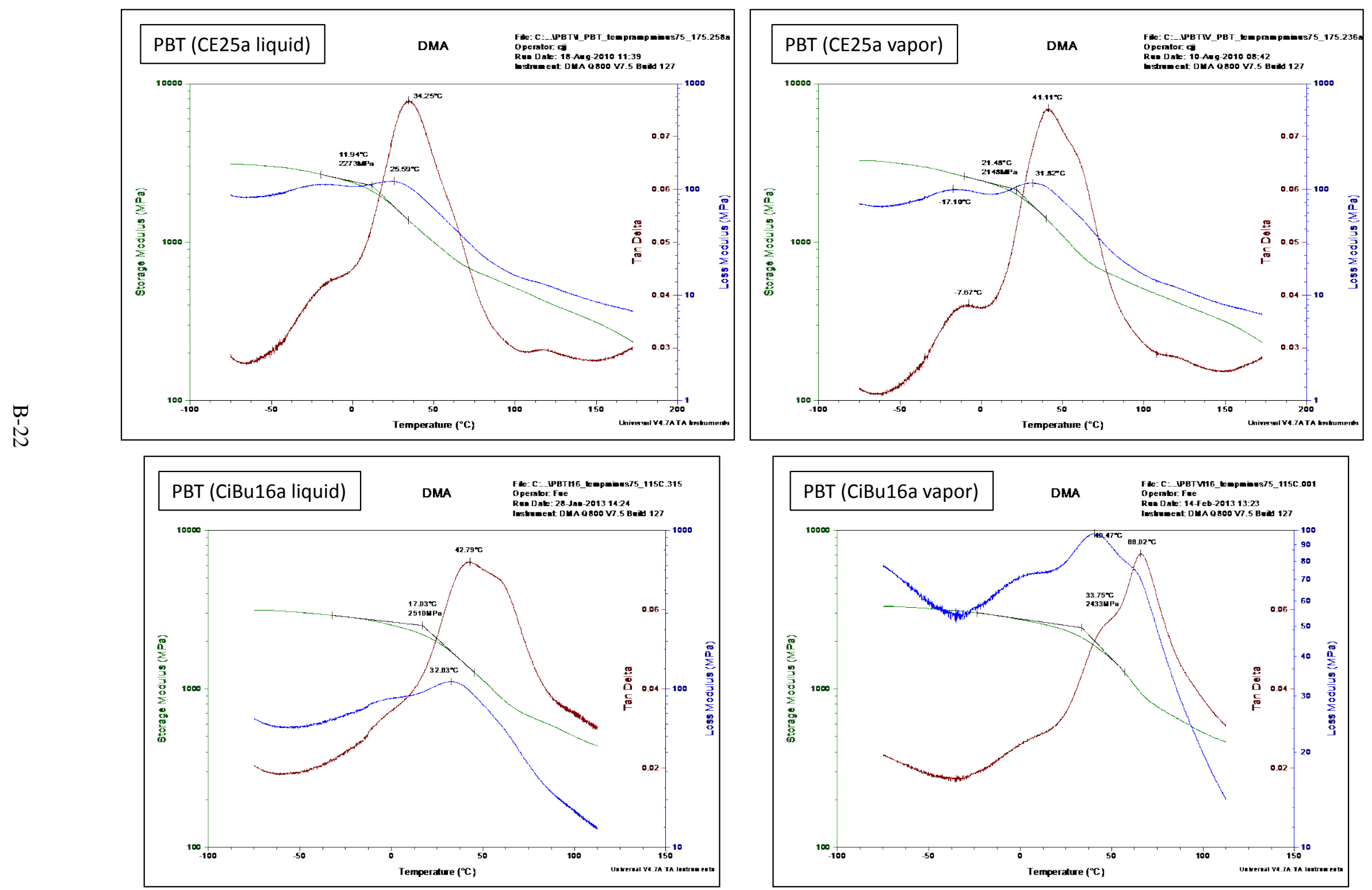
PBT DMA Results for CE25a and CiBu16a Exposures
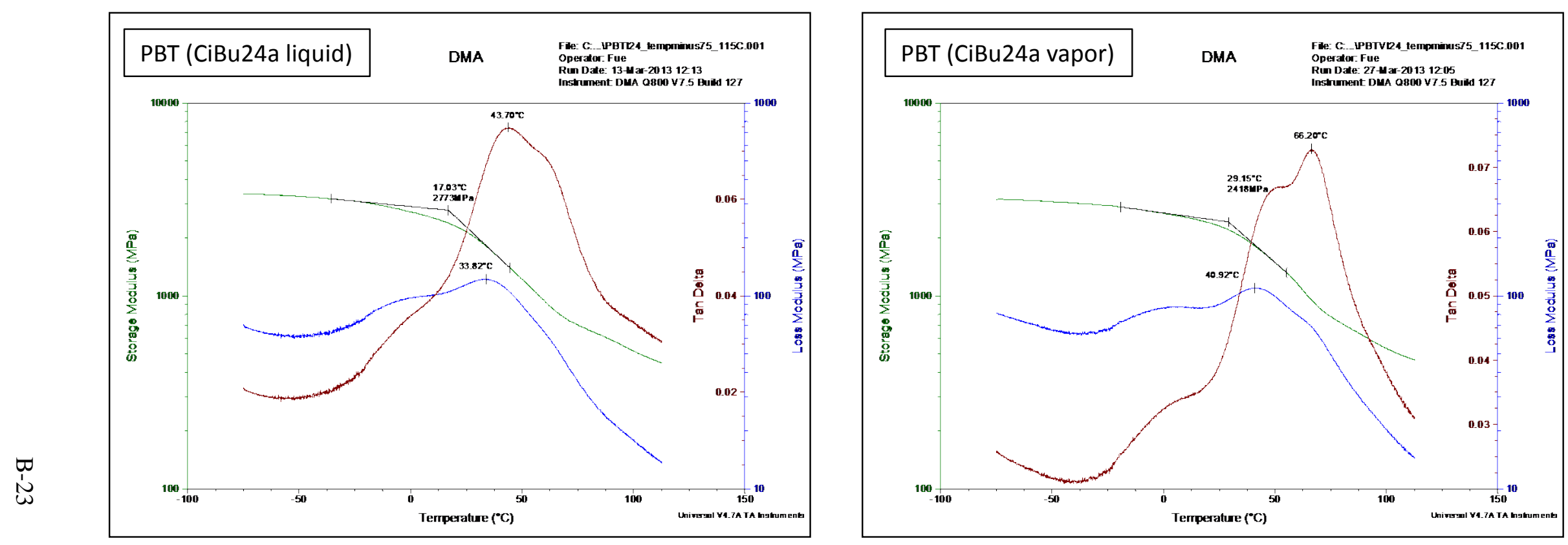


\section{Nylon 12 DMA Results for Control and Fuel C Exposures}

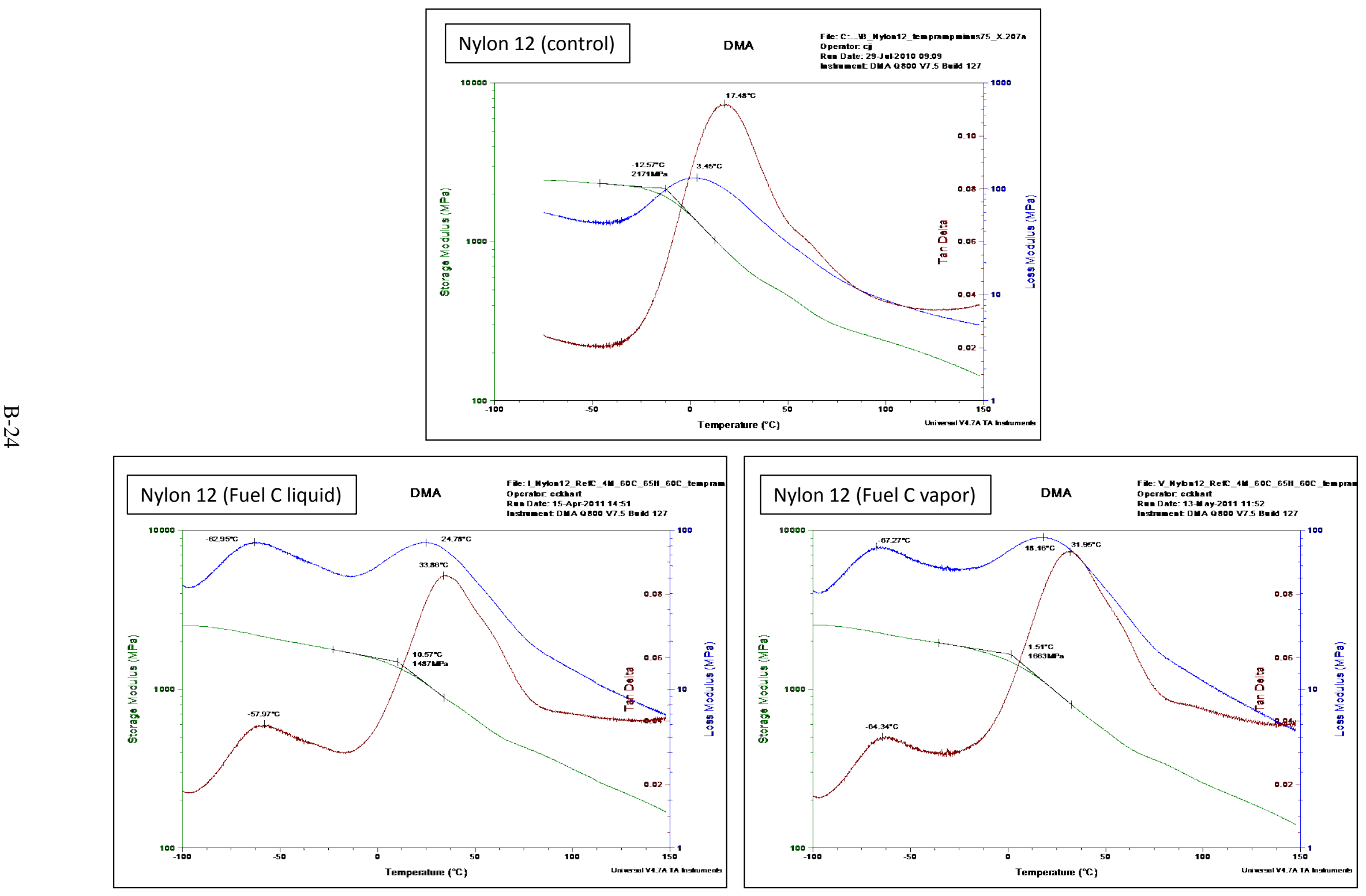




\section{Nylon 12 DMA Results for CE25a and CiBu16a Exposures}
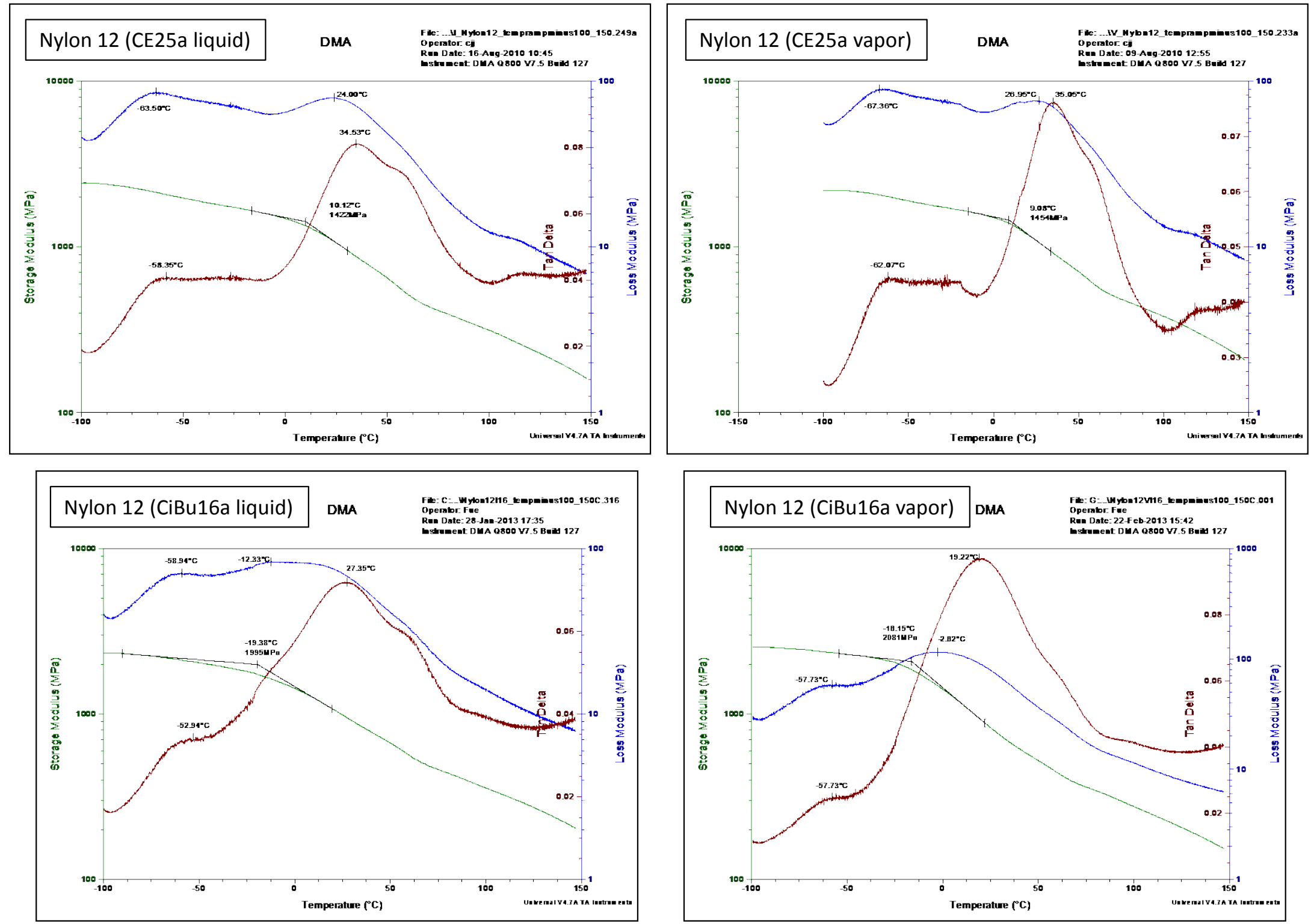
Nylon 12 DMA Results for CiBu24a Exposures
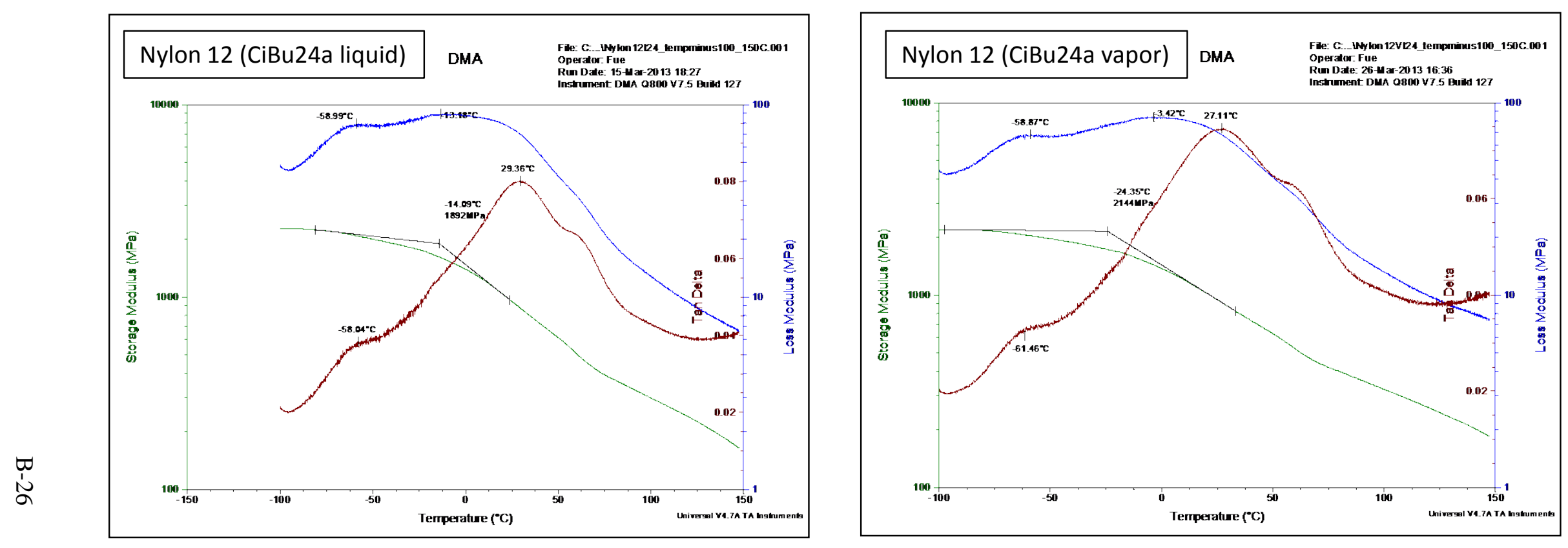


\section{Nylon 6 DMA Results for Control and Fuel C Exposures}
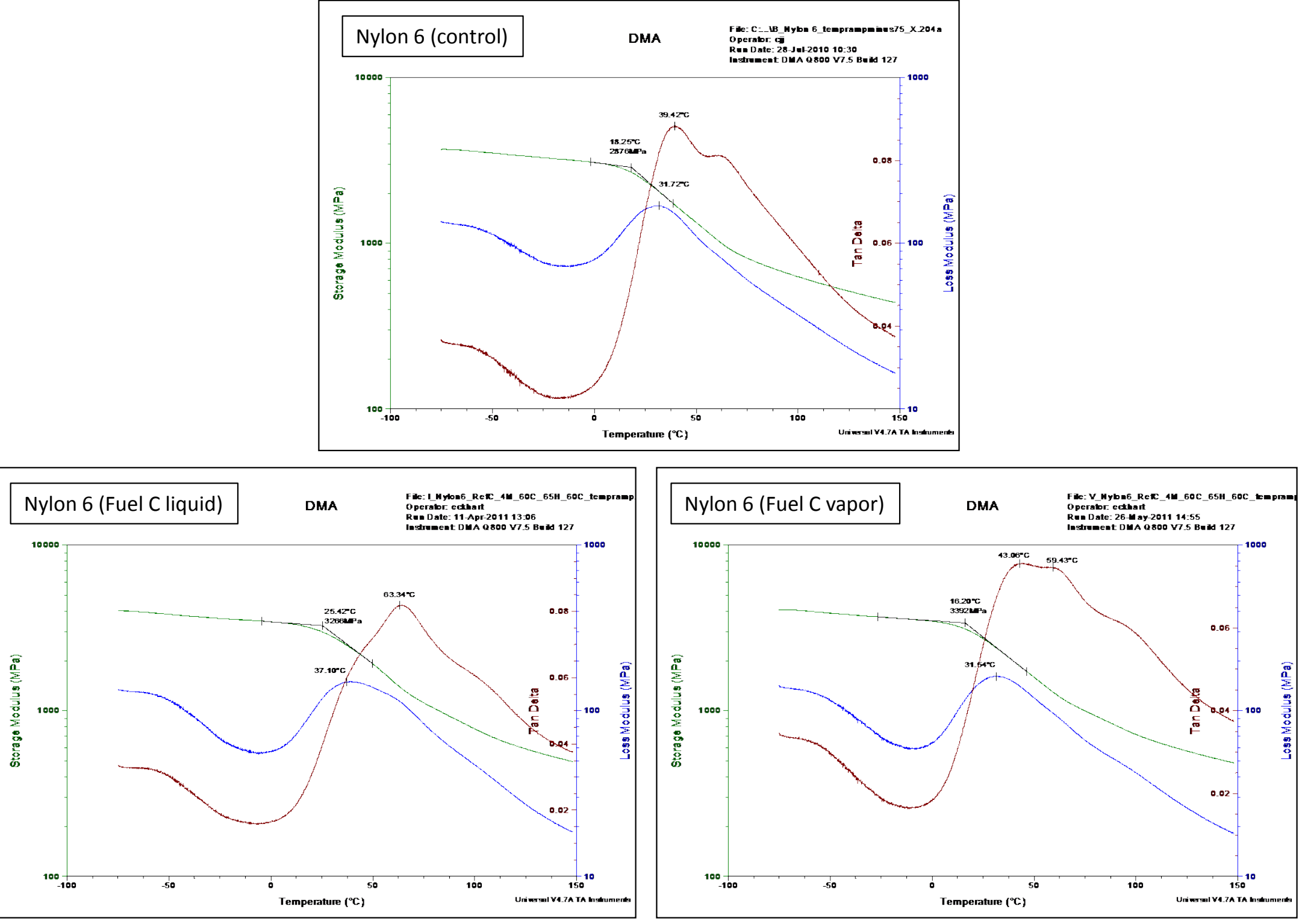
Nylon 6 DMA Results for CE25a and CiBu16a Exposures
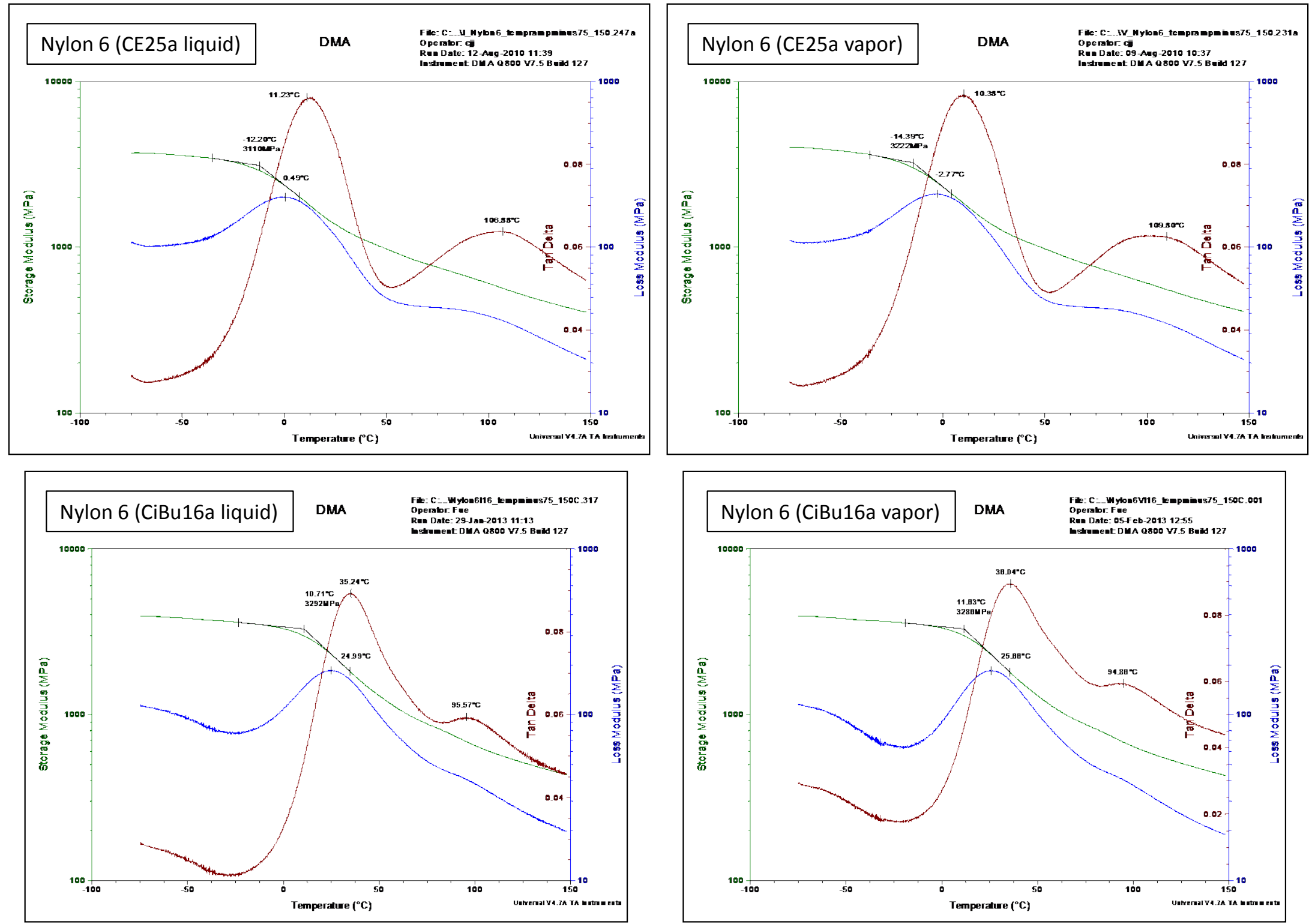
Nylon 6 DMA Results for CiBu24a Exposures
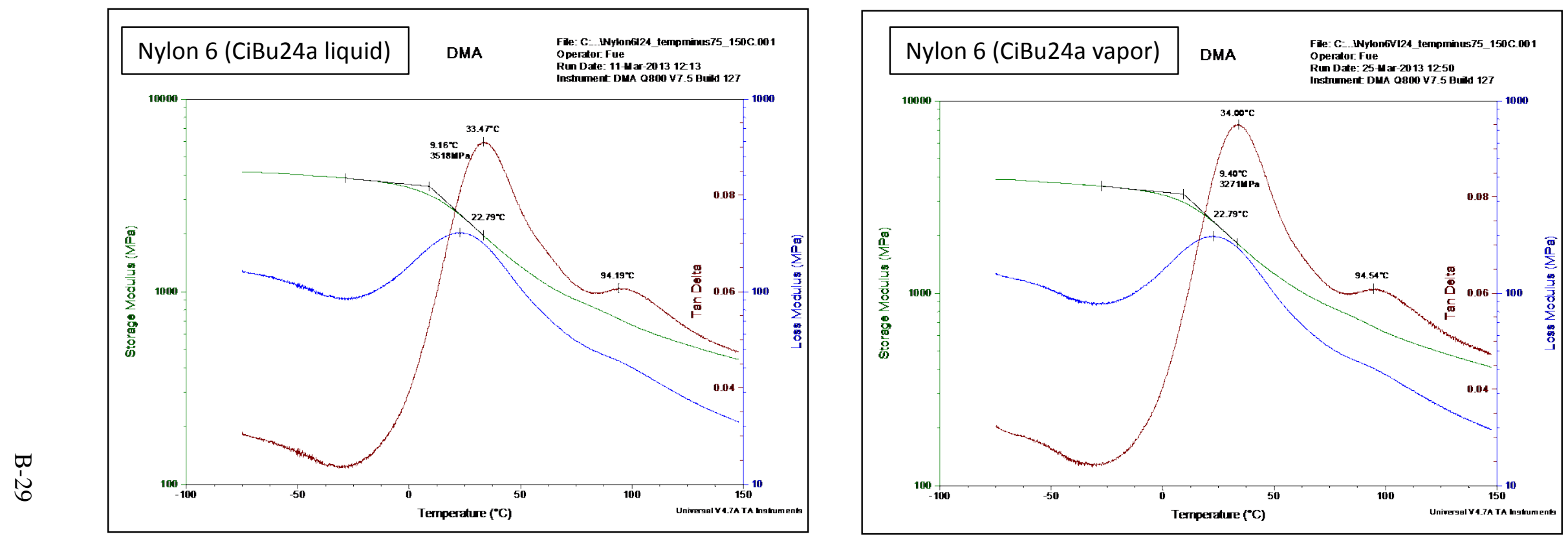


\section{Nylon 6,6 DMA Results for Control and Fuel C Exposures}
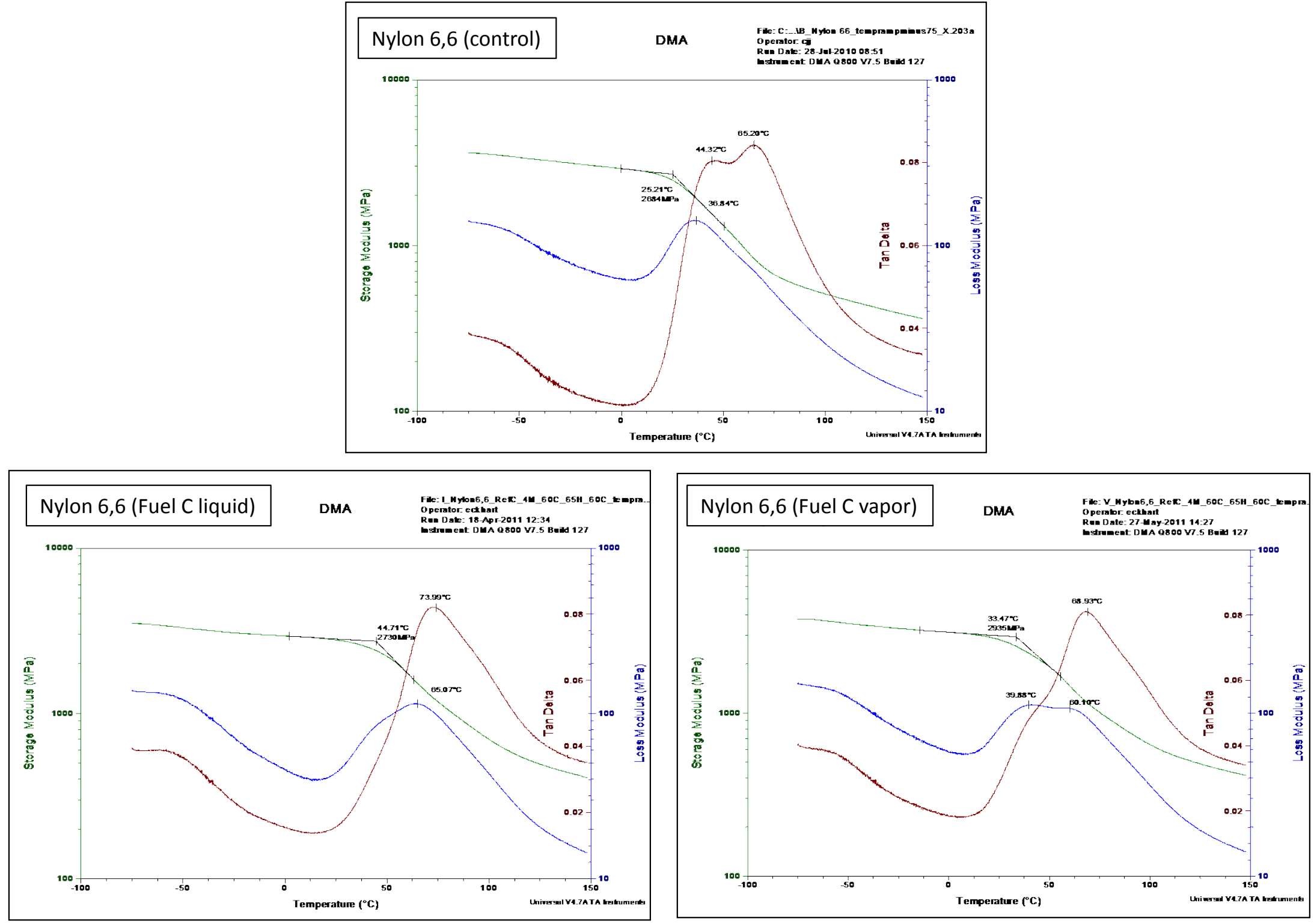
Nylon 6,6 DMA Results for CE25a and CiBu16a Exposures
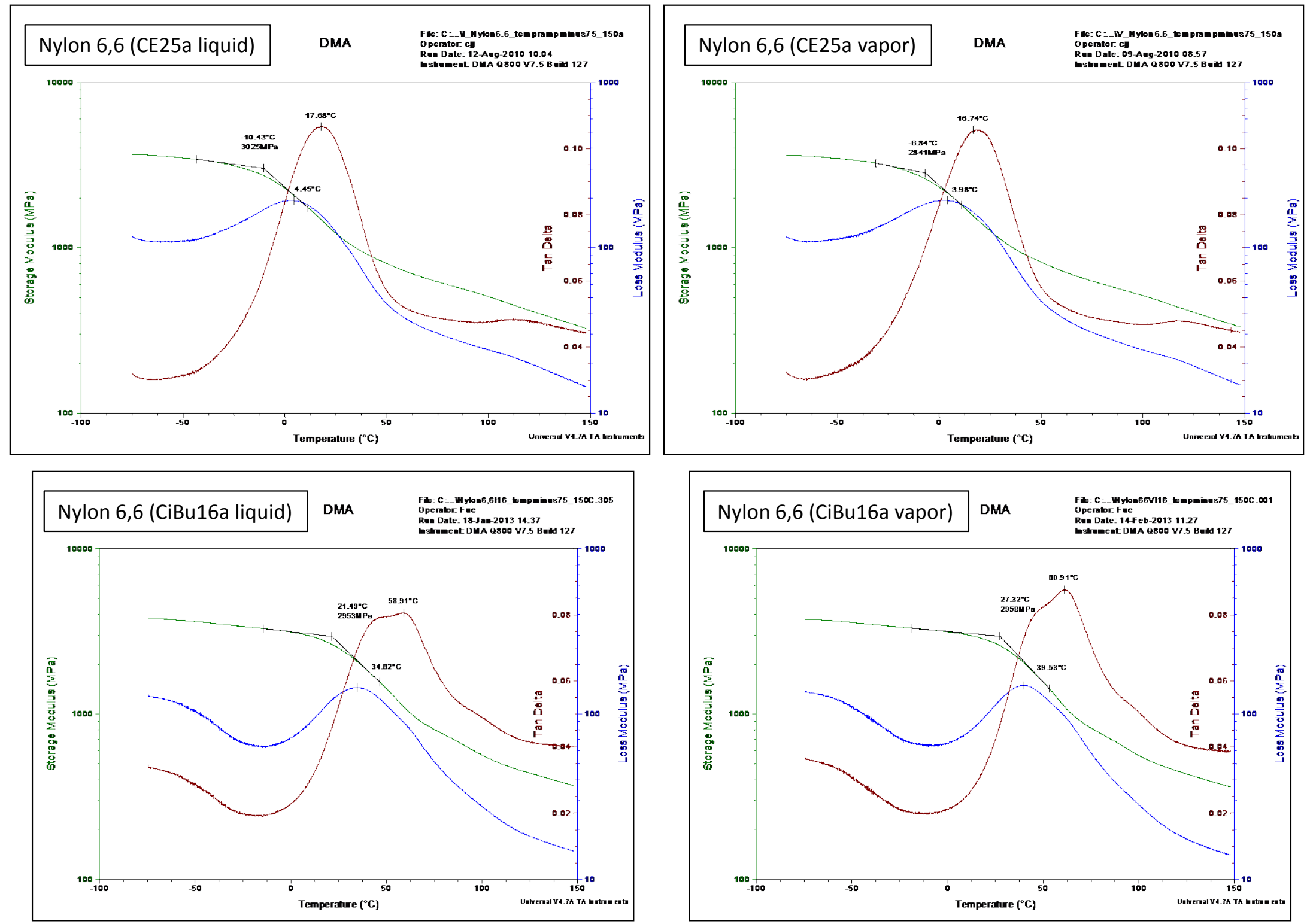
Nylon 6,6 DMA Results for CiBu24a Exposures
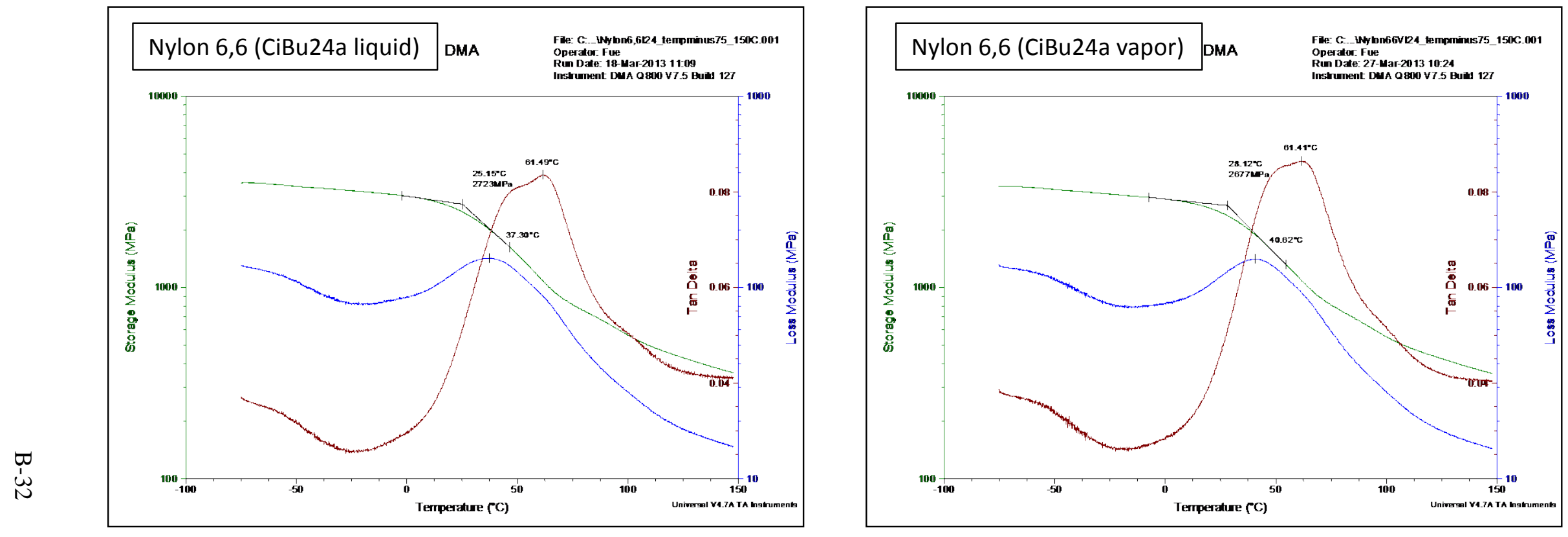
Nylon 11 DMA Results for Control and Fuel C Exposures
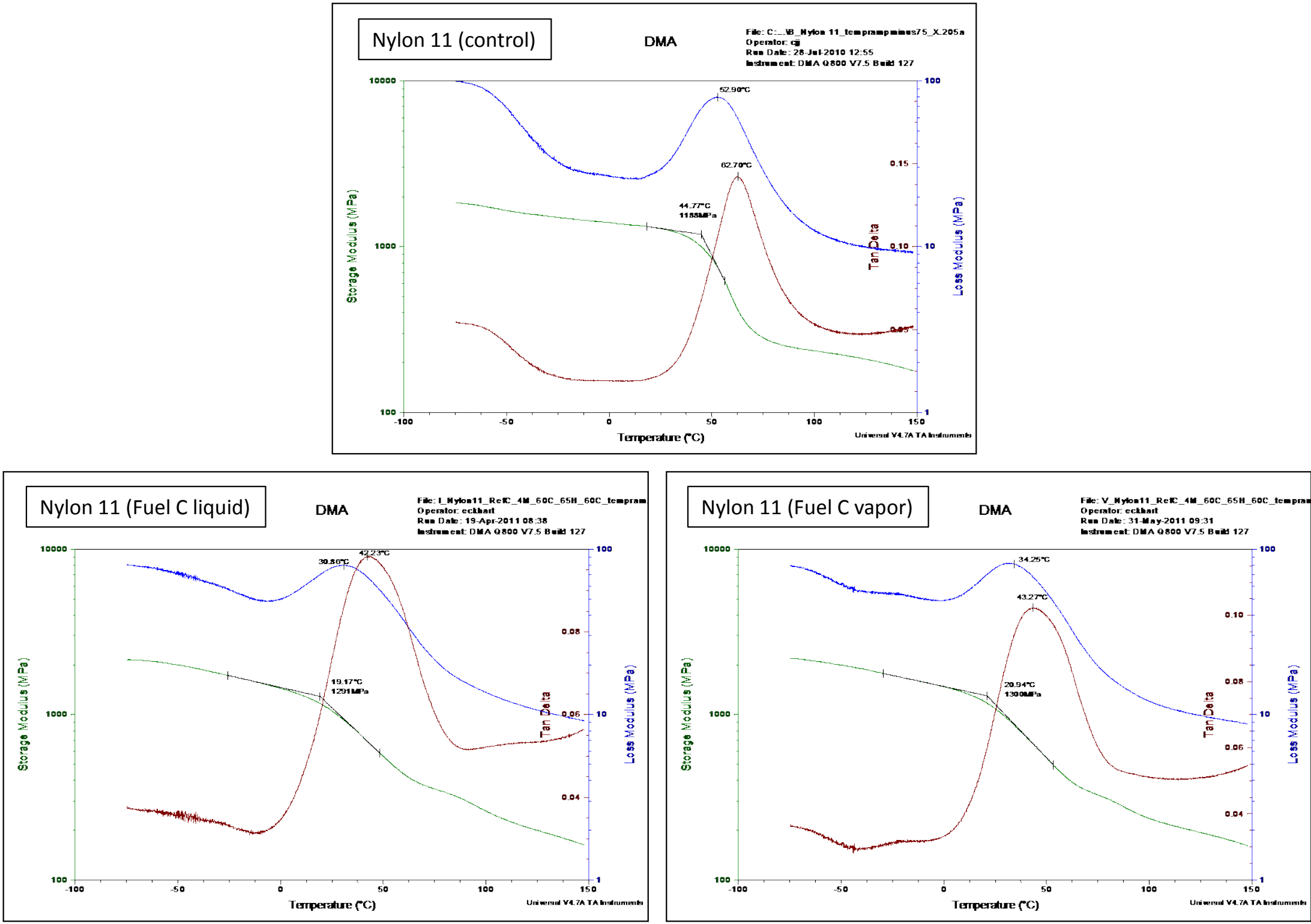
Nylon 11 DMA Results for CE25a and CiBu16a Exposures
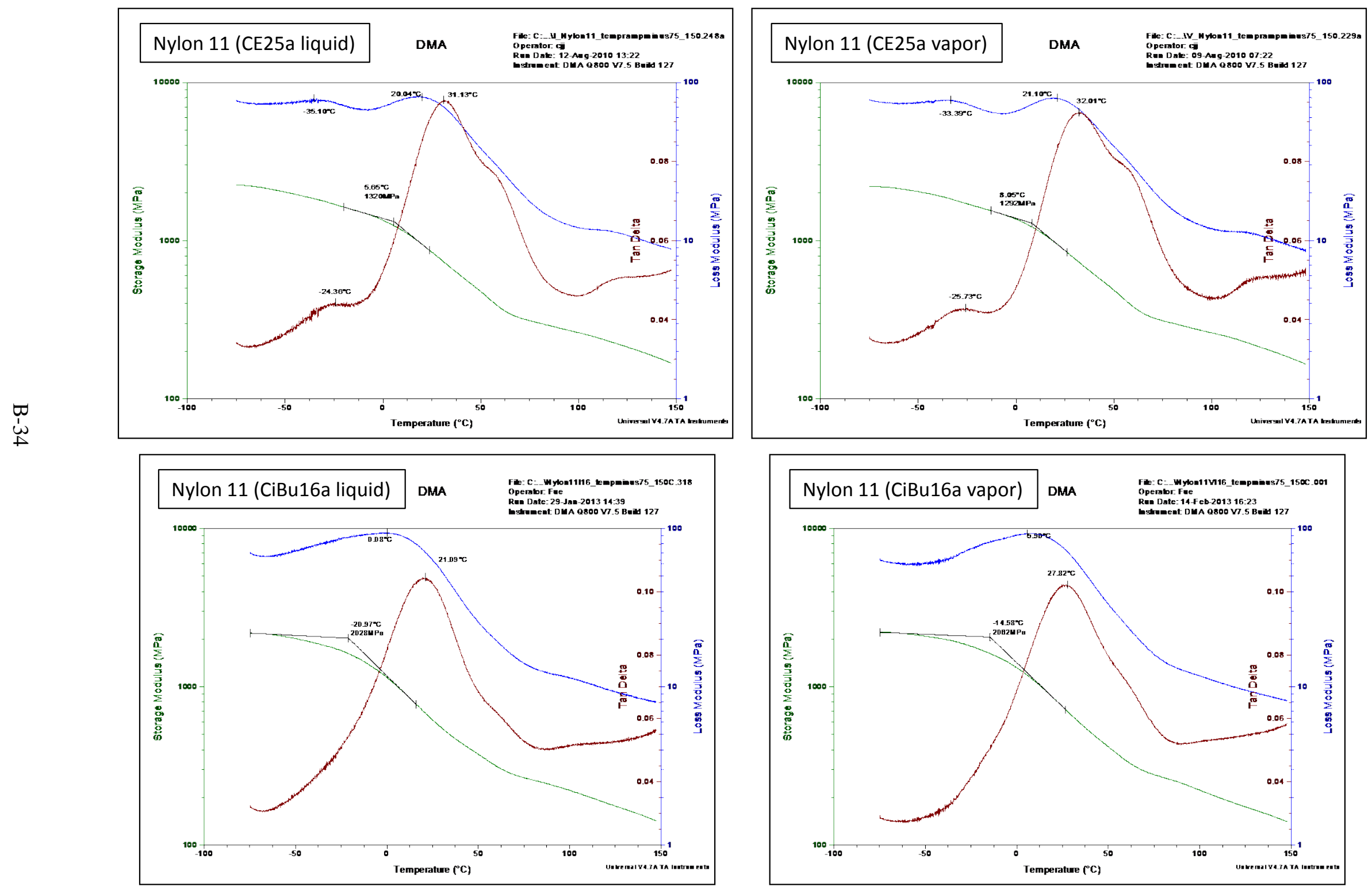
Nylon 11 DMA Results for CiBu24a Exposures
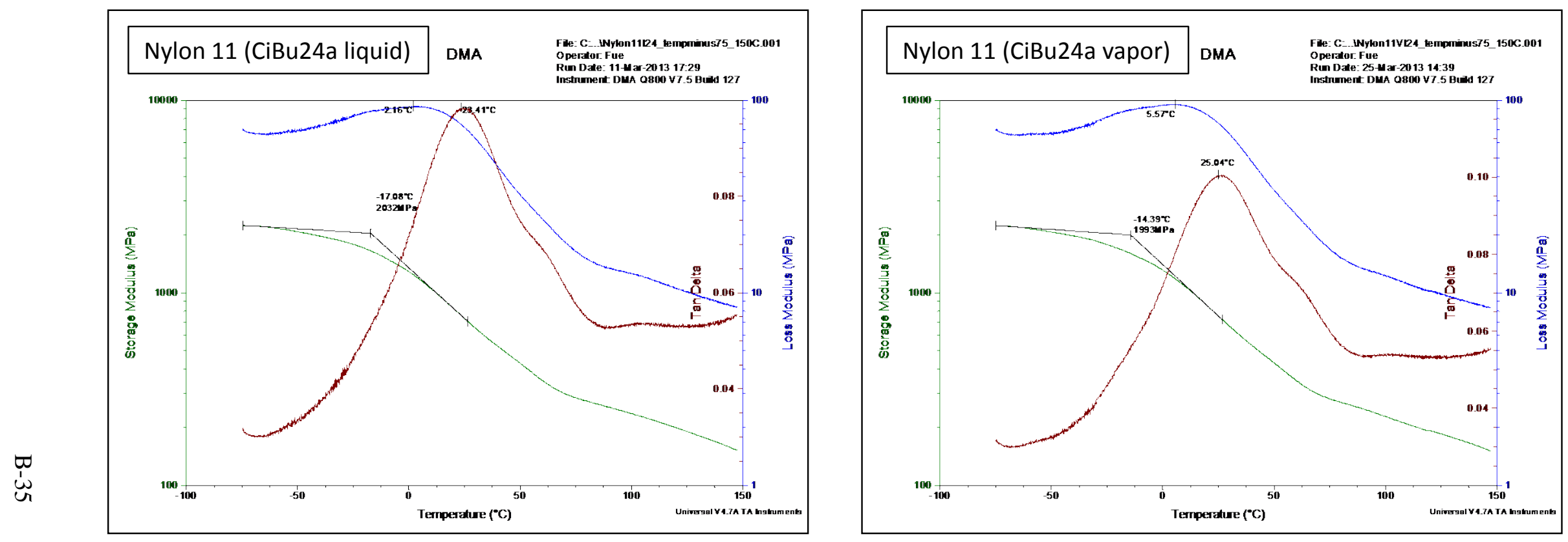


\section{HDPE DMA Results for Control and Fuel C Exposures}
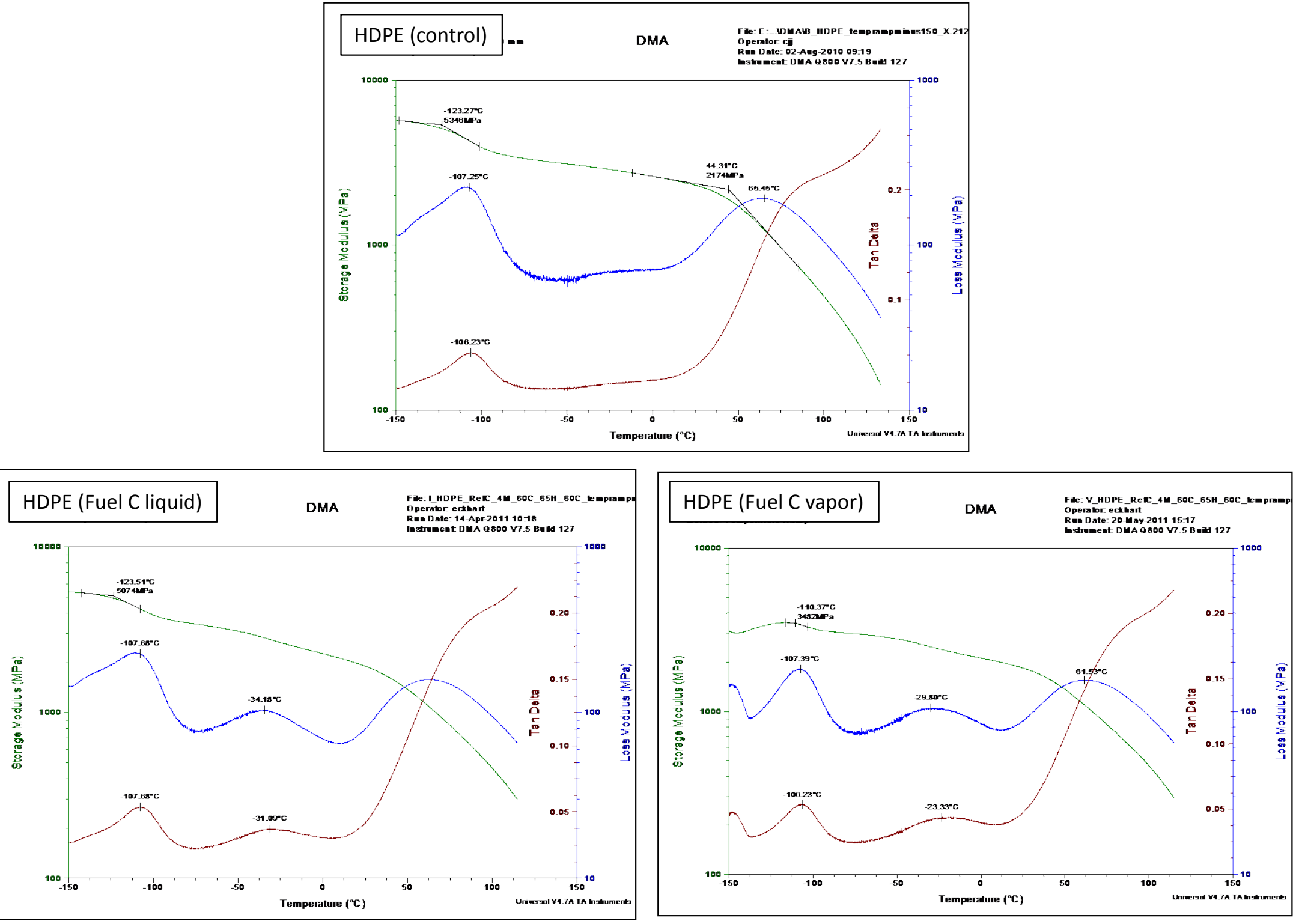


\section{HDPE DMA Results for CE25a and CiBu16a Exposures}
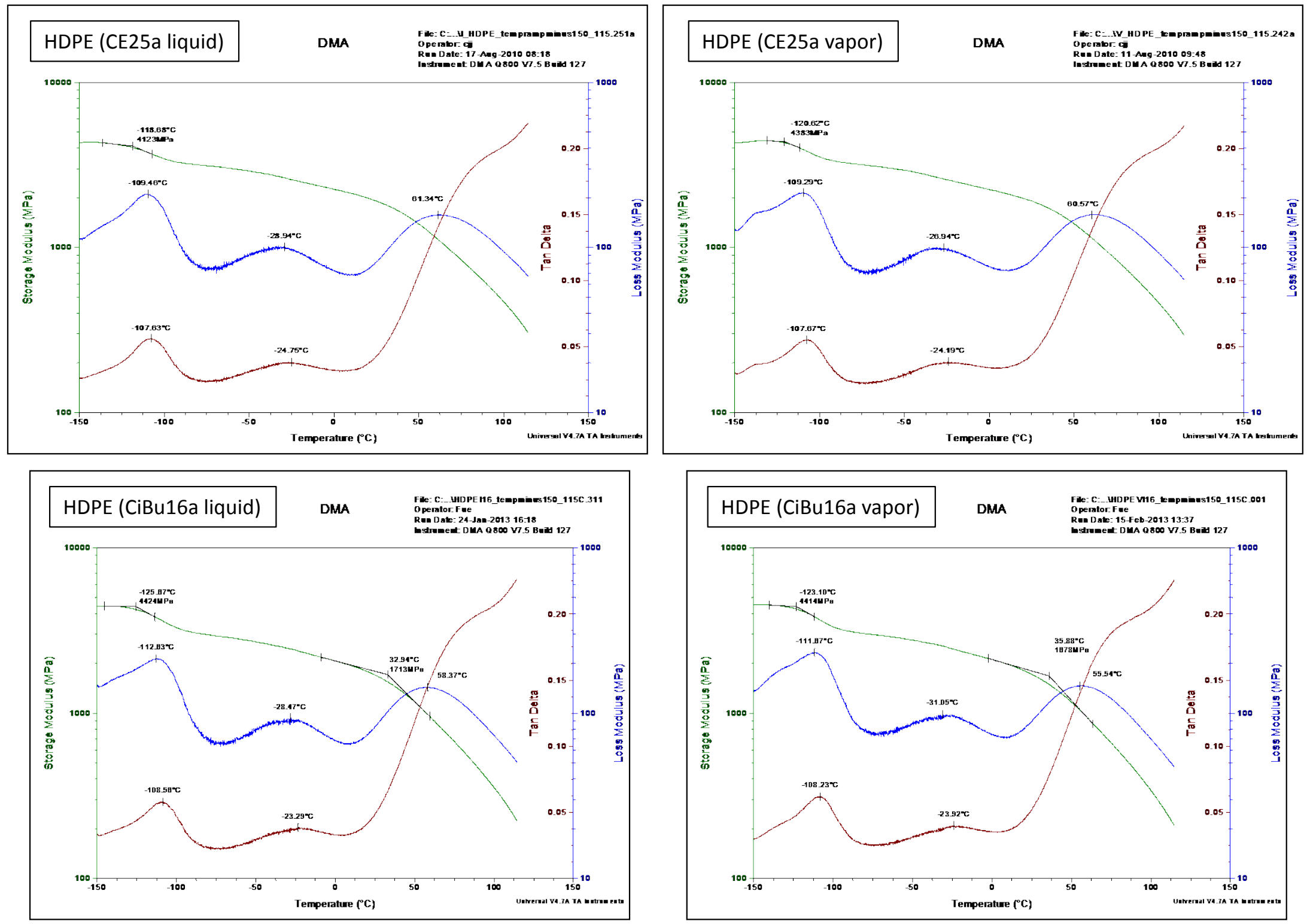


\section{HDPE DMA Results for CiBu24a Exposures}
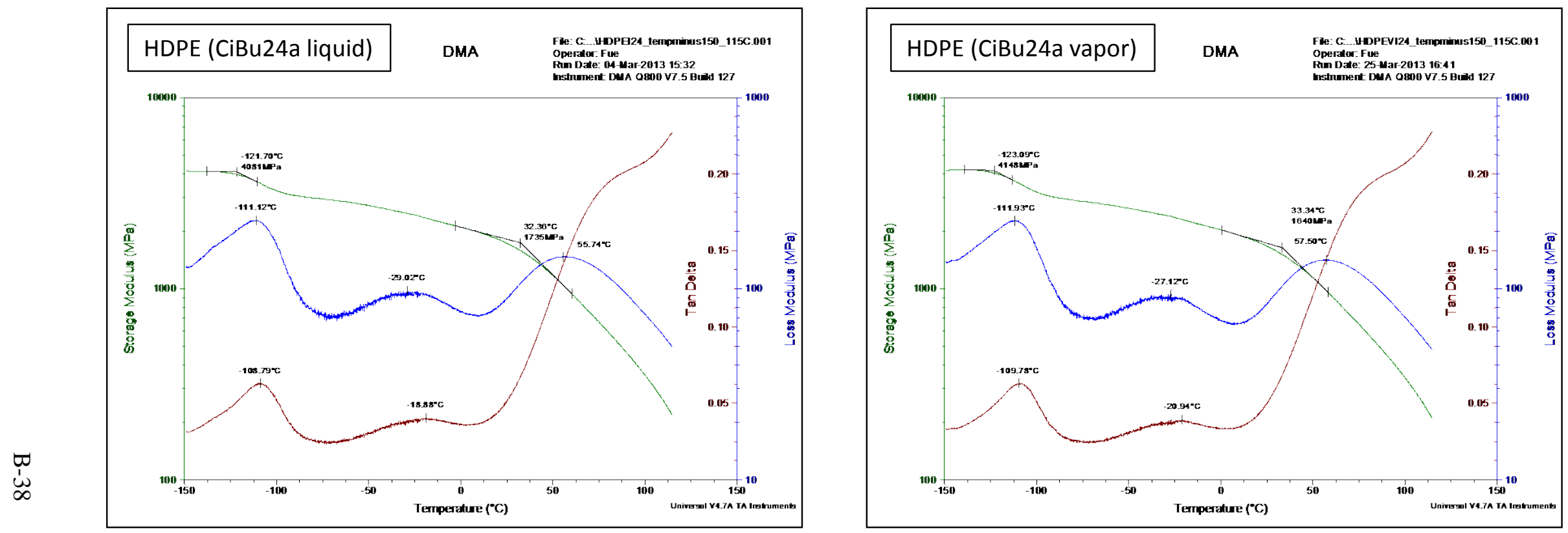


\section{PP DMA Results for Control and Fuel C Exposures}
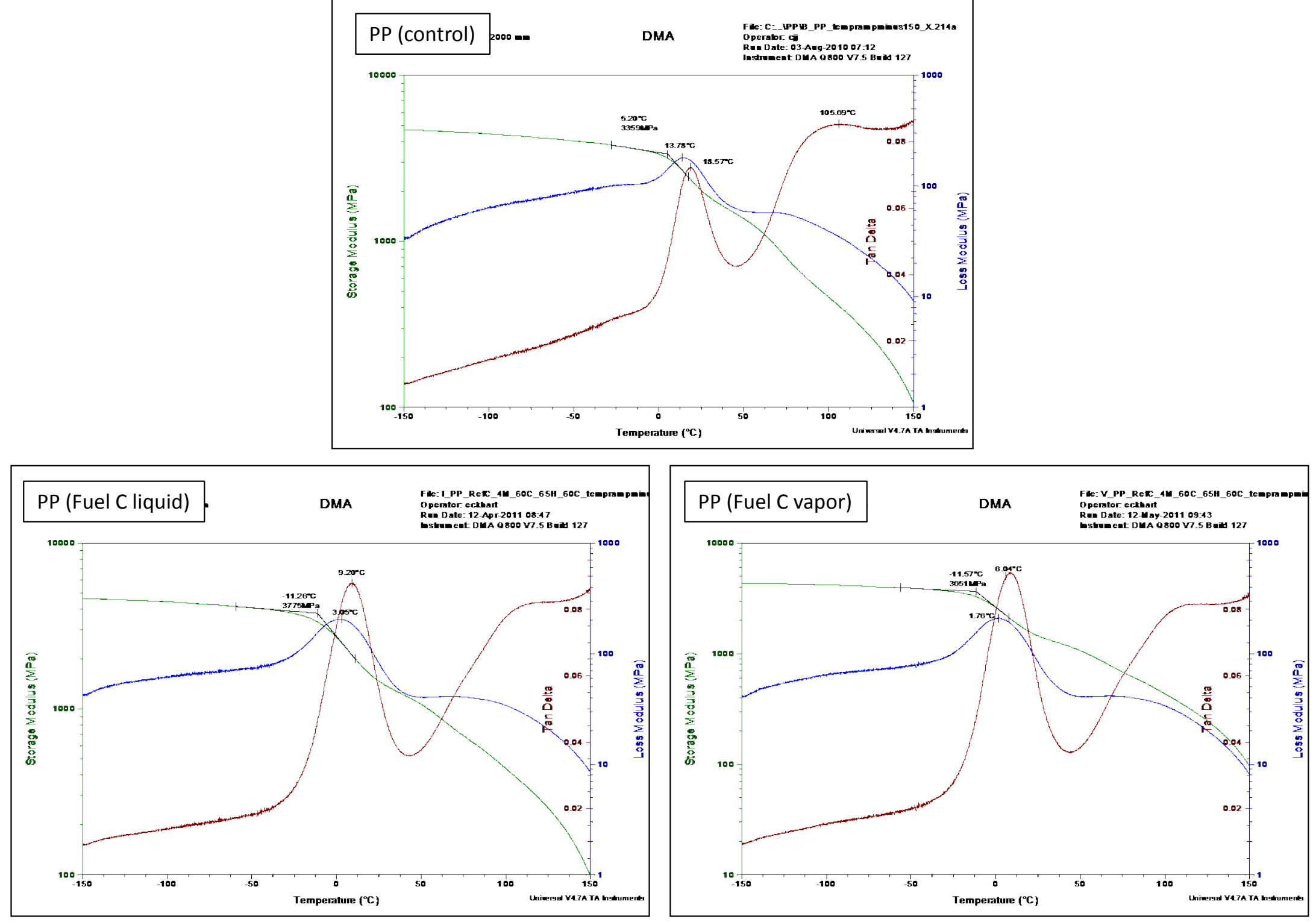
PP DMA Results for CE25a and CiBu16a Exposures
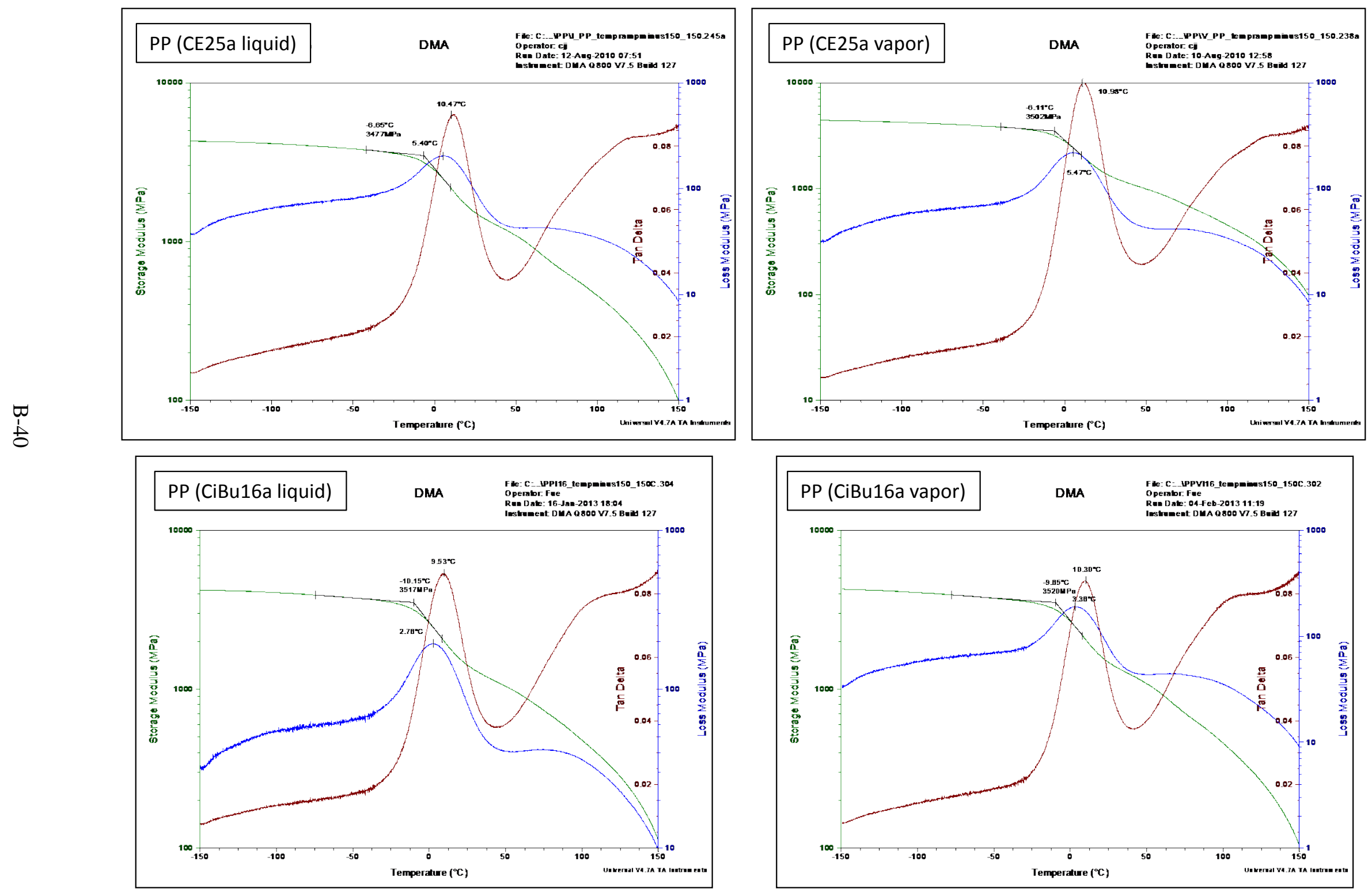
PP DMA Results for CiBu24a Exposures
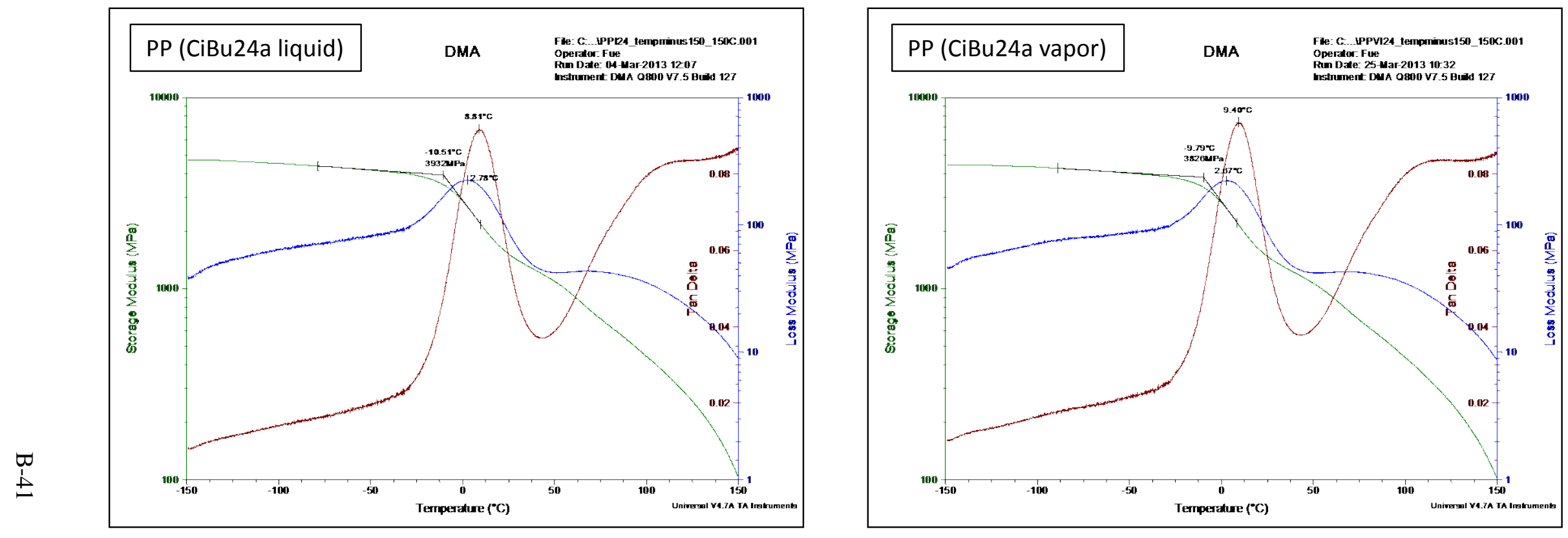


\section{PETG DMA Results for Control and Fuel C Exposures}
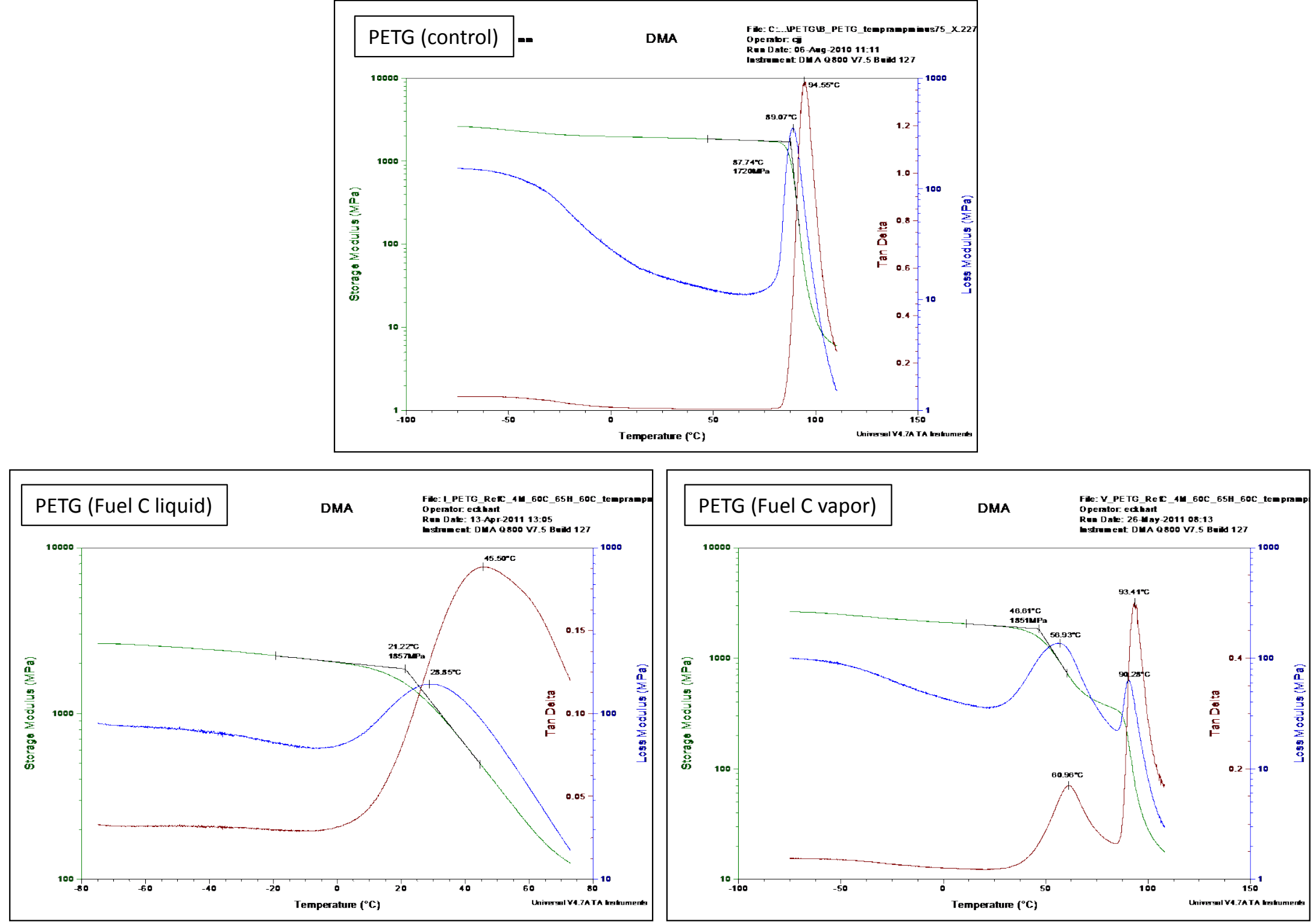


\section{PETG DMA Results for CE25a and CiBu16a Exposures}
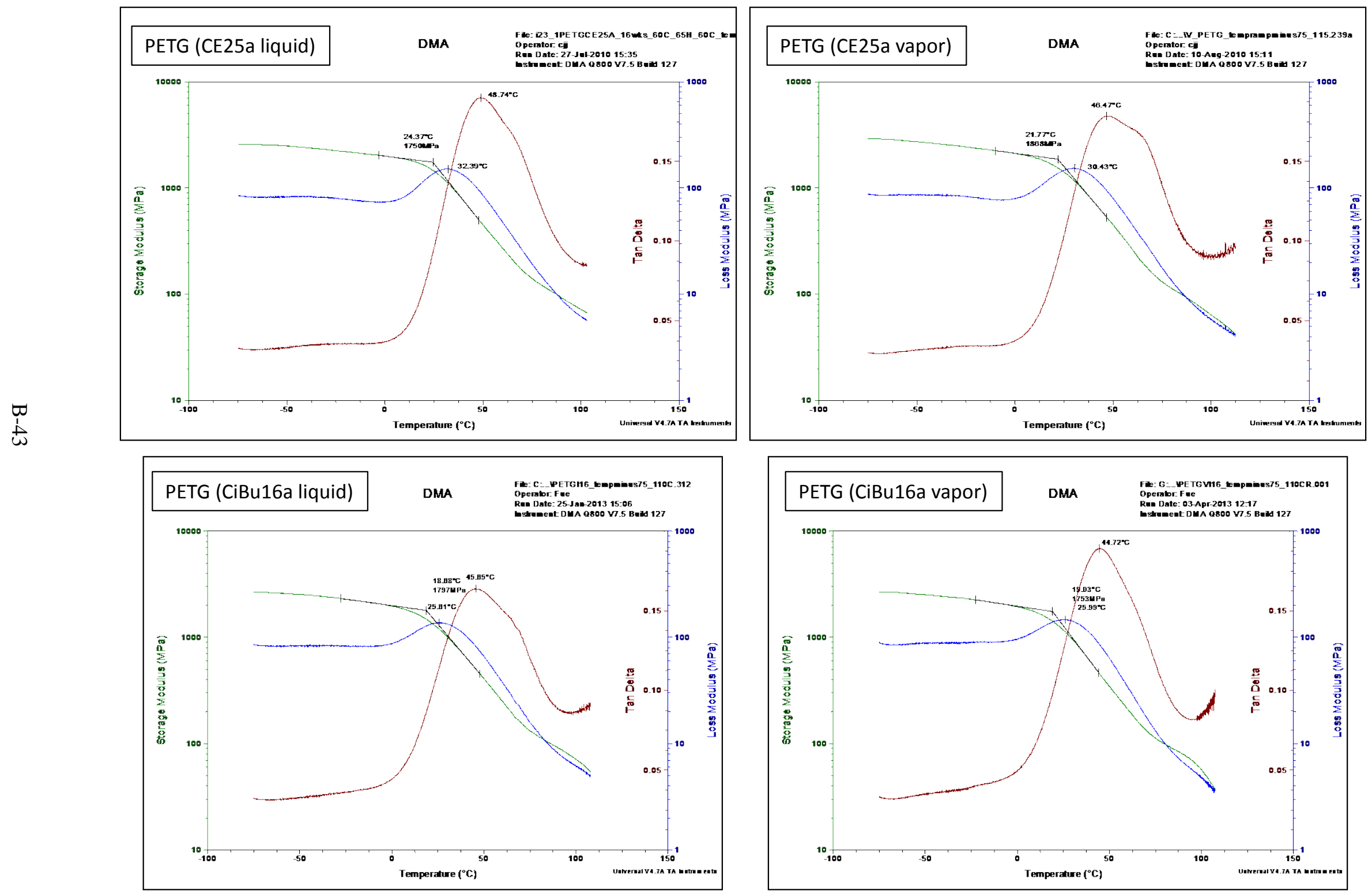
PETG DMA Results for CiBu24a Exposures
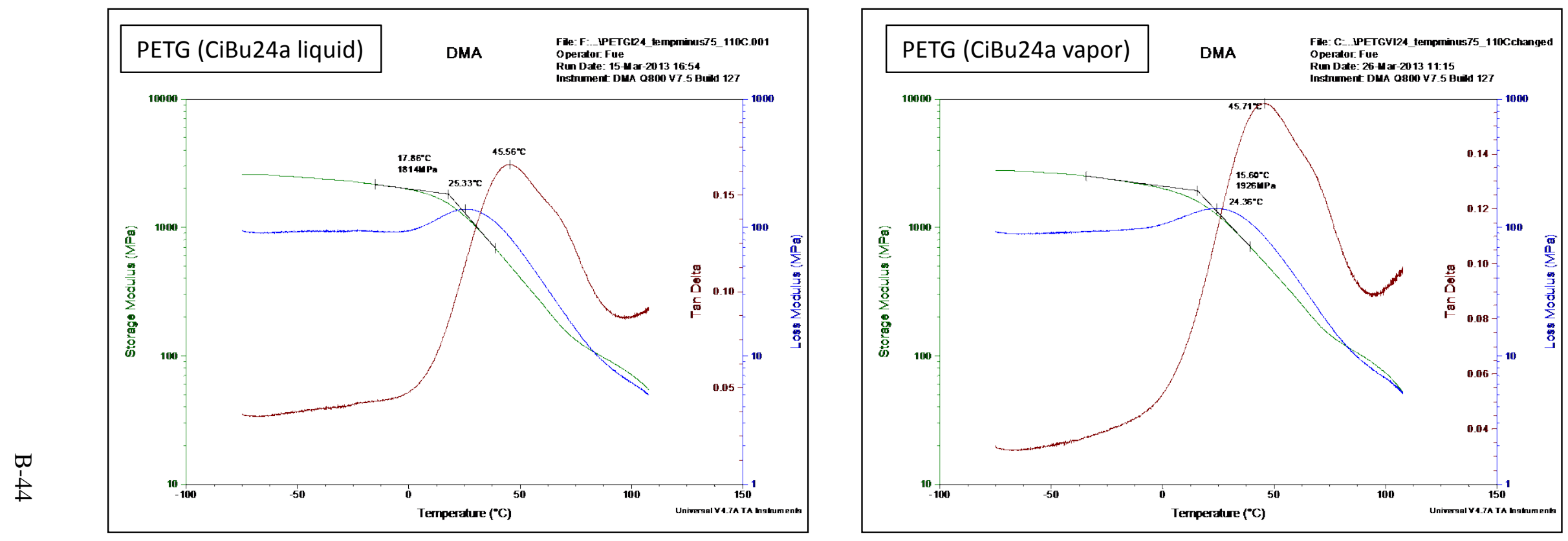
F085 DMA Results for Control, Fuel C, and CE25a Exposures

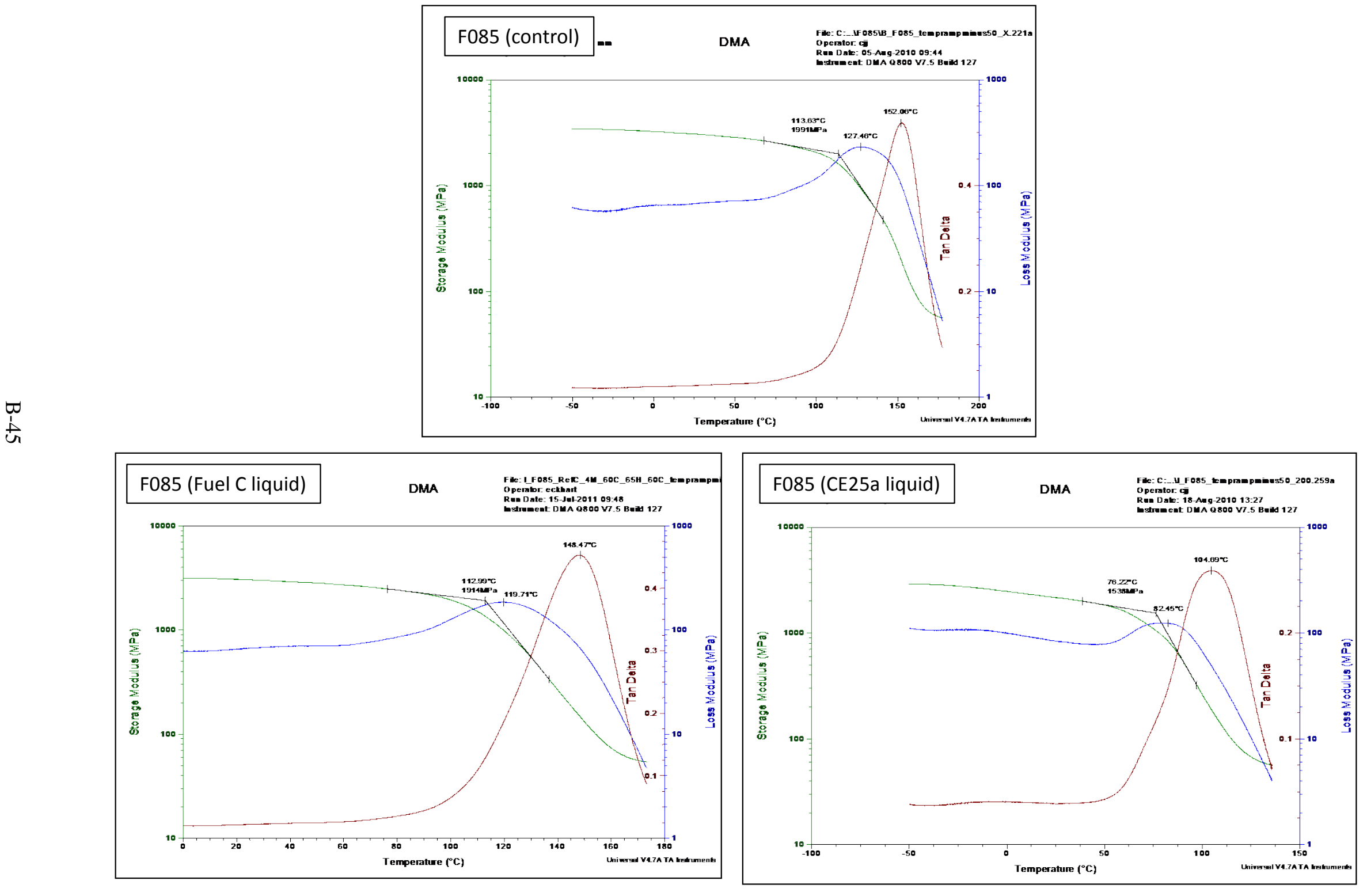


F085 DMA Results for CiBu16a and CiBu24a Exposures
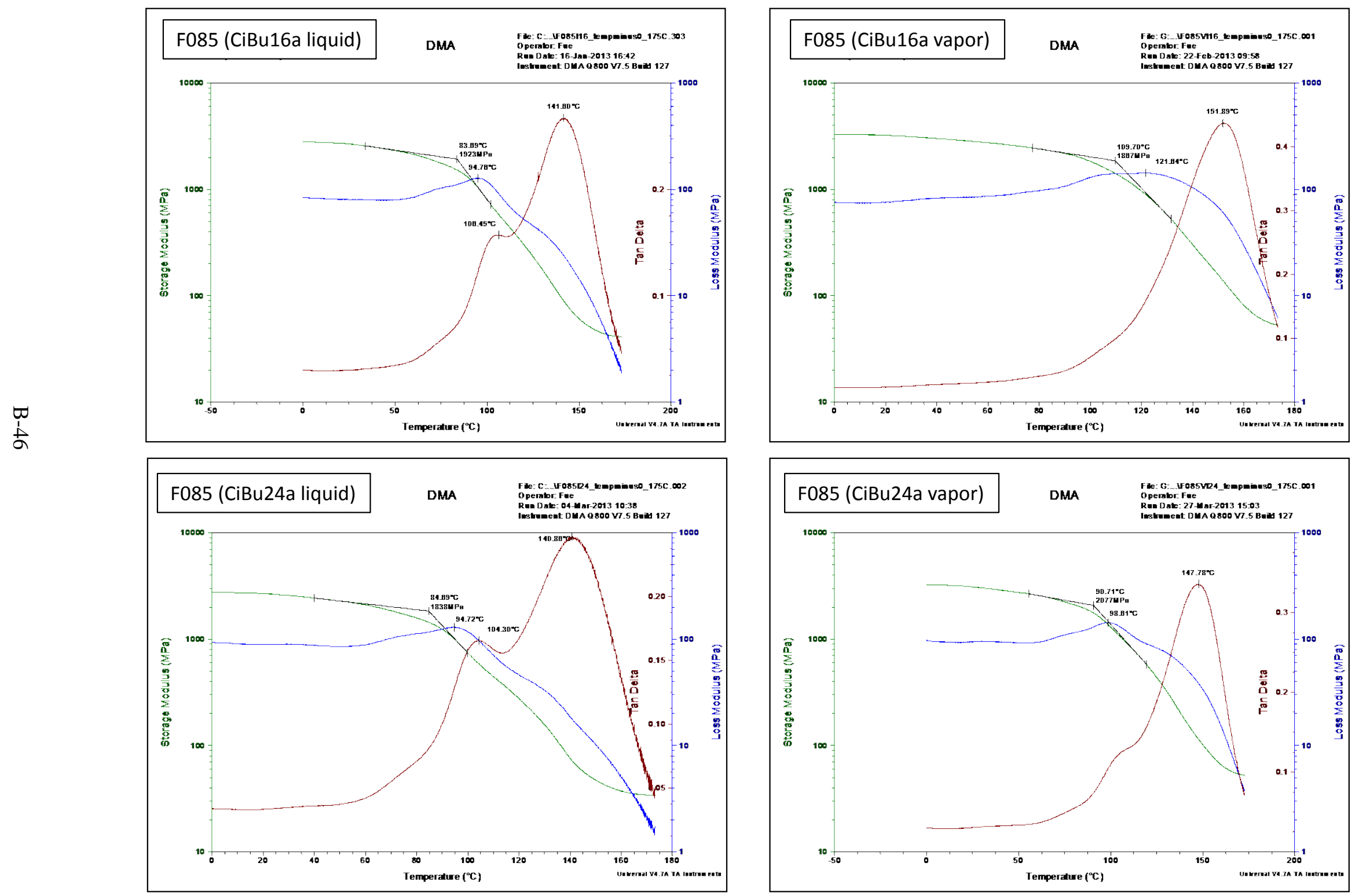
F774 DMA Results for Control and Fuel C Exposures
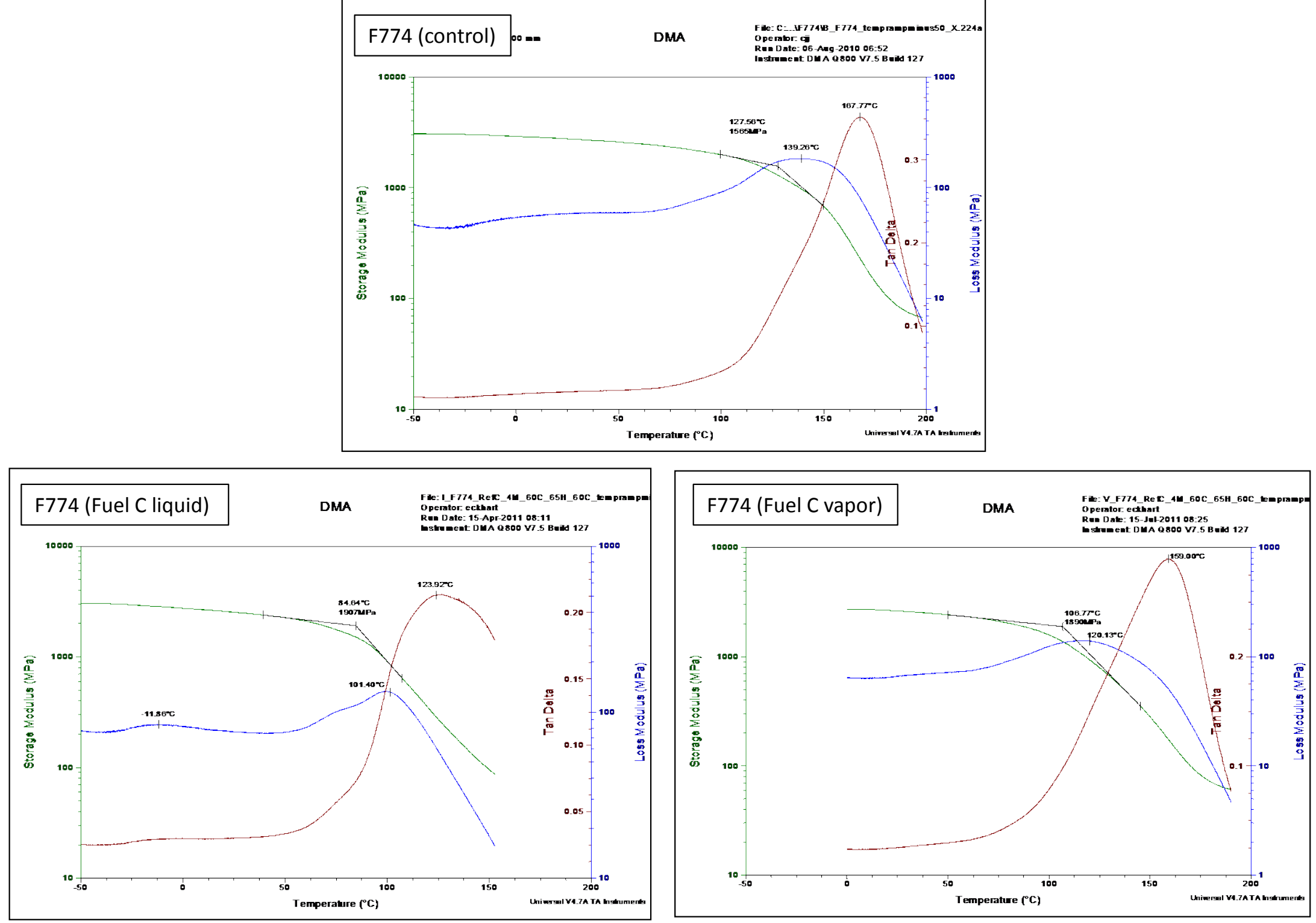
F774 DMA Results for CE25a and CiBu16a Exposures
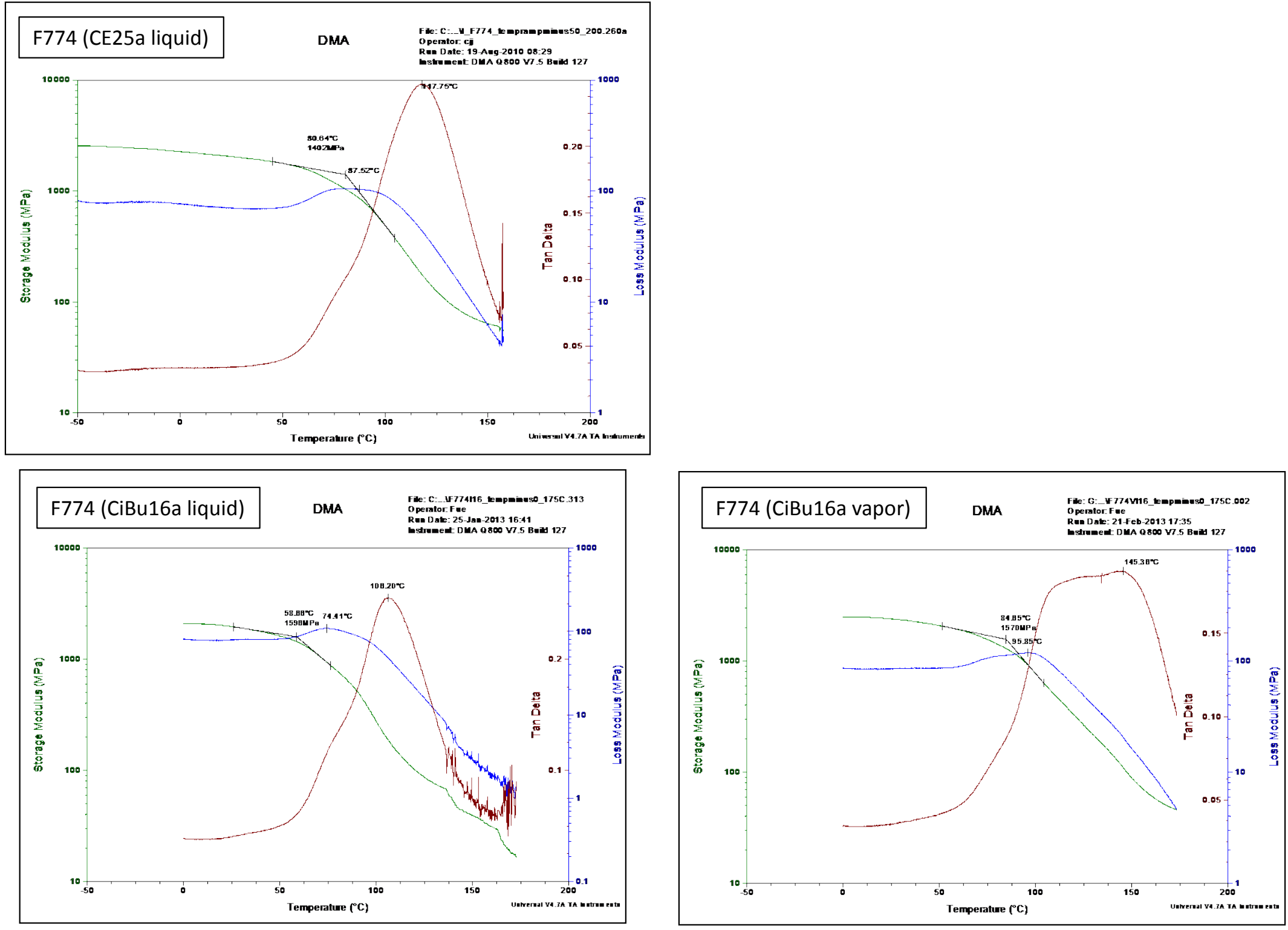


\section{F774 DMA Results for CiBu24 Exposures}
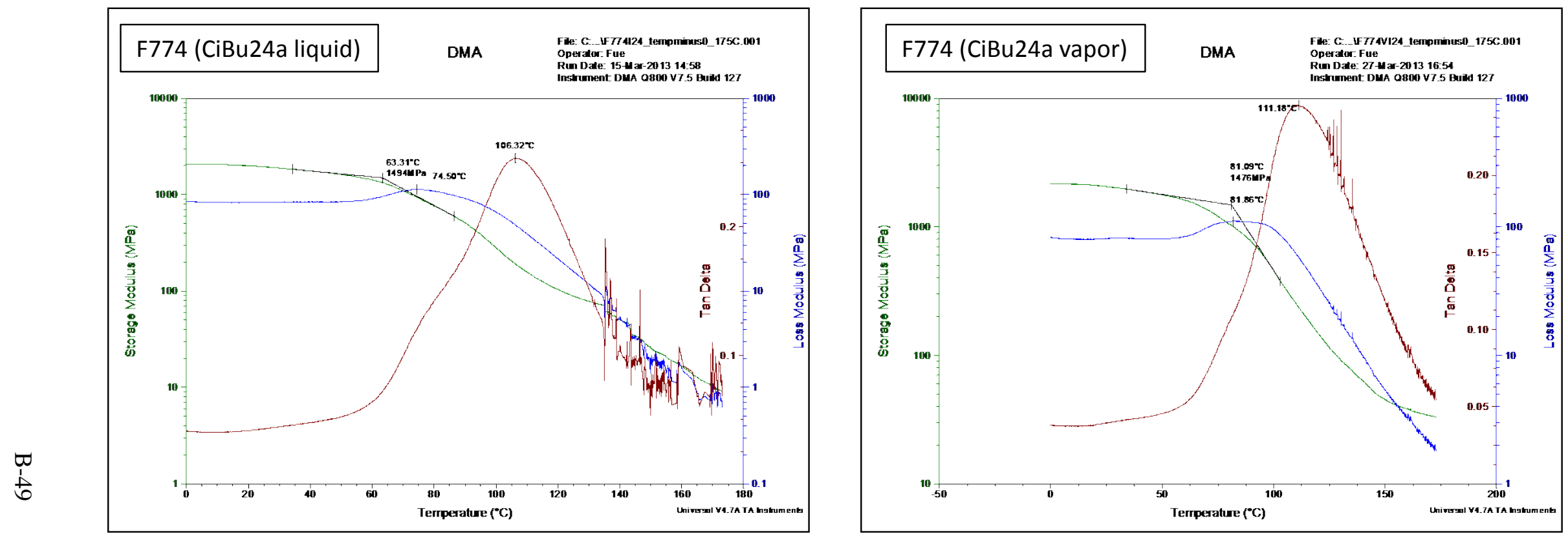
F764 DMA Results for Control and Fuel C Exposures
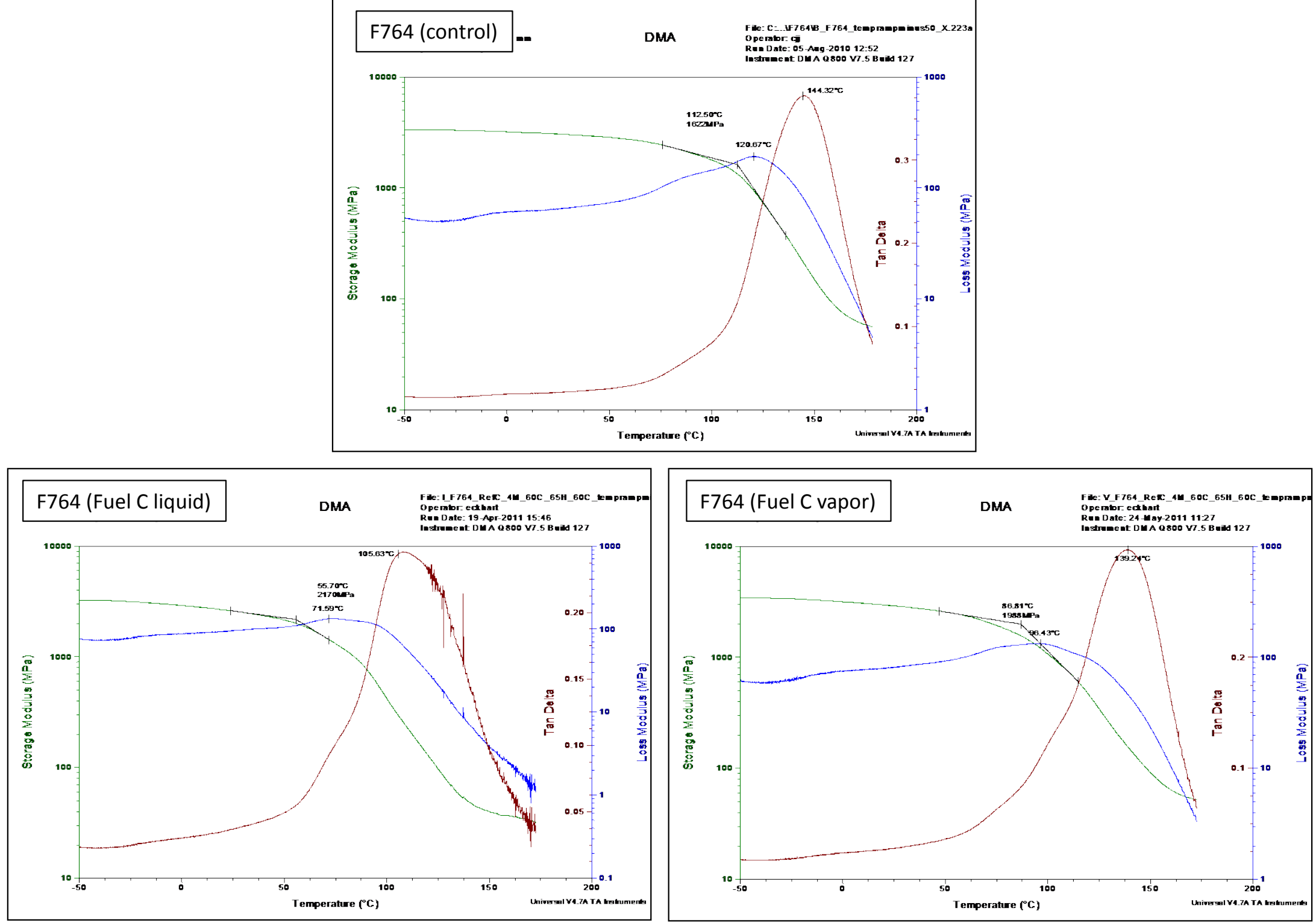
F701 DMA Results for Control and Fuel C Exposures

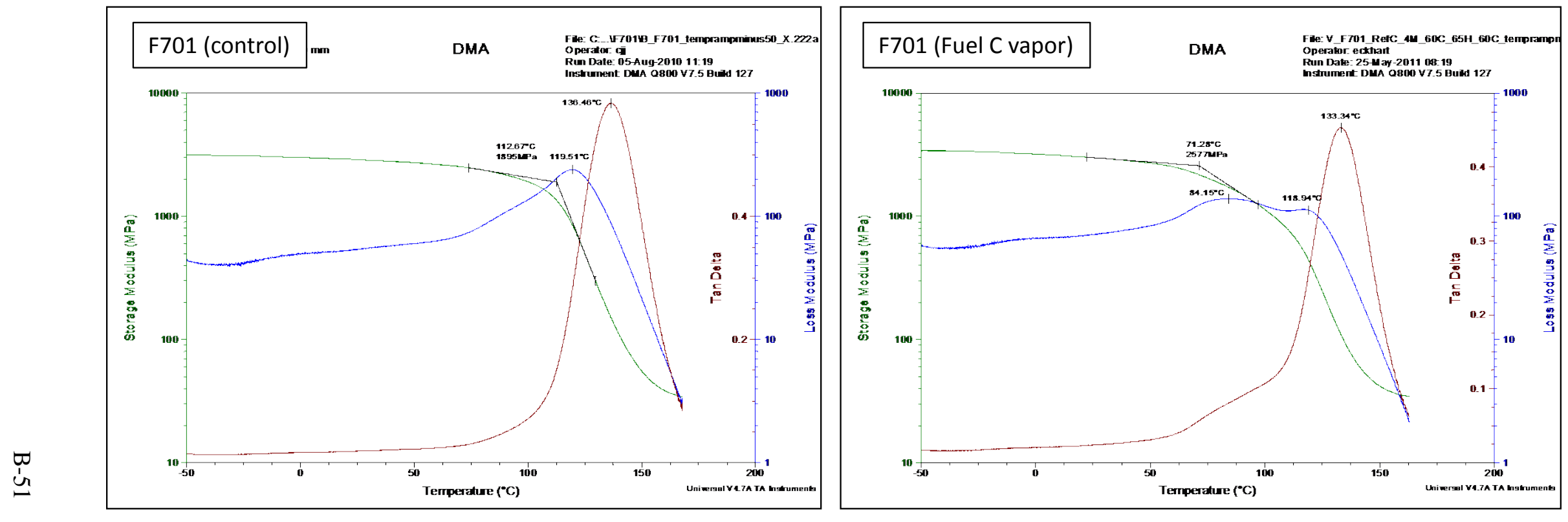




\section{PTU DMA Results for Control and Fuel C Exposures}
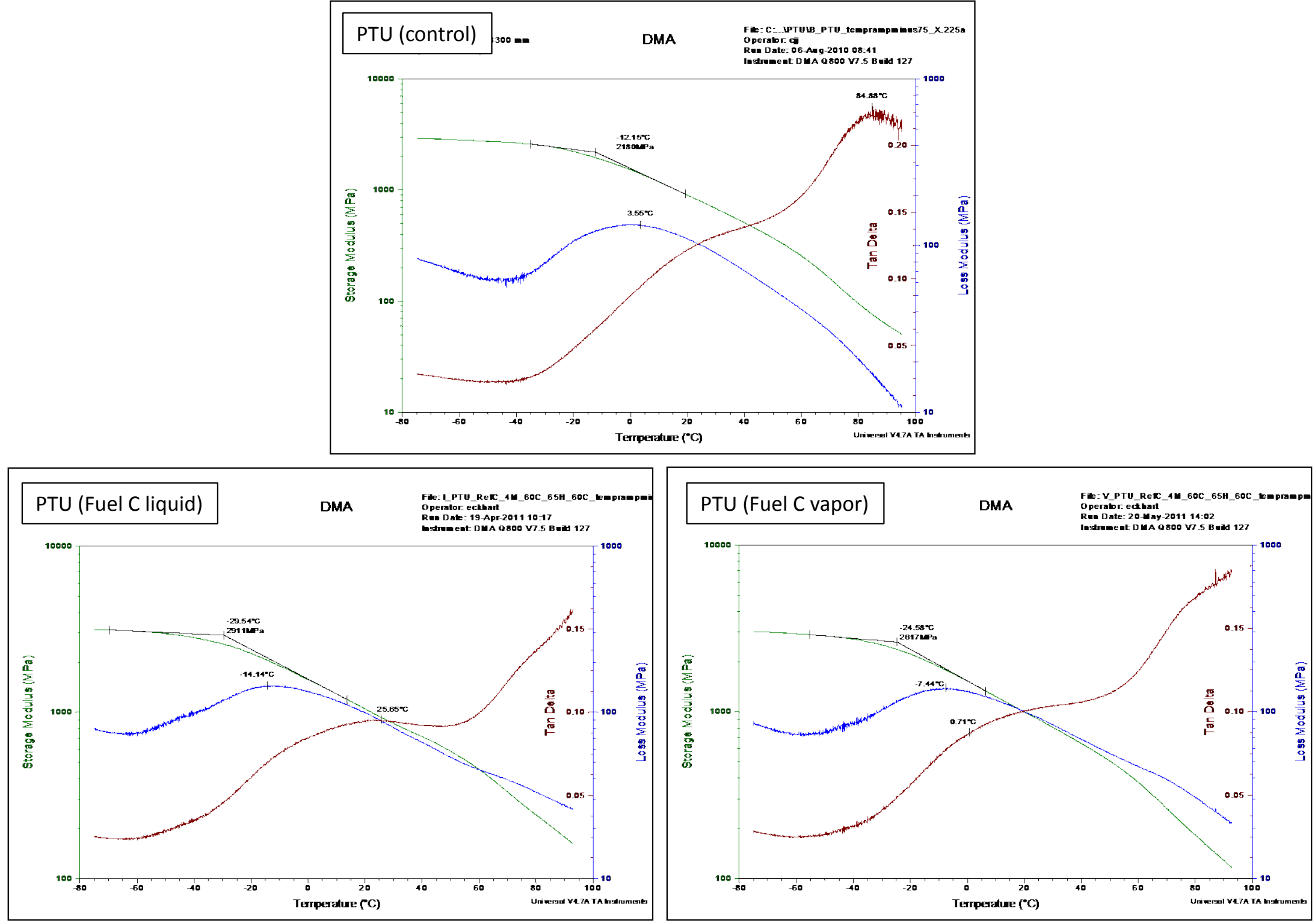


\section{PTU DMA Results for CiBu16a and CiBu24a Exposures}
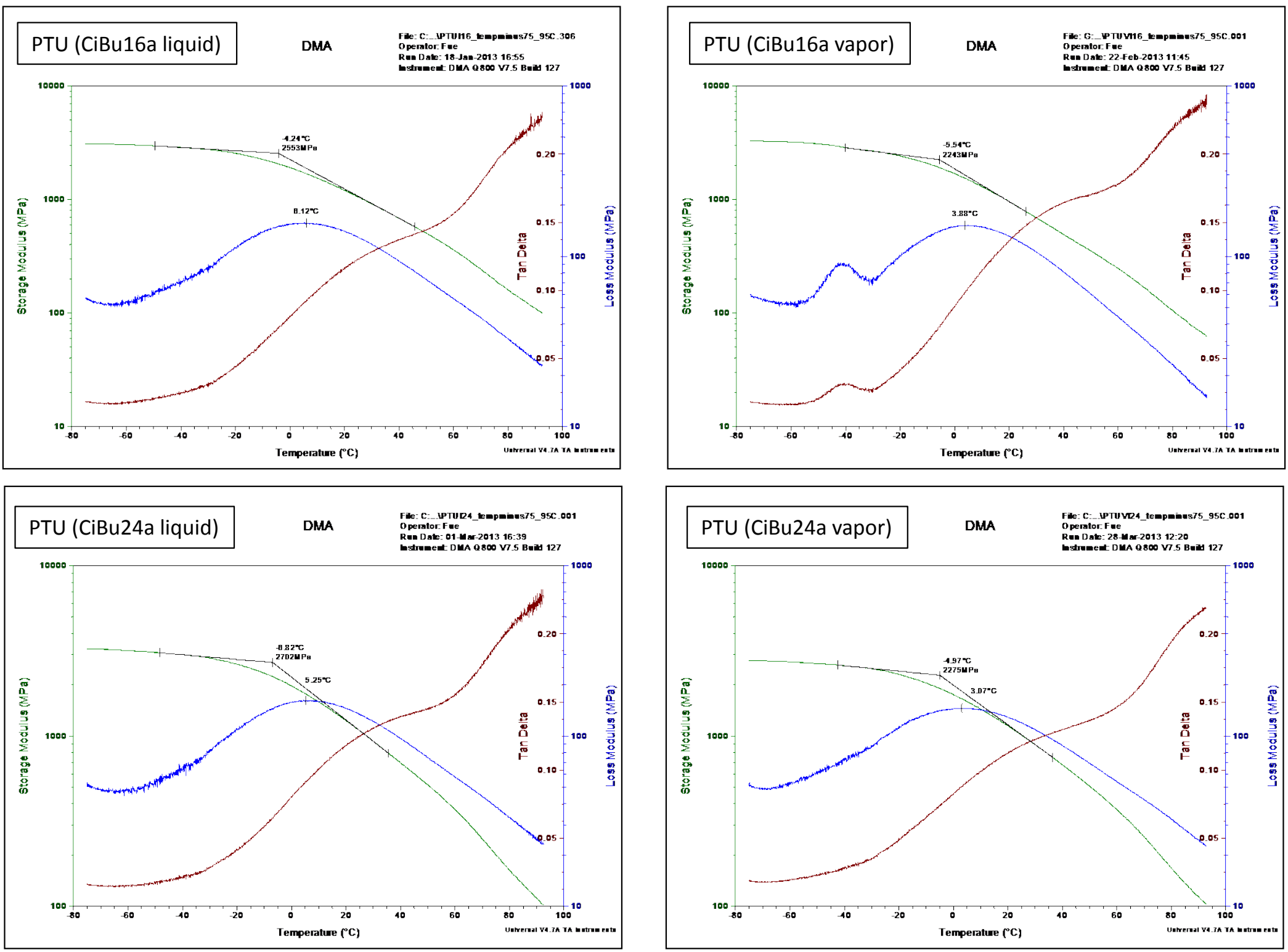


\section{Hot-cured Epoxy DMA Results for Control and Fuel C Exposures}

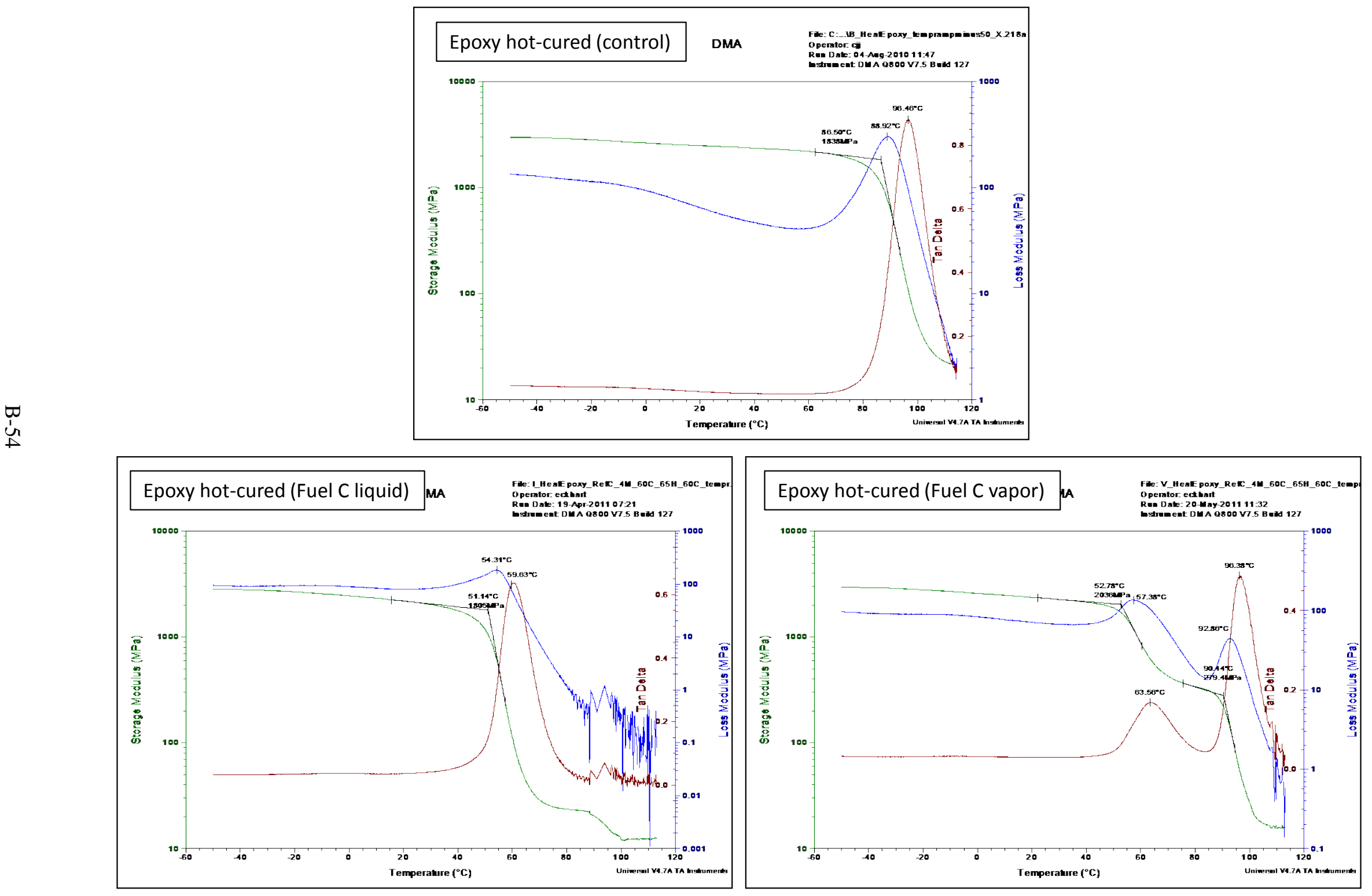


Room Temperature-cured Epoxy DMA Results for Control and Fuel C Exposures

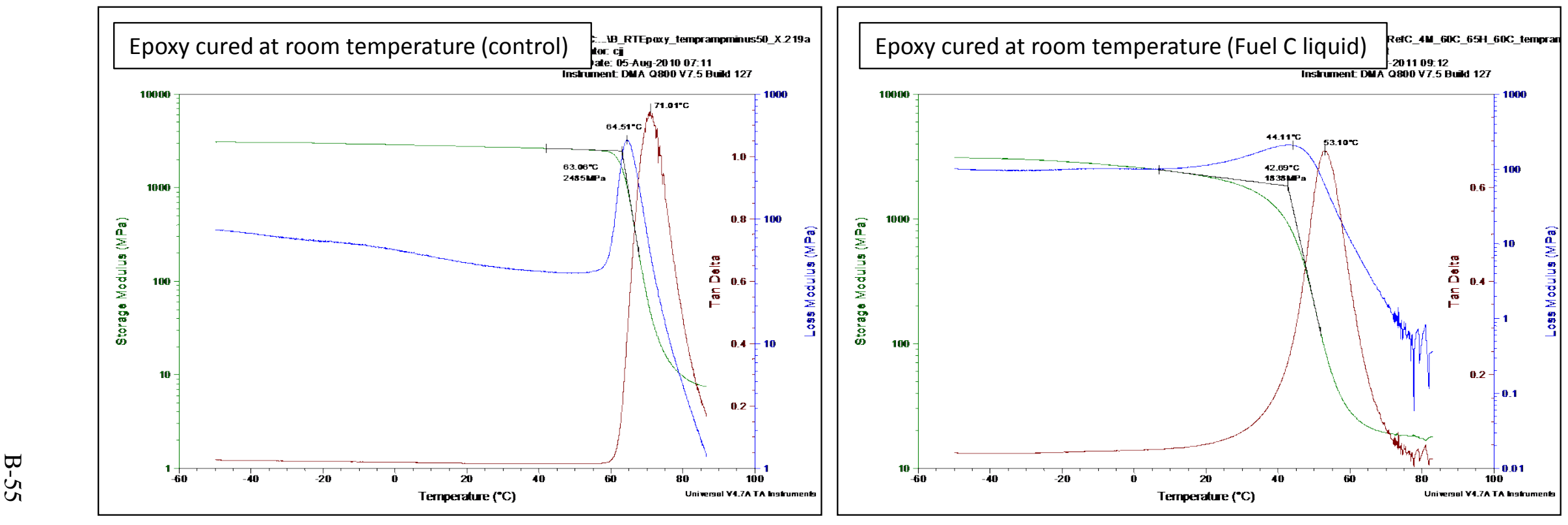




\section{Cork/ECO DMA Results for Control and CiBu16a Exposures}
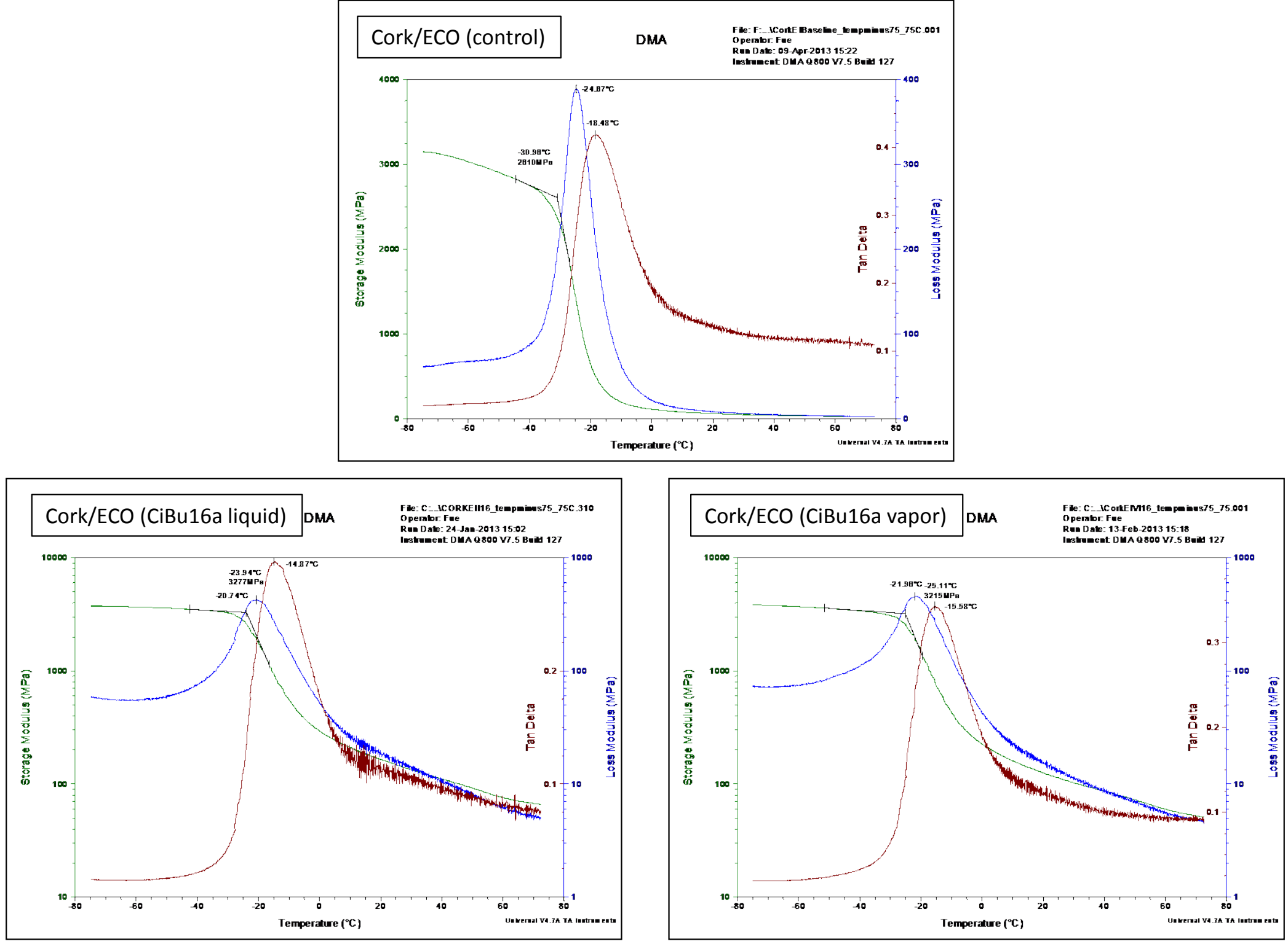
Cork/ECO DMA Results for CiBu24a Exposures
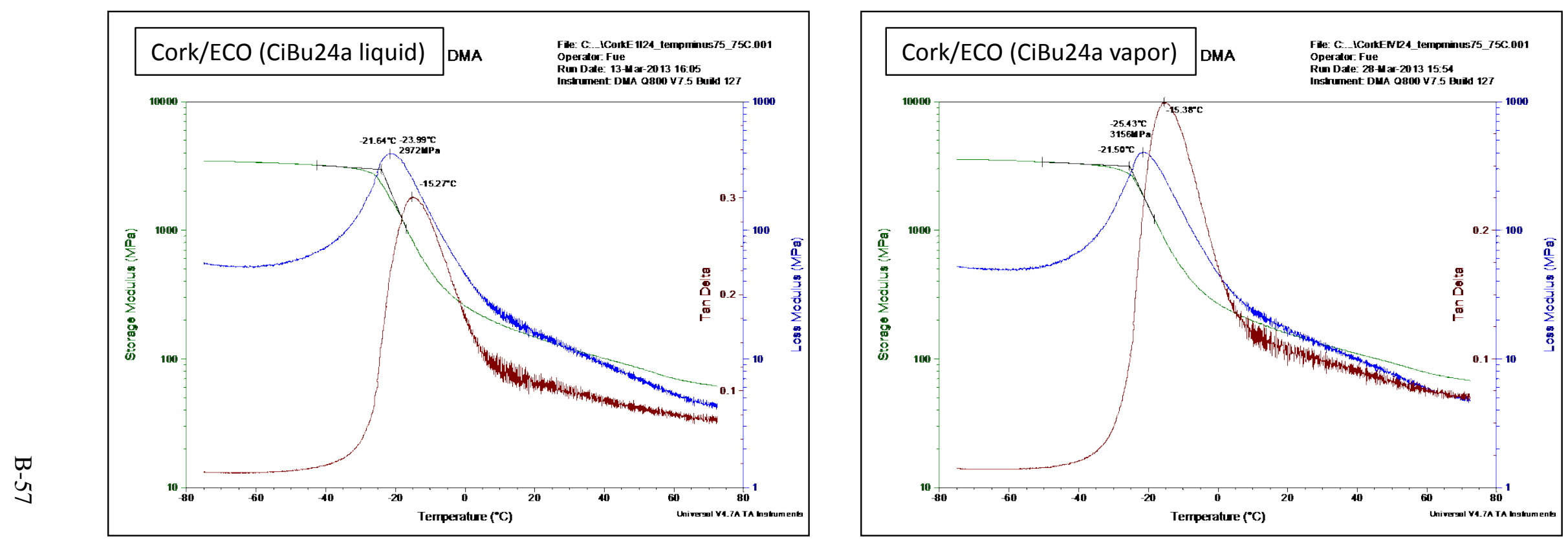


\section{Cork/NBR DMA Results for Control and Fuel C Exposures}
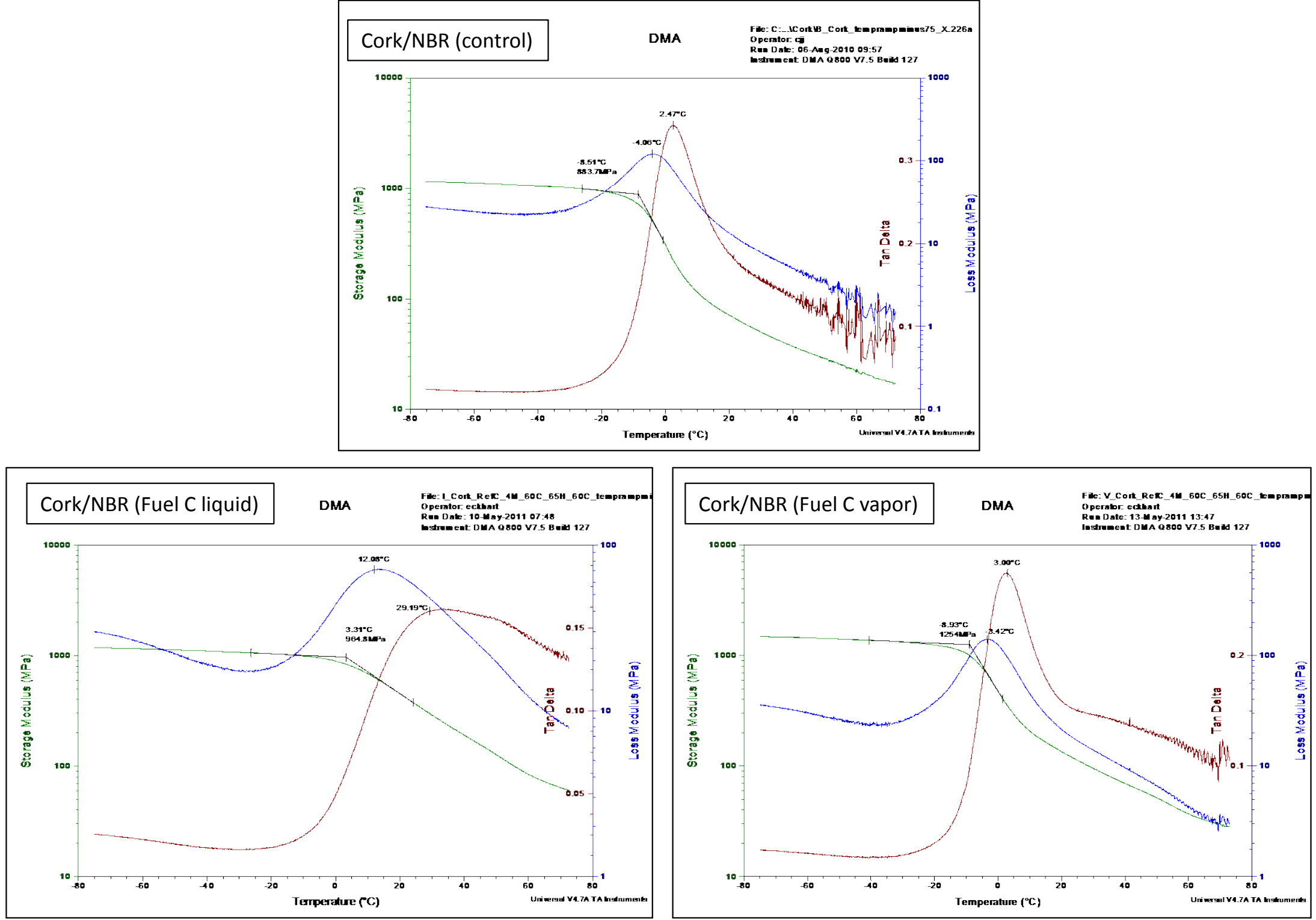


\section{Cork/NBR DMA Results for CE25a and CiBu16a Exposures}
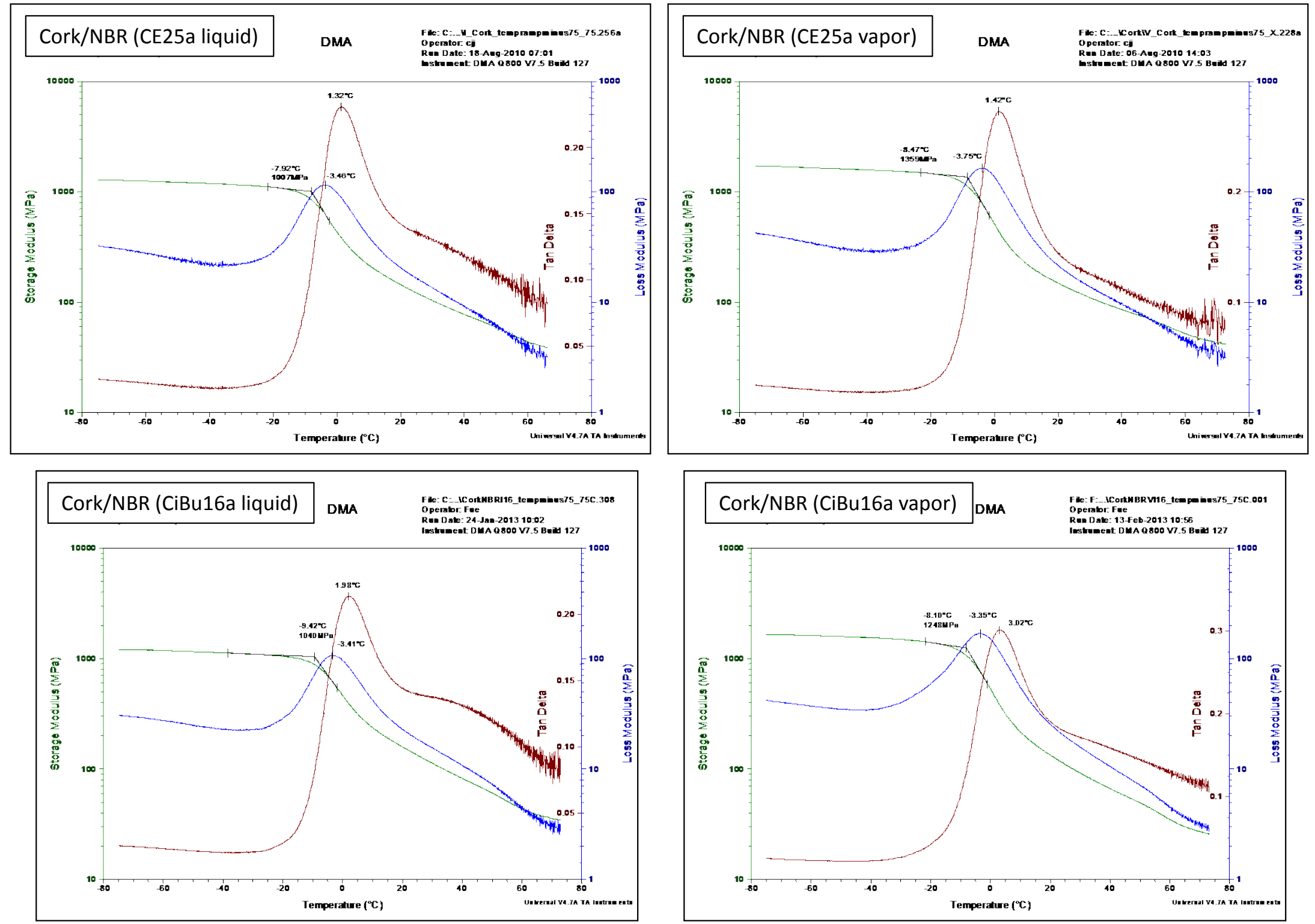
Cork/NBR DMA Results for CiBu24a Exposures
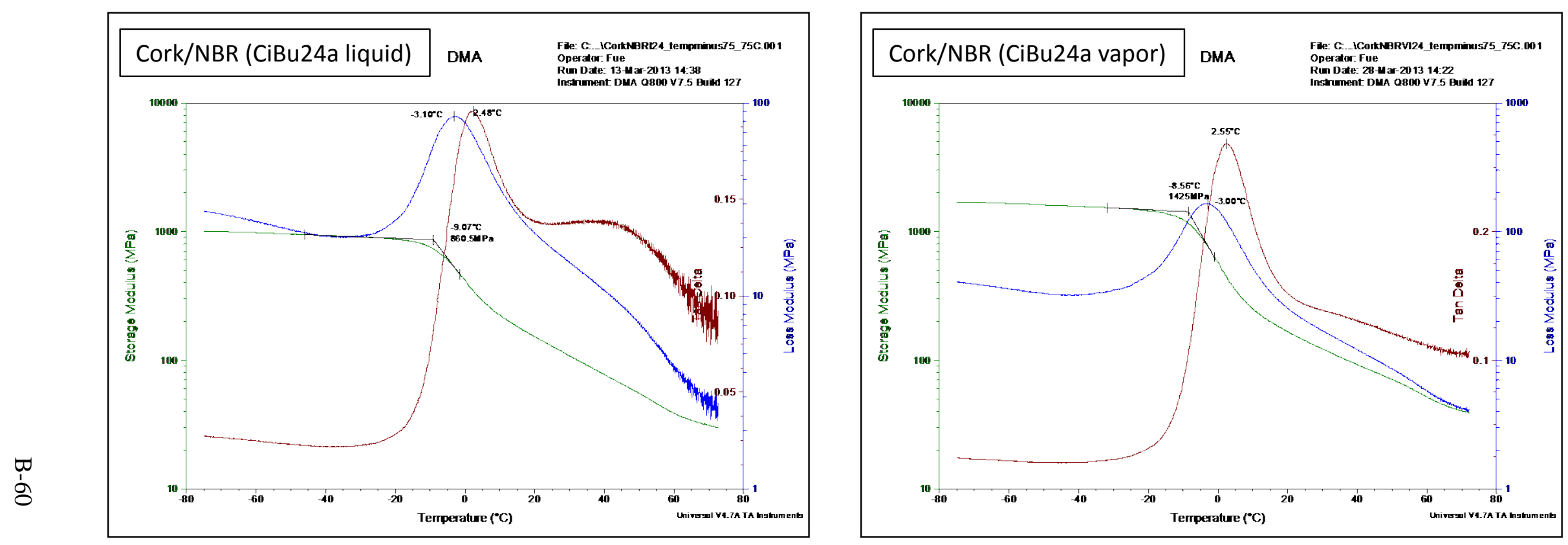